$$
\text { . }
$$




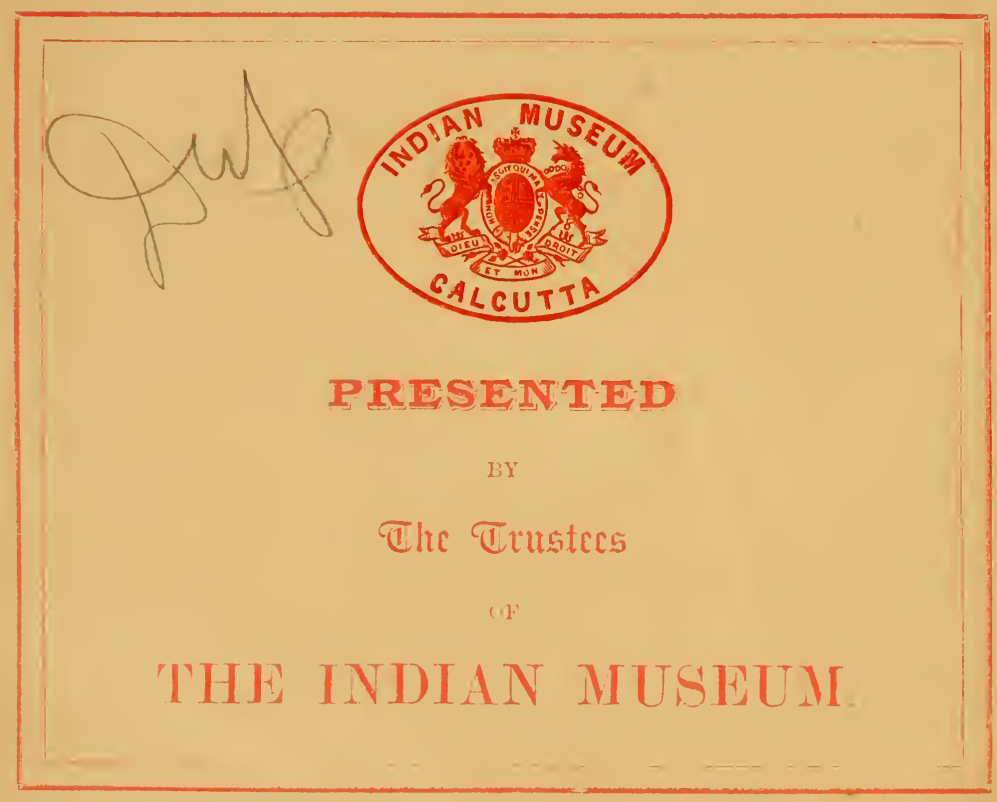





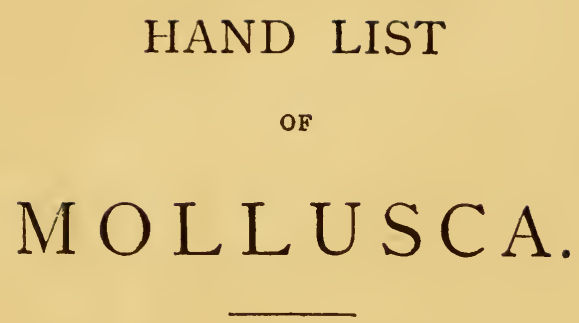

INDIAN MUSEUM. 
CALCUTTA:

OFFICE OF SUPERINTENDENT OF GOVERNMENT PRINTING,

15110 


\title{
HAND LIST
}

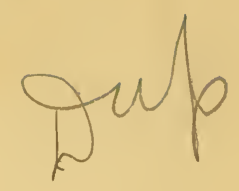

\author{
OF \\ M O L L U S C A
}

IN THE

INDIAN MUSEUM,

CALCUTTA,

BY

GEOFFREY NEVILL, C.M.Z.S.

\author{
PART II. \\ GASTROPODA. \\ Prosobranchia-Neurobranchia (contd.).
}

CALCUTTA :

PRINTED BY ORDER OF THE TRUSTEES.

I 884 . 



\section{PREFACE.}

Since the First Part of the Hand List of Mollusca was published, Mr. Nevill has been compelled by the failing state of his health to resign his appointment as First Assistant in the Indian Museum. Before his departure for Europe, on the 12th March, 1883, he had made over to me the manuscript of the Second Part of the Hand List, which was shortly afterwards placed in the hands of the printer; but as the majority of the proofs had to be sent to Europe to Mr. Nevill for correction, the printing has consequently been much delayed.

It is to be regretted that Mr. Nevill's resignation will prevent any further Parts appearing, until a qualified person has been found to complete the work he has so well begun.

JOHN ANDERSON,

Superintendent.

The 25th January 1885. 



\section{NAMES OF CONTRIBUTORS MENTIONED IN THIS HAND LIST.}

Adpams: Mr. H. A. Adams, England.

ANCEY : Mr. C. F. Ancey, Marseilles.

Anderson: Dr. J. Anderson, F.R.S., Superintendent, Indian Museum.

Armstrong: Dr. J. A rmstrong, Marine Survey of India.

BALL: Mr. Valentine Ball, F.R.S., late of the Geologicul Survey of India, Director of Museum of Science and Art, Dublin.

BAXTER: the late Mr. J. B. Baxter.

Beadue : Reverend E. R. Beadlé, Pliniadelphia.

Beadfort : Miss Beanfort.

Beddome: Colonel R. H. Beddome, late Conservator of Forests, Madras.

Berkelex: Colonel E. S. Berkeley, Port Blair.

BLANFoRd : Mr. H. F. Blanford, F.R.S., Meteorological Reporter, Government of India.

Buanford : Mr. W. T. Blanford, F.R.S., late Geological Surrey of India.

Bouvikr: Mr. P. Bouvier, Marseilles.

BowIe: Major M. M. Bowie, Inspector-General, Police and Jails, Nagpore, Central Provinces.

Brévière: Mr. P. Brévière.

Brown : Mr. W. A. Brown.

Bother: the late Captain Jolın Butler, Political A gent, Naga Hills.

Caldwell: Mr. J. Caldwell, Mauritius.

Chenneli : Mr. A. W. Chenuell, Topographical Survey of India.

Clarie: Mr. G. R. Clarke.

Cockburs: Mr. John Cockburn, Opium Department.

Cox: Dr. J. C. Cox, Sydney.

JodGsoN : Mr. W. Dodgson.

DoHRN : Dr. H. Dolırn, Stettin, Germany.

Dupont : Mr, Evenor Dupont, Mauritius.

FAGOT: Mr. P. Fagot.

FaIRbank : Reverend S. B. Fairbank, Bombay.

FEDden: Mr. F. Fedden, Geological Survey of India.

Folin : Marquis Leopold de Folin, Biarritz.

FORD : Colonel B. Ford, formerly Superintendent of Port Blair and Nicobars. Fritsch : I)r. G. A. Fritsch, Berliu.

Godwin-Austen: Lieutenant-Colonel H. H. Godwin-Austen, F.R.S., late Deputy Superintendent, Topographical Survey of India. 
HACKET : Mr. C. A. Hacket, Geological Survey of India.

Hungerford : Dr, Richard Hungerford, British Medical Service.

Hutron: the late Captain F. W. Hutton, Bengal Staff Corps.

Inrivg: Mr. C. J. Irving, Auditor General, Straits Settlement.

ISSEL: Professor Arturo Issel, Genoa.

Jerdon: the late Dr. T. C. Jerdon.

JickeLI : Dr. C. F. Jickeli, Würzburg, Germany.

Joly : Mr. P. Joly.

Kelaart : the late Dr. E. F. Kelaart, Ceylon.

KING: W. King, D. Sc., Deputy Superintendent, Geological Surrey.

KURZ: the late Mr. S. Kurz, Curator of the Herbarium, Botanical Gardens,

Sibpore.

LAYARD : Mr. E. L. Layard, H.B.M.'s Consul, Noumea.

LAYARD: the late Mr. Frederic Layard.

LEA : Mr. J. Lea.

LEwIS : Captain T. J. Lewis, R.N.R.

LIÉNARD : Mr. Liénard.

LimboRG: Mr. O. Limborg.

MaInwaring: Major-General G. B. Mainwaring, late Bengal Staff Corps.

MaLHerbe : Mr. A. Malherbe, France.

MARTENS : Dr. Eduard von Martens, Berlin Museum.

Mohrenstern : Baron G. Schwartz von Mohrenstern.

Morelet: Mr. A. Morelet, Uijon, France.

Murray: Mr. J. A. Murray, (urator, Karachi Museum.

Nevilu: Mr. A. Nevill, Langham, England.

Nevilu: Mr. G. Nevill, late Indian Museum.

Nevilu : Mr. H. Nevill, Ceylon Civil Service.

NeVILL: Mr. T. F. Nevill.

Newcomb : Professor Dr. W. Newcomb, Cornell University, America.

OLdHAM: the late Mr. T. Oldham, LL.D., F.R.s., Superintendent, Geolozical Survey of India.

Peal : Mr. S. E. Peal, Sibsagar, Assam.

Pease: the late Mr. H. Pease, Honolulu.

Pelly : Miss Pelly.

Power: Surgeon-Major Power.

RABAN : the late Mr. H. Raban, Bengal Civil Service.

RichthоғеN : Professor Baron F. von Richthofen, Berlin.

Roepstorfa : the late Mr. F. A. de Roepstorff, Assistant Superintendent Andaman Islands.

Rogers : the late Major Rogers, of Port Blair.

Schneider: Mr. G. Schneider.

SEMPER : Professor Dr. C. Semper, Würzburg, Germany. 


\section{rii}

Shepard : Professor Shepard.

Shrowill : the late Captain Sherwill.

Sowerby : the late Mr. G. B. Sowerby, London.

Stearns: Professor E. C. Stearns, Washington.

Stoliczka: the late Mr. F. Stoliczka, Ph. D., Palæontologist, Geological Survey of India.

Temple: Captain R. C. Temple, Cantonment Magistrute, Umballa.

Theobald : Mr. W. Theobald, late Geological Survey of India.

Tonnerre: Dr. E. C. Tonnerre, late Health Officer, Calcutta Municipality.

Townsend: Dr. Edmond Townsend, British Medical Service.

Troschel: the late Professor H. Troschel, Bonn, Germany.

WARneford : Reverend J. Warneford, formerly Chaplain, Port Blair, Andaman Islands.

WEBSTER: Mr. E. Webster, Liverpool.

WeinkadfF : Dr. H. C. Weinkauff, Kreuznach, Germany.

Wı̣Llson : Mr. J. Willson, Bengal Educational Department.

WrLMER : Major L. W. Wilmer, Her Majesty's 10th Regiment.

Wrse : Dr. Wise, Dacea.

Wood-MASON : Mr. J. Wood-Mason, Deputy Superintendeut, Indian Museum,

Young: Mr. A. G. Young, Kulu, Kangra. 


\section{LIST OF ABBLLVIATIONS OF NAMES OF AU'THORS.}

A D, $=$ Adams

ANTH. $=$ Anthony.

Bens. or Bs. = Bensun.

BLF. = Blınford.

BoURG. = Bourguignat

Broc. $=$ Brocchi.

BRUG. = Bruguière.

BROM. = Brumati.

CARP. or Cpr. = Cirpenter.

Charp. = Charpentier.

C'Les. = Clessiu.

CR. \& F. $=$ Crosse \& Fischer.

Desh. $=$ Deshayes.

DESMr. = Desinoulins.

$I_{\mathrm{K} R}$ = = Dunker.

DRAP. = Draparnaud.

DYв. = Dybowski.

EYD. \& SouL. = Eydoux and Souleyet.

$\mathrm{FABR} .=$ Fabricius.

FÉr. = Férnssac.

$F_{R}=$ von Frauenfeld.

G.-AUST. = Golwin-A usten.

Gerst. $=$ Gerstfeld.

Gud. = Gould.

Gur. = Gmelin.

GREDL. $=$ Gredler.

GRIFF, $=$ Griffith.

$H_{A L D}=$ Hahlemanu.

HAxL. = Hanley.

$\mathrm{JEF} .=$ Jelfreys.

$\mathrm{L}_{\text {AX. }}=$ Lamarck.

$\mathrm{L}_{\mathrm{AY}} \mathrm{I}_{\mathrm{S}}=$ Layarl.

LESS. = LESSON.

i,LN $=$ Linnæus.
Lor = Lovéll.

MHLF. = Müblfeld.

М ICH. = Michaul.

MONTER. = Monterosito.

Mor. or MorL. $=$ Morelet.

Mous. or Mss. = Mousson.

MüLL. = Müller.

$\mathrm{NEV}_{\mathbf{0}}=$ Nevill.

Newc. $=$ Newcomb.

NiLss. $=$ Nilsson.

ORB. = d'Orbigny.

PALAD. $=$ Paladilhe

PARR. $=$ Parreyss.

PAYR. = Payrandeau.

PFr. $=$ Pfeiffer.

PHIL. = Philippi.

RAF. = Rafinesque.

$\mathrm{RECL}_{\bullet}=$ Récluz.

Rossur. $=$ Rossmässler.

$\mathrm{Rv} .=$ Reeve.

ScHw. $=$ Sthwartz von IIohren . stern.

SDB. $=$ Sandberger.

SHUT. $=$ Shuttleworth .

SovL. $=$ Souleyet .

Sodv. = Souverbie.

Sow. = Sowerby .

STOL. $=$ Stoliczka .

Sw.= Swainson.

T.-CANEFRI = Tapparone-Cane-

fri.

Tнеов. or $\mathrm{TH} .=$ Theobald.

TRIST $=$ Tristram.

Trosch. $=$ Troschel.

$\mathrm{VAL}_{\text {. }}=$ Valenciennes. 
SYSTEIIATIC INDEX.

\section{I.-Sub-Class Gastropoda.}

II.-Order Prosobranchia.

\section{II.-Sub-Order Ctenobratchia.}

\section{I.-Group Taenioglossa.}

Section A. Holostoma-

Family Ampullariidæ

Genus Ampullaria . $\quad \therefore 1$

Sub-Genus Pomus • 8

" Saulea . . 13

Genus Marisa • • . 13

,. Lanistes. . . 13

Family Valvatidæ : $\quad 15$

Genus Valvata • • • 15

Sub-Genus Lyogyrus . • 17

Genus Heterocyclus • • 17

Family Paludinidæ • • 18

Genus Larina • • • · 18

, Paludina : . 18

Sub-Genus Lioplax : $\quad$. 31

„ Tulostoma — 32

Theloma

, Margarya : : 33

, Neothauma . . 33

Sub-Family Benedictiinæ . 34

Genus Benedictia • . 34

Sub-Family Bythiniinæ • 34

Genus Bythinia . • 34

Sub-Genus Fossarulus 42

, Hydrobioides . 42

Genus Stenothyra • • 42

Family Rissoidæ • • 46

Sub-Family Hydrobiinæ . 46

Genus Hydrobia 46

Sub-Genus Bythinella • 49

"Tryonia : 55

Pseudamnicola . 55

Genus Amnicola • • 56

Sub-Genus Pomatopyrgus • 57

Genus Cochliopa • • 58

" Fluminicola . . 58

" Gillia : . 59
PAGR

Genus Somatogyrus • $\quad$. 59

" Lithoglyphus . • 59

Sub-Genus Spekia • $\quad 61$

" Jullienia . . 61

" Lacunopsis . 61

Genus Emmericia . . 61

"Pachydrobia . . 62

" Hemistomia . • 62

"Tricula • . . 62

" Baikalia . . . 62

,, Trachybaikalia . 63

Sub-Family Skeneinæ $\quad . \quad 63$

Genus Skenea • • • 63

Sub-Family Pomatiopsinæ . 63

Genus Pomatiopsis • $\quad 63$

Sub-Family Assimineinæ . 64

Genus Assiminea . 64

Sub-Family Rissoininæ • 73

Genus Rissoina. . . 73

Sub-Genus Iravadia . 97

Isseliella . . 98

Genus Fairbankia • $\quad$ 99

„Folinia . . . 100

", Rissoa . . . 100

"Alvania . . 105

" Fenella . . . 113

"Onoba . . . 119

Sub-Genus Ceratia . 121

, Hyala . . 121

Genus Amphithalmus 123

, Cingula . . . 124

Sub-Genus Putilla • • 126

Genus Setia • • • 127

,Nevillia . . 129

"Eatoniella . . 129

"Crossea . . . 129

" Anabathron . . 130

" Scaliola . . 130

"Nicrostelma : $: 132$

" Corena. . . 132

”Rissopsis . . 132

Family Heterophrossnidæ • 133

Genus Jeffreysia • • 133

"Barleeia . . . 134 


\begin{tabular}{|c|c|c|c|c|c|}
\hline & & $P_{\triangle G E}$ & & & $P_{\triangle G B}$ \\
\hline Family Littorinidæ & - & . 135 & Sub-Genus Meseschiz & & 202 \\
\hline Genus Littorina & . & . 135 & Genus Anculosa & & - 202 \\
\hline Sub-Genus Tectus & • & - $\quad 155$ & Sub-Genus Eurycaelo & & - 205 \\
\hline ", Echinella & - & . 157 & Genus Lithasia. & & 205 \\
\hline Genus Limnotrochus & - & - 157 & Sub-Genus Angitrem & & 205 \\
\hline " Plesiotrochus & - & - 157 & Sub-Family Melaniin & & - 206 \\
\hline Sub-Family Fossarina & & - 158 & Genus Melanopsis & & 206 \\
\hline Genus Risella : & - & . 158 & Sub-Genus Pseudhem & aisinus & 214 \\
\hline Sub-Genus Peasiella & - & - 159 & Genus Hemisinus & $\cdot$ & 215 \\
\hline Genus Phaneta. & - & 162 & " Doryssa . & - & 216 \\
\hline "Fossar i . & & 162 & " Faunus. & & 217 \\
\hline Sub-Genus Couthouyi & & - 166 & Sub-Genus Melanatri & & 218 \\
\hline Genus Conradia. & - & - 168 & Genus Pirenopsis & . & 218 \\
\hline Sub-Genus Gottoina & - & - 170 & „ Claviger . & - & 219 \\
\hline Genus Raulinia . & - & - 170 & Lartetia . & - & 219 \\
\hline " Cithna . & - & . 170 & Oncomelania & - & 219 \\
\hline Isapis . & - & - 171 & Tiphobia & - & 219 \\
\hline Fossarina & - & - 171 & Pyrgula • & - & 220 \\
\hline Sub-Family Lacuninæ & & - 172 & Moitessieria & - & 220 \\
\hline Genus Cremnoconchus & & - 172 & Lhotellieria & - & $2 \div 0$ \\
\hline " Stenotis . & - & 173 & Paladilhia & - & 220 \\
\hline Lacuna • & - & 173 & Bugesia • & - & 221 \\
\hline "Modulus & - & 175 & "Melania & - & 221 \\
\hline Sub-Family Litiopinæ & & 177 & Duo-cenus Nolangid & & \\
\hline Genus Litiopa. & & 177 & Sulcos & & 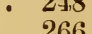 \\
\hline Styliferina & • & 178 & Nigritella & & \\
\hline Diala . & . & 179 & Pachychil & & \\
\hline Alaba & . & . 181 & Acrostom & & $\begin{array}{l}208 \\
270\end{array}$ \\
\hline Planaxis. & 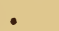 & - 184 & Melanella & & 271 \\
\hline Quoyia • & $\bullet$ & 193 & Paramela & nia & 272 \\
\hline Holcostoma & & 193 & Tarebia & . & 272 \\
\hline Family Melaniidæ & & . 193 & Tiara & - & 278 \\
\hline Sub-Family Ceriphasi & inæ & . 193 & Plotia & . & 280 \\
\hline Genus Io & & - 194 & , . Mainwari & ngia & 286 \\
\hline " Pleurocera & & - 194 & Sub-Family Paludom & inæ & - 287 \\
\hline Sub-Genus Strephobas & isis & - 196 & Geuus Paludom'ss & & - 297 \\
\hline Genus Goniobasis & $\cdot$ & - 196 & Sub-Genus Philopota & mis & - 297 \\
\hline Sub-Genus Schizostom & & - 202 & , Tanalia & - & - 301 \\
\hline
\end{tabular}




\title{
MOLLUSCA.
}

\section{ORder PROSOBRANCHIA.}

\section{Sub-Order CTENOBRANCHIA, Schweigger.}

\author{
[Pectinobranchia, Cuvier].
}

\section{Group TAENIOGLOSSA, Gray.}

Section A.-HOLOSTOMA, Blainville.

\section{Family AMPULLARIID球, Guilding.}

\section{Genus AMPULLARIA, Lamarck.} Linn.

Prod. 1799 and Syst. anim. sans vert. 1801, type Helix ampullacea,

\section{Ampullaria globosa.}

Ampullaria globosa, Swainson, Zool. Ill. 1822, "Indian Rivers"; Ampullaria orbata, Mörch, [as "of Perry 1811"]; from Bangkok, fide Morelet; fide Martens, Mal. Bl. 1857: alt. 74, diam. 78 mil.

3 Jamálpur and Gopálpur; coll. Dr. F. Stoliczka.

50 Calcutta, Pt. Canning, Rániganj and Dacca; coll. Dr. F. Stoliczka, J. Willson and G. Nevill, Esqrs.

4 Allahabad; coll. Major L. W. Wilmer and J. Cockburn, Esq.

7 Sambalpur, Central Provinces; coll. Colonel M. M. Bowie.

5 Máubhum; coll. V. Ball, Esq.

11 Dum-Dum; coll. Major L. W. Wilmer.

1 Kusiára River, above Panchuganj, Sylhet; coll. J. Wood-Mason, Esq.

This last specimen is very interesting, as it was found immediately associated with $A$. maura! Calcutta pecimens usually 
have a yellow peristome, sometimes of deep orange or crimson colour, and occasionally of a pure white!

subvar. sinistrorsa, nov.

Cat. Moll. Fasc. E, July 1877, p. 2, Calcutta.

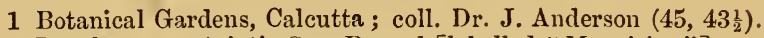

1 Loc.? ; ex c. Asiatic Soc. Bengal [labelled "Mauritins"].

var. minor.

Ampullaria globosa, var. minor, Nevill, Cat. Moll. Fasc. E, 1877, DumDum : long. $36, d .30 \frac{1}{2}$ mil.

8 Dum-Dum, nr. Calcutta (type var.); coll. Major L. W. Wilmer. 2 Siliguri ; coll. Colonel G. B. Mainwaring.

var. incrassatula.

Ampullaria globosa, var. incrassatula, Nevill, Cat. Moll. Fasc. E, 1877,

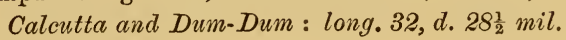

10 Calcutta (type var.); coll. G. Nevill, Esq.

4. Dum-Dum; coll. Major L. W. Wilmer.

var. corrugata.

Ampullaria corrugata, Swainson, Zool. Ill. 1822, "India;" fide Hanley, Syst. List, Con. Indica ? ; = A. hepataria, $R v$.

subvar. longispira.

Long. 68, diam. 63 mil.; rugosely malleated, perist. of a pale yellow colour.

1 Benares; coll. Colonel G. B. Mainwaring.

subvar.

Con. Indica, pl. 113, fig. 2, Loc. ?.

3 Loc. ?; ex c. Asiatic Soc. Bengal and Dr. F. Stoliczka.

subvar.

Ampullaria globosa, var. Reeve, Icon. 1856, fig. 46.

4 Assam [?]; ex c. Dr. T. Oldham.

Smooth and polished, with light yellow coloured peristome. Long. 58, diam. 55 mil. 
subspecies carinata.

Ampullaria cariuata, Swainson, Zool. Ill. II, 1831, "India" ; var. $=A$. paludinoides, Conch._Cab. II, 1851, Mangalore [not of Crist. and Jan].

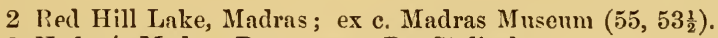

2 Kadapá, Madras Pres.; ex c. Dr. Stoliczka.

8 Madras; coll. G. Nevill, Esq.

5 Calcutta ; coll. G. Nevill, Esq.

1 Loc.? ; ex c. Asiatic Soc., Bengal.

subvar. sinistrorsa, nov.

1 Madras; coll. G. Nevill, Esq. (38, 32).

\section{var. malabarica.}

Ampullaria malabarica, Philippi, Zeits. Mal. 184S, Madras.

7 Madras and Cuddalore; coll. Dr. F. Stoliczka and Colonel Beddome.

These specimens resemble Con. Indica, Pl. 114, fig. 2 [" $A$. malabarica"]!

\section{var. layardi.}

Ampullaria Layardi, Reeve, Icon. 1856, Ceylon.

30 Ceylon; coll. G. Nevill, Esq.

4 Ceylon; coll. E. L. Layard, Esq. [A. S. B.].

2 Loc. ?; ex c. Asiatic Soc., Bengal. ardi"'].

Well represented by Con. Indica, Pl. 114, fig. $4[" A$. laysubvar. virens.

? Ampullaria virens, Lamarck, Hist. VI, 1822, Hab.?

1 Kollam; coll. Colonel R. H. Beddome.

Most closely resembling the preceding Ceylon form; carination at suture obsolete. Near Kiister's Pl. 8, fig. $2[$ [ $A$. virens"].

\section{Ampullaria nux.}

Ampullaria nux, Reeve, Icon. 1856, "Bombay."

8 Khandála, Bhore Ghât, Bombay; coll. W. I Hlauford, Esq., and Rev. S. B. Fairbank. 
var. (? n. sp.)

9 Trauquebar (?); coll. Capt. Lewis (A. S. B.).

\section{A mpullaria maura.}

Ampullaria maura, Reeve, Icon. 1856, Loc. ?.

30 Tezpur, \&c. (Assam); ex c. Dr. F. Stoliczka.

30 Sibságar and Gauhátí, Assam; coll. S. E. Peal, Esq., and Museum Collector.

2 Darrang and Narainpur, Assam; coll. Colonel H. H. Godwin-Austen.

Long. 53, diam. 49 mil.

30 Silchar and Silcuri, Cachar; coll. J. Wood-Mason, Esq.

5 Kusiára River above Panchuganj, Sylhet; coll. J. Wood-Mason, Esq.

var. theobaldi.

Ampullaria Theobaldi, Hanley, Con. Indica, 1875, pl. 115, Loc.?, and P. Z. S. 1875 , Bhamó.

12 Bhamô ; coll. Dr. J. Anderson (long. 52, diam. 53 $\frac{1}{2}$ ).

Apparently none of them quite adult!

\section{Ampullaria aperta.}

Ampullaria aperta, Philippi, Zeits. Mal. 1849, Loc.?; spec. juv.; Con. Indica, pl. 115, fiy. 4, as "A. saxea, var., Pegu."

3 Pegu; ex c. Dr. F. Stoliczka.

6 Akyab; coll. Dr. F. Stoliczka.

2 Poungday, Prome Distr.; coll. Colonel G. B. Mainwaring.

These specimens agree with Con. Indica, Pl. 115, fig." 4.

var. saxea.

Ampullaria saxea, Reeve, Icon. 1856, fig. 108, Loc ?; Con. Inctica, pl. 118, fig. 3, as "A. saxea."

1 Bassein; coll. W. Theobald, Esq.

var.

? Ampullaria olea, Reeve, Icon., fig. 102, Hab. ?; Con. Indica, System. List, 1876.

3 Cachar (juv.); ex c. Dr. F. Stoliczka. 


\section{Ampullaria conica.}

Ampullaria conica, Gray, Wood's Index Test. Sup. 1828, and Hanley, Conch. Misc., pl. III, fig. 13; = A. scutata, Mousson, Moll. Java, 1849 [not of Phil.]; fide Martens, var. = A. javanica, Rv. 1856 ; from Cambodia fide Morelet.

10 Moulmain ; coll. Baron F. von Richthofen and Dr. F. Stoliczka. 11 Akyab; coll. Dr. F. Stoliczka.

\section{var. expansa.}

Ampullaria conica, var. expansa, G. Nevill, Cat. Moll. Fasc. E. 187\%, Pegu; $=$ A. paludinoides, Con. Indica, pl. 114, fig. 5 [not of Philippi or Crist. and Jan].

7 Pegu (type var.); coll. W. Theobald, Esq.

3 Tenasserim (?); ex c. Dr. F. Stoliczka.

1 Mandalay (?); ex c. Dr. F. Stoliczka.

var. compacta.

Ampullaria compacta, Reeve, Icon. 1856, figs. 62 and 71, Malacca.

5 Tenasserim Prov.; coll. Dr. F. Stoliczka and Mr. Ossian Limborg.

8 Mandalay; coll. Dr. J. Anderson.

var. orientalis.

Ampullaria orientalis, Philippi, Zeits. Mal.1848, "China?" ; = A. scutata, Phil., Conch._Cab. II, pl. I, figs. 4-5 [not of Mousson].

4. China; ex c. A. Morelet, Esq. [as "A. paludinoides"].

1 Canton; coll. Baron F. von Richthofen.

3 Bassein, Arakan; coll. W. T. Blanford, Esq.

1 Loc. ?; ex c. Dr. F. Stoliczka.

var. borneensis.

Ampullaria borneensis, Philippi, Conch.-Cab. II, 1851, Borneo; fide Morelet, A. borneensis from Bangkok : alt. 45 to 63, diam. 37 to 53 mil.

2 Singapore; coll. C. J. Irving, Esq.

3 Borneo Straits; ex c. I)r. F. Stoliczka.

\section{Ampullaria ampullacea.}

Helix ampullacea, Linnaeus, Mus. Ulrica, 1764; fide Martens, $=$ A. celebensis, Quoy, 1832; = A. sumatrensis, Phil., Conch.-Cab. II, 1851; = A. magnifica, Dkr., Conch.-Cab. II, Java. 
var. javensis, nov.

Ampullaria celebensis, Mousson, Moll. Java, pl. I, fig. 1, alt. S0, diam. 72 mil., and Conch.-Cab. II, pl. XIX, fig. 3, Java, [not of Quoy].

1 Java; coll. Baron F, von Richthofen.

'This Javanese form differs from Quoy's species by the less produced spire, the more everted aperture, and the more distant and irregular spiral black lines, \&c.-Alt. 63, diam. 56 mil., agreeing exactly with the above-quoted figure in Conch.-Cab. II.

\section{Ampullaria turbinis.}

Ampullaria turbinis, Lea, Journ. Acad. Philad. VI, 1856, Siam and Obs. XI, pl. 22, fig. 2; from Battambang in Cambodia: alt. 80 , diam. 77 mil., fide Morelet, Ser. Con. Il.

1 Cambodia (spec. juv.); ex c. $\Lambda$. Morelet, Esq.

Lea describes his species as "scarcely perforate"; his figure appears quite imperforate. Morelet, l. c., deseribes Cambodian specimens as having a large and deep umbilical perforation. He further notices two distinet forms, - one without bands, the other banded, with the bands ceasing abruptly a short distance before the peristome; the former seems to agree with my var. subglobosa, the latter with my var. subampullacea.

var. subglobosa, nov.

4. Java (?); coll, Baron F. von Richthofen.

Alt. $70 \frac{1}{2}$, diam. $69 \frac{1}{2}$ mil. A form with uniform green epidermis, not banded, with white peristome and remarkably depressed and flattened spire, unlike any figure I can find. Possibly from Siam, and if so, probably the form called A. celebensis by von Martens, and $A$. turbinis by Morelet.

\section{var. subampullacea, nov.}

2 Perak; coll. Ed. 'Townsend, Esq.

Alt. 75, diam. 68 mil. Fairly represented by Pl. 19, fig. 1, Conch.-Cab. II, "A. sumatrensis, Phil.", but more "turbinate" - that is, the borly whorl more angularly swollen aboveconsequently, not so convexly rounded; the umbilicus, coloured peristome, and spiral purple bands exactly resemble those of the figure. I gave a third specimen of this form to Monsieur II. Crosse. 


\section{Ampullaria gracilis.}

Ampullaria gracilis, Lea, Journ. Acad. Philad. VI, 1856, Siam; also from Cambodia, J. de C. 18\%\%.

1 Siam; ex c. A. Morelet, Esq.

\section{Ampullaria stoliczkana.}

Ampullaria Stoliczkana, C. Nevill, Cat. Moll. Fase. E, 187\%, Penang, alt. 51, d. 41 mil., and Journ. As. Soc. Bengal, 1881, pl. VI, fig. 11.

6 Penang (type); coll. Dr. F. Stoliczka.

Two are full-grown, and four young.

\section{Ampullaria polita.}

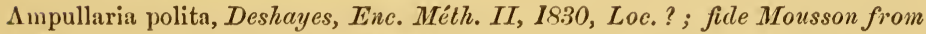
Java; fide Martens from Manila and? = A. lubrica, Rv. : fide Morelel, all. 95, diam. 66-another alt. 55, d. 42 mil., and also monstrosily $=A$. pagoda, Morelet, J. de C. 1865.

1 Cambodia; ex c. $\Lambda$. Morelet, Esq.

var. compressa, nov.

Long. 60, diam. $44 \frac{1}{2} \mathrm{mil}$.

2 Cochin.China; ex c. P. Bouvier, Esq.

var.

Conf. Reeve, Icon., fig. 35, Pt. Jackson.

1 Loc. ?; ex c. Dr. F. Stoliczka.

\section{Ampullaria dolioides.}

Ampullaria dolioides, Reeve, Icon. 1856, fig. 75, Bombay; fide Ilanley, A. dolioides, $R v$. is from S. America (?).

1 Ceylon; coll. H. Nevill, Esq. $(46,41)$.

\section{Ampullaria moesta.}

Ampullaria moesta, Reeve, Icon. 1856, fig. 92, Ceylon; fide Morelet? $=$ A. borneensis, Phil.var.

4 Balapiti, Ceylon; coll. G. Nevill, Esq. 


\section{Ampullaria lubrica.}

Ampullaria lubrica, Reeve, Icon. fig. 161, 1856, Loc.?.

6 I.uzon; coll. R. Hungerford, Esq.

Scarcely umbilicate, of sombre green colouration. Perhaps an extreme var. of $A$. luzonica, $\mathrm{Kv}$.?

\section{Ampullaria ovata.}

Ampullaria ovata, Olivier, Toy. Emp. Ottom. 1804, Lake Mareotis : fide Bourg., alt. 60, diam.44-apert. long. 42, diam. 27 mil.

1 Oasis of Farâfra, Egypt; ex c. Dr. C. F. Jickeli ("coll. Rholfs").

Long. 47, diam. $37 \frac{1}{2}$; apert. alt. $29 \frac{3}{4}$, lat. 22 mil.

Resembles M. Bourguignat's Pl. X, fig. 11, Moll. nouv. \&c. ; but the spire is even more acutely produced and the aperture smaller.

15. Ampullaria cecillei.

Ampullaria Cecillei, Philippi, Zeits. Mal. 1848, p. 191, Madagascar; $=A$. inops, Morelet, Rev. Zool. 1851.

2 Madagascar; ex c. A. Morelet, Esq.

16. Ampullaria hanleyi.

Ampullaria Hanleyi, Reeve, Icon. 1856, fig. 113, Loc. ?; fide Tristram, P. Z. S. 1863, as “ $A$. adusta, Rv. = sordida, Sow."; fide Martens, $A$. hanleyi, $R v$. is from $S$. America (?).

7 Ranomafana, Madagascar; coll. J. Caldwell, Esq.

var. ( ? distinct species).

P A. simplex, Reeve, fig. 98, Loc.?.

1 Madagascar; ex c. E. Dupont, Esq.

\section{Ampullaria speciosa.}

Ampullaria speciosa, Philippi, Zeits. Mal.1849, p. 18, Zanzibar; Conch.• Cab. II, pl. 11, fig. 2.

1 Locality?; ex c. G. Nevill, Esq.

Sub-Genus POMUS, Humpfrey.

Cat. Mus. Calonnianum, 179\%, and of Mörch, Cat. Yoldi, 1852, type Nerita urceus, Miiller; sect. =Asolene, Orb., Voy. Amer. Merid. 1839, type A. platae, Maton (as A. naticoides). 
18. Ampullaria (Pomus) hopetonensis.

Ampullaria hopetonensis, Lea, Trans. Phil. Soc. V, 1839, pl. XIX, fig. 84, Hopetown, Georgia (? not of Reeve or Philippi); =A. depressa, Say, 1824 (not of Lam. 1822, as "Natica depressa"); = A. paludosa, Say, $N$. H. Diss. II, 1840.

2 Georgia (typical ?); coll. Prof. Shepard.

19. Ampullaria (Pomus) reflexa.

Ampuliaria reflexa, Swainson, Tilloch's Phil, Mag. $L X I, p .37 \%$; var. ?=A. flagellata, Say, N. H. Dissem. II, 1840, Vera Cruz.

3 Carthagena; coll. Rev. E. R. Beadle (as “A. flagellata, Say”).

Fide Strebel, 1873, A. flagellata, Say = malleata, Jonas = reflexa, $\mathrm{Sw} .=$ violacea, Val.

\section{Ampullaria (Pomus) hondurasensis.}

Ampullaria hondurasensis, Reeve, Icon. fig. 15, 1856, Honduras.

1 Guatemala; coll. A. Morelet, Esq. (as "A. reflexa").

1 Loc.?; ex c. G. Nevill, Esq.

\section{Ampullaria (Pomus) gigas.}

Ampullaria gigas, Spix, Test. Bras. 1827, pl. I, fig. 1; Conch.-Cab. II, pl. $X I V$, fig. $1 ;=A$. insularum, Orb. 1839;?= A. canaliculata, Lam. 1822; fide Martens $=A$. haustrum and A. immersa, Reeve.

1 Rio Plata; ex c. A. Morelet, Esq. (as “ $A$. insularum, Orb.”).

var. minor.

Orbigny, l. c., pl. L, figs. 5-6, as "A. canaliculata, Lam. var." (not A. canaliculata, Delessert, as "of Lam., Guadeloupe.").

1 Rio Parana; ex c. A. Morelet, Esq. (sent as “A. canaliculata, Lam.”).

22. Ampullaria (Pomus) fasciata.

Ampullaria fasciata, Lamarck, Hist. An. s. vert. VI, 1822, "l'Inde, des Moluques et des Antilles;" conf. Conch.-Cab. II, pl. XX, fig. 1.

1 Cuba; ex c. Prof. W. Newcomb.

23. Ampullaria (Pomus) australis.

Ampullaria australis, d'Orbigny, Voy. Amer. Mérid. 1839, pl. LI, figs. 3-4.

1 South America; ex c. G. Nevill, Esq. 
24. Ampullaria (Pomus) adusta.

Ampullaria adusta, Reeve, Icon. 1856, fig. 11, Loc. ? (not of Tristram, P. Z. S. 1863).

1 South America; ex c. G. Nevill, Esq.

\section{Ampullaria (Pomus) urceus.}

Nerita urceus, Müller, Hist. Verm.17\%4, p. 1\%4, as " in insulis India edulis."

1 South America; ex c. G. Nevill, Esq.

1 New Granada; ex c. A. Morelet, Esq.

\section{Ampullaria (Pomus) testudinea.}

Ampullaria testudinea, Reeve, Icon. 1856, fig. 114, Loc.? ; fide Martens, should be A. figulina, Spix.

1 Rv. Amazon; ex c. A. Morelet. Esq. (as "A. testudinea").

1 South America; ex c. G. Nevill, Esq.

Reeve's fig. 75, "A. dolioides" is very close to this latter. Spix's fig. of $A$. figulina is very bad; it seems to me it can hardly be this species!

27. Ampullaria (Pomus) martensiana, n. sp.

A. columbiensis, [Sow. Ms.] Reeve, Icon. 1856, fig. 25 and Nevill, Cat. Fasc. E, p. 16 ; not A. columbiensis, [Sow. Ms.] Philippi, Conch.-Cab. $I I, 1851, p l . T$, fig. 5 .

1 S. America; ex c. G. Nevill, Esq.

28. Ampullaria (Pomus) cumingi.

Ampullaria Cumingii, King, Zool. Jour. F.p.344, Taboga, Panama.

1 Panama; ex c. Dr. H. Dohru.

Exactly intermediate in size between Reeve's large form and the smaller one of Conch.-Cab. ; alt. 31 $\frac{1}{2}$, diam. $29 \frac{1}{4}$ mil.

29. Ampullaria (Pomus) decussata.

Ampullaria decussata, Moricand, Mém. Soc. Phys $s_{n}$ Génève, VII, 1836, Brazil.

2 Brazil; ex c. Dr. H. Dohrn.

Neither quite adult. 


\section{Ampullaria (Pomus) crassa.}

Ampullaria crassa, Swainson, Zool. Ill. I, Tol. III, 1822, Loc. ?; Reeve, fig. 5, Brazil.

1 Ecuador; ex c. Dr. H. Dohrn.

Agrees with Reeve's fig. 5, only a little smaller and columella less straight.

\section{var. oblonga.}

Near Reeve's fig. 106, as "A. crassa, oblong convoluted var."

1 Brazil ; ex c. Prof. W. Newcomb (sent as "A. spixi" ).

4 Amazon Rv.; ex c. Prof. W. Newcomb (as " $A$. nubila").

Alt. 221 $\frac{1}{2}$ diam. $19 \frac{3}{4}$ mil.-Imperforate, intermediate between Reeve's figs. 5 and 106 as regards convexity of the whorls and height of the spire. I cannot detect the slightest difference between Dr. Newcomb's specimens, and I believe they must have come from one locality; they do not in the least resemble A. nubila or A. spizi!

\section{Ampullaria (Pomus) platæ.}

Ampullaria Platæ, Maton, Trans. Lin. Šoc., 1S09, R. Plata;=A. naticoides, Orb. 1835 ; sect. Asolene.

1 Rio Plata; ex c. A. Morelet, Esq.

\section{Ampullaria (Pomus) pulchella.}

Ampullaria pulchella, Anton, Verz. Conch. 1839, [sine Hab.] $=A$.

roissyi, 0rb. 1839.

3 Rio Plata; ex c. A. Morelet, Esq.

2 Rio Plata; ex c. Dr. H. Dohrn (as " A. plate").

\section{Ampullaria (Pomus) elegans.}

Ampullaria elegans, d’Orbigny, Voy. Amér. Mérid., 1839, Bolivia, \&c.;= A. peristomata, Orb. 1835 .

4. Bolivia; ex c. G. Nevill, Esq.

The above are all decollated.

1 Brazil ; ex c. Prof. W. Newcomb (as "A. spixii"). 
34. Ampullaria (Pomus) sordida.

Ampullaria sordida, Swainson, Zool. Ill. I, Vol. III, 1822, pl. 142 ; Reeve, Icon. fig. 72, R. Plata.

2 South America; ex c. G. Nevill, Esq.

35. Ampullaria (Pomus) scalaris.

Ampullaria scalaris, d’Orbigny, Voy. Amér. Mérid. 1839, pl. 50, fig. 1-3.

3 Bolivia ; ex c. Prof. E. C. Stearns (as "A. canalis, Orb.”).

35. Ampullaria (Pomus) núbila.

Ampullaria nubila, Reeve, Icon. 1856, fig. 65, Rv. Solomoens.

1 South America; ex c. G. Nevill, Esq. (33, 27).

37. Ampullaria (Pomus) erronea.

Ampullaria erronea, G. Nevill, Cat. Moll. Fasc. E, 1877; Reeve, figs. 90 \& 91, as "A. aperta" [not of Phil.].

1 South America [typej; ex c. G. Nevill, Esq.

38. Ampullaria (Pomus) glauca.

Helix glauca, Linnæeus, Mus. Ulr.1764, p. 667; Conch.-Cab. II, pl. XIT, fig. 4 .

3 South America; ex c. As. Soc., Bengal, and G. Nevill, Esq.

var. guianensis.

Ampullaria guyanensis, Lamarck, Hist. an. s. Vert. VI, 1822, "la Guyane;" Reeve, fig. 85 .

1 British guiana; ex c. A. Morelet, Esq. (as “ A. guianensis”).

var. crocostoma.

Ampullaria crocostoma, Philippi, Conch.-Cab. II, 1851, pl. 12, fig. 3, Caracas; distinct species fide Martens.

2 Caracas; ex c. A. Morelet, Esq. (as " $A$. effusa").

39. Ampullaria (Pomus) geveana.

Ampullaria geveanensis, Deshayes, Lam. Hist. VIII, 1838, p. 541, Loc.?; emend. Phil., Conch.-Cab. II, 1851, pl. VII, fig. \%.

1 South America; ex c. G. Nevill, Esq. 
Sub-Genus $S A U L E A$, Gray.

Proc. Zool. Soc. 1867, type Helix vitrea, Born.

\section{Ampullaria (Saulea) vitrea.}

Helix vitrea, Born, Index Mus. vindob. 1778.

4. Liberia; ex c. Dr. H. Dohrn.

\section{Genus MARISA, Gray.}

Phil. Mag., vol. 63, 1824, p. 274; = Ceratodes, Guilding, Zool.Journ.III, 1828.

\section{Marisa cornu-arietis.}

Helix cornu-arietis, Linnceus, Syst. Nat. X 1758; Conch-Cab. II, pl. XVIII, fig. 1, R. Parana.

1 Loc. ? ; ex c. Dr. F. Stoliczka (labelled "Senegal ").

\section{Marisa chiquitensis.}

Ampullaria chiquitensis, d'Orbigny, Voy. Amér. Mérid.1839, Chiquitos in Bolivia, pl. 4S, figs. 10-11.

1 Bolivia ; ex c. Prof. E. C. Stearns.

var. (? $M$. rotula, Mous.)

1 N. Grenada ; ex c. G. Nevill, Esq.

The last whorl does not increase in breadth near the peristome, as in the typical form.

\section{Genus LANISTES, Montfort.}

Conch. Syst.1810,p. 122, type Helix bolteniana, Chemnitz, not of Humpfrey, Mus. Calonnianum, 1797, type Mytilus discors, Lin. [insufficiently de. scribed $] ;$ sect.$=$ Meladomus, Swainson, Treat. Malac. 1840, p. 340, type Paludina olivacea, Sow.

\section{Lanistes boltenianus.}

Helix Bolteniana, Chemnitz, Conch.-Cab. I, Vol. IX, 1786, p. 89 ; =Cyclostoma carinatum, Olivier, Voy. I804; = Lanistes olivieri, Mtf. 1810; Conch-Cab. II, pl. 6, fig. 4.

3 Locality?; ex c. As. Soc. Bengal. 


\section{var bicarinata.}

a kirkot kursk: coll. Dr. G. Fritsch.

1 laxality : : exe G. Novill, Esq.

rar. depressa.

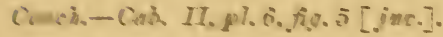

1 Nungula: coll. Dr. G. Fritseh.

\section{Lanistes guinaicus.}

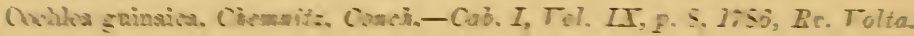

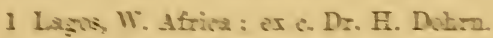

\section{R Ianistes plicosus}

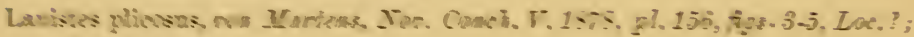

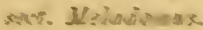

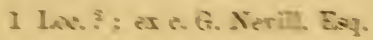

\section{Lanistes orum}

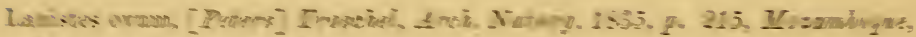

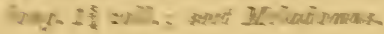

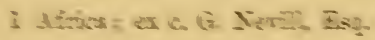

\section{Inmisiss gurparsts}

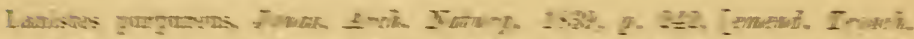

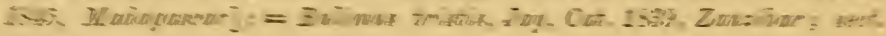
II - menumas.

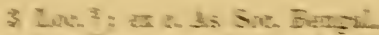

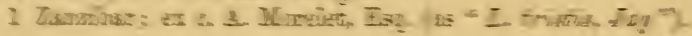

2. Lanisus libgras

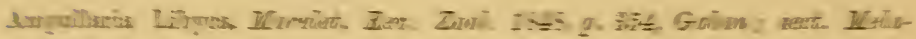
inmorar

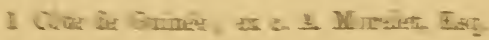




\title{
2. Family VALVA'TIDA, Gray.
}

\author{
Genus VALVATA, Müller.
}

Hist. Verm. II, p. 198, 17\%4, type V. cristata, Müll.; sect. Tropidina, H. and A. Ad., type $V$. tricarinata, Lesueur; sect. Cincinna, Hiibner (fide Mörch), type Nerita piscinalis, Müll. [ = Valvatinella, Betta, 1870] ; sect. Jelskia, Bourg., Syst. Europ. \&c., 18\%\%, type Valvata jelskii, Crosse, J. de C. 1863, Rv. Dnieper.

\section{Valvata cristata.}

Valvata cristata, Müller, Hist. Verm. 17\%4, Denmark.

4. Middlesex ; coll. G. Nevill, Esq.

6 Bayonne; coll. G. Nevill, Esq.

1 Aix-les-Bains; coll. G. Nevill, Esq.

\section{Valvata piscinalis.}

Ncrita piscinalis, Müller, Hist. Verm. 1774, Denmark; $=V$. tolosana, Saint Simon, Ann. Malac., 1870, Toulouse, alt.4, diam. $5 \mathrm{~m}$. ; sinistral var. Jef., Ann. and Mag. 1878, Sunbury and vars. subcylindrica from Grassmere and acuminata from Bristol and Dublin; var. $=V$. depressa, C. Pfr.; sect. Cincinna.

3 England; coll. G. Nevill, Esq.

10 Bayonne; coll. G. Nevill, Esq.

6 Pyrmont, Germany; ex c. Dr. F. Stoliczka.

30 Pangong Lake, Ladak; coll. Dr. F. Stoliczka.

4 Kashmir ; coll. Dr. F. Stoliczka.

7 Canal du Fresquel, Aude : coll. P. Fagot, Esq. (as " V. tolosana").

I find it impossible to separate these typical specimens of $V$. tolosana, from ordinary $V$. piscinalis, even as a variety.

1 Lake Alakul, Siberia; ex c. Dr. H. Dohrn.

\section{3, Valvata contorta.}

Nerita contorta, Müller, Hist. Verm. 17\%4, Denmark.

11 Laacher See, Rhineland; coll. G. Nevill, Esq.

8 Aix-les-Bains; coll. G. Nevill, Esq.

A smaller form than the preceding. 


\section{Valvata stoliczkana.}

Valvata stoliczkana, G. Nevill, Moll. Miss. Yárkand, 1S78, figs. 31-36, Yárkand.

30 Yárkand (type); coll. Dr. F. Stoliczka.

Exceedingly close to $V$. macrostoma, Stein, possibly only a variety; distinguished by being a little more depressed, with more open umbilicus, \&c.

\section{Valvata alpestris.}

Valvata alpestris, Blauner, Ms., Küster, Conch.-Cab. II, 1852, pl. 14, figs. 7-8, Grindelwald.

1 Aix-les-Bains; coll. G. Nevill, Esq.

Perhaps better classed as a var. of $V$. piscinalis! In any case, a well marked form !

12 Tirol ; coll. P. Bouvier, Esq. and P. Joly, Esq.

\section{Valvata eximia.}

Valvata eximia, Servain, Moll. Espagne, 1880, Badajoz : alt.4, diam. 5 mil.

20 Burgos; coll. G. Nevill, Esq.

\section{Valvata virens.}

Valvata virens, Tryon, Proc. Acad. Philad. 1867, pl. I, fig. 7, California.

18 Clear Lake, California; ex c. Prof. W. Newcomb.

\section{Valvata tricarinata.}

Cyclostoma tricarinatum, Lesueur, Ms., Say, Journ. Acad. Philad. I, 1S17, Rv. Delaware; sect. Tropidina.

30 New-York; ex c. Revd. E. R. Beadle and Prof. W. Newcomb.

var. unicarinata.

Valvata unicarinatum, De Kay, New-York Moll. 1840.

12 New-York; coll. Prof. W. Newcomb.

\section{Valvata sincera.}

Valvata sincera, Say, Keating's, Narr. Long's Exp. II, App. 1824, NorthWest Territory; fide Newc. $=V$. humerosa, Tryon.

6 California; ex c. Prof. W, Newcomb. 
10. Valvata macrostoma.

Valvata macrostoma, Stein, Conch. Berlin, 1850.

1 Berlin; ex "Linnæa."

\section{Valvata sauleyi.}

Valvata Saulcyi, Bourguignat, Cat. Moll. coll. Sauley, LSõ3, Damaseus.

4. Damascus; coll. Dr. J. Anderson.

12. Valvata petiti.

Valvatu Petiti, Crosse, J. de C. 1872, "cum Glyptoplysa petiti, in lacu regionis-Grande Vallée des Kaoris-dicte, N. Caléd. mérid."

1 New Caledonia; ex c. P. Bouvier, Esq.

13. Valvata (?) microscopica.

Valvata? microscopica, G. Nevill, Cat. Moll. Fasc. E, 187\%, Pt. Cainingdiam. $1 \frac{1}{9}$ mil.

12 Port Canning (type); coll. G. Nevill, Esq.

15 Port Canning; coll. Colonel G. B. Mainwaring.

14. Valvata (?), n. sp.

3 E. of Pamir Kul, Central Asia; coll. Dr. F. Stoliczka.

There are in the Museum five larval cases belonging to the Fam. Phyganida from New Zealand having all the appearance of a species of Valvata! Also twelve of another species from South India, sent by Colonel Beddome, and five from Port Mahon presented by Mons. Joly!

Sub-Genus LYOGYRUS, Gill.

Proc. Acad. Philad.1863, type V. pupoidea, Gld.

15. Valvata (Lyogyrus) pupoidea.

Valvata pupoidea, Gould, Amer. Journ. Sci., 1 Ser. Vol. XXXVIII, 1810, Massachusetts.

3 Loc.?; ex Mr. Sowerby (c. Lombe Taylor).

Genus (?) HETEROCYCLUS, Crosse.

J. de C. 1872, type H. perroquini, Crosse.

\section{Heterocyclus perroquini.}

Heterocyclus Perroquini, Crosse, J. de C. 1872, "Baie de Sud," N. Caledonia.

4 New Caledonia; ex c. P. Bouvier, Esq. 


\title{
3. Family PALUDINIDA, Gray.
}

\author{
Genus LARINA, A. Adams.
}

P. Zool. Soc. 1854, p. 41, and Gen. Moll.p. 624, type L. strangei, A. Ad.; Nevill (Operculum described, \&c.), Cat. Fasc. E, p. 22; = Robinsonia, H. Nevill, Proc. Ceylon Asiat. Soc., 1869, type R. ceylonica, Nev. and of G. and H. Nevill, J. A. S. B. 1869.

\section{Larina burmana.}

Larina? Burmana, W. T. Blanford, Journ. Asiat. Soc., Bengal, 1866, Irawady Delta; Nevill, Cat. Fasc. E, p. 23 ; var. ?= Robinsonia ceylonica, H. Nevill, 1869.

4 Rangoon River; coll. R. Hungerford, Esq.

1 Port Canning; coll. J. Wood-Mason, Esq.

\section{Larina cincta.}

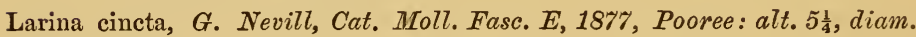
$3 \frac{1}{2}$ mil. and J. A. S. B. 1881, pl. $V$, fig. 6 .

2 Pooree (type); coll. H. Raban, Esq.

I. pusilla, H. Nevill, 1869, from Ceylon, is closely allied.

\section{Genus PALUDINA, Lamarck.}

Extr. Cours anim. sans vert. 1812, type Helix vivipara, Lin.; = "Vivipare," Lam., Phil. Zool. I, 1809; = Viviparus, Mtf. 1810, p. 247 (a misnomer); sect. $=$ Mekongia, Crosse and Fischer, J. de C. 1876, type P.jullieni, Desh.

\section{Paludina contecta.}

Cyclostoma contectum, Millet, Moll. Maine et Loire, 1813; = Nerita vivipara, Müller (not Helix vivipara, Lin.).

4 Potsdam; coll. Dr. E. von Martens.

3 Austria; coll. Dr. F. Stoliczka.

4 England; coll. G. Nevill, Esq.

2 Avignon; ex c. P. Joly, Esq.

\section{Paludina fasciata.}

Nerita fasciata, Müller, Hist. Verm. 1774, "Italy and Saxony"; = H. vivipara, Lin. (a misnomer); = Cyclostoma achatinum, Drap., 1801.

3 England; coll. G. Nevill, Esq.

2 Potsdam; coll. Dr. E. von Marteus.

2 Angoulême; ex c. P. Joly, Esq. 


\section{Paludina contectoides.}

Paludina contectoides, Binney, Smithsonian Misc. Coll. VII, 1865, for $P$. lineata, Küster (not of Val.).

5 Indiana; ex c. Rev. E. R. Beadle.

\section{Paludina subpurpurea.}

Paludina subpurpurea, Say, New Harm. Dissem. II, 1829, “Fox Rv. of the Wabash."

8 Indiana; ex c. Dr. T. Oldham.

4 Ohio ; coll. Prof. W. Newcomb.

\section{Paludina intertexta}

Paludina intertexta, Say, Nero Harm. Dissem. II, 1829, Louisiana.

3 Arkansas; coll. Rev. E. R. Beadle.

\section{Paludina bermontiana.}

Paludina Bermontiana, d'Orbigny, Moll. Cuba, p. 7, 1841.

1 Loc.?; ex c. Dr. F. Stoliczka.

2 Cuba; ex c. Dr. H. Dohrn.

\section{Paludina sublineata [?].}

Paludina sublineata, Conrad, P. Acad. Philad. 1850, Darling River.

1 Australia [?]; ex c. J. Caldwell, Esq.

\section{Paludina ussuriensis.}

Paludina Ussuriensis, Gerstfeldt, Mem. Ac. St. Petersb. 1859 : long. 45-60, diam. 32.40 mil., Mouth of the Ussuri, \&c.

1 River Amoor, Siberia; ex Mr. R. Damon.

\section{Paludina chinensis.}

Paludina Chinensis, Gray, Griffith's Cuvier, 1834, pl. I, fig. 5, China; = P. lecythoides, Benson, Ann. Mag. 1842, Chusan; var. = P. stelmaphora, Bourg., Rev. and Mag.1862, Pekin, long.55 diam. 35m. ; var. = P. ampullacea, Charp. IIs., Conch.-Cab. II, pl. 4, fig. 2-3, Macao

3 Chusan (typical P. lecythoides); ex c. As. Soc. Bengal (coll. Dr. Cantor).

1 China; ex c. A. Morelet, Esq. (as " P. lecythoides").

6 Macao; coll. R. Hungerford, Esq. 


\section{var. ampulliformis.}

Paludina ampulliformis, Souleyet, Voy. Bonite, 1852, River at Touranne.

1 Cochin China (sp. juv.); ex c. A. Morelet, Esq.

5 Basilan, Philippines; coll. Dr. C. Semper.

60 Manwyne, Hotha, Mungla, Nantin and Momien, Yunnan; Mandalay, Burma; coll. Dr. J. Anderson.

There are several distinct forms mixed up in these Yunnan specimens!

subvar. subampulliformis.

3 Tamsui (Formosa); coll. R. Hungerford, Esq.

Exactly resemble the above recorded Nantin specimens, both are less convexly inflated than in Souleyet's type.

var. compacta.

8 Assmungkang (Formosa); coll. R. Hungerford, Esq.

Long. 33, diam. $23 \frac{1}{2}$ mil. A remarkable variety, well characterised by its much less tumid and rounded whorls, which are compressed and of somewhat thicker texture.

\section{var. lecythis.}

Paludina lecythis, Benson, J. A. S. B. V, 1836, Sylhet; Reeve, fig. 12, as "P. ampullacera".

3 Sylhet, types of P. lecythis; ex c. As. Soc. Bengal $(44,38)$.

3 Logtak lake, Mánipur; coll. Colonel H. H. Godwin-Austen.

var. richthofeni.

Paludina chinensis, var. richthofeni, G. Nevill, Cat. Fasc. E., p. 26, 187\%, Java: long. $33 \frac{3}{4}$, diam. 26 mil.

3 Java? (type var.); coll. Baron F. von Richthofen.

var. (?).

1 Thyet Myo; coll. R. Hungerford, Esq.

\section{Paludina bengalensis.}

Paludina bengalensis, Lamarck, Hist. VI, 1822, Bengal; from Cochin China, fide Mor., Ser. Conch.IV; Deshayes, Toy. Belanger, pl. 1, figs. 14-15 [fide Con. Indica, "a rare and distinct var."].

5 Midnapur; ex c. As. Soc. Bengal.

4 Rajmáhal; ex c. Dr. T. Oldham. 
14. Rúrkí and Bírbhúm; coll. J. Wood-Mason, Esq.

2 Siliguri ; coll. Colonel G. B. Mainwaring.

60 Calcutta, Jessor, Chandernagar, \&c.; coll. Colonel G. B. Mainwaring, J. B. Baxter, and G. Nevill, Esq.

13 Jamálpur and Sambalpur; coll. Dr. F Stoliczka and Major M. M. Bowie.

9 Vizagapatam and Madras; ex c. Madras Museum.

20 Mánbhúm and Rániganj; coll. V. Ball, Esq., and Dr. F. Stoliczka.

6 Dacca; coll. J. Willson, Esq. and Dr. Wise.

9 Sind; coll. W. T. Blanford, Esq. and ex c. Karáchí Mus.

9 Derband and Salt Range (Punjab); ex c. Dr. F. Stoliczka.

30 Cachar, Sibságar, and Tezpur (Assam); coll. Dr. F. Stoliczka and Museum Collector.

1 Ganjam (Orissa); coll. V. Ball, Esq.

1 Ferozpur; coll. Capt. R. C. Temple.

5 Sukyti Rv. [Mandi]; coll. A. G. Young, Esq.

subvar. balteata.

P. bengalensis, var, balteata, Benson, J. A. S. B. V, 1836, Sylhet.

30 Silchar, \&c., Cachar; coll. J. Wood Mason, Esq.

subvar. phæostoma, nov.

2 Calcutta (Lindsay St. Tank); coll. J. B. Baxter, Esq.

subvar. elongata.

Paludina elongata, Swainson, Zool. Ill. ser. I, Vol. II, 1822, "Rivers of India"; Reeve, fig. 5.

8 Calcutta (Harrington St. Tank); coll. G. Nevill and J. B. Baxter, Esqrs.

12 Ambálah; coll. Captain R. C. Temple.

Long. 38, diam. 26 mil. A larger and finer form than the one figured by Swainson, as above, with more ventricose last whorl!

\section{subvar. gigantea.}

Paludina gigantea, von dem Busch, Ms., in Reeve, Icon., pl. 1, fig. 7, Bengal.

6 Dinápur; coll. Colonel G. B. Mainwaring $(53,37)$.

subvar. canaliculata, nov.

Paludina bengalensis, var., Nevill, Cat. Fasc. E., p. 2\%, Rániganj.

1 Rániganj ; coll. v. Ball, Esq. 
subspecies doliaris.

Paludina doliaris, Gould, Proc. Bost. Soc. 1843, Vol. I, Tavoy; = "P. benga-

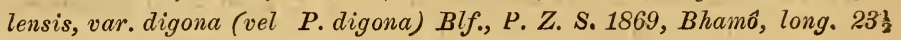
to 32 , diam. $17 \frac{1}{2}$ to $23 \mathrm{mil}$.

30 Mandalay and Bhamô ; coll. Dr. J. Anderson.

40 Myadoung, Shuaygoomyo and Sawaddy; coll. Dr. J. Anderson.

14. Moulmain; coll. Baron F. von Richthofen and 1)r. F. Stoliczka.

5 Pha-thing and Endawyne Lake, Burma; coll. R. Hungerford, Esq.

10 Pegu; coll. W. Theobald, Esq.

\section{subspecies polygramma.}

Paludina polygramma, von Martens, Proc. Zool. Soc. 1860, Siam; =P. lineolata, Mousson, Ms., Reeve, fig. 50, Siam; fide Morelet [not Martens $]=P$. sumatrensis, Dunker, Mal. Blät.1852; = P. speciosa, Desh., Nouv. Arch. X, 1874, Cambodia-"edible."

1 Siam; ex c. A. Morelet, Esq.

7 Cochin-China; ex c. M. M. Morelet and Bouvier.

7 Qualla Kangsa, Perak; coll. E. Townsend, Esq.

subspecies cingulata.

Paludina cingulata, von Martens, Proc. Zool. Soc. 1860, Siam; = P. cochin . chinensis, Mor., Rev. Zool. 1866 ; = P.obscurata, Desh., Nouv. Archiv. Mus. $X, 1874$, Cochin China; $=P$. ingallsiana, Reeve (not of Lea) $;=P$. frauenfeldi, Mor., Journ. de Conch. 1869, Bangkok (not of Desh.).

8 Penang; coll. Dr. F. Stoliczka.

1 Siam; ex c. A. Morelet, Esq. (as "P. cingulata").

1 Siam; ex c A. Morelet, Esq. (as "P. frauenfeld $i$ ").

1 Cochin-China; ex c. A. Morelet, Esq. (as " $P$. cochin-chinensis).

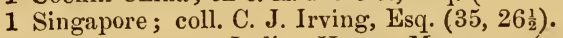

5 Penang; ex c. India House Museum (coll. Cantor, and labelled "P. radula.")

These last are of stouter form, considerably more developed sculpture at base, and slightly less acutely carinate at the periphery than Dr. Stoliczka's Penang specimens.

subvar.

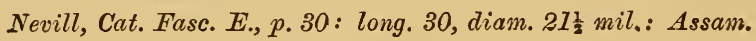

30 Assam; ex c. Drs. T. Oldham and F. Stoliczka.

\section{Paludina oxytropis.}

Paludina oxytropis, Benson, J. A. S. B. $T, 1836$, N. E. Bengal; = P. pyramidata, v. d. Busch, Phil. Abb. I, 1844, Bengal.

3 Mánipur; coll. Colonel H. H. Godwin-Austen. 


\section{Paludina zonata.}

Paludina zonata, Hanley, Ms., Reeve, Icon. fig. 34, Mauritius ; ? = P. ben-

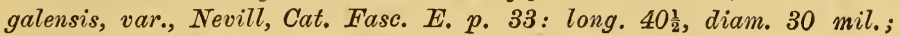
introduced into Mauritius, fide Lienard, Cat. Moll. Mauritius.

16 “Trou d'Eau Douce," Mauritius; coll. G. Nevill, Esq.

\section{Paludina angularis.}

Nerita angularis, Mïller, Hist. Verm. 17\%4, Canton; = P. quadrata, Benson, Ann. \& Mag. 1842, Chusan; = P. polyzonata, Frauenfeld, Philippines.

30 Canton; ex c. R. Hungerford, Esq., Dr. F. Stoliczka, and India House Museum (coll. Cantor).

5 Canton; ex c. Prof. C. Semper.

4 Macao; coll. R. Hungerford, Esq.

The "eye supports" differ from those of true Paludina, fide Benson, J. A. S. B., 1855. Mons. Bouvier has also sent me ten specimens, exactly resembling Canton ones, as from "CochinChina." I fear he has been misled by his correspondent?

\section{Paludina æruginosa.}

Paludina æruginosa, Reeve, Icon., fig. 41, China; fide Martens, Mal. Blät. $1865,=P$. javanica, var. vigorosa $=P$. angularis, var.

2 Shanghai; coll. Dr. C. E. Tonnerre $\left(35 \frac{1}{2}, 24 \frac{1}{2}\right)$.

var. minor.

Long. $25 \frac{1}{2}$, diam. $18 \mathrm{mil}$.

4 Kulangsu, Amoy ; coll. R. Hungerford, Esq,

Very close to Pl. X, figs. 15 to 17 of Kobelt's Fauna Japonica, which are erroneously called $P$. ingallsiana! The Amoy specimens can be easily distinguished from the Japanese form by the subglobose, instead of angulate periphery.

\section{Paludina javanica.}

Paludina javanica, von dem Busch, Phil. Abb. I, 1844, Java; Mousson, Moll. Java, vars. scalaroidea, albomarginata and nigromarginata; var. from Cochin-China, Mor., Ser. Conch. IV, p. 307: long. 31, diam. 18 mil.

1 Samarang; ex c. A. Morelet, Esq.

subvar. minor.

1 Java; ex Mr. Sowerby (c. Lombe Taylor).

Decollate, 3 whorls only remaining: long. 20, diam. $15 \frac{1}{2}$ mil. 


\section{Paludina hamiltoni.}

Paludina Hamiltoni, Metcalfe, Proc. Zool. Soc. 1851, p. 73, Borneo.

1 Borneo; ex Mr. Sowerby (c. Lombe Taylor).

\section{Paludina philippinensis, nov.}

? Vivipara Hanleyi, Frauenfeld, Verz. Paludina, 1864, p. 618, for P. intermedia, Hanley Ms., Reeve, fig. 5\%, 1863, Loc. ? [not of Deshayes]; var. $=P$. carinata, Reeve, 1863, fig. 53, Philippines $[$ not P. carinata, Swainson, 1822 ].

Long. 23, diam. $19 \mathrm{mil}$.

This is a very closely allied from to $P$. javanica; indeed, probably only a geographical race, and perhaps would be better classed as a variety of it!

It can, nevertheless, be readily distinguished by the keel on the last whorls giving the suture a canaliculate appearance, by the less developed spiral sculpture, by the presence of longitudinal, subobsolete plications, as also by its shorter and more decollate form, more convex whorls, the last one subangulate, darker and duller epidermis and blacker peristome, which is more or less angulate below.

Von Martens would not appear to have met with it, as it cannot surely be the form he mentions, Mal. Blät. 1865, p. 148, as a rounded, keelless, extreme variety of $P$. burroughiana! One of my specimens approaches Reeve's fig. 53 (P. carinata) in the more rounded peristome and less distinct subangulation of the last whorl.

7 Majajay, Luzon; coll. R. Hungerford, Esq.

\section{Paludina costata.}

Paludina costata, Quoy, Voy. Astrol. 1832, Celebes; = P. angularis, Auct. [not of Müll.]; Tjiringen (Java), fide Mousson; Nevill, Cat. Fasc. $E, p .33$.

1 Java; coll. Baron F. von Richthofen.

3 Luzon; ex c. A. Morelet and G. Nevill, Esqrs.

8 Zamboanga, Philippines; coll. Prof. C. Semper.

14 Philippines (?); coll. Baron F. von Richthofen.

3 S. Cruz, Luzon; coll. R. Hungerford, Esq.

\section{Paludina ingallsiana.}

Paludina Ingallsiana, Lea, Proc. Acad. Philad. 1856, Siam: long. 0,5?, diam. 0,37 inch [not of Reeve].

var.

1 Siam; ex Mr. Sowerby (c. Lombe Taylor) 


\section{Paludina naticoides.}

Paludina naticoides, Theobald, J. A. S. B. 1865, p. 274, Shan States = P. shanensis, Theob., Catal., 1876, p. 18; Con. Indica, pl. 76, fig. 1; Nevill, Cat. Fasc. E, p. 34.

4 Upper Salween; coll. W. Theobald, Esq.

var. concolor.

Of a light, uniform, olive-green colour; whorls not so exserted as usual, last one less swollen in proportion, more regularly and globosely swollen, with a single, somewhat inconspicuous, raised ridge at the periphery; aperture small, with the outer margin regularly rounded. Long. $25 \frac{3}{4}$, diam. 18 mil.

1 Upper Salween (type var.); coll. W. Theobald, Esq.

var. carinata.

P. naticoides, var. carinata, Theobald, pl. IX, fig. 3, and Con. Indica, pl. 76 , fig. 4.

2 Ava; coll. W. Theobald, Esq.

\section{var. fasciata.}

P. naticoides, var. fasciuta, Theobald, pl. IX, figs. $1 \& 2$, and Nevill, Cat. Fasc. $E$, p. 34 .

7 Upper Salween and Ava; coll. W. Theobald, Esq.

\section{Paludina eyriesi.}

Paludina Eyriesi, Morelet, J. de C. 1865, Cambodia, (not Cochin.China, vide Ser. Con. IV, p. 303); var. $=P$. fischeriana, Mabille and Le Mesle, Rev. Zool. 1866, Battambang.

1 Cambodia; ex c. A. Morelet, Esq.

This Mollusk is edible, fide Journ. de Conch., 1876.

\section{Paludina crassa.}

Paludina crassa, Hutton ms., Benson, J. A. S. B. 1836, "Rv. Gumti at Jônpur" and "greenish eroded var. from Sylhet," for Paludina sp., Hutton, l.c. 1834, Chunar; fide Rv. and Con. Indica $=$ P. obtusa, Troschel; Wood-Mason, Ann. and Mag. 1881 [anatomy].

11 Loc. ? (? typical); ex c. As. Soc. Bengal.

30 Hazrapur, Jessor Dist.; coll. G. Nevill, Esq.

30 Barak Rv., Cachar, t $\uparrow$; coll. J. Wood-Mason, Esq. 
var. tezpurensis.

P. crassa, var. tezpurensis, G. Nevill, Cat. Moll. Fasc. E., 187\%, p. 35, Tezpore: long. $7 \frac{1}{2}$, diam. $15 \frac{1}{2}$ mil.

Exceedingly close to-if indeed specifically distinct froma form sent me by Mons. Morelet from Cambodia, as $P$. hainesiana, Lea!

8 Tezpur, Assam (type var.); coll. Dr. F. Stoliczka.

4 Pegu; ex c. Dr. F. Stoliczka.

\section{Paludina siamensis.}

Vivipara siamensis, von Frauenfeld, Proc. Zool. Soc. 1865 and Ver. Zool.Bot. Gesells. Wien, 1865, Siam : long. 24, diam. 21 mil.

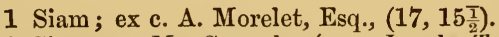

1 Siam; ex Mr. Sowerby (ex c. Lombe Taylor).

Long. 23, diam. 20 mil., and labelled "P. hainesiana."

var. burmanica, nov.

A very small variety; spire short, apex obtuse, with the whorls even less acute and exserted, the last one larger in proportion and more globosely swollen, with the keel round the umbilicus obsolete; aperture more contracted, that is, less dilately expanded; colouration of epidermis and the black peristome exactly similar. Long. 11, diam. $10 \mathrm{mil}$.

1 Prome, on the Irawady (type var.); coll. W. Theobald, Esq.

1 Tenasserim Prov.; ex c. Colonel Godwin-Austen (coll. Mr. O. Limborg).

30 Yaylaymaw, Upper Burma, not quite adult; coll. Dr. J. Anderson.

\section{Paludina hainesiana.}

Paludina Hainesiana, Lea, Proc. Ac. Philad. TIII, 1856, Siam : long. 1, 27, diam. 0, 88 inch; ? var. $=P$. rattrei, $C r$. and $F .[=$ frauenfeldi, Desh. not Mor.].

1 Cambodia; ex c. A. Morelet, Esq. $\left(25 \frac{3}{4}, 20\right)$.

2 Siam; ex c. A. Morelet, Esq. $\left(26 \frac{1}{2}, 20 \frac{1}{2}\right)$.

var, lamarcki.

Paludina Lamarckii, Deshayes, Nouv, Archiv. Mus. X, 1874, Cambodia.

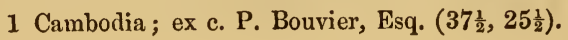


var. sphæricula.

Paludina sphæricula, Deshayes, l. c., Cambodia.

2 Cochin-China; ex c. P. Bouvier, Esq.

These are, I think, the form called $P$. sphcericula; unfortunately they are not quite adult! They present the sharp angulation of the columellar margin at base, said to be characteristic of $P$. rattrei; may this not arise from the form not being adult? Long. $24 \frac{1}{2}$, diam. $20 \frac{3}{4}$ mil.

\section{Paludina lurida.}

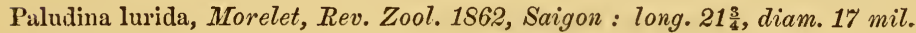

1 Cochin-China; ex c. A. Morelet, Esq.

Very close to typical $P$. crassa, it also much resembles the Tenasserim specimen of $P$. siamensis, var. burmanica, but is larger, with more exserted spire and less obtuse apex.

\section{Paludina dissimilis.}

Nerita dissimilis, Müller, Hist. Term. 1774, Loc. ?; Con. Indica, pl. 77, figs. 2-3 ; P.remossii, "Benson," Phil., Alb. 1846, Meywar (typog. error for P. prcemorsa, Benson, MIs.), and Con. Indica, pl. 77, figs. 8-9, Jounpore, \&c.; juv. =P. carinata, Swainson, Zool. Ill. II, 1822, Ganges ; from Philippines and Siam, fide Morelet, Ser. Con. IV, p. 310.

30 Calcutta, Chandernagar, \&c.; coll. G. Nevill, Esq. and Dr. F. Stoliczka.

18 Nágpur, Derbund and Salt-Range; ex c. Dr. F. Stoliczka.

5 Naini-Tal (?); coll. Dr. T. Oldham.

7 Purneah, Bírbhúm and Benares; coll. Dr. J. Anderson, J. WoodMason, Esq. and Colonel G. B. Mainwaring.

1 Ságar; ex Mr. Sowerby [ex c, Lombe Taylor].

This last a magnificent specimen-long. 271 , diam. 21 mil.scarcely angulate at the periphery, with the white belt subobsolete, was labelled " $P$. olivacea."

subvar. subobtusa, nov.

3 Chándballi; coll. G. Nevill, Esq.

The whorls have not the peculiar, convex shape of var. obtusa. It is probably the $P$. melanostoma of Benson, Ms., but not of Reeve! I described it without a name Cat. Moll. Fasc. E., p. 38 : long. $26 \frac{1}{2}$, diam. 21 mil. : decollate, 3 whorls only remaining. 
subvar. (?).

2 Masulipatam; coll. Dr. F. Stoliczka $\left(30,23 \frac{1}{4}\right)$.

subvar. sindica, nov.

Long. 32, diam. $21 \frac{3}{4}$ mil.; not decollate, 6 whorls, almost colourless, though apparently taken alive as the opercula are still present! Similar specimens were found by Mr. F. Fedden in Katniawar.

10 Sind ; ex c. Karáchí Museum.

subvar. subumbilicata.

12 Ferozpur; coll. Captain R. C. Temple.

Columella straighter than usual, only slightly edged with black; distinctly openly rimate, almost umbilicate; slightly more convex whorls, the last one almost perfectly rounded; belt at periphery less distinct than in typical form, substance of shell a trifle thicker and less brightly coloured.

var. fulva.

Paludina fulva, Reeve, Icon. 1863, fig. 64, from Cambodia.

1 Laos Mns.; ex Mr. Sowerby [ex c. Lombe Taylor].

subvar.

1 Penang; ex c. India House Museum (c. Cantor).

Last whorl a trifle less rounded than in the preceding form, sculpture less distinct, spire more produced; altogether it is scarcely distinguishable from some Indian varieties! In neither of these var. fulva is there any trace of a white belt at the periphery.

var. assamensis, nov.

This is a well marked and characteristic variety, easily distinguished from all the preceding forms by the turreted and remarkably produced spire, the cylindrical instead of convex whorls ; the deep, uniform green colour, the last whorl and aperture much smaller and more contracted in proportion, the latter less everted; periphery subangulate, with the belt obsolete; scarcely rimate; peristome black. 
Long. $22 \frac{3}{4}$, diam. 16 mil. ; slightly decollate.

30 Tezpore, \&c., Assam (type var.); coll. Dr. F. Stoliczka and Dr. T. Oldham.

4 "Jamalpur" (? ? ); ex c. Dr. F. Stoliczka.

16 Silcuri, Cachar; coll. J. Wood-Mason, Esq.

subvar. bhamoensis, nov.

16 Bhamô; coll. Dr. J. Anderson.

An interesting small form of the preceding, with shorter and less produced spire; it is quite unlike all the other Upper Burmese forms brought back by Dr. Anderson and almost induces me to specifically separate from $P$. dissimilis, the forms hereafter recorded as vars. heliciformis, viridis and decussatula, in which case this form would be a variety of true $P$. dissimilis as restricted?

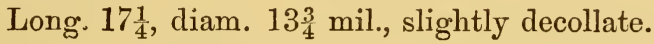

\section{var. obtusa.}

Paludina obtusa, Troschel, Archiv. Nat. 1837, Bengal; Phil., Abb.pl.1, fig. 14, and Kïster, Conch.-Cab. II, pl. VI, figs.8-9 [not P. obtusa of Reeve, or of the Con. Indica].

8 Loc. ?; ex c. As. Soc. Bengal.

4 Naini Tal; coll. Dr. F. Stoliezka.

2 Sambalpur; coll. Major M. M. Bowie.

\section{var. variata.}

Vivipara variata, von Frauenfeld, Ter. Zool.-Bot. Gesells. Wien, 1862, Pondicherry; tupe var. figured, Con. Indica, pl. 115, fig. 8 ; = P. carinata, Reeve, fig. 61 [not of Swainson].

50 Madras; ex c. Dr. F. Stoliczka and Madras Museum, also coll. G. Nevill, Esq.

4. Trichinopoly ; ex c. As. Soc. Bengal.

1 Vizagapatam; ex c. Madras Museum.

1 Loc. ?; ex c. As. Soc. Bengal (marked "P. unicolor").

3 Ceylon; coll. F. Layard. Esq. (sent as "P. melanostoma," Rv.).

10 Ganjam, Orissa ; coll. V. Ball, Esq.

11 Bangalore; coll. G. Nevill, Esq.

5 Kadapā and Kollam; coll. Colonel Beddome.

subvar. ceylonicz.

Paludina cejlonica, Dohrn, Proc. Zool. Soc. 186\%, Ceylon; Con. Indica, pl. 76, fig. 1," and pl. 115, fig. 9, the latter as "P. ceylonica, var. ecarinata."

14. Kandy; coll. G. Nevill, Esq.

6 Madras; coll, G. Nevill, Esq. 
subvar. kutchensis, nov.

Cat. Moll. Fasc. E., p. 40: long, 28, diam. 22 mil. [sine nom.].

9 Kachh; coll. Dr. F. Stoliczka.

subvar. subcarinata, nov.

Cat. Moll. Fasc. E., p. 40 [sine nom.].

10 Moulmain; coll. Dr. F. Stoliczka and Baron F. von Richthofen.

2 Pha-thing, Burma (type var.); coll. R. Hungerford, Esq.

The smoother texiure, less convex whorls and distinct carination are very characteristic; it will probably hereafter have to rank as a well-marked variety. The distinctly open umbilicus separate it from all the forms classed under the next form. Long. $20 \frac{3}{4}$, diam. 16 mil.

\section{subspecies heliciformis.}

Vivipara heliciformis, von Frauenfeld, Proc. Zool. Soc. 1865 and Ver. Zool.Bot. Gesells. Wien, 1865, Central Africa, long. 21, diam. 16 mil., pl. $X X I I$; Con. Indica, pl. $77, f .7$ and 10, Rangoon; fide Hanley, in Con. Indica, types of $P$. heliciformis and $P$. decussatula, Blf. are absolutely identical.

12 Pegu; ex c. Dr. F. Stoliczka (22, 19).

$$
\text { subvar. viridis. }
$$

Paludina viridis, Hanley, Ms., Reeve, Icon. 1862, Loc. ?; Nevill, J.A.S. B. 187\%, p. 31, and Anderson's Zool. Yunnan, 1879, pl. LXXX, fig. 1, Kabyuet.

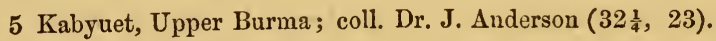

$$
\text { subvar. decussatula. }
$$

“P. dissimilis, var. decussatula (vel P. decussatula)," Blanford, Proc. Zool. Soc. 1869, Ava; Nevill, J. A. S. B. 187\%, p. 31.

14. Bhamô (type subvar.) ; coll. Dr. J. Anderson.

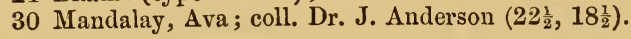

\section{Paludina unicolor.}

Cyclostoma unicolor, Olivier, Voy. Emp. Othom. 1804, Egypt.

2 Locality?; ex c. Asiatic Soc. Bengal.

2 Ist Cataract, Nile; coll. Dr. J. Auderson.

\section{var. subimperforata.}

3 Nile; ex c. Dr. H. Dohrn.

Much less openly umbilicate than the preceding four specimens, consequently still nearer $P$. dissimilis! 


\section{Paludina praerosa.}

Paludina prærosa, Gerstfeldt, Mem. Ac. Imp. St. Petersb 1859: long. 23 to 35, diam. 19 to $26 \mathrm{~m}$. Mouth of the Ussuri.

1 River Amoor, Siberia; ex Mr. Damon.

\section{Paludina auriculata.}

Paludina (Melantho) auriculata, von Martens, Sitz. Ges. naturf. Freunde, Berlin, Jan. 1875; Rv. Siang Kiang, Prov. Hunan: long. 26, diam. 16 mil. (witi two var.); ? sect Mekongia.

2 China; ex c. Prof. E. von Martens.

2 River Siang K iang; ex c. Dr. H. Dohrn (coll. von Richthofen).

30. Paludina jullieni.

Paludina Jullieni, Deshayes, Nouv. Arch. Mus. X, 1878, Cambodia; sect. Mekongia.

1 Cochin-China; ex c. A. Morelet, Esq.

31. Paludina hungerfordiana.

Paludina hungerfordiana, G. Nevill, J. A. S. B. 18s1, Canton: long. 33, diam. 26 mil.; sect. Mekongia.

3 Canton [type]; coll. R. Hungerford, Esq.

32. Paludina martensiana.

Paludina martensiana, G. Nevill, J. A. S. B. 1881, Amoy.

2 Amoy [type]; coll. R. Hungerford, Esq.

33. Paludina hellenica.

Vivipara Hellenica, Clessin, Malak. Blät. 1879.

1 Missolonghi; ex c. P. Joly, Esq.

Sub.Genus LIOPLAX, Troschel.

Gebiss der Schnecken, Lief. 2, 185\%, type P. subcarinata, Say.

\section{Paludina (Lioplax) subcarinata.}

Limnaea subcarinata, Say, Nicholson's Enc. I, 1817, Delaware Rv.

13 Indiana; ex c. Prof. E. C. Stearus, \&c. 
Sub-Genus TULOSTOMA, Haldeman.

Mon. Lymn. Sup. 2, 1841, type P. magnifica, Conrad.

\section{Paludina (Tulostoma) magnifica.}

Paludina magnifica, Conrad, New freshwater shells, U. States, June 1834, Alabama Rv.; = P. bimonilifera, Lea, Trans. Amer. Philos. Soc., August 1834 (read in 1832).

2 Alabama; ex c. Dr. H. Dohrn.

Sub-Genus $C A M P E L O M A$, Rafinesque.

Journ. de Physique, \&c., Vol. 88, 1819, type C. crassula, Raf., from Ohio; $=$ Melantho, Bowditch, Elem. Conch. 1822, type P. decisa, Say.

\section{Paludina (Campeloma) decisa.}

Limnæa decisa, Say, Nicholson's Enc. I, 1817, [sine Hab.]; Delaware Rv. fide Binney.

7 Indiana; ex c. Dr. T. Oldham.

16 Penusylvania; coll. Rev. F. R. Beadle.

4. Massachusetts and Ohio; coll. Prof. E. C. Stearns.

5 New York; coll. Dr. W. Newcomb and ex c. Madras Museum.

A sinistral variety has been recorded.

\section{Paludina (Campeloma) integra.}

Paludina integra, Say, Journ. Aead. Nat. Sci. Philad. II, 1821, Missouri; fide Binney $=P$; decisa, var.

20 New York; coll. Dr. W. Newcomb and Rev. E. R. Beadle.

\section{Paludina (Campeloma) ponderosa.}

Paludina ponderosa, Say, Journ. Acad. Sci. Philad. II, 1821, Ohio.

4 Alabama; coll. Rev. E. R. Beadle.

12 Indiana; ex c. Dr. T. Oldham.

\section{Paludina (Campeloma) genicula.}

Paludina genicula, Conrad, New freshwater shells, U. States, 1834, " Flint Rv., Georgia ;" fide Binney $=P$. decisa, var.

2 Georgia; ex c. Dr. T. Oldham.

40. Paludina (Campeloma) rufa.

Paludina rufa, Haldeman, Mon. Lymn. III, 1841-; fide Binney $=P$. decisa, Say, var.

2 Erie Canal ; coll. Prof. W. Newcomb. 
Genus CLEOPATRA, Troschel.

Gebiss der Schnecken, T, Lief. 2, 1857 [sine descr.], type Cycl.bulimoides, Olivier.

\section{Cleopatra bulimoides.}

Cyclostoma bulimoides, Olivier, Voy. Emp. Othom. II, 1S04, Egypt; fide v. Martens and Jickeli = Melania agyptiaca, in Reeve's Icon. [as "of Benson"].

4 Alexandria; ex c. P. Joly, Esq.

3 Dongola; coll. Dr. G. Fritsch.

\section{Cleopatra verreauxiana.}

Bithinea Verreauxiana, Bourguignat, Rev. Mag. Zool. 1856, Nile; = Paludina cyclostomoides, Küster, Conch.-Cab. II, pl. 7, fig. 6-10, 1852, Egypt.

12 1st Cataract, Nile; coll. Dr. J. Anderson.

subvar. fasciata.

20 Ist Cataract, Nile; coll. Dr. J. Anderson.

I rather doubt if Dr. Jickeli's reasons for adopting M. Bourguignat's name, in preference to Küster's, are valid : there can be no doubt, it seems to me, that the latter can only refer to this species.

\section{Cleopatra aurocincta.}

Cleopatra aurocincta, von Mrartens, Sitz. Ges. nat. Fr. Berlin, 1879, Bagamoyo.

2 Bagamoyo (Somali Land); ex c. Dr. H. Dohrn.

\section{Genus MARGARYA, Nevill.}

J. A. S. B., 1877, p. 30, type M. melanoides, Nevill.

\section{Margarya melanoides.}

Margarya melanoides, G. Nevill, J. A. S. B., 1877, p. 30, Lake Tali in Yunnan and op. c. 1881, pl. 5. fig. 1.

4 Lake Tali, Yunnan (type); ex c. Dr. J. Anderson (coll. Margary).

Genus NEOTHAUMA, Smith.

Proc. Zool. Soc. 1880, type N. tanganyicensis, Smith [Lake Tanganyika].

This remarkable new genus is not yet represented in the Museum. 
Subfamily BENEDICTIINAE, Dybowski.

Von Martens and Clessin consider this subfamily as forming part of the Rissoidce. Despite the characters of the operculum, I can scarcely believe this classification will prove correct.

Genus BENEDICTIA, Dybowski.

Mém. Ac. Petersb. XXII, 1875, type B. fragilis, Dyb.

\section{Benedictia baikalensis.}

Paludina Baikalensis, Gerstfeldt, Mém. Ac. Petersb. IX, 1859, Lake Baikal.

1 Lake Baikal; ex Mr. R. Damon.

Subfamily BYTHINIIN 2 , Troschel (emend.).

Considered by Stimpson and Clessin as a subfamily of the Rissoidce.

Genus BYTHINIA, Leach, Ms.

Gray, Medic. Repos. XV, 1821 [sine descr.]; = Bythinia, Risso, 1826; fide Clessin, "Bythinia," Gray, Philos. Mag. Vol.63, p. 274, type Helix tentaculata, Lin.; fide Martens, "Bithinia" of Fam. Paludinida.

\section{Bythinia cerameopoma.}

Paludina sp. ?, Benson, Gleanings Science, Calcutta, Tol. I, p.363, and Vol. II, 1830, p. 125, as Paludina cerameopoma; also J. A. S. B., 1855 ; $=$ B. ceranospatana, Frauenfeld, Ver. zool..bot. Ges. Wien, 1862; Con. Indica, pl. 38 , figs. 1 and 4 .

30 Calcutta, Hazrapur, Barrackpore, and Rániganj; coll. Dr. F. Stoliczka and G. Nevill, Esq.

30 Assam; ex c. Dr. T. Oldham.

20 Bengal; ex c. As. Soc. Bengal [? typical].

9 Sámbhár Lake, and Sind; coll. Dr. F. Stoliczka.

4 Patna and Rurki ; coll. J. Wood-Mason, Esq., and Colonel G. B. Mainwaring.

30 Ferozpur; coll. Captain R. C. Temple.

subvar. carinulata.

1 Assam; ex c. Dr. T. Oldham.

A remarkably deformed, abnormal specimen.

subvar. gigantea.

1 Calcutta; coll. Dr. F. Stoliczka. 


\section{Bythinia travancorica.}

Bithinia travancorica, Benson, Ann. Mag. VI, 1860, Quilon; Con. Indioa, pl. 38, figs. 2-3.

10 Kollam; coll. Colonel R. H. Beddome.

\section{Bythinia goniostoma.}

Bythinia goniostoma, Hutton ms., Frauenfeld, Fer. zool..bot. Gesellsch. Wien, 1861, p. 610, and Con. Indica, pl.37, fig. 7; = B. lutea, Auct., as "of Gray," Ann. Philos. 1824 [sp. incert., fide Hanl., Con. Ind., p. xviii]; = Paludina pulchella, Conch.-Cab. II, 1852, [as "of Sowerby"], pl. VI, figs. 18-20 [not B. pulchella, Bs.].

30 Calcutta, Pt. Canning, and Rániganj; coll. Dr. F. Stoliczka and G. Nevill, Esq.

30 Madras; ex c. Madras Museum.

1 Galle (?); ex c. Dr. F. Stoliczka.

20 Mánbhum; coll. V. Ball, Esq.

1 Orissa; coll. W. T. Blanford, Esq.

3 Assam; ex c. Dr. F. Stoliczka.

8 Upper India (typical); coll. Captain F. W. Hutton [A. S. B.].

\section{Bythinia pulchella.}

Paludina pulchella, Benson, J. A. S. B. 1836, Sylhet [not Pal. pul. chella of Conch.-Cab. II]; Con. Indica, pl. 33, figs.5.6.

50 Suket, Ambálah, and Ferozpur $\left(10 \frac{1}{2}, 7\right)$; coll. Dr. F. Stoliczka and Capt. R. C. Temple.

20 Kotgarh ; coll. Dr. F. Stoliczka.

20 Rúrki; coll. J. Wood-Mason, Esq.

3 Khási Hills (?); coll. Colonel H. H. Godwin-Austen.

20 Assam; ex c. Dr. F. Stoliczka.

1 Naini Tal; ex c. Dr. F. Stoliczka.

1 Arakan; coll. S. Kurz, Esq.

20 Kollam; coll. Colonel R. H. Beddome.

4 Bombay; ex c. Dr. F. Stoliczka (? coll. Rev. S. B. Fairbank).

2 Salt Lakes; ex c. Dr. F. Stoliczka.

2 Kulu; coll. A. G. Young, Esq.

var. obtusa, nov. (? dist. sp.).

16 Pt. Canning (type var.); ex c. Dr. F. Stoliczka.

var. pusilla, nov.

40 Ferozpur; coll. Capt. R. C. Temple $\left(6 \frac{1}{2}, 4 \frac{2}{3}\right)$.

subvar. canaliculata.

1 Ferozpur; coll. Capt. R. C. Temple. 


\section{Bythinia subpulchella.}

G. Nevill, J. A. S. B. 1881, pl. VI, fig. 12, Kutch: long. 6, diam. 4 mil.

30 Kachh (type); coll. Dr. F. Stoliczka.

2 Morádábád ; ex c. As. Soc. Bengal.

16 Delbi; coll. J. Wood-Mason, Esq.

var. tenuior (? dist. sp.).

$3 \mathrm{Kachh}$; coll. W. T. Blanford, Esq.

\section{Bythinia inconspicua.}

Bythinia inconspicua, Dohrn, Proc. Zool. Soc. 186\%, Ceylon; Con. Indica, pl. 37, figs. 5-6.

1 Ceylon ; coll. F. Layard, Esq. (sent as "B. orcula").

4 Ceylon; ex c. Dr. F. Stoliczka.

6 Loc. ?; ex c. W. Theobald, Esq.

12 Madras; ex c. Madras Museum.

8 Derband ; ex c. Dr. F. Stoliczka.

9 Trichinopoly; coll. H. F. Blanford, Esq. [A. S. B.]

var. (? dist. sp.).

12 Assam (?) ; ex c. Dr. F. Stoliczka.

\section{Bythinia, sp.}

1 Abyssinia (?); coll. W. T. Blanford, Esq.

This specimen was mixed with Abyssinian Vitrina mamillata: can it be the specimen mentioned by Blanford, Zool. Abyssinia, page 472, and which is not forthcoming? It seems to me undistinguishable from the Calcutta form of $B$. lutea.

\section{Bythinia orcula.}

Bythinia orcula, Benson, MIS, Frauenfeld, Ver. zool.-bot. Gesellsch. Wien, 1862, p. 1154 ; Con. Indica, pl. 38, figs. 8-9, Purneah.

50 Rániganj, Pt. Canning, Calcutta, Patna, and Bhaugulpore; coll. Dr. F. Stoliczka and G. Nevill, Esq.

4 Rajmáhal ; coll. Colonel G. B. Mainwaring.

50 Ambálah, Delhi, and Ferozpur ; coll. J. Wood-Mason, Esq. and Capt. R. C. Temple.

5 Assam; ex c. Dr. F. Stoliczka.

subvar.

2 Patna ; coll. Colonel G. B. Mainwaring.

subvar. minor.

3 Orissa ; coll. V. Ball, Esq. 
var. producta (? dist. sp.)

8 Sámbhar and Salt-Lakes; ex c. Dr. F. Stoliczka.

6 Jamalpur; ex c. Dr. F. Stoliczka.

10 Ferozpur; coll. Capt. R. C. Temple $\left(7,5 \frac{1}{4}\right)$.

var. parvula (? dist. sp.)

Morádábád; ex c. As. Soc. Bengal.

Jaunpur ; ex c. India House Museum [as "B. orcula-coll. Cantor"].

var. acuminata (? dist. sp.)

1 Andamans [?]; coll. F. A. de Roepstorff, Esq.

This locality will be most interesting when confirmed.

\section{Bythinia stenothyroides.}

Bythinia stenothyroides, Dohrn, Proc. Zool. Soc. 1867, Ceylon; Con. Indica, pl. 38, figs. 7 and 10.

Extreme forms like the above quoted figure are easily distinguished. Specimens from the same piece of water, however, vary sometimes to an extraordinary degree, in the more or less produced spire; some forms seem to unite the species with $B$. orcula on the one hand and $B$. inconspicua on the other.

50 Madras; coll. Dr. F. Stoliczka, G. Nevill, Esq., and ex c. Madras Museum.

12 Ceylon; coll. F. Layard, Esq.

6 Ceylon; coll. E. L. Layard, Esq. [A. S. B.].

10 Trichinopoly; coll. H. F. Blanford, Esq.

2 Nilgiris ; ex c. India House Museum (sent as “ B. alba, Prideaux").

25 South Arcot and Kollam; coll. Colonel R. H. Beddome.

subvar. biangulata, nov.

1 Madras; ex c. Madras Museum.

var. abnormis, nov.

20 Bangalore; coll. G. Nevill, Esq.

var. (? dist. sp.)

1 Sicrigali [?]; ex c. Dr. T. Oldham.

var. (? dist. sp.)

1 Penang; coll. Dr. F. Stoliczka. 
10. Bythinia, n. sp. [?]

3 Goalundo; coll. Dr. Wise.

11. Bythinia, n. sp.

8 Damotha, Moulmain; coll. Dr. F. Stoliczka.

\section{Bythinia nassa.}

Bythinia nassa, Theobald, J. A. S. Bengal, 1865, Shan States, and op. c. 1870, pl. 18, fig. 8; Con. Indica, pl.37, figs. 8.9.

7 Shan States (typical) ; ex c. W. T. Blanford \& W. Theobald, Esqrs.

13. Bythinia laevis.

Bithinia laevis, Morelet, Ser. Conch IV, 1875, Siam and Cochin.China: long. 10, d. 5 mil.

5 Cambodia; ex c. A. Morelet, Esq.

12 Damotha, Moulmain; coll. Dr. F. Stoliczka and Baron F. von Richthofen.

\section{Bythinia goniomphalus.}

Bithinia goniomphalos, Morelet, Rev. Zool. 1866, " Tay-Niah," Cochin China-long. 14, d. $7 \frac{1}{2}$ mil.; = B. iravadica, Blf., Proc. Zool. Soc.

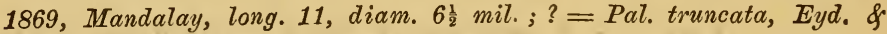
Soul., 1852, "Ganges" [from Sian fide Mts., P. Z. S. 1860].

2 Cochin-China (typical); ex c. A. Morelet, Esq.

2 Siam ; ex c. A. Morelet, Esq. (as "B. siamensis, Lea").

These latter do not agree at all with Lea's figure of $B$. siamensis; they appear to be a rather small form of the preceding. I fancy this is now Mons. Morelet's own opinion, judging from his remarks on this species in Ser. Conch. IV.

30 Mandalay (type of $B$. iravadica), Ava and Kabyuet; coll. Dr. J. Anderson.

4 Thyet-Myo ; coll. R. Hungerford, Esq.

\section{Bythinia moreletiana.}

Bithynia moreletiana, Nevill, J.A. S. B., 187\%, Yaylaymaw; op.c. 1881, pl. VI, fig. 14.

20 Yaylaymaw, in Upper Burma (type) ; coll. Dr. J. Anderson.

16. Bythinia, n. sp. (?).

1 Kurnúl; ex c. Dr. F. Stoliczka. 
17. Bythinia, n. sp.

5 Thyet-Myo; coll. R. Hungerford, Esq. (?)

18. Bythinia evezardi.

Bythinia evezardi, W. T. Blanford, J. A. S. B. 1880, Lonávla (between Bombay and Poona) : long. $3 \frac{3}{4}$, diam. $3 \frac{1}{4}$ mil.; Nevill, op. c. 1881,pl. VI, fig. 13, Mahábaleshvar.

2 Mahábaleshvar ; coll. Rev. S. B. Fairbank.

19. Bythinia badia.

2 Japan [?]; ex Mr. Damon (coll. A. Adams, and labelled " B. badia, A. Ad.").

20. Bythinia striatula.

Bithinia striatula, Benson, Ann. Mag. 1842 and J. A. S. B., 1855, Chusan, "coll. Cantor."

2 China ; ex c. A. Morelet, Esq.

1 Canton; ex c. Dr. F. Stoliczka.

3 Chusan; ex Mr. R. Damon (coll. Cantor).

4. Swatow; coll. R. Hungerford, Esq.

21. Bythinia longicornis.

Bithinia longicornis, Benson, Ann. Mag. 1842 and J. A.S. B. 1855, Chusan, "coll. Cantor."

3 Chusan (typical); ex Mr. R. Damon and As. Soc. Bengal (coll. Cantor).

22. Bythinia tentaculata.

Helix tentaculata, Linnaus, Syst. Nat. $X, 1758, p$. 774; fide Jef., vars. ventricosa, decollata, and excavata, from England.

20 Calais, Biarritz, and England; coll, G. Nevill, Esq.

12 Heidelberg, Pyrmont, and Ireland; ex c. Dr. F. Stoliczka.

5 Potsdam; coll. Dr. E. von Martens.

12 Aix-les-Bains; coll. G Nevill, Esq.

var. kashmirensis, nov.

Long 7, diam. $4 \frac{1}{2}$ mil.

16 Kashmir ; coll. Dr. F. Stoliczka.

2 Srínagar (Kashmir); coll. W. Theobald, Eisq. (?). 
23. Bythinia loachi.

Turbo Leachi, Sheppard, Trans. Lin. Soc. XIV,1823, England; = Paludina ventricosa, Gray, Med. Repos. 1821 (sine descr.); fide Jef., var. elongata, England.

3 England; coll. G. Nevill, Esq.

2 North Italy; ex "Linnæa” (as “ $B$. similis, $\mathrm{Zgl}$. = ventrosa, Gray").

subvar. major.

1 Troitskaja (Siberia) : ex c. Dr. H. Dohrn.

subvar. inflata.

4 Suchorowskaja (Siberia); ex c. Dr. H. Dohrn.

var.

2 Albania; ex “Linnæa" (as “ B. orsinii, Charp.”)

These are certainly not the species figured by Küster as Paludina orsinii, "Charp. Ms., from Ascoli" (Central Italy).

\section{Bythinia troscheli.}

Paludina Troschelii, Paasch, Wiegm. Archiv. 1842, Berlin; = Paludina similis, Boll, Moll. Mecklenb.

1 Aix-les-Bains; coll. G. Nevill, Esq.

7 Kashmir ; coll. Dr. F. Stoliczka.

The latter specimens are all decollate; the whorls are even more convexly rounded than usual.

\section{Bythinia orsinii.}

Paludina Orsinii, Charpentier ms., Küster, Conch.-Cab. II, 1852, Ascoli.

1 Carthagena (Spain); coll. G. Nevill, Esq. $\left(4 \frac{3}{4}, 3 \frac{1}{4}\right)$.

The above original figure agrees exactly; this specimen measures long. $4 \frac{3}{4}$, diam. (vix) 3 mil., and thus agrees fairly with $B$. bourguignati, Palad., from Perpignan, the last whorl of which, however, is less convex; B. meridionalis, Frauenfeld, from Spain, is also somewhat like, but it has the spire less produced, the apex more obtuse, and the aperture more everted.

\section{Bythinia rubens.}

Paludina rubens, Menke, Syn. II, 1830, Sicily ; var. sinistrorsa, Phil., Zeits. Mal. 1844.

2 Sicily; ex "Linnæa".

5 Italy (?); ex c. Prof. H. Troschel. 
var. orientalis.

100 Baalbeck, Rv. Litany, and Damascus; coll. Dr. J. Anderson.

subvar. prætumida.

20 Damascus; coll. Dr. J, Anderson.

subvar. soluta.

3 Damascus; coll. Dr. J. Anderson.

subvar. emaciata.

12 Damascus; coll. Dr. J. Anderson.

27. Bythinia proxima.

Bythinia proxima, von Frauenfeld, Verh. Zool.-Bot., Ges. Wien, 1862, Tirol?

2 Tirol; ex c. P. Bouvier, Esq.

28 Bythinia, n. sp.

12 Vadáli, Bombay Pres.; coll. W. T. Blanford, Esq.

Long. 4, diam. 3 mil.; whorls 4.

29. Bythinia (?) scalaris.

2 Koflein, Austria; ex "Linnæa" (as "Bithinia scalaris, Zgl.).

Seems to me rather a species of Hydrobia (Pseudamnicola). The opercula are unfortunately wanting. $B$. scalaris, Zgl. (fide Frauenfeld), is a variety of $B$. tentaculata.

30. Bythinia philippinensis.

Bythinea philippinensis, G. Nevill, J. A. S. B. 1881, Luzon: long. 8, d. $6 \frac{1}{2}$ mil.

8 S. Cruz, Luzon (type); coll. R. Hungerford, Esq.

31. Bythinia truncatula.

Bithinia truncatula, Morelet, [ubi ?].

8 Madagascar ; ex. c. A. Morelet, Esq. [as "B.truncatula, Morl."]

\section{Bythinia moniliata.}

Bithinia moniliata, Morelet [ubi?].

6 Ste. Marie, Madagascar; ex c. Dr. H. Dohrn [as "B. moniliata, Morl."]. 
Subgenus FOSSARUTUS, Neumayr.

Dentischrift, 1S\$1, tippe F. stachei, Neumayr.

33. Bythinia (Fossarulus ?) marginata.

Turbo marginatus, Chemnitz, Conch.-Cab. $I ;=$ Paludina costigera, Bech, in Conch.-Cub. II, 1552, pl. VII, figs. 1S-19, Bengal, and Con. Indica, pl. 151 , fig. $10 ;=$ Valcata sulcata, Eyd. of Sonl., Voy. Bonite, 1552; species of Fossarulus, fide Brusina, Foss. Moll. Dalmatien, fec, 1874.

30 Karnul, Conjereram, \& S. India ; coll. W. T. Blanford, Esq., and ex c. Madras Mus.

30 Ceylon ; coll. E. L. Layard [A. S. B.] and F. Layard, Esqrs.

subrar. curta.

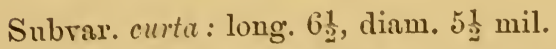

A short form, apparently constant, with the spire less produced or exserted.

Typical, common form : long. $7 \frac{1}{4}$, diam. $5 \frac{1}{2}$ mil.

6 Baugalore; coll. G. Nevill, Esq.

Subgenus HIDROBIOIDES, nor.

Type Bythinia? turrita, Blanford.

34. Bythinia (Hydrobioides) turrita.

Fairbankia? (an Bythinia?) turrita, W. T. Blanford, Froc. Zool. Soc. 1S69, Kyoukpoung-long. 6ł, diam. 3 mil.; Nevill, J. A. S. B., 1SS1, pl. VI, fig. 15 .

1 Kroukpoung, Burma (t.rpe); coll. Dr. J. Anderson.

A very remarkable form, apparently allied to the Byth. meneghiniana, Issel, Att. Sc. Torino XXIII, P1. 1, Figs. 12-13, from Persia.

35. B.thinia (Hydrobioides ?), n. sp.

1 Kachl; ; coll. Dr. F. Stoliczka,

Both this and the preceding may possibly prore to be more nearly allied to Hydrobia than to Bythinia.

Genus STENOTHYRA, Benson.

Ans. Mag. XVII, 1556, type S. deltce Bs., for Nematura, Bs., J. 1.

S. B., $\Gamma, 1836$ (not of Fischer, 1S13, Genus Orthopt.); belongs to Sub. fam. Hydrobiince, fide Stimpson. 


\section{Stenothyra deltæ.}

Nematura deltæ, Benson, Journ. As. Soc. Bengal, $V$, 1836, Calculta, long. 4 to 5 , diam. $2 \frac{1}{2}$ to $3 \frac{1}{2}$ mil.

100 Chándipal, Pt. Canning, Calcutta, and Bhágalpur; coll. Dr. F. Stoliczka, G. Nevill, J. I3. Baxter, and J. Wood-Mason, Esqrs.

Long. $5 \frac{1}{2}$, diam. $3 \frac{1}{2}$ mil. subvar. minor.

20 Pt. Canning; coll. G. Nevill and J. Wood-Mason, Esqr's

Long. 4, diam. $2 \frac{1}{2}$ mil.

subvar. minima.

10 Pt. Canning; coll. G. Nevill and J. Wood-Mason, Esqrs.

Long. $2 \frac{3}{4}$, diam. $1 \frac{3}{4}$ mil.

I can see no difference, even under the lens, between any of the preceding, except in size; there are intermediate forms.

\section{Stenothyra, n. sp.}

1 Chándipal, Bay of Bengal; coll. G. Nevill, Esq.

3. Stenothyra, n. sp.

2 Sonmiáni, Mekran Coast; coll. W. T. Blanford, Esq.

4. Stenothyra glabra.

Stenothyra glabra, A. Adams, Ann. Mag. 1861, Peiho.

24 Swatow, Amoy, and Tokoham (Formosa) ; coll. R. Hungerford, Esq.

5. Stenothyra, sp.

1 Japan? ; ex Mr. R. Damon (as "St. glabra, A. Ad.")

\section{Stenothyra strigilata.}

Stenothyra strigilata, Benson, Ann. Mag. XVII, 1856, Borneo: long. \%, diam. 4 mil.

3 Sarawak; ex c. Prof. A. Issel. 


\section{Stenothyra hungerfordiana.}

Stenothyra liungerforliana, A. Nevill, J. A. S. B. 1880, Andamans, and op, c. 1881, pl. VII, fig. 9.

16 Andamans; coll. Dr. F. Stoliczka and Rev. J. Warneford.

\section{Stenothyra monilifora.}

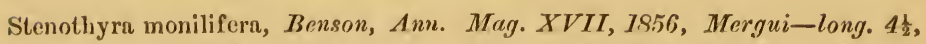
dian. 3 ; Con. Indica, pl. 3\%, fig. 4; ?= S. puncticulalu, Gld., Journ. Boslon Soc. II, 1817, Truvoy-long. $\frac{7}{5}$, lal. + poll. [not of A. Adams, P. Z. S. 1851, Eustern 1sl.-Srom Cochin-China, fide Mor. 1875].

1 Akyab; coll. Dr. F. Stoliczka.

1 Amlerst, Burma; ex c. Dr. F. Stoliczka (labelled "St. subgranosa, Blf.").

var. (? dist. sp.).

1 Penang; coll. Dr. F. Stoliczka.

9. Stenothyra, sp.

1 Shanghai ; ex c. Dr. F. Stoliczka $\left(3_{4}^{3}, 2 \frac{1}{3}\right)$.

\section{Stonothyra glabrata.}

Stenothyra glabrnta, A. Adams, Proc. Zool. Soc. 1851, Penang; Benson, Ann. MIag. YII, 1856-long. 3 to 52, diam. 2 to $3 \frac{1}{4}$ mil. and var. olivacea $=S t$. olivacea, A. Ad., l.c. [sine Mab.].

20 Penang; ex Mr, R. Damon and India Housc Muscum.

\section{Stonothyra polita (?).}

Stenothyra polita, Sowerly MS, A. Adams, P. Zool. Soc. 1851 [loc.?]

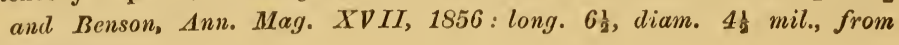
Singapore.

1 Penang; coll. Dr. F. Stoliczka,

\section{Stenothyra fovoolata.}

Stenothyra foveolata, Benson, Ann. Mag. XVII, 1856, long. 5, diam. 34 mil., Sikrigali; Con. Indica, pl. 37, fig 3, "from unique type."

var. minor.

1 Sikrigali, Bengal; coll. 1)r. T. Oldham. 
13. Stenothyra blanfordiana.

Stenothyra blanfordiana, G. Nevill, J. A. S. B., 1880, Chilka Lalke, \&o., and op. c. 1881, pl. VII, fig. 11.

5 Chilka Lake (type); coll. II. F. Blanford, Esq.

30 P't. C'anning; coll. J. Wood-Mason, J. B. Baxter, and G. Nevill, Esqurs.

$$
\text { var. (? dist. sp.) }
$$

10 Arakan; coll. Dr. F. Stoliczka.

1 Penang; coll. Ir. F. Stoliczka.

3 singapore; coll. Dr. F. Stoliczka.

\section{Stenothyra minima.}

Stenothyra minima, Sowerby, Charlesworth's Mag. I, 183\%, long. 0.15 inch, "W. I."; Benson, Ann. Mag. XVII, 1856, long. 2, diam. 1 mil.; Con. Indica, pl. 3\%, fig. 1, Western India.

20 Kathiawar and Bombay; coll. Rev. S. B. Fairbank and F. Fedden, Esisq.

2 Ceylon; coll. II. Nevill, Esq. (as "St. ceylonica, n. sp.").

5 Madras; coll. G. Nevill, Esq.

var. perobvia.

8 Little Rann at Kura, Kathiawar (subfossil); coll. F. Fedden, Esq.

$$
\text { var. (? dist. sp.) }
$$

3 Arakan; coll. Dr. F. Stoliczka.

\section{Stenothyra, n. sp.}

1 Arakan; coll. Dr. F. Stoliczka.

\section{Stenothyra, n. sp.}

1 Penang; coll. Ir. F. Stoliczka.

$$
\text { var. minor (? dist. sp.) }
$$

2 Malacca; coll. Dr. F. Stoliczka.

\section{Stenothyra cingulata.}

Stenothyra cingulata, Benson, Ann. Mag. XVII, 1856, Penang, "coll. Canlor," long. 5 , diam. 3 mil.

1 Penang (typical); ex c. India House Muscum (coll. Cantor). 
18. Stonothyra, sp.

1 Java (?); coll. Baron F. von Richthofen.

Not the species figured by Mousson.

\section{Stenothyra woodmasoniana.}

Stenothyra woodmasoniana, G. Nevill, J. A. S. B. 1880, Pt. Canning, long. $3 \frac{1}{6}$, diam. (vix) 2 mil., and op. c., 1881, pl. VII, fig. 8.

10 Port Cunning (type); coll. J. Wood-Mason, Esq.

\section{Family RISSOIDE, Gray.}

Subfamily $I Y D R O B I I N A E$, Troschel [emend.].

Genus HYDROBIA, Hartmann.

Slurm, Fauna Deulschland, 1821, types II. acula, vilrea, and minula [sine descr.]; lype restricted IIydr. acula, Drap., Fischer, J. de C. 1878; = Paludestrina, Orb. (pars), Voy. Amer. Merid. 1839, p. 381, type P. nigra, Orb. Sc. ; =Ecrobia, Stimpson, 1865, type Turbo minutus, Totten; = Liltorinella, Braun, 1842, and of T'homae, Jahrb. Ver. Naturle. Nassau, II, p. 125, 1845, type Hydr. acula, Drap.;=Peringia, Palad., Ann. Sci. Nalur. 18\%4, type II. ulvae, Mtg.; sect.=Thermhydrolia, Paulucci 18\%8, type Hydrobia aponensis, $v$. Martens.

The animal of IIydrobia [as "Littorinella"] is generically distinct from that of Peringie [as "IIydrobie"], according to Stimpson. Gray, in 'Turton's Manual, 1840, proposed Turbo ulvae, Pennant, as type of the genus, but this cannot, however, be so accepted, because it was not one of Hartmann's original typo species.

\section{Hydrobia ulvæ.}

Turbo Ulvac, Pennanl, Bril. Zool. 1776; fide Jeffreys=Pal.muriatica, Lam. and $[p .310]=$ Cycl. anatinum, Drap. ; fide Mörch, 1871=H. Jeverensis, Schröter, with var. grandis, from Denmark, long. 9 t mil.

20 shoreham and Weymouth; coll, G. Nevill and 'T. l'. Nevill, Esigrs. 
var. obeliscus.

Assiminea obeliscus, Paladilhe, Rev. Mag. Zool. 1860, long. 5, diam. 3 mil., River Macte; emend. Palad., 1874, long. 7, diam. $3 \frac{1}{2}$ mil., as Peringia obeliscus.

14 "Marées de la Macte," Oran ; coll. P. Joly, Escl. (as "Peringia obe. liseus, Pal.").

var. pictonum.

Peringia pictonum, Paladilhe, Ann. Sci. Nat. I, 1874, Charente Inf: and Vendée-long. 6 to $\%$, diam. $3 \frac{1}{2}$ mil.

20 Lake Ossigor [S. W. France]; coll, Marquis de Folin.

Monterosato (1878) considered this variety as a good species : it seems to me scarcely separable. Dr. Kobelt adopts for this species the name of Hydr. starnalis, Bast., "to which he joins ventrosa, Mtg.", as a varicty, but separates IIydr. balticu, Nilss. as a distinct species.

\section{Hydrobia ventrosa.}

'Turloo ventrosus, Montagu, Test. Brit. IT, 1803 ; fide Jeffreys = Cycl. acutum, Drap. 1805 ; fide Mörch, 1871, should be H. slagnalis, Basler $(1 \% 62)=$ ventrosa, Mtg. $=$ baltica, Nilss. $=$ II. octona, $\mathbf{L i n}$.

20 England ; coll. G. Nevill, Esq.

3 Neuwerk (Germany); ex " Linnæa”.

var. baltica [? dist. sp.]

Paludina balthica, Nilsson, Hist. Moll. Sueciae, 1822, Baltic; fide Sars= Cingula minuta, Tolten.

3 Oldenburg; ex "Linnæa" (as "H. baltica, Nilss.").

\section{Hydrobia, sp.}

7 Oran; coll. P. Joly, Esq. (as "H. Oranensis, Bourg.").

var. (? dist. sp.)

6 Oran; coll. P. Joly, Ess. $\left(3 \frac{1}{2}, 1 \frac{3}{4}\right)$.

\section{Hydrobia peraudieri.}

Hydrobia Peraudieri, Bourguignat, Rev. Mag. Zool. 1862, Bislcra, Tuggurt, and fossil at Géryville-long. 6 to 7, diam. $1 \frac{1}{2} \mathrm{mil}$. 
var. minor.

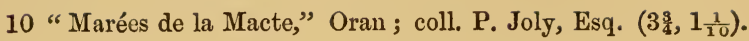

\section{Hydrobia minuta.}

Cingula minuta, Totten ms., Gould, Invert. Massach. 1841, New England; fi e Sars $=$ Pal. balthica, Nilss.

30 Massachusetts ; coll. Revd. E. R. Beadle, \&c.

\section{Hydrobia girardoti.}

Peringia Girardoti, Paladilhe, Ann. Sci. nat. I, 1874, Loire Inf.-long. 6, diam. 3 mil.; fide Kobelt=Hydr. stagnalis, var.

20 Loire Inf.; ex c. P. Joly, Esq. (as “H. muriatica, Lam.”)

\section{Hydrobia brondeli.}

Hydrobia Brondeli, Bourguignat, Rev. Mag. Zool. 1862, Oran, Algiers, Bone \&c.; long. 4, diam. 2 mil. and var. lanceolata, from Tlemcen, Bone \&c.; fide Palad. $=$ Hydr. acuta, Morelet, Moll. Portugal (not of Drap.).

Dr. Kobelt considers both Hydr. brondeli and arenaria, Bourguignat, as varieties of $H y d r$. acuta, Drap.

var. minor.

8 Oran; coll. P. Joly Esq. $\left(3,1 \frac{1}{2}\right)$.

\section{Hydrobia gagatinella.}

Paludina gagatinella, Parreyss ms., Küster, Conch.-Cab. I, 1852, Dal. matia.

3 Pago, Dalmatia; ex "Linnæa" [Frankfurt].

\section{Hydrobia kutschigii.}

Paludina Kutschigii, Kïster, Conch.-Cab. I, 1852, Dalmatia.

2 Scardona, Dalmatia; ex "Linnæa."

\section{Hydrobia consociella.}

Hydrobia consociella, von Frauenfeld, Ver. zool.-bot. Gesellsch. Wien, 1863, Dalmatia.

3 Spalato, Dalmatia ; ex "Linnæa." 


\section{Hydrobia achaja.}

Hydrobia achaja, Clessin, Malak. Blät. XXT, 1878, Eubœa.

8 Eubœa; ex "Linnæa" and P. Bouvier, Esq.

\section{Hydrobia thiesseæ.}

Hydrobia Thiesseæ, Clessin (ubi ?).

8 Eubca; ex "Linnæa" and P. Bouvier, Esq.

Secms to me scarcely separable from the preceding.

\section{Hydrobia aponensis.}

Hydrobia aponensis, von Martens, Wiegm. Arch. 186\%, Abano, for Paludina thermalis, Kïster, \&c. (not of Lin.); sect. Thermhydrobia.

3 Abano (Padua); ex "Linnæa" (as "Hydr. thermalis, Lin.").

Subgenus $B Y T H I N E L L A$, Moquin-Tandon.

J. de C. 1851, p. 239, and Hist. Moll. 1856, type Bulimus viridis, Poiret; sect. $=$ Belgrandia, Bourg., Moll. quart. Seine, 1869, type P. gibba Drap.; sect.$=$ Vitrella, Clessin, 18\%\%, type P. pellucida, Benz; sect.$=$ Frauenfeldia, Clessin, 1879, type F. lacheineri, Clessin; sect. = Maresia, Bourg., Bull. Soc. Toulouse, 187\%, type Hydr. dolichia, Bourg.; sect. = Stimpsonia, Clessin, Mal. Blät. XXV, 1878.

Dr. Kobelt joins the sections DLaresia and Frauenfeldia; but if this is adopted, the former name must have priority. M. de Folin has suggested that the group Vitrella may be the same as his Locardia; M. Bourguignat considers them as distinct: if they prove identical, both names must yield priority to M. Bourguignat's genus Lhotelleria, which is unmistakeably the same as Locardia.

\section{Hydrobia (Bythinella) obtusa.}

Paludinella obtusa, Lea, Trans. Am. Philos. Soc. IX, 1844, Ohio; sect. Stimpsonia.

2 New York ; coll. Prof. E. C. Stearns.

\section{Hydrobia (Bythinella) binneyi.}

Pomatiopsis Binneyi, Tryon, P. Philad. Ac. 1863, Bolinas, California; ? sect. Stimpsonia.

20 Baulines Bay; coll. Prof. E. C. Stearns. 
16. Hydrobia (Bythinella) lanceolata.

Hydrobia lanceolata, Paladilhe, Rev. and Mag. Zool. 1869, St. Jean de Luz.

30 Bayonne; coll. G. Nevill, Esq.

17. Hydrobia (Bythinella), sp.

3 Alsace; ex c. P. Joly, Esq.

var. (?).

3 Alsace; ex c. P. Joly, Esq.

18. Hydrobia (Bythinella) ligurica.

Hydrobia Ligurica, Paladilhe, Rev. MIag. Zool. XIX, 1867, Finale [N. Italy].

1 Alassio ; coll. G. Nevill, Esq,

19. Hydrobia (Bythinella) saxatilis.

Hydrobia saxatilis, Reynies, ms., Dupuy, Moll.Fr. 1851, p. 561, Montauban; fide Paladilhe, of "Lettre à M. Moquin-Tandon, 1843, pl. I, fig. 3.

6 Cantal ; coll. P. Brevière, Esq.

$$
\text { var. [? dist. sp.] }
$$

7 Bayonne ; coll. G. Nevill and Marquis de Folin.

Has a smaller aperture, more produced spire, and less obtuse apex than the preceding. Dr. Kobelt considers it a species of the section Frauenfeldia.

\section{Hydrobia (Bythinella) uzielliana.}

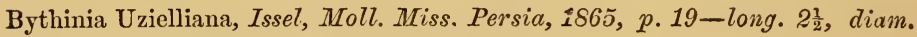
$1 \frac{1}{4}$ mil.-hot-water springs at Kerman.

100 Asupas, Sabestan and Karman (Persia); coll. W. T. Blanford, Esq.

21. Hydrobia (Bythinella) peracuta.

Hydrobia peracuta, Paladithe, Rev. Mag. Zool.1869, "Lyon" and "Nyons" [Switzerland]-long. 3, diam. $1 \frac{3}{4}$ mil.

3 Alsace; ex c. P. Joly, Esq.

var. [? dist. sp.]

3 Alsace; ex c. P. Joly, Essq. 
22. Hydrobia (Bythinella) viridis.

Bulimus viridis, Poiret, Prod. Moll. Aisne, 1801.

9 France ; coll. A. Malherbe, Esq. [A. S. B.]

2 Grasse ; ex "Linnæa."

\section{Hydrobia (Bythinella) elachista.}

Hydrobia elachista, Bourguignat, Rev. Mlag. Zool. 1862, p. 112-long. \$, diam. 2 mil.-Constantine; sect. Frauenfeldia, fide Kobelt.

? Constantine ; ex c. P. Joly, Esq.

\section{Hydrobia (Bythinella) n. sp.}

2 Cheragas, Algeria; coll. P. Joly, Esq.

\section{Hydrobia (Bythinella) 11. sp. [?]}

Conf. Paludina minutissima, Schmidt, Conch.-Cab. II, pl. 13, fig. 26-2\%, "Krain."

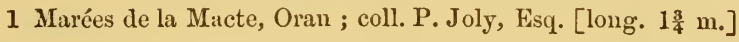

26. Hydrobia (Bythinella) carinulata.

Hydrobia carinulata, Drouet, Moll. Côte d' Or, 186\%, Dijon.

30 Dijon ; coll. G. Nevill, Esq.

27. Hy Arobia (Bythinella) rubiginosa.

Paludina rubiginosa, Boubée, Bull. Hist. Nat. 1833, St. Girons (Ariège); fide Moquin-Tundon $=H y d r$ viridis, Poiret, var. [not Palad.].

30 Ariège ; ex c. P. Joly, Esq.

28. Hydrobia (Bythinella) abbreviata.

Paludina abbreviata, Michaud, Compl. Drap. 1831, Lyons.

20 S. Saulge (Dep. Nièvre); coll. P. Briévière, Esq.

\section{Hydrobia (Bythinella) reyniesi.}

Hydrobia Reyniesi, Dupuy, Hist. Moll. Fr. 1851, Cauterets, \&c.; fide Fischer = Hydrolia canaliculata, Palad. 1869, "près Cierp;" sect. Frauenfeldia, fide Kobelt.

7 Cauterets; coll. G. Nevill, Esq. 
30. Hydrobia (Bythinella) etrusca.

Hydrobia Etrusca, Paladilhe, Rev. Mag. Zool. XIX, 186\%, nr. Florencelong. $2 \frac{3}{4}$, diam. $1 \frac{1}{4}$ mil.

1 Alassio [Liguria]; coll, G. Nevill, Esq.

31. Hydrobia (Bythinella) servainiana.

Hydrobia Servainiana, Bourguiguat ms., Paladilhe, Ann. Malac. 18\%0, st. Jean de Luz, Dep. de l'Herault-long. 3, diam. $1 \frac{2}{3}$ mil.

8 Hamman Sidi Djabellay (Algeria); ex c. P. Joly, Esq. (as “ H. servainiana").

32. Hydrobia (Bythinella) marginata.

Paludina marginata, Michaud, Compl. Drap. 1831, Draguignan [Dept. Var]; sect. Belgrandia.

1 Dep. Ariège; ex c. P. Joly, Esq.

\section{Hydrobia (Bythinella) nana.}

Paludina nana, Terver, Cat. Moll. N. Africa 1839, Bougie \& Guelma; sect. Frauenfeldia, fide Kobelt.

25 Algiers; coll. P. Joly, Esq.

\section{H.ydrobia (Bythinella) glabra.}

Hydrobia glabra, Tryon, Amer. Journ. Conch. I, 1865, Bolivia, "ex c. Nev. comb."

30 Bolivia (typical) ; coll. Prof. W. Newcomb and W. A. Brown, Esq.

\section{Hydrobia (Bythinella) miliacea.}

Hydrobia (Belgrandia) miliacea, G. Nevill, Journ. Asiat. Soc. Bengal, 19s0, Pt. Canning, and op. cit. 1881, pl. VII, fig. 7; sect. Belgrandia.

100 Port Canning, Lower Bengal [type]; coll. G. Nevill, Esq., and Colonel G. B. Mainwaring.

subvar. gibbosula, nov.

1 Port Canning; coll. Dr. F. Stoliczka.

subvar. subangulata, nov.

10 Port Camning; coll. G. Nevill, Esq.

4 Chilka Lake; coll. H. F. Blanford, Esq. 
var. minor [? dist. species],

Hydrobia miliacea, var. minor, G. Nevill, Journ. Asiat. Soc. Bengal, 1880

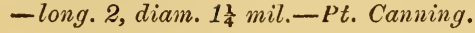

20 Port Canning; ; coll. Dr. F. Stoliczka and G. Nevill, Esq.

36. Hydrobia (Bythinella ?), n. sp. (?)

? Paludinella subsinuata, Dunker, Malak. Blät. 1862, Pondicherry.

50 Lake Negumbo, Ceylon; coll. H. Nevill, Esq. [as “H. ceylanica, n. sp."]

37. Hydrobia (Bythinella ?) n. sp.

4 Sind; coll. W. T. Blanford, Esq.

1 Bombay ; coll. Rev. S. B. Fairbank.

Probably two distinct species.

38. Hydrobia (Bythinella ?) n. sp.

1 Brackish-water Lake, Mauritius; coll. G. Nevill, Esq.

39. H.ydrobia (Bythinella) austriaca.

Paludinella Austriaca, von Frauenfeld, Sitz. K. Akad. Wissens. Wien, XXII, 1862, Lower Austria.

2 Carynthia [Austria]; ex "Linnæa."

40. Hydrobia (Bythinella) dunkeri.

Paludinella Dunkeri, von Frauenfeld, Sitz. $K$. Akad. Wissens. Wien, XXII, 1862.

3 "Biedenkopf " [Germany] ; ex " Linnæa."

41. Hydrobia (Bythinella) lacheineri.

Paludina Lacheineri, Charpentier ms., Küster, Conch.-Cab. II, 1853, Gratz and Laibach; sect. Frauenfeldia.

3 Mantua [Italy]; ex " Linnæa."

2 Venice; ex c. C. F. Ancey, Esq.

42. Hydrobia (Bythinella) schmidti.

Paludina Schmidtii, Charpentier ms., Küster, Conch.Cab. II, 1852, Laibach. 3 Carynthia; ex "Linnæa." 
var. cyclolabris.

Paludinella cyclolabris, Rossmässler ms., v. Frauenfeld, Ver. zool..bot. Ges. Wien, 1863.

2 Hall, Tyrol ; ex c. C. F. Ancey, Esq.

43. Hydrobia (Bythinella) charpentieri.

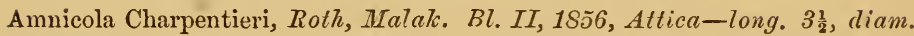
$3 \mathbf{L}$ mil.

2 "Parnés," France [?] ; ex "Linnæa."

44. Hydrobia (Bythinella) opaca.

Paludinella opaca, Ziegler ms., von Frauenfeld, Sitz. K. Akad.Wissens. Wien, XXII, 1862.

2 Camiola [Austria]; ex " Linnæa."

45. Hydrobia (Bythinella) lata.

Paludinella lata, von Frauenfeld, Ver. K. zool.*bot. Ges. Wien, 1863, Hungary.

2 Kaschau [Hungary]; ex "Linnæa."

46. Hydrobia (Bythinella) parvula.

Paludina parvula, Hutton, J. Asiat. Soc. Bengal, 1849, Kojuck Pass, Chummun-anfr. 4, long. $\frac{3}{16}$ th inch; Con. Ind.pl.151, fig. 8, Afghanistan, also Ahmednugger; probably =Bythinia globula, Lea, 1856, "India and tanks at Calcutta" [?]

I have not seen any species of the genus from Afghanistan or the North-West Frontier.

The species is not represented in the Museum.

47. Hydrobia (Bythinella) gibba.

Cyclostoma gibbum, Draparnaud, Hist. Moll. France, 1805 [sine Hab.]; sect. Belgrandia.

3 South France; ex "Linnæa."

48. Hydrobia (Bythinella) californica.

Hydrobia Californica, Tryon, Amer. Journ. Conch. I, 1865, Oakland, \&c.

15 Oakland, California; coll. Prof. W. Newcomb.

49. Hydrobia (Bythinella) dolichia.

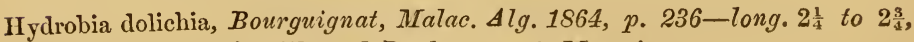
diam. 1 mil.-Géryville and Boghar; sect. Maresia.

5 Edough [Bône]; ex c. P. Joly, Esq. 
Sub-Genus TRIONIA, Stimpson.

Smithsonian Misc. Coll. VII, 1865, type T. elathrata, Stimpson.

50. Hydrobia (Tryonia) protea.

Amnicola protea, Gould, Proc. Boston Soc. $T, 18 \tilde{5}$, Colorado Desert.

18 Desert, E. of California; coll. Prof. E. C. Stearns.

Sub-Genus PSEUDAIINICOLA, Paulucei.

Mater. Malac. Ital. 18\%8, type Taludina similis, Drap. [for Amnicola, Authors, not of Gould and Hald.].

51. Hydrobia (Pseudamnicola) similis.

Cyclostoma simile, Draparnaud, Hist. Moll. Fi. 1S05 [sine Hab.]; fide Jeffreys, Hydr. anatina, Auct. [not of Poiret or Drap.].

2 Boufarick [Algiers]; coll. P. Joly, Esq.

3 England; ex c. G. Nevill, Esq.

\section{Hydrobia (Pseudamnicola) dupotetiana.}

Paludina Dupotetiana, Forbes, Ann. Mag. 1838, Bougie.

30 Maison Carrée [Algiers]; coll. P. Joly, Esq.

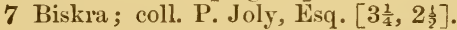

6 Boghar; coll. P. Joly, Esq. [as "Hy. lutea"].

53. Hydrobia (Pseudamnicola) subproducta.

Hydrobia subproducta, Paladithe, Rev. Mag. Zool. 1866, Catalonia and Pyrénées Orientales, "errata," p.40, for Hy. spirata, Paladilhe, l. c. [not of Requien].

10 Cartagena [Spain]; coll. G. Nevill, Esq.

54. Hydrobia (Pseudamnicola) macrostoma.

Paludina macrostoma, Kïster, Conch.-Cab. II, 1853, Athens.

2 Chalcis [Greece]; ex "Linnæa."

55. Hydrobia (Pseudamnicola) seminula.

Amnicola semiuula, Charpentier ms., von Frauenfeld, Ter. zool.-bot. Ges. Wien, 1863, p. 1027, Arcadia-long. 1·5, diam. 1.3 mil.

8 Eubœa; ex "Linnæa” and P. Bouvier, Esq.

\section{Hydrobia (Pseudamnicola) negropontina.}

Amnicola negropontina, Clessin, Malak. Blät. XXV, 1878, Euboea.

4. Eubœa ; ex "Linnæa." 


\section{Hydrobia (Pseudamnicola) vestita.}

Amnicola vestita, Bénoit ( $u b i$ ?).

4 “"Madonée," Greece ; ex "Linnæa."

\section{Hydrobia (Pseudamnicola) conovula.}

Amnicola conovula, von Frauenfeld, Ver. zool.-bot. Gesellsch. Wien, 1863, p. 1026, Pago, \&c.

3 Pago; ex "Linnæa" [as "Amnicola" sp.]

2 Dalmatia; ex "Linnæa" [as "Lithoglyphus" sp. !]

\section{Genus AMNICOLA, Gould and Haldeman.}

Sup. Monogr. Limniades, p. 3, 1840; fide Gould, Invert. Massach. 1841, p. 228, type A. porata, Say; genus of subfam. Fluminicolinae, fide Clessin, 1880.

\section{Amnicola (?) petenensis.}

Amnicola petenensis, Morelet, Test. nov. II, 1851, Central America.

4 Yucatan [typical]; coll. A. Morelet, Esq.

\section{Amnicola (?), sp.}

1 Maranon; coll. A. Morelet, Esq.

Labelled "Assiminea atomus, Mhlf."; it is not, however, Amnicola atomaria, Mhlf., as figured by Kuster.

\section{Amnicola (?) succinea.}

Paludina succinea, Pfeiffer, Wiegm. Arch. VI, 1840, Cuba; fide von Frauenfeld sp. of Assiminea.

1 Cuba; ex c. Dr. F. Stoliczka.

\section{Amnicola pallida.}

Amnicola pallida, Haldeman, Mon. Limniades, Pt.4, 1842, Lake Champlain. 16 New York; coll. Prof. E. C, Stearns.

\section{Amnicola cincinnatiensis.}

Paludina cincinnatiensis, Anthony, Journ. Boston Soc. III, 1840, Cincinnati.

18 New York; coll. Prof. W. Newcomb and E. C. Stearns.

\section{Amnicola limosa.}

Paludina limosa, Say, J. Ac. Philad. I, 181\%, Rv. Delaware, \&c. 50 New York; coll. Prof. W. Newcomb, \&c. 


\section{Amnicola granum.}

Paludina grana, Say, J. Ac. Nat. Sci. II, 1822, Pennsylvania.

40 Michigan; coll. W. A. Brown, Esq.

\section{Amnicola sayana.}

Amnicola sayana, Anthony ms., Haldeman, J. Ac. Nat. Sci. Philad, VIII, 1842, Cincinnati; = Cyclostoma cincinnatiense, Lea, P. Amer. Philos. Soc. 1840.

30 Ohio; coll. Prof. W. Newcomb and W. A. Brown, Esq.

The radula is very different from that of the type species, comp. Clessin, Mal. Bl. 1880 !

\section{Amnicola badia.}

Amnicola badia, Gould, P. Boston Soc. III, 1848, BanksPen., New Zealand.

14. Lake Ellmere, New Zealand; ex c. Dr. F. Stoliczka.

2 New Zealand; coll. J. Caldwell, Esq.

\section{Amnicola antipodarum.}

Amnicola antipodarum, Gray, Dieff. Travels, p. 241, 1S50, N. Zealand; Smith. Moll, Erebus and Terror, pl. 6 ; ? sp. of Pomatopyrgus.

4 Lake Letitia, N. Zealand; ex c. Dr. F. Stoliczka.

11. Amnicola panamensis.

Hydrobia Panamensis, Ancey [in litt.].

4. Panama; ex c. C. F. Ancey, Esq.

Subgenus POMATOPYRGUS, Stimpson.

Amer. J. Con. I, 1865, and Smithsonian Misc. Coll. VII, 1855, type Ielania corolla, Gould.

\section{Amnicola (Pomatopyrgus) corolla.}

Melania corolla, Gould, P. Boston, Soc. II, 1S4\%, N. Zealand; = A. cumingiana, Fischer, J. de C. 1859, N. Zealand; var. ? = A. salleana, Fischer, l. c., N. Zealand.

2 Locality?; ex c. G. Nevill, Esq.

\section{Amnicola (Pomatopyrgus) coronata.}

Paludina coronata, Pfeiffer, Wiegm. Arch. I, vol. VI, 1840, No. 76, Cuba; $=$ Paludestrina candean $\alpha$, Orb., Moll. Cuba, 1841; var. ? = Paludina 
crystallina, Pfr., l. c. No. $\% \%$, and Melania spinifera, Adams, $P$. Bost. S. III, 1851, Jamaica; fide Shut. \&c., var. s. $=$ A. anthracina, Mighels, P. B. S. 1845, = P. Jamaicensis, Adams, = P. cisternicola, Morelet; fide v. Martens, $1873=$ crystallina $=$ candeana $=$ cisterni$\operatorname{cola}=$ ornata.

10 Guadaloupe; ex c. Prof. W. Newcomb (sent as "A. candeana").

2 Jamaica; ex "Linnæa" (as " $A$. candeana, Orb.").

1 Portorico; ex Mr. G. Schneider.

\section{Amnicola (Pomatopyrgus) ornata.}

Amnicola ornata, Morelet, Test. nov. II, 1851, Republic San Salvador ; fide Fischer, $1860,=$ A. coronata, var.

6 Guatemala [typical]; coll. A. Morelet, Esq.

\section{Amnicola (Pomatopyrgus) crystallina.}

Paludina crystallina, Pfeiffer, Wiegm. Arch. I, 1840, No. 77, Cuba; Conch.Cab. II, pl. X, fig. \%, 8; fide Fischer, 1860, A. coronata Pfr., var. inermis.

2 Guadaloupe; ex c. Prof. W. Newcomb (mixed with A. coronata).

1 Portories; ex Mr. G. Schneider.

I cannot help thinking that Shuttleworth and others have confounded two distinct, though variable, forms, living together on most of the West Indian Islands.

\section{Amnicola (Pomatopyrgus) jamaicensis.}

Paludina jamaicensis, C. B. Adams, P. Boston Soc. 'III, 1851, Jamaica; A. coronata, var. fide Shut., \&.c.

10 Jamaica; ex c. Dr. H. Dohrn.

7 Jamaica; ex c. Prof. W. Neweomb.

I cannot bring myself to believe this a mere variety of $A$. coronata.

\section{Genus COCHLIOPA, Stimpson.}

Am. J. Conch. I, 1865, and Smithsomian Misc. Coll. VII, 1865, type Amnicola rowelli, Tryon; genus of subfam. Fluninicolina, fide Clessin 1880.

Not in the Museum collection.

\section{Genus FLUMINICOLA, Stimpson.}

Smithsonian Misc. Coll. VII, 1865, type Pal. nuttalliana, Lea; genus of subfam. Fluminicolina, fide Clessin, 1880. 


\section{Fluminicola nuttalliana.}

Paludina Nuttalliana, Lea, Trans. Amer. Philos. Soc. VI, 1839, Columbia River, Oregon.

8 Oregon; coll. Profs. W. Newcomb and E. C. Stearns.

\section{Genus GILLIA, Stimpson.}

Amer. J. Conch. I, 1865, and Smithsonian Mrisc. Coll. TII, 1865, type Melania altilis, Lea; genus of subfam. Fluminicolina, fide Clessin, 1880.

\section{Gillia altilis.}

Melania altilis, Lea, Proc. Amer. Philos. Soc. II, 1841, S. Carolina.

2 Potomac Rv.; coll. Prof. E. C. Stearns.

\section{Gillia integra.}

Melania integra, Say, N. H. Dissem. II, 1S29, Ohio Rv.; Somatogyrus sp. fide Binney, 1865 .

4. United States; coll. Rev. E. R. Beadle.

1 Florida; ex Mr. G. Schneider.

\section{Genus SOMATOGYRUS, Gill.}

P. Philad. Acad. 1863, type Amnicola depressa, Tryon; genus of sulfam. Fluminicolina, fide Clessin, 1850.

\section{Somatogyrus isogonus.}

Melania isogona, Say, N. II. Dissem. II, 1829, Louisville.

6 Kentucky and N. York; coll. Rev. E. R. Beadle and E. C. Stearns.

\section{Genus LITHOGLYPHUS, Ziegler, ms.}

C. Pfeiffer, Deutsch. MIoll. III, 1828, type L. naticoides, Fér. [fide Stimpson, of Hartmann, 1821]; genus of subfam. Fluminicolina, fide Clessin, 1880.

\section{China.}

There is a Lith. litiputanus, Gredler, Jahrb. 1881, from

\section{Lithoglyphus prasinus.}

Lithoglyphus prasinus, Kokeil ms., F. J. Schmidt, Conch. Krain, 1847, and Küster, Conch.-Cab. II, 1852, Carynthia.

1 Klagenfurt [Carynthia]; ex "Linnæa." 


\section{Lithoglyphus naticoides.}

Lithoglyphus naticoides, Férussac ms., C. Pfeiffer, Deutsch. Moll. III, 1828, Austria [fide Bourg. not of Küster].

3 Marosch river, Transylvauia ; ex c. Dr. T. Oldham.

\section{Lithoglyphus martabanensis.}

Lithoglyphus martabanensis, Theobald, J. Asiat. S. Beng. 1870, Prov. Martaban-" with sp. of Paludina, \&c."

3 Near Moulmein [typical ?]; ex c. Dr. F. Stoliczka.

I consider this undoubtedly atrue Lithoglyphus. It bears considerable resemblance to some of the forms that have been described as Jullienia.

\section{Lithoglyphus fuscus.}

Lithoglyphus fuscus, Ziegler, ms., C. Pfeiffer, Deutsch. Moll. III, 1828, Krain.

12 Mitrovitch [?]; ex c. Dr. F. Stoliczka.

\section{Lithoglyphus pannonicus.}

Lithoglyphus pannonicus, von Frauenfeld, Ter. K. zool.-bot. Ges. 1865, Hungary.

2 Hungary; ex "Linnæa."

\section{Lithoglyphus fluminensis.}

Lithoglyphus fluminensis, Sadler ms., Schmidt, Conch. Krain, 1847 [as of Lang], and Küster, Conch.-Cab. II, 1852, pl.12, fig.18-21, Krain; fide von Frauenfeld ? = L. naticoides, var.

2 Carynthia; ex "Linnæa."

\section{Lithoglyphus pygmæus.}

Lithoglyphus pygmæus, von Frauenfeld, Ver. K. zool.-bot. Ges. 1863, Croatia.

4 Crahova, Dalmatia ; ex "Linnæa" and P. Joly, Esq.

\section{Lithoglyphus [?] tricostatus.}

Lithoglyphus tricostatus, Brot, J. de C. XV, 1867, River Uruguay, Prov. of Entre-Rios, "allied to L. buschii, Dkr. and L. [Paludestrina] peristomata, Orb."

2 South America; ex c. Dr. F. Stoliczka. 
Seems to me to belong to an altogether different group from the European species.

Subgenus SPEKIA, Bourguignat.

Descr. esp. Moll.1879, type Lithoglyphus zonatus, Woodward.

\section{Lithoglyphus (Spekia) zonatus.}

Lithoglyphus zonatus, Woodward, P. Zool. S. 1859, Lake Nyanza.

1 Lake Tanganyika; ex Mr. R. Damon.

Subgenus JULLIENIA, Crosse and Fischer.

J. de C. XXIV, 1876, type Melania flava, Deshayes, Cambodia.

Not represented in the Museum.

Subgenus LACUNOPSIS, Deshayes and Jullien.

Nouv. Arch. Mrus. X, 1874, type L. Jullieni, Deshayes, Cambodia [as Genus].

Lithoglyphus martavanensis, Theobald, may possibly prove to belong to this group, but I think this will prove to be not the case.

Not represented in the Museum.

\section{Genus EMMERICIA, Brumati.}

Foss. Moll. Dalmatien, 1874, type Pal.patula, Brum.; genus of sulfam. Emmericince, fide Clessin, 1850.

\section{Emmericia patula.}

Paludina patula, Brumati, Cat. Conch. Monfalcone, 1838, and Küster, Conch.-Cab.II, pl. 8, fig. 15-22.

var. expansilabris.

Conch.-Cab. II, 1852, pl. 8, fig. 18-22, as Pal. patula, Brum.=Pal. expansilabris, Mühlfeld, in litt.

2 Obbrowazzo, Dalmatia ; ex "Linnæa."

M. Bourguignat in his Monograph of the genus, p. 89, considers this form-doubtless correctly-as a distinct species. 
Genus PACHYDROBIA, Crosse and Fischer.

J. de C. XXIV, 1876, type P. paradoxa, Cr. and F.; genus of Fam. Rissoida, fide Martens; fide Poirier, J. de C.1881, belongs to the Hydrobince, next allied to Gillia.

\section{Pachydrobia parva.}

Pachychilus parvus, Lea, P. Ac. Nat. Sci. Philad. IIII, 1856, Siam; = Melania crassilabrum, Reeve, Icon. 1860; = Paludomus cyanostomus, Morelet, J. de C. 1864, Siam.

4 Cambodia; ex c. A. Morelet, Esq.

\section{Pachydrobia paradoxa.}

Pachydrobia paradoxa, Crosse and Fischer, J. de C., 1876, Cambodia.

1 Locality? ; ex "Linnæa."

Genus HEMISTOMIA, Crosse.

J. de C. 1872, type Hemistomia caledonica, Crosse.

\section{Hemistomia caledonica.}

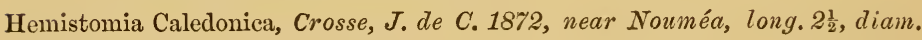
$\frac{3}{4}$ mil.

2 New Caledonia; ex c. P. Bouvier, Esq.

\section{Genus TRICULA, Benson.}

Calcutta J. Nat. Hist. 1842, type T. montana, Benson; fide Stimpson, Stoliczka and Blanford, belongs to Fam. Rissoide.

\section{Tricula montana.}

Tricula montana, Benson, Calc. J. Nat. Hist. 1842, and Ann. MIag. 1862, Bhimthal, at 4,000 ft., Kumaon; Con. Indica, pl.155, fig. 1.

10 Nynee Thal; coll. Dr. F. Stoliczka.

7 Bhimthal [? typical]; ex c. Asiatic Society, Bengal.

var. curta, nov.

2 Jhiri Valley, at 3,000 feet [N. Cachar] ; coll. Colonel H. H. GodwinAusten.

Genus BAIKALIA, von Martens [emend.].

Jahrb. Mal. Ges. 1876, for Limnorea, Dybowski, 1878 [not of Leach, \&(.)] type B. angarensis, Gerst.; sect. = Liobaikalia, MLartens, Jahrb. 1S76, type B. stiedae, Dyb.; sect, $=$ Dybowskia, Dall, P. B. S. 1876, type B. ciliata, Dyb. 
Clessin, in the Nachrichtsblatt for 1878 , p. 126 , considers Lartetia, Bourguignat, as a close ally, and that both should constitute a family between the Rissoidæ and Melaniidæ; in 1880 he classes the group as part of the latter.

\section{Baikalia stiedæ.}

Leucosia Stiedæ, Dybowski, Acad. Imp. St. Petersburg, 18\%5, Lake Baikal; sect. Liobaikalia.

1 Lake Baikal; ex Mr. R. Damon.

Genus TRACHYBAIKALIA, von Mrartens [emend.].

Jahrb. Mal. Ges. 1876, for Ligea, Dybowski, 18\%5, type T. costata, Dyb. [not of Fabricius, genus Crust.]; sect. = Godlewskia, Crosse and Fischer, J. de C. 1879, type T. turriforimis, Dyb.; belongs to Fam. Melaniida, fide Clessin.

Not represented in the Museum.

Subfamily SKENEIN EN, Stimpson.

Genus SKENEA, Fleming.

IIist. Brit. Anim. 1828, type Turbo planorbis, Fabr.

\section{Skenea planorbis.}

Turbo planorbis, O. Fabricius, Fauna Greenland, 1780.

8 England; coll. G. Nevill, Esq.

10 England; ex Mr. R. Damon.

Subfamily POMATIOPSINAE, Stimpson.

Genus POMATIOPSIS, Tryon.

P. Philad. Ac. 1862, p. 452, type Paludina lapidaria, Say.

\section{Pomatiopsis lapidaria.}

Paludina lapidaria, Say, J. Ac. Nat. Sci. I, 1817, Missouri to Michigan.

18 Niagara Falls; coll. Prof. E. C. Stearns.

12 Michigan; coll. Prof. W. Newcomb.

\section{Pomatiopsis intermedia.}

Pomatiopsis intermedia, Tryon, Am. J. Conch. I, 1865, S. Z. Oregon.

4. Oregon; coll. Prof. W. Newcomb. 
Subfamily ASSIMINEINZE, Nevill.

Genus ASSIMINEA, Leach, ms.

Gray, Lond. Medic. Repos. 1821 [sine descr.], Fleming, Brit. Anim. 1828, and Man. Turton, 1840, type A.grayana, Leach; sect. = Paludinella, Pfr., Wiegm. Arch. I, 1841, p. 22\%, type Helix littorina, Delle Chiaje, Sicily.

There is a species from the Comoro Islands, Assiminea parvula, Morelet, J. de C. 1877.

\section{Assiminea pinguis.}

Assiminea pinguis, von Martens, Ann. Mag. XVII, 1866, Macao, "in a muddy inlet"-long. 6, diam. 4 mil.

10 Canton River [mouth of-]; coll. R. Hungerford. Esq.

Scarcely separable from Assiminea brevicula.

\section{Assiminea grayana.}

Assiminea grayana, Leach ms., Fleming, Brit. Anim. 1828, near Greenwich.

6 Mouth of the Thames; coll. E. Webster, Esq.

3. Assiminea francesiæ [emend.].

Turbo Francesi, Gray, Wood's Ind. Test. Sup. 1828, E. Indies; $=A$. fas. ciata, Benson, Zool. J. 1835, p. 463.

16 Zwagabin, near Moulmein; coll. Dr. F. Stoliczka.

16 Chandernagore, Calcutta, and Pt. Caming; coll. Colonel G. B. Mainwaring and G. Nevill, Esq.

The original figure of the type has a conspicuous broad white belt at the periphery; specimens with this band are everywhere much more uncommon than those without it.

subvar, subconcolor.

50 Calcutta; coll G. Nevill, Esq.

Long. 10, diam. 6 mil.

subvar. multifasciata.

50 Burma; coll. Dr. F. Stoliczka.

30 Muisraka, Ranigunj and Port Canning; coll. G. Nevill, Esq.

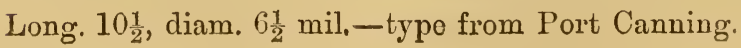


subvar. curta.

30 Silhet; coll. Dr. F. Stoliczka.

Long. $7 \frac{1}{2}$, diam. $5 \frac{1}{4}$ mil.

subvar. rubiginicolor.

20 Zwagabin, nr. Moulmein; coll. Dr. F. Stoliczka.

Long. $7 \frac{1}{2}$, diam. 5 mil.

subvar. attenuata.

30 Botanical Gardens, Calcutta ; coll. G. Nevill, Esq.

30 Pt. Canning; coll. J. Wood-Mason, Esq.

6 Penang; coll. Dr. F. Stoliczka.

Long. $7 \frac{1}{2}$, diam. $4 \frac{1}{2}$ mil.-Botanical Gardens, Calcutta, on shrubs, a quarter of a mile from the river.

\section{Assiminea subconica.}

Optediceros subconicum, Leith, J. As. S. Bombay $V, 1853$, Bombay"animal grey"-long. 4, diam. $2 \frac{1}{4}$ mil.

Mr. Fedden has discovered a single specimen of an allied species, with markedly more subangulate last whorl and less convex spire, subfossil in the Runn of Kutch.

18 Bombay; coll. Rev. S. B. Fairbank and W. T. Blanford, Esq.

$$
\text { var. (? distinct species). }
$$

8 Bombay (in bad condition); coll. Rev. S. B. Fairbank.

2 Kathiawar; coll. F. Fedden, Esq.

\section{Assiminea peaseana.}

Assiminea peaseana, H. Nevill, ms., G. Nevill, J. Asiat. S. Bengal, 1S80, Ceylon.

12 Ceylon (type); coll. H. Nevill, Esq.

\section{Assiminea theobaldiana.}

Assiminea theobaldiana, G. Nevill, J.Asiat. S. Bengal, 1380, Pt. Canning and op. cit. 1881, pl. VII, fig. 4.

30 Port Canning (type); coll. J. Wood-Mason, Esq.

1 Chandipal; coll. G. R Clarke, Esq. 
7. Assiminea, n. sp.

1 Kulungsu (Amoy); coll. R. Hungerford, Esq.

Near Assiminea microsculpta, Nevill.

8. Assiminea, n. sp. (?)

1 Bombay; ex c. Dr. F. Stoliczka.

9. Assiminea japonica.

Assiminea Japonica, von Martens, Berl. Ges. naturl. Fr. 187\%, Japan.

2 Japan; ex Mr. R. Damon (labelled “A. cincta, A. Ad.”).

\section{Assiminea borneensis.}

Amnicola bornecnsis, Issel, Ann. Mus. Civ. Genova VI, 1874, Bintulu-long. $2 \frac{1}{2}$, diam. $1 \frac{2}{3}$ mil.

4. Borneo (typical); ex c. Prof. A. Issel.

\section{Assiminea beddomeana.}

Assiminea beddomeana, G. Nevill, J. Asiat. S. Bengal, 1S80, Pt. Canning and op. cit. 1881, pl. VII, fig. 3 .

50 Port Canning (type) ; coll. J. Wood-Mason, Esq.

11 Cox's Bazar (Chittagong) and Rangoon; coll. H. Raban and R. Hungerford, Esqrs.

\section{Assiminea rotunda.}

Assiminea rotunda, Fairbank, ms., W.T.Blanford, Ann. Mag. XIX, 1S67"on mud between tidesmarks, rather lower down than $A$. cornea and A. marginata, Bombay-long.4, diam. 3 mil., animal grey."

8 Sonmiani, Mekran (Persian Gulf); coll. W. T. Blinford, Esq.

16 Bombay; coll. Rev. S. B. Fairbank and W. T. Blanford, Esq.

\section{Assiminea marginata.}

Optediceros marginatum, Leith, J. As. S. Bombay, V-July 1853, Bombay-

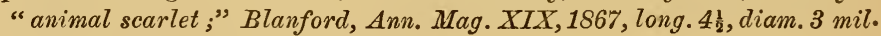
and var. major, long. 6, diam. 4 mil.

14 Bombay; coll. Rev. S. B. Fairbank and W. T. Blanford, Esq.

8 Sonmiani, Persian Gulf; coll. W. T. Blanford, Esq.

The differences between this species and the preceding seem to me scarcely more than varietal. 
14. Assiminea, n. sp.

Beypore; coll. Rev. S. B. Fairbank.

Long. (vix) $2 \frac{1}{4}$, diam. 2 mil.

\section{Assiminea cornea.}

Optediceros corneum, Leith, J. As. S. Bombay, V-1853, Bombay [not A: cornea, Pfr. 1854, Borneo]; fide Blanford 186\%, "long.4, diam. 3 mil.rare."

8 Bombay ; coll. Rev. S. B. Fairbank and W. T. Blanford, Esq.

Leith describes the animal of this shell, as also that of $A$. subconicum, as of a grey colour. That of $A$. marginatum, he states, is scarlet.

\section{Assiminea brevicula.}

Hydrocena brevicula, Pfeiffer, P. Zool.S. 1854, Singapore, long. $8 \frac{1}{3}$, diam. $5 \frac{1}{2}$ mil.; var. = A. miniata, Martens, Ann. Mag. XVII, p. 204, 1866, Singapore, long. 9, diam. 4 mil. ; = A. rubella, Blanford, Ann. Mag. 1867, Ira. wady Delta, long. 5, diam. 4 mil.; var. = Hydrocena marginata, Morelet, J. de C. 1865, Bangkok-long. 6 to 8, diam. 4 to $5 \frac{1}{3}$ mil.; ? = A. rubida, Gould, P. Bost. S. 1859, Loo Choo I.; Nevill, J. A. S. B. 1881, pl. VII, fig. 5 (animal).

12 Singapore ; ex c. India House Museum.

1 Malacca; coll. Dr. F. Stoliczka.

20 Andamans; coll. J. Wood.Mason and F. A. de Roepstorff, Esqrs.

Long. $6 \frac{1}{4}$, diam. 5 mil.

3 Borneo; ex Mr. Damon (c. A. Adams).

50 Irawady Delta; coll. W. T. Blanford, Esq., \&c.

50 Port Canning; coll. J. Wood-Mason and G. Nevill, Esqrs. $(7,5)$.

14. Arakan; coll. Dr. F. Stoliczka.

$8 \mathrm{Cebu}$ (Philippines); coll. R. Hungerford, Esq.

12 Kulungsu (Amoy); coll. R. Hungerford, Esq.

\section{subvar. bibalteata.}

12 Port Canning; coll. G. Nevill, Esq., \&c.

subvar. bicolor.

20 Port Canning; coll. J. Wood-Mason, Esq., \&c.

subvar. flavida.

12 Port Canning; coll. Dr. F. Stoliczka, \&c. 
17. Assiminea paladilhii.

Omphalotropis Paladilhii, Issel, Ann. Mus. Civ. Genova DI, 1874, Sarawak

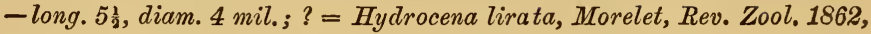
long. $6 \frac{1}{2}$, diam. 4 mil., banks of the Bien.Hoa, Cochin-China.

2 Sarawak (typical); ex c. Prof. A. Issel.

18. Assiminea eliæ.

Assiminea Eliae, Paladithe, Ann. Sci. Nat. (VI), Vol. II, 1875, Rochelle and Coimbra.

20 Bayonne; coll. G. Nevill, Esq.

A true Assiminea and very like the Chinese A. sinensis.

\section{Assiminea latericea.}

Assiminea latericea, H. \& A. Adams, P. Zool. S. 1863, p. 434, Yang-tszekiang \& Peiho.

1 China (typical); ex c. H. Adams, Esq.

20. Assiminea (?) dubia.

Cyclostoma dubium, Pfeiffer, Zeits. Mal.1846, Opara-long. 9, diam. 5 mil.; fide Pease, 1869, species of Assiminea.

4 Opara (typical ?); ex c. Dr. H. Dehrn.

I believe this will prove a species of Omphalotropis, rather than of Assiminea.

1 Japan (?); ex Mr. R. Damon.

Long. $5 \frac{3}{4}$, diam. 4 mil. Despite the difference in size, it agrees exactly with typical figure in the Conch.-Cab. II.

21. Assiminea subcornea, n. sp.

Hydrocena cornea, Pfeiffer, P. Zool. S. 1854, Borneo \& Bashee I.-long. 10, diam. 5 mil. (not Optediceros corneum, Leith, 1853).

1 Japan (?); ex Mr. R. Damon (as “A. cornea, Pfr. coll. Rein.”).

This looks to me a true Assiminea; long. 7, diam. 4 mil.

22. Assiminea (?), sp.

1 Opara; ex c. Dr. H. Dohrn (mixed with Ass.? dubia).

Long. 5, diam, 4 mil. Probably a species of Omphalotropis. 


\section{Assiminea hungerfordiana.}

Assiminea hungerfordiana, G. Nevill, Journ. Asiat. Soc. Bengal, 1880, Rangoon and op. cit. 1881, pl. VII, fig. 2.

1 Rangoon (type); coll. R. Hungerford, Esq.

\section{Assiminea sinensis.}

Assiminea sinensis, G. Nevill, Journ. Asiat. Soc. Bengal, 1880, Hongkong.

3 Estuary of the Canton Rv.; coll. R. Hungerford, Esq.

4. Hongkong (type); coll. R. Hungerford, Esq.

\section{Assiminea woodmasoniana.}

Assiminea woodmasoniana, G. Nevill, J. Asiat. Soc. Bengal, 18s0, Pt. Can. ning, and op. cit. 1881, pl. VII, fig. 1.

30 Port Canning (type); coll. J. Wood-Mason, Esq.

2 Chandipal; coll. G. R. Clarke, Esq.

$$
\text { var. (? dist. sp.) }
$$

1 Bombay; coll. W. T. Blanford, Esq.

\section{Assiminea cincta (?).}

Assiminea cincta, A. Adams, Ann. Mag. VIII, 1861, Estuary of the Peiho.

1 Amoy; coll. R. Hungerford, Esq.

\section{Assiminea dohrniana.}

Assiminea dohrniana, G. Nevill, J. Asiat. S. Bengal, 1S80, Hongkong.

10 Hongkong (type); coll. R. Hungerford, Esq.

1 Kulungsu (Amoy); coll. R. Hungerford, Esq.

\section{Assiminea bifasciata.}

Assiminea bifasciata, G. Nevill, Journ. Asiat. S. Bengal, 18S0, Natal.

12 Port Natal (type); coll. G. Nerill, Esq. $\left(5 \frac{1}{2}, 3 \frac{1}{2}\right)$.

Long. $5 \frac{1}{2}$, diam. $3 \frac{1}{2}$ mil.

\section{Assiminea lutea (?).}

Assiminea lutea, A. Adams, Ann. Mag. 1861, mouth of the Peiho; ? = A debilis, Gould, P. Bost. S. VII, 1859, p. 107, Loo Choo I.

1 Deep Bay (Hongkong); coll. R. Hungerford, Esq. 
var. fasciata.

1 Dsep Bay, Hongkong; coll. R. Hungerford, Esq.

30. Assiminea microsculpta.

Assiminea microsculpta, G. Nevill, Journ. Asiat. S. Bengal, 1880, Pt. Canning, and op. cit. 1881, pl. VII, fig. 5.

50 Port Canning (type); coll. J. Wood-Mason, Esq.

\section{Assiminea templeana.}

Assiminea templeana, G. Nevill, Journ. Asiat. S. Bengal, 1880, Nicobars.

20 Katchall, Nicobar Islands (type); coll. F. A. de Roepstorff, Esq.

\section{Assiminea affinis.}

Assiminea affinis, Mousson [ubi ?].

1 Rockhampton [Queensland]; ex Mus. Godeffroy (as "A. affinis, Mss.").

33. Assiminea ovata.

Hydrocena ovata, Pease, P. Zool. S. 1864, Mangier I.-long. 5, diam. 3 mil. ; fide Pease, 1869, Omphalotropis sp.; fide Cat. Mus. Godef., Assiminea sp.

2 Cook's I. (=Hervey I.); ex Mus. Godeffroy.

\section{Assiminea vitiensis.}

Assiminea vitiensis, Garrett, Am. J. Conch. VII, 1872, Viti I.-long. $4 \frac{1}{2}$ mil.

1 Viti (=Feejee I.) ; ex Mus. Godeffroy.

\section{Assiminea parvula.}

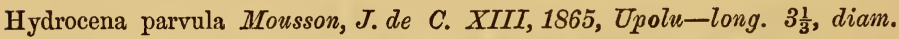
$2 \frac{1}{4}$ mil.; = Hydrocena similis, Baird, Voy. Curacoa; Omphalotropis sp. fide Pease, 1869 ; Assiminea sp., fide Cat. Mus. Godeffroy.

3 Upolu [Samoa]; ex Mus. Godeffroy.

I consider this probably a mere variety (and a very slight one) of Assiminea nitida.

\section{Assiminea moussoni.}

Amnicola moussoni, Issel, Ann. Mus. Civ. Genova, DI, 1874, Bintululong. $2 \frac{1}{2}$, diam. $1 \frac{3}{4}$ mil.

7 Sarawak [typical]; ex c. Prof. A. Issel.

I doubt if this is more than a variety of the preceding. It is a smaller form, with less acuminate spire, slightly less angular at the periphery, with a smaller and more contracted aperture. 
37. Assiminea nitida.

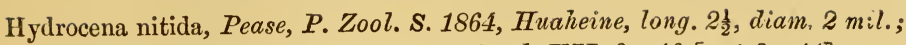
Assiminea sp. fide Pease, J. de C. 1869, pl. VII, fig. 10 [not fig. 11].

3 Pacific I. [typical]; coll. H. Pease, Esq.

30 Ceylon; coll. G. Nevill, Esq.

30 Mauritius; coll. G. Nevill, Esq.

An incised line beneath the suture exists in several of these specimens from Mauritius.

var.

3 Huaheine [Tahiti]; ex Mus. Godeffroy (as "A. nitida").

This is a form more like Assiminea parvula than the preceding typical specimens, the whorls being slightly more convex; it is smaller than either.

var. nana.

16 Mauritius (type var.) ; coll. G. Nevill, Esq.

1 Penang; coll. Dr. F. Stoliczka.

1 Nicobars; coll. J. Wood-Mason, Esq.

38. Assiminea, n. sp.

8 Chandipal (type); coll. G. R. Clarke, Esq.

Very close to the preceding, but with the last whorl relatively much more globose, the spire shorter, like Amn. borneensis, Issel, l. c., but imperforate, \&c.

Long. $1 \frac{1}{2}$ mil.

39. Assiminea, n. sp. (?)

2 Bombay (in bad condition); coll. W. T. Blanford, Esq.

40. Assiminea carinata.

Assiminea carinata, Lea, P. Ac. Philad. VII, 1856, Siam; = Hydrocena fas. ciolata, Morelet, Rev. Zool. 1862, Cochin-China, long. 10, diam. 5 mil. ; fide Martens $=$ Hydrocena fulvida, Pfr., J. de C. 1862, Siam-long. 7 $\frac{1}{2}$, dian. $3 \frac{s}{4}$ mil.; fide Pease $=$ Omphalotropis maculata, Martens, P. Z. S. 1860, Siam, long. 11, diam. 7 mil.; ?= Hydrocena radiata, Pfr., P. Z. S.

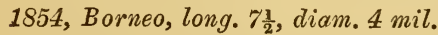

3 Saigon (typical $A$. fasciolata); ex c. A. Morelet, Esq.

1 Sarawak; ex c. Prof. A. Issel (as "A. carinata").

2 Borneo; ex Mr. R. Damon (ex c. A. Adams). 
These last were sent to me as "Assim. radiuta, Pfr." If this identification is correct, Lea's name will have to yield priority to Pfeiffer's.

$$
\text { var. (? dist. sp.) }
$$

1 Zwagabin, nr. Moulmein (sp. juv.); coll. Dr. Stoliczka.

Unfortunately not adult; there is a distinct keel round the umbilicus, also two impressed lines below the suture.

\section{Assiminea littorina.}

Helix littorina, Delle Chiaje, Mém. An. sans Tert. Napoli III, 1828; sect. Paludinella.

3 England; ex Mr. R. Damon.

\section{Assiminea sicana.}

Assiminea sicana, Brugnone, Monterosato, Enum. Conch. Medit. 1576, for A. littorina, Delle Chiaje, var. sicana, Brugnone, Misc. II, Pal. 1876, fig. 15.

5 Algiers; coll. P. Joly, Esq.

43. Assiminea californica.

Assiminea californica, Cooper ( $u b i$ ?).

4. San Francisco; ex "Linnæa."

44. Assiminea n. sp.

1 Andamans; coll. F. A. de Roepstorff, Esq.

Closely allied to A. woodmasoniana, Nevill.

45. Assiminea n. sp.

1 Andamans (spec. juv.); coll. F. A, de Roepstorff, Esq.

Allied to $A$. miscrosculpta, Nevill.

46. Assiminea turbinata.

Hydrocena turbinata, Gassies, Act. Soc. Lin. Bord. 1879, Nouméa et Ile Nou-long. 3 to $3 \frac{1}{2}$, diam. 2 to $2 \frac{1}{2}$ mil.

10 New Caledonia; ex c. P. Bouvier and C. F. Ancey, Esqrs.

\section{Assiminea pygmaea.}

Hydrocena pygmaea, Gassies, J. de C. 1865, Iles Art et Nou-long. 2, diam. $1 \frac{1}{2}$ mil.

3 New Caledonia; ex c. P. Bouvier, Esq. 


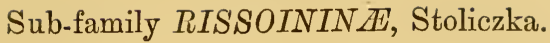

\section{Genus RISSOINA, Orbigny.}

Voy. Amér. Mérid. V, p. 394, 1842 [as Sub-Genus of Rissoa], unique type R. inca, Orb.; sect. B.=Rissolina, Gould, P. Bost. S. 1861, type R. plicatula, Gld.; sect. C. = Schwartziella, Nevill, type R. bryerea, Mtg.; sect. $\boldsymbol{D} \equiv$ Phosinella, Mörch, type R. pulchra, C. B. Adams; sect. E. = Zebinella, Mörch, type R. decussata, Mtg.; sect. $F$. = Moerchiella, Nevill, type $R$. gigantea, Deshayes; sect. G.=Zebina, H. \& A. Adams, type R. coronata, Recluz; sect. H. = Pyramidelloides, Nevill, type R. miranda, A. Adams.

\section{Section A.-TypicaL,}

Rissoina costulata, Pease, Am. J. Con., 1868, Paumotus, long 5, diam. 2 m., belongs to the typical section as does R. incerta, Souverbie, from New Caledonia, and R. terebra, Garrett, from the Pacific Islands.

\section{Rissoina ambigua.}

Pyramidella ambigua, Gould, P. Bost, S. III, 1849, Clermont Tonnerre, long. $\frac{1}{4}$, diam. $\frac{1}{10}$ poll.-[emend. Otia Con. p. 245, as Rissoina]; $=$ R. multicostata, Garrett, P. Calif. Acad. 1857 (not of C. B. Ad. $1850)=R$. costulifera, Pease, J. de C. 1862, p. 392, Sandwich Islands ; $=R$. pusilla, schwartz, pl. IV, fig. 29 [not of Brocchi]; $=R$. subconcinna, Souverbie, J. de.C. 18 , New Caledonia, long. 6 to 81, diam. $2 \frac{1}{4}$ mil.

The form originally described by Gould was evidently young, hence the many errors that have arisen. The R. pusilla of Brocchi is scarcely separable! Schwartz's pl. III, fig. 27, "R. ambigua," seems to be something different.

100 Ceylon, Mauritius, Andamans, Arakan, Penang, Annesley Bay and Roweiah [Red Sea], Seychelles, Bourbon and Aden; coll. W. T. Blanford, F. Stoliczka, J. B. Baxter, J. Wood-Mason, and G. Nevill, Esqrs.

10 Upolu and Sandwich I.; coll. H. Pease, Esq., and ex Mus. Goleffroy. 3 New Caledonia; ex c. P. Bouvier, Esq. 
subvar. cincta.

Rissoina cincta, Dunker, Cat. Mus. Godef.IV, 1869, Upolu; op. cit. V, as var. of R. pusilla.

13. Mauritius, Cejlon, Arakan and Andamans; coll. W. T. Blanford, J. B. Baxter, J. Wood-Mason, and G. Nevill, Esqrs.

var. perpusilla, nov.

Long $3 \frac{1}{10}$, diam. $1 \frac{1}{3}$ mil.

26 Mauritins [type var.]; coll. G. Nevill, Esq.

3 Bombay and Henjam [Persia]; coll. W. T. Blanford, Esq.

var. myosoroides.

R. myosoroides, Recluz, fide Schwartz, pl. IV, fig. 30, Mauritius.

30 Arakan, Mauritins, Ceylon, Natal, Andamans, and Singapore; coll. F. Stoliczka, J. Wood-Mason, and G. Nevill, Esqrs.

\section{Rissoina pusilla.}

Turbo pusillus, Brocchi, Conch. foss. II, 1814.

3 Steinabrun [fossil]; coll. Herr G. Schwartz von Mohrenstern.

The callosity of the outer lip and the twist of the columella are much more developed than in the preceding species; it is, however, most closely allied and perhaps scarcely specifically separable!

\section{Rissoina woodmasoniana, n. sp.}

1 Andamans [type]; coll. J. Wood-Mason, Esq.

A single living specimen of this remarkable new form was dredged by Mr. Wood-Mason, if I remember rightly, in deep water. Nine whorls, cylindrical and "needle-like," the apical three smooth and colourless, the others longitudinally closely ribbed, ribs thick, flexuous and irregular; of a uniform brown colour throughout; columella straight, aperture contracted, subangulate at base, not notched, resembling that of species of Zippora.

Long. 3, diam. $\frac{3}{4}$ mil. 


\section{Rissoina indica, n. sp.}

1 Paumben Straits, South India [type]; coll. F. Armstrong, Esq.

Another very beautiful, shining, "Mangelia-like" form, dredged alive in 35 fathoms; it is not unlike $R$. terebra, Garrett, Pl. 2, fig. 11, of the Proc. Acad. Philad., and also resembles $R$. incerta, Souverbie! It has eight perfectly "planulate" whorls, the apical one smooth, the others with perfectly perpendicular, regular, very massive ribs, otherwise smooth; columella and interior of aperture stained brown, scarcely notched at base, outer margin white and remarkably thickened.

Long. 5, diam. $1 \frac{5}{6}$ mil.

\section{Rissoina subdebilis.}

Rissoina subdebilis, Nevill ms., Weinkauff, Conch.-Cab. II, 1Ss1, pl. 15 B, fig 9, Mauritius.

20 Mauritius (type); coll. G. Nevill, Esq.

May possibly prove to be a large, cylindrical, subangulate variety of $R$. debilis, Garrett, P. Acad. Philad.1873, pl. 2, fig. 9.

Long. 6, diam. $2 \frac{1}{2}$ mil.

var. perlanceolata [? dist. sp.]

1 Mauritius (type var.); coll. G. Nevill, Esq.

A well-marked form with ten whorls.

\section{Rissoina, n. sp.}

5 Kesm (Persian G.) ; coll. W. T. Blanford, Esq.

Somewhat resembles the fossil $R$. grateloupi, Orbígny.

Long. $3 \frac{3}{4}$, diam. $1 \frac{1}{2}$ mil.

? var. [? dist. sp.]

1 Seychelles; coll. G. Nevill, Esq.

Long. 4, diam. $1 \frac{3}{4}$ mil.

? var. [? dist. sp.]

2 New Caledonia; ex c. P. Bouvier, Esq. 


\section{Rissoina micans.}

Rissoina micans, A. Adams, P. Zool. S. 1851, Mindanao; Schwartz, fig. 8 (typical spec,), long. 4.7 , diam.1.7 mil.

100 Tumb I., Kesm and Mutef (Persian G.) ; coll. W. T. Blanford, Esq.

var. perstriatula, nov.

1 Tumb I. ; coll. W. T. Blanford, Esq.

\section{Rissoina, n. sp.}

1 Mauritius ; coll. G. Nevill, Esq.

Near the fossil $R$. basteroti.

\section{Rissoina elegans.}

Rissoa elegans, d'Orbigny, Conch. foss. 153S, Dax.

var. [? dist. sp.]

1 Steinabrunn (fossil); coll. Herr Schwartz von Mohrenstern.

\section{Rissoina variegata.}

Rissoina variegata, A. Adams and Angas, P. Zool. S. 1867, Port Jackson.

2 Australia; ex c. Dr. F. Stoliczka.

\section{Rissoina bruguierei.}

Rissoa bruguierei, Payrandeau, Cat. Moll. Corse, $18: 6$.

30 Algiers, Rhodes, \&c; coll. P. Joly, Esq. \&c.

\section{Rissoina monilis.}

Rissoina monilis, A. Adams, P. Zool. S. 1851, Philippines.

This species is recorded by von Martens from Mauritius, but I have not been able to identify it with any of the species I have met with. Schwartz von Mohrenstern gives the measurements of a typical specimen as-long. $4: 9$, diam. 3 mil. 
13. Rissoina nevilliana.

Rissoina nevilliana, Weinkauff, Conch.-Cab.II, 1881,pl.15 D, fig. 2, Kowloon; Nevill, J. A. S. B., 1881, long. $13 \frac{1}{2}$ diam. $4 \frac{1}{2}$ mil.

1 Kowloon, opposite Hongkong [type]; coll. R. Hungerford, Esq.

\section{Section B.-Rissolina, Gould.}

P. Bost. S. 1881, type R. plicatula, Gld.; Group I, Section B., Schwartz l. c., page 38 .

\section{Rissoina minuta.}

Rissoina minuta, G. Nevill, J. A. S. B., 1874, Andamans, long. 2⿺辶2 diam. 1 mil.

2 Andamans [type]; coll. G. Nevill Esq.

\section{Rissoina funiculata.}

Rissoina funiculata, Souverbie, Journ. Conch. XIV, 1866, New Caledonia.

20 Ceylon and Andamans; coll. J. Wood-Mason, J. B. Baxter, and G. Nevill, Esqrs.

\section{Rissoina weinkauffiana.}

Rissoina weinkauffiana, G. Nevill, J. A. S. B. 1S81, Andamans, and Conch.Cab. II, 1881, pl. 15 B. fig. 7.

14 Andamans [type]; coll. J. Wood-Mason and G. Nevill; Esqrs.

\section{Rissoina burdigalensis.}

Rissoina burdigalensis, d'Orbigny, Prod.|III, p. 30, 1852 [fossil], Bordeaux; fide Schwartz, recent from Mauritius.

1 Steinabrunn [fossil]; coll. Herr Schwartz von Mobrenstern.

\section{Rissoina subangulata.}

Rissoina subangulata, C. B. Adams, Contr. Conch.1860, p. 112, Jamaica.

8 W. Indies ; ex c. J. Wood-Mason, Esq.

\section{Rissoina n. $\mathrm{sp}$.}

Anfr. 6 ; long. $2 \frac{1}{2}$ mil.

1 Mauritius; coll. G. Nevill, Esq. 


\section{Rissoina plicata.}

Rissoina plicata, A. Adams, P. Zool. S. 1851, I. Masbate; Schwartz, l. c. fig. 21 [typical spec.], long. 5*6, diam. 2.5 mil.; var. $=R$. scalariana, $A$. Ad. and R. bertheloti, Audouin.

50 Andamans, Arakan, Kharg, Mauritius, Bourbon, Seychelles; coll. W. T. Blanford and G. Nevill, Esqrs.

10 Ceylon; coll. G. Nevill, Esq. [6,2 $\frac{3}{4}$-apex perfect].

These Ceylon specimens approximate very closely to var. R. bertheloti.

1 Viti-Levu ; ex Mus. Godeffroy [as " R. firmata, C. B. Ad."]

A true, typical R. plicata, and not in the least like the W. Indian $R$. firmata.

2 New Caledonia; ex c. P. Bouvier, Esq.

subvar. diplostoma.

1 Ccylon; coll. G. Nevill, Esq.

21. Rissoina scalariana.

Rissoina scalariana, A. Adams, P. Zool. S. 1861, Philippines ; fide Schwartz, long. $8 \frac{1}{2}$, diam. $3 \frac{1}{3}$ mil.

1 Roweiah [Red Sea]; coll. J. B. Baxter Esq.

4 Mauritius, and Kharg [Yersian G.]; coll. W. T. Blanford and G. Nevill, Esqrs.

Possibly better classed as a variety of $R$. plicata.

\section{Rissoina bertheloti.}

Rissoina bertheloti, Audouin, Descr. de l'Egypte, 1S26, $p l . I V$, fig. 2.

Long. $8 \frac{1}{4}$, diam. $3 \frac{1}{2}$ mil.

1 Kesm ; coll. W. T. Blanford, Esq.

20 Roweiah and Aden; coll. J. B. Baxter and G. Nevill, Esqrs.

subvar. minor.

Long. 5, diam. $2 \frac{1}{10}$ mil.

3 Roweiah, Red Sea ; coll. J. B. Baxter, Esq. 


\section{Rissoina canaliculata.}

Rissoina canaliculata, Schwartz, K. Ak. Wissens. Wien, XIX, 1S60, Philippines, long. 10· 6 , diam. 4.1 mil.

1 New Caledonia ; ex c. P. Bouvier, Esq.

30 Ceylon; coll. J. B. Baxter and G. Nevill, Esqrs. $\left[10 \frac{1}{4}, 4 \frac{1}{2}\right]$.

A crow carried off a tube full of my best Ceylon specimens !

3 Andamans; coll. Capt. R. C. Temple and Colonel Berkely [11, 4, $\left.\frac{1}{3}\right]$.

8 Ivatal ; coll. G. Nevill, Esq. $\left[7 \frac{1}{4}, 2 \frac{3}{4}\right]$.

A very pretty laneeolate form from Natal, with acute apex, and more turriculate, that is with less convex whorls, than in the Ceylon one, and in this exactly resembling the typical figure.

subvar. minor [? dist. sp.]

1 Mauritius; coll. G. Nevill, Esq.

In such bad condition that it is impossible to be sure of the absence of spiral striation.

\section{Rissoina monilifera, n. sp.}

Long. 8, diam. 3 mil.

A very handsome and distinct species, resembling the fossil R. lamellosa, Desmoulins, Schwartz, Pl. II, fig. 14, from Dax.

1 Japan; ex Mr. Damon (coll. A. Adams; labelled “ $R$. monilifera").

\section{Rissoina plicatula.}

Rissoina plicatula, Gould, P. Bost. S. VII, 1861 (Febr.), Bonin and Loo Choo I.-long. 6, diam. 2 mil.

A more convexly rounded sp. than $R$. plicata, with more numerous and less perpendicular ribbing.

31 Roweiah, Aden, and Annesley Bay; coll. J. B. Baxter, and W. T. Blanford, Esqrs. $\left[4 \frac{4}{5}, 2\right]$.

8 Ceylon; coll. G. Nevill, Esq. [6 to 7, $2 \frac{1}{2}$ to 3$]$.

Though in poor condition the interstitional, spiral striation is distinctly traceable, under a lens. 
1 Mauritius ; coll. G. Nevill, Esq.

12 Kesm [Persian G.]; coll. W. T. Blanford, Esq.

Long. $6 \frac{1}{4}$, diam. $2 \frac{4}{5}$.

var.? [dist. sp. ?]

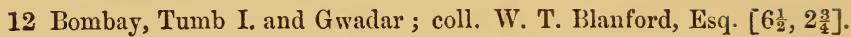

I can see no trace of spiral striation, but the specimens are all in most wretched condition.

They may be a var. of $R$. bertholeti, but the ribs are irregularly flexous instead of regularly perpendicular, and a little more numerous; it is also a more attenuate shell.

\section{Rissoina turricula.}

Rissoina turricula, Pease, P. Zool. S., 1860, Sandwich I.

1 Sandwich Isl. [typical] ; coll. W. Pease, Esq. $\left[3 \frac{1}{3}, 1 \frac{1}{2}\right]$.

2 Mauritius; coll. G. Nevill, Esq.

1 Roweiah, Red Sea; coll. J. B. Baxter, Esq.

var. cernica, Nevill.

Long. $2 \frac{1}{2}$, diam. 1 mil.

10 Mauritius; coll. G. Nevill, Esq.

var. ceylonica, Nevill.

2 Galle [type var.]; coll. J. B. Baxter, Esq.

Long. 3, diam. 1 mil.

Whorls $6 \frac{1}{2}$, two apical ones smooth, the others somewhat convexly turriculate, with flexuous, rather distant, well-developed, longitudinal ribs, the last of all with a basal, subgranulose, transverse keel, with two transverse, fine, but distinct, striæ above it ; basal emargination of aperture well marked; peristome callously thickened; throughout, almost microscopically, transversely striate. 


\section{Rissoina obeliscus.}

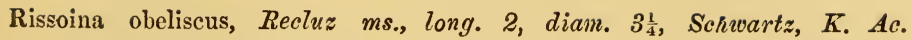
Wissens. Wien, XIX, 1860, fig. 15, Mauritius, long. 4, 5, diam. 2 mil.also from Sydney, l. c.; $=R$. schwartziana, Dkr., Cat. M. G. IV, 1869, Upolu.

18 Andamans, Arakan, Ceylon, Mauritius, Annesley Bay; coll. W. T. Blanford, J. Wood-Mason, and G. Nevill, Esqrs.

2 Upolu; ex Mus. Godeffroy.

subvar. nana.

2 Galle; coll. J. B. Baxter, Esq.

\section{Rissoina subfuniculata.}

Rissoina subfuniculata, G. Nevill, Weinkauff, Conch.-Cab.II, 1S81, Persian Gulf, \&c., Pl.15 B, fig. 8-long. 4, diam. $1 \frac{1}{4}$ mil.

20 Kesm, Gwadar [type] and Tumb I. ; coll. W. T. Blanford, Esq.

1 Andamans; coll. G. Nevill, Esq.

Long. $4 \frac{1}{4}$, diam. $1 \frac{3}{4}$ mil.

var. fortis.

3 Andamans [type var.]; coll. G. Nevill, Esq.

Long. 5, diam. 2 mil.

var. validicostata [? dist. sp.]

1 Singapore [type var.]; coll. G. Nevill, Esq.

3 Arakan; coll. W. T. Blanford, Esq.

Long. 3, diam. $1 \frac{1}{2}$ mil.

29. Rissoina, n. sp.

1 Mauritius; coll. G. Nevill, Esq.

30. Rissoina, n. sp.

4. Kesm, Persian Gulf; coll. W. T. Blanford, Esð. 


\section{Section C.-Schwartzielda, Nevill.}

Schwartz, l. c., page 38, type R. bryerea, Montagu, as Group II, section $B$.

\section{Rissoina chesneli.}

Rissoina chesneli, Michaud, Descr. esp.nouv., 1832, W. Indies; fide Schwartz, long. 3. 6, diam. 1. 4 mil.-W. Indies and Mauritius.

2 W. Indies; ex c. J. Wood.Mason, Esq.

\section{Rissoina baxteriana.}

Rissoina baxteriana, G. Nevill, J. A. S. B., 1851, Roweiah and Conch.• Cab. II, 18S1, pl. . fig.

4. Roweiah, Red Sea [type] ; coll. J. B. Baxter, Esq. $\left[3,1 \frac{1}{4}\right]$.

\section{Rissoina bryerea.}

Turbo bryereus, Montagu, Test. Brit. 1803; fide Schwartz, from Cuba and Maurilius.

20 W. Indies; ex c. J. Wood-Mason and G. Nevill, Esqrs.

\section{Rissoina pseudo-bryerea.}

Rissoina pseudo-bryerea, G. Nevill, J. A. S. B., 1881, Roweiah ; ? R. bryerea, Schwartz, fig. 36A. from Mauritius.

2 Roweiah, Red Sea (type); coll. J. B. Baxter, Esq.

Anfr. $6 \frac{2}{2}$, long. $5 \frac{1}{2}$, diam. $2 \frac{1}{4}$ mil.-More turriculate, with last whorl more contracted than in the above quoted fig.; the ribs broader, more perpendicular, aperture smaller. Quite distinct from the $W$. Indian species.

\section{Rissoina triticea.}

Rissoina triticea, Pease, P. Zool. S. 1860 , South Sea Islands; =R. scalariformis, Auct. [not of C. B. Adams];=R. orientalis, Nevill, J. A. S. B., 18؟1, Ceylon [type]; vars. ?=R. exigua, Dunker, Cat. Mus. Godeff. IV and Conch.-Cab. II, pl. 15C, fig. 3, Upolu; var.?=R. rissoi, Audouin, Expl. Savigny, Desc. de l'Egypte, 1826.

56 Mauritius, Bourbon, Ceylon, and Andamans; coll. J. Wood-Mason and G. Nevill, Esqrs.

108 Roweiah, Annesley Bay, and Aden; coll. W. T. Blanford and J. B. Baxter, Esqrs. 
Probably priority will have to be given to M. Audouin's name.

var. microstoma [an R. microstoma, sp. uov. ?]

Long. $4 \frac{1}{2}$, diam. 2 mil.

1 Omm [Persian G.] ; coll. W. T. Blanford, Esq.

36. Rissoina scalariformis.

Rissoina scalariformis, C. B. Adams, Cat. Panama Shells, 1852, long:3-3 lat. 1.27; fide Schwartz, Panama and Mauritius, also probably smaller var. Red Sea.

var?

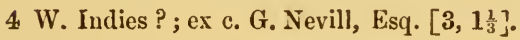

37. Rissoina mainwaringiana, n. sp.

10 Persian Gulf [type]; ex c. Colonel G. B. Mainwaring.

38. Rissoina, n. sp.

Long. 3, diam. $1 \frac{1}{3}$ mil. This cannot be any of Gould's species; it resembles the W. Indian R. firmata, C. B. Adams.

1 Hongkong; coll. R. Hungerford, Esq. ("very rare").

\section{Section D.-Phosinelua, Mörch.}

Mal. Blat. XXIII, 1876, type R. pulchra, C. B. Ad.; = Group IV, Schwartz, l. c., page 39.

There is a $R$. balteata, Pease, from Hawaii, long. 4, diam. $1 \frac{1}{4}$ mil.-perhaps $=R$. exasperata, Souverbie?

39. Rissoin cancellata.

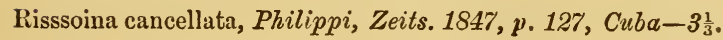

var. pulchra.

Rissoina pulchra, C. B. Adams, Contr. Conch. p. 114, Jamaica; fide Mörch, a distinct species.

4 W. Indies; ex c. J. Wood-Mason, Esq. 


\section{Rissoina media.}

Rissoina media, Schwartz von Mohrenstern, Denks. K. Ak. Wissens. Wien, $X I X, 1860$, Ceylon and Java, also Nicobars-long. 4-7, diam. 2 mil.

100 Ceylon $\left[4 \frac{3}{4}, 2\right.$ mil. $]$, Arakan, Andamans, and Aden; coll, Dr. Sto. liczka, J. Wood-Mason and G. Nevill, Esqrs.

var.?

20 Mauritius; coll. G. Nevill, Esq.

1 Seychelles; coll. G. Nevill, Esq. $\left[3 \frac{1}{4}, 1 \frac{1}{4}\right]$.

\section{Rissoina, n, sp.}

Long. $4 \frac{1}{8}$, diam. $1 \frac{1}{2}$ mil.

3 Tumb I. [Persian G.]; coll. W. T. Blanford, Esq.

\section{Rissoina samœnsis.}

Rissoina samœnsis, Dunker, Cat. Mus. Godeffroy, 1874, Upolu ; and Conch.-

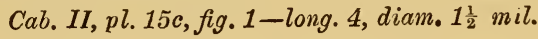

1 Upolu [typical]; ex Mus. Godeffroy.

\section{Rissoina exasperata.}

Rissoina exasperata, Souverbie, J. de C, XIV, 1866, N. Caledonia, anfr. 8 to 9 -long. $3 \frac{1}{2}$ to $4 \frac{1}{2}$, d. $1 \frac{1}{4}$ to $1 \frac{3}{4}$ mil.

1 South Sea Islands; ex Mus. Godeffroy.

1 Singapore; coll. Dr. Stoliczka.

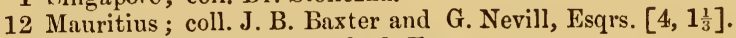

1 Arakan; coll. W. T. Blanford, Esq.

5 N. Caledonia; ex c. P. Bouvier, Esq.

\section{Rissoina seguenziana.}

Rissoina seguenziana, Issel, Malac. Mar. Rosso, 1869, and Descr. de l'Egypte, Pl. IV, fig. 3, G. Suez-anfr. 9 to 10; long. 5, diam. $1 \frac{3}{5}$ mil.

15 Kharg (4, 1 $\left.\frac{3}{5}\right)$, Tumb I. and Bombay ; coll. W. T. Blanford, Esq.

32 Mauritius $(5,2)$, Aden, and Seychelles; coll. G. Nievill, Esq.

20 Roweiah $\left(4 \frac{1}{2}, 1 \frac{3}{4}\right)$; coll. J. B. Baxter, Esq.

$$
\text { var. parvula, nov. }
$$

Long. $3 \frac{1}{2}$, diam. $1 \frac{1}{3}$ mil.

20 Annesley Bay; coll. W. T. Blanford, Esq. 
var. [? dist. sp.]

2 Roweiah, Red Sea; coll. J. B. Baxter, Esq.

var. [?]

1 Andamans; coll. G. Nevill, Esq.

2 Nicobars; coll. Dr. F. Stoliczka.

45. Rissoina, n. sp.

30 Tumb I., Gwadar, \&c. (42 fths.) ; coll. W. T. Blanford, Esq.

\section{Rissoina strigillata [?].}

Rissoina strigillata, Gould, P. Bost. S. 1861, Loo Choo I., axis 5, diam. $2 \cdot 20$ mil.; Conch.-Cab. LI, pl. $15 \mathrm{~B}, \mathrm{fig.4}$, Hong-Kong.

3 Hong-Kong; coll. R. Hungerford, Esq. [4, 1 $\left.\frac{3}{t}\right]$.

"Under stones, at very low water."

47. Rissoina, n. sp.

Long. $2 \frac{1}{6}$, diam. $\frac{3}{4}$ mil.

20 Mauritius and Andamans; coll. G. Nevill, Essq.

48. Rissoina, n. sp.

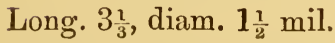

2 Pellang and Andamans; coll. Dr. F. Stoliczka.

1 Andamans; coll. Captain R. C. Temple.

Appears to be a new species near $R$. exusperata, Souv.

49. Rissoina deshagesi.

Rissoina deshayesi, Schwartz von Mohrenstern, Denks. K. Ak. Wissens. Wien, XIX, 1860, MLindanao, long. 9*4, diam. 3.3 mil.

var. minor.

1 Andamans ; coll. J. Wood-Mason, Esq. $\left[6 \frac{1}{2}, 2 \frac{3}{4} \bar{j}\right.$.

$1 \mathrm{Cebu}$; coll. R. Hungerford, Esc. $\left[6_{9}^{3} 2_{\frac{3}{4}}\right]^{2}$ 
The figured type seems to me that of a not quite adult specimen.

\section{Rissoina hungerfordiana.}

Rissoina hungerfordiana, Nevill, Weinkauff, Conch.-Cab. II, 1881, pl. 15 A,

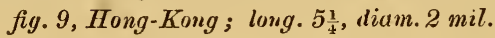

1 Hong-Kong [type]; coll. R. Hungerford, Esq.

51. Rissoina, n. sp.

1 Gwadar; coll. W. T. Blinford, Esq.

52. Rissoina, n. sp.

4. Kesh [Persian Gulf]; coll. W. T. Blanford, Esq.

\section{Rissoina erythræa.}

Rissoina erythræa, Philippi, Zeits. Mal.1851, p. 93, Aden, long. 3.3, diam. $1 \cdot 2$ mil.; fide Schwartz, from Red Sea, Steward's I., Sandwich I. and Mauritius ; fide Mus. Godef. Cat. $V=B$. cerithiiformis, Dkr.

60 Nicobars, Mauritins $\left[4 \frac{1}{6}, 1 \frac{1}{2}\right]$, Andamans $\left[3 \frac{1}{2}, 1 \frac{1}{6}\right]$, Annesley Bay, Roweiah, and Ceylon; coll. W. T. Blanford, G. Nevill, J. WoodMasoll, and J. B. Baxter, Esqrs.

3 Upolu; ex c. Mus. Godeffroy.

\section{Rissoina blanfordiana.}

Rissoina bliufordiana, G. Nevill, J.A.S. B. Sept. 1S81, pl. VI, fig. 16, Zoulla; l.ng. 9 $\frac{1}{2}$, diam. $3 \frac{3}{4}$ m.; =R. Jickelii, Weinkauff, Conch.-Cab.II, Dec. 1S81, Massaua.

1 Zoulla, Red Sen [type R. blanfordiana]; coll. W. T. Blanford, Esq.

1 Massaua [typical R. jickelii]; ex c. Dr. H. C. Weinkauff.

Rissoina jiclielii is the more perfect and adult form of the somewhat "depauperate" specimen from which I described $l$. blanfordiana. Pearl-like nodules on the points of intersection.

\section{Rissoina clathrata.}

Rissoina clatlurata, A. Adams, R. Zuol. S.1S51, Bohol; fide Schwarlz, lony. 12, diam. 45 mil., also from Austrulia; Suez fict Ilc.Andrew. 
40 Aden $\left[11 \frac{1}{2}, 4 \frac{3}{4}\right]$, Singapore [decollate, of 5 whorls-10, $\left.4 \frac{1}{2}\right]$; Andamans [not decollate, 9 whorls $\left.-9 \frac{1}{4}, 3 \frac{1}{4}\right]$, Penang $\left[9 \frac{1}{2}, 4\right]$, Kesm, Gwadar, and Annesley Bay; coll. W. T. Blanford, F. stoliczka, J. Wood. Mason and G. Nevill, Esqrs.

\section{Rissoina, n. sp.}

1 Andamans; coll. G. Nevill, Esq.

Near R. nodicincta, A. Adams.-Anfr. 6 to 7.-Long. 6, diam. $2 \frac{1}{2}$ mil.

\section{Section E.-Zerinella, Mörch.}

Mal. Blät. XXIII, 1876, type R. decussata, Mtg.; = Group III, Schwartz, l.c., page 39.

\section{Rissoina decussata.}

Montagu, Test. Brit. 1503, p. 399 ; fide Schwartz-long. 7·3, diam. 3 mil.; = R. ulata, Menke, Syn. 1830, Hab.?

12 W. Indies; ex c. J. Wood-Mason, Esq.

var. emaciata.

8 Steinabrunn [fossil]; coll. Herr Schwartz von Molirenstern $\left[7 \frac{3}{4}, 3\right]$.

\section{Rissoina obsoleta.}

Melania obsoleta, Partsch,ms., Hörnes, Verz. Foss. Tert. Wien, 184 .

1 Steinabrunn [fossil]; coll, Herr Schwartz von Mohrenstern.

\section{Rissoina adamsiana.}

Rissoina adamsiana, Weinkauff, Conch.-Cab. II., 1881,pl.15 A, fig. 4, Japan. 2 Japan [type]; ex Mr. R. Damon.

From the collection of the late A. Adams, Esq., and labelled "R. strigillata, A. Adams;" it cannot be Gould's species of that name.

\section{Rissoina evanida.}

Rissoina evanida, G. Nevill, Jour. Asiat. Soc. Benyal, 1874, Andamans, long. $2 \frac{1}{2}$, diam. 1 mil.

3 Andamans [type]; coll. G. Nevill, Esq. 


\section{Rissoina mohrensterni.}

Rissoina mohrensterni, Deshayes, Moll. Reunion, 1863, long. 4, diam. 2 mil.; $=R$. schmeltziana, Dkr.from Upolu.

20 Mauritius, Aden, Andamans, and Seychelles; coll. G. Nevill, Esq.

2 Upolu; ex Mus. Godeffroy [as R. schmeltziana, Dkr.]

Very variable in size, long. $5 \frac{3}{4}$, diam. $2 \frac{1}{2}$ mil.-another $4 \frac{1}{2}, 2-$ a third $3 \frac{1}{4}, 1 \frac{1}{3}$, all from Mauritius! Can this be the $R$. tenuistriata of Pease from Paumotu;-long. 9, diam. 4 mil ? I should suppose this can scarcely be the case.

\section{Rissoina andamanica.}

Rissoina andamanica, Theinkauff, Conch..Cab. II, 1881, pl. 15B, fig. 6, Andamans-long. 8, diam. $3 \frac{1}{2}$ mil.

10 Andamans [type]; coll. J. Wood-Mason and G. Nevill, Esqrs.

Long. 7, diam. 3 mil.-another $5 \frac{3}{4}, 2 \frac{1}{2}$ mil.

\section{Rissoina concinna.}

Rissoina concinna, A. Adams, P. Zool. \$. 1851, Philippines, long. 5.3, diam. 2 mil.

Prof. von Martens has recorded the species from Mauritius. I have not been able to identify it with any of the Museum specimens.

$$
\text { Section F.-Mørchielda, Nevill. }
$$

Type R. gigautea, Desh. ; = Group $V$, Schwartz, l. c.

This is the Group V of Schwartz, 1. c., page 39.

\section{Rissoina gigantea.}

Rissoa gigantea, Deshayes, Traité élem. Conch., 1848, fiy. 18, 20 [sine descr.] ; = R. cumingi, H. and A. Adams, Gen. I, 1854, Ticao.

1 Andamans ; coll. Mirjor Rogers [24 $\frac{1}{2}, 10 \frac{1}{2}$, slightly decollate]. 


\section{Rissoina striata.}

Rissoa striata, Quoy, Voy. Astrol. 1836, Tanikoro;=R. grandis, Philippi,

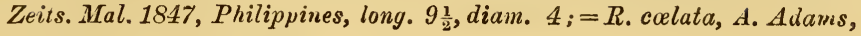
P. Zool. S., 1851, Siquijor.

7 Andamans; coll. Capt. R. C. Temple and J. IVood-Mason, Esq. $\left[19 \frac{1}{2}, 8 \frac{1}{2}\right]$.

1 Philippines; ex c. G. Nevill, Esq.

\section{Rissoina antoni.}

Rissoina antoni, Schwartz von Mohrenstern, Denks. K. Ak. Wissens. Wirn, $X I X, 1860$, Java, long. 7.8, diam. 3 mil. and smaller, more slender var. also from Java, paye 99.

var. pusilla, nov.

Long. 5, diam. $2 \frac{1}{10}$.

1 Locality ?; ex c. G. Nevill, Esq.

\section{Rissoina lamberti.}

Rissoina lamberti, Souverbie, J. de C. 1S70, N. Caledonia, long. 11, diam. 4,

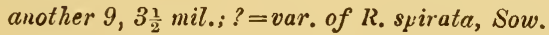

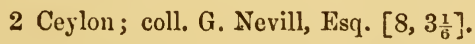

Herr Schwartz considered these specimens, which are in bad condition, as a variety of his $R$. antoni.

1 New Caledonia; ex c. P. Bouvier, Esq.

Unfortunately in bad condition; it may prove a var. of $R$. orbignyana.

\section{Rissoina insignis.}

Rissoina insignis, A. Adams and Reeve, Voy. Samarang, 1S50, China; Schwarlz, l. c., long. 9, diam. 4.5 mil.

var. turrita.

Rissoina turrita, Garretl, P. Ac. Philud. 1873, pl. II, f. 12, Sociely I.long. 7 mil. 
2 Mauritius; coll. G. Nevill, Esq., and Surgeon-Major Power [ $8 \frac{1}{2}$, $\left.4 \frac{3}{4}\right]$.

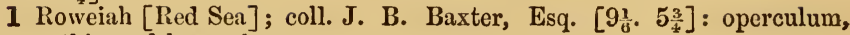
thin and horny-brown.

This variety principally differs from Schwartz's figure in the absence on the antepenultimate whorl of the longitudinal furrows; the characteristic denticulations within the outer lip are well marked in the Mauritian specimens, they are unaccountably wanting in Garrett's magnified figure, though "crenulations within the peristome " are duly recorded in his description! The Red Sea form is considerably stouter and broader than any of the other known ones, as can be seen from the above recorded measurements.

\section{Rissoina japonica.}

Rissoina japonica, Weinkauff, Conch.-Cab. II, 1881, pl. 15A., fig. 1, Japan, ling. $3 \frac{1}{2}$, diam. $1 \frac{1}{4}$ mil.

3 Japan [type]; ex Mr. R. Damon.

Long. 3, diam. $1 \frac{2}{10}$ mil.; from the collection of the late A. Adams, Esq., and labelled "R. pulchella, A. Adams."

\section{Rissoina montrouzieri.}

Rissoina montrouzieri, Souverbie, J. de C., 1862, N. Caledonia, long. $15 \frac{1}{2}$ diam. $4_{4}^{3}$, anfr. 11.;=R. supracostata, Garrelt, P. Ac. Phil., 1872, pl. 2, fig. 1, Viti, long. 10 mil.; ?=R. striolata, A. Adams, var.

4 N. Caledonia ; ex c. P. Bouvier, Esq.

5 Andamans $\left[15 \frac{3}{4}, 5\right]$; coll. J. Wood-Mason and G. Nevill, Esqrs.

Very close to R. striolata, A. Ad., Philippines, apparently only specifically separable by the constantly, markedly more developed sculpture of the upper whorls. The longitudinal ribs are in this form absent from the last four whorls, as in the type. Several of the Andaman specimens show the characteristic and peculiar dark "coating" so accurately described by Souverbie as " un enduit noir épais, analogue a celui qui récouvre certaines Mélanies;" this is especially the case with var. substriolata.

subvar. monstrosa, nov.

R. deformis, Sow. Junr. in Reeve, fig. 63, not R. deformis, Sow. Senr. in Generu of sihells, and Schwarlz. 
Very remarkable in having the appearance as if two portions of quite different specimens were artificially joined together! One specimen has the nine upper whorls typical, with the last unnaturally tumid and slightly out of the axis of the shell. The other, agreeing exactly with above quoted figure, has the eight upper and last whorls regular, with the antepenultimate strongly inclined to the left, altogether out of the proper axis. The longitudinal ribs are absent from the last four whorls.

2 Andamans $\left[16 \frac{1}{2}, 5 \frac{1}{2}\right.$; diam. anfr. penult. $\left.3 \frac{1}{\frac{2}{2}}\right]$; coll. J. Wood-Mason, Esq.

var. substriolata, nov.

The last three whorls are without longitudinal ribs.

4. Andamans $\left[13 \frac{1}{\frac{1}{4}}, 4 \frac{1}{2}\right]$; coll. Dr. F. Stoliczka, \&c.

1 Nicobars; coll. J. Wood-Mason, Esq.

var. convexior, nov.

More convex; aperture more everted ; spiral sculpture slightly more distinct; longitudinal ribbing on all but the last two whorls.

4 Andamans $\left[15_{4}^{\frac{3}{4}}, 5 \frac{1}{2}\right]$; coll. J. Wood-Mason and G. Nevill, Esqrs.

\section{Rissoina striolata.}

Rissoina striolata, A. Adams, P. Zool. S. 1S5̃2, Philippines; fide Schwarlz, long. 9, diam. 3 mil., anfir. 11.

5 Ceylon $\left[9 \frac{1}{2}, 3 \frac{1}{3}\right]$, Mauritius, and Andamans; coll. G. Nevill, Esq.

var. artensis.

Rissoina artensis, Montronzier, J. de. C., XX, N. Caledonia-long. S $\frac{1}{2}$, diam. $2 \frac{1}{2}$ mil., anfr. 10.

3 New Caledonia; ex c. P. Bouvier, Esq.

Scarcely separable even as a variety.

\section{Rissoina spirata.}

liissoa spirata, Sowerby, Gen. of Shells, 1824, pl. 20S, fig. 2; fide Schw. limg. 10:3, diam. 3-3 mil., from I. Rawak, Red Sea, and Plilippines; Suez, fide $M I^{\prime}$ Andrew; var.? = R. lamberti, Souverbie, $N$, Caledonia. 
3 Roweiah and Aden $[10,4]$; coll. J. B. Baxter and G. Ncvill, Esqrs.

var.

1 Aden $\left[8 \frac{1}{2}, 3 \frac{1}{2}\right]$; coll. G. Nevill, Esq.

\section{Rissoina orbignyi.}

Rissoina orbignyi, A. Adams, P. Zool. S. 1851, Luzon; fide Schw. long. 16.\%, diam. 5 mil., as R. d'Orbignyi.

var. subspirata, nov.

Long. $13 \frac{1}{2}$, diam. $5 \frac{1}{10}$ mil. Turriculate, like $R$. spirat a, but aperture everted and canaliculate at columellar base, as in true T. orbignyi.

4. Aden; coll. G. Nevill, Esq.

var. pachylabris, nov.

Anfr. 8 to 9 ; long. $11 \frac{1}{4}$, diam. $4 \frac{1}{10}$ mil., last whorl obsoletely spirally striate, last but one coarsely so, the others cancellated as in the normal form; a very broad callosity behind the thickened peristome ; aperture everted.

2 Annesley Bay [type var.]; coll. W. T. Blanford, Esq.

var. submarginata, nov. (? dist. sp.)

Acuminate, pointed, subturriculate; nine whorls slightly convex, the embryonal two smooth, rounded, mammillate, the next four closely and evenly reticulate, almost granulose, the last three somewhat distantly spirally striate, with a narrow groove just below the suture, the last one relatively very short, with spiral striation less distinct; aperture everted, columella short, abruptly bent back, behind the peristome a broad callosity.

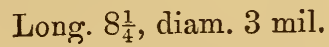

1 Andamans [type var.]; coll. G. Nevill, Esq.

\section{Rissoina percrassa.}

Rissoina percrassa, Nevill, Jour, Asiat. Soc. Bengal, 1874, pl. 1, fig. 13, Mauritius, long. 8, diam. 3 mil. 
1 Mauritius [type]; coll. G. Nevill, Esq.

One of the most remarkable species of the Genus.

Section G.-Zebina, II. and A. Adams.

Gen. Moll. I, p. 328, 1855, type R. coronata, Recluz; =Group VI, Schwartz, l. c., page 39.

\section{Rissoina tridentata.}

Rissoa tridentata, Michaud, Descr. Coq. nouv. 1832; fide Schwartz, long. 8, diam. 4 mil., from Red Sea, Philippines, Mauritius and Sandwich I.; Suez, fide $M Y^{\prime}$ Andrew; fide Pease $=R$. crassilabrum, Garrett, 1857; from Tahiti, fide Graffe.

2 N. Caledonia; ex. c. P. Bouvier, Esq.

1 Roweiah, Red Sea; coll. J. B. Baxter, Esq.

12 Mauritius $\left[9 \frac{1}{10}, 5\right]$, Andamans and Ceylon ; coll. G. Nevill, Esq.

4. Sandwich Islauds; coll. H. l’ease, Esq. $\left[6 \frac{1}{4}, 3 \frac{1}{4}\right]$.

\section{Rissoina coronata.}

Rissoina coronata, Recluz, ms., Schwartz, Denks. K. Ak. Wissen. Wien, $X I X, 1860$, Mauritius, long. 5.3, diam. 2.3 mil.; ?=R. bidentata, Phil., and var. from Upolu, fide Schmeltz, l. c. $V$.

1 Mauritius; coll. G. Nevill, Esq.

R. affinis, Garrett, P. Ac. Phil. 1873, Viti Is., long. 5 mil., must be close to this species?

\section{Rissoina bidentata.}

Rissoa bidentata, Phillipi, Wiegm. Arch. 1845, p. 64, Friendly Isl.,

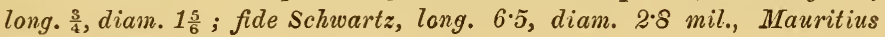
and Friendly Islands.

3 Mauritius $\left[5 \frac{3}{4}, 2 \frac{1}{2}\right]$; coll. G. Nevill, Esq.

Besides the dentition of the aperture, the differences in the shape of the spire, whorls, \&c., easily and certainly distinguish it from $R$. tridentata. 


\section{Rissoina semiplicata.}

Rissoina semiplicata, Pease, P. Zool. S. 1862, Howland Is., long. $3 \frac{1}{2}$, diam. $1 \frac{1}{2}$ mil., and Am.J. Conch. III, pl. XXIV,fig.29; Conch.-Cab. II, pl. 15A, fig. 2 .

1 Upolu; ex Mus. Godeffroy $\left[4,1 \frac{3}{4}\right]$.

3 Paumotus; coll. H. Pease, Esq.

Possibly better classed as a var. of the preceding, the two denticulations within the aperture are quite distinct, though not recorded in Pease's descriptions. It can always be distinguished by the markedly shorter spire, more tumid last whorl, less everted aperture and especially by the ribs on the penultimate whorl; the above will also distinguish it from $R$. coronata, Recl., possibly a mere var. of $R$. bidentata.

\section{Rissoina moravica.}

Rissoina moravica, Hörnes, Foss. Moll. Tert. Wien, 1856.

1 Steiuabrunn; coll. Herr Schwartz von Mohrenstern.

\section{Rissoina peasiana.}

Rissoina peasiana, G. Nevill, Weinkauff, Conch.-Cab. II, 1881, pl. 15A fig. 6, Rarotonga.

A fine species of ten whorls, quite distinct from $R$. tridentata [=curta $]$, the whorls are scarcely convex, the last five whorls smooth, the three preceding coarsely ribbed; no teeth within the aperture, a small dentiform thickening on the parietal margin, near the outer lip; some resemblance to $R$. macrostoma, Desh. [fossil].

Long. 12, diam. 5 mil. [the two apical whorls wanting].

1 Rarotonga [type]; ex Mus. Godeffroy [as “R. ? curta, Sow.”]

\section{Rissoina subulina.}

Rissoina subulina, Weinkauff, Conch. Cab. II, 1881, pl. 15A, fig. 3, Japan -long. $3 \frac{3}{4}$, diam. $1_{2}^{\prime}$ mil.

2 Japan [type]; ex Mr. R. Damon ["coll. A. Adams"]. 


\section{Rissoina eulimoides.}

Rissoina eulimoides, A. Adams, P. Zool, S. 1851, Philippines; fide Schvo. long. 6, diam. 2.6 mil.

1 Andamans; coll. Capt. R. C. Temple.

1 Upolu; ex Mus. Godeffroy.

5 Ceylon $\left[6 \frac{1}{2}, 3\right]$; coll. G. Nevill, Esq.

I can perceive no trace of sculpture on the two upper whorls in auy of these, even with the lens, as is recorded by Herr Schwartz, neither is there any mention of such sculpture in the original description.

1 New Caledonia; ex c. J. Caldwell, Esq.

83. Rissoina stoppanii.

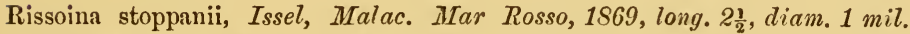
subfossil $;=\mathbb{R}$. oryza, Garrett, P. Ac. Philad. 1873, Viti Isl., long. 2 snil.

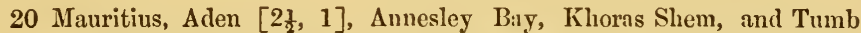
Island [Persian Gulf]; coll. IV. T. Blanford and G. Nevill, Esqrs.

\section{Rissoina sublævigata.}

Rissoina sublævigata, G. Nevill, J. A. S. B. 18S1, Andamans, long. 31, diam. $1 \frac{1}{2}$ mil., and Weinkauff, Conch.-Cab. II, 1SS1, pl. 15C, fig. \%.

6 Andamans [type]; coll. G. Nevill, Esq.

\section{Section H.-Pyramidelloides, nov.}

Type R. Miranda, A. Adams.

\section{Rissoina insolita.}

Rissoina insolita, Deshayes, Cat. Moll. Reunion, 1863-long. 3, diam.1 mil.

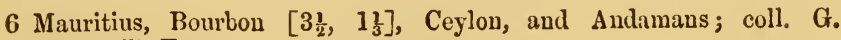
Nevill, Esq. 
15 Arakan and Bombay $\left[3,1 \frac{1}{1}\right]$; coll. Rev. S. B. Fairbank and W. T. Blanford, kisq.

The spire is often a trifle inclined to the right, and out of the axis.

6 Persian Gulf; ex c. Colonel G. B. Mainwaring.

subvar. deformis, nov.

Spire notably bent away from the axis, somewhat forward and to the left, as in many of the Eulimidae.

1 Mauritius; coll. G. Nevill, Esq.

var major, nov.

3 Mauritius $\left[4 \frac{1}{4}, 1 \frac{3}{4}\right]$; coll. G. Nevill, Esq.

var. depauperata, nov.

4 Mauritius [21 $\frac{1}{2}$, vix. 1); coll. G. Nevill, Esq.

1 Singapore; coll. Dr. F. Stoliczka.

86. Rissoina miranda.

Rissoina miranda, A. Adams, Ann. Mag. VIII, 1861, Mino.Sima, 63F.; $?=R$. insolita, Desh.

I can detect no marked difference in this form from the preceding, except that the nodules are less rounded or pearllike.

2 Hong-Kong; coll. R. Hungerford, Esq.

2 Japau [typical]; ex Mr. Damon [coll. A. Adams.]

Long 3, diam, 1 mil.

\section{Rissoina bellardii.}

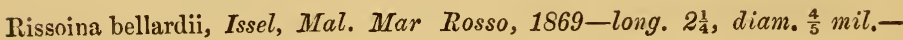
subfossil; ? R. insolita, Desh., var.

Perhaps only a variety of the preceding, distinguished by shorter and more convex spire, more tumid last whorl, \&c.

1 Jashk, Persian Gulf $\left[1 \frac{3}{4}, \frac{3}{5}\right]$; coll. W. T. Blanford, Esq.

4 Persian Gulf ; ex c. Colonel G. B. Mainwaring. var.?

1 Annesley Bay, Red Sea $\left[2, \frac{1}{2}\right]$; coll. W. T. Blanford, Fsq. 
Sub-Genus IRAVADIA, Blanford.

J. Asiat. Soc., Bengal, 1867, p. 56, type I. ornata, Blf.; fide Stoliczka, belongs to sub-fam. Rissoince [anim. ign.].

\section{Rissoina [Iravadia] ornata.}

Iravadia ornata, W. T. Blanford, J. Asiat. Soc., Bengal, 186\%, Irawady Delta.

30 Pt. Canning and Irawady Delta [typical]; coll. W. T. Blunford aud J, Wood-Mason, Esqrs.

\section{Rissoina [Iravadia] trochlearis.}

Rissoina trochlearis, Gould, P. Bost. S. 1861, China Seas-axis 16, diam. -0\%3 poll.; =R. annulata, Dunker, Japan.

1 Japan; ex c. Mr. Damon [coll. A. Adams; labelled "Onoba cingulifera, A. Ad."]

12 Hong-Kong, under stones at extreme low water; coll. R. Hungerford, Esq. $[4,2]$.

40 Ceylon, Aden, Bombay, Andamans, Singapore, and Arracan; coll. F. Stoliczka and G. Nevill, Esqr's.

var. minor. [? dist. sp.]

Conch.-Cab. II, pl. 15 B, fig. 2.

Shell like that of a miniature $R$. ornata, covered with a thin epidermis. Thick, solid, without the minute, decussating striø of the preceding; whorls six, the first two without sculpture, the next two with two very strong spiral keels, the fifth with three, the last of which is less developed than the other two, the last with six of these sulcations; outer lip much thickened, fimbriated [as it were] by five of these projecting sulcæ; margins of aperture without any basal notch or canal, joined by a callosity; columella nearly straight.

Long. $3 \frac{1}{4}$, diam. $1 \frac{1}{2}$ mil. [Kesm]. Long. $3 \frac{1}{8}$, diam. $1 \frac{1}{2}$ mil. [Hong-Kong]. 
60 Gwadar, Tavila and Kesm [Persian Gulf]; coll. W. T. Blanford, Esq.

4. Hong-Kong; coll. R. Hungerford, Esq.

20 Bombay; coll. Rev. S. B. Fairbank.

1 Kathiawar; coll. F. Fedden, Ess.

\section{Rissoina [Iravadia] n. sp.}

2 Bombay; coll. Rev. S. B. Fairbank. [anfr. 7 ; long. 1 $\left.\frac{3}{4} \mathrm{~m}.\right]$

\section{Sub-Genus ISSELIELLA, Nevill.}

Isseiia, O. Semper ms., Schmeltz, Cat. Mus. Godeffroy, $V$ [sine desc.], type R. mirabilis Dkr. [not Isselia, Bourg. Descr. nouv. Genres, \&c. 1877].

Distinguished by the very distinct basal "canal " [or notch], twisted columella and sinistrally bent, pyramidella-like apex. Type Rissoina? abnormis, Nevill.

Perhaps Alvania bourguignati, Issel, belongs to this group ?

A. Adams (ms.) and von Martens, in Möbius Reise Mauritius, \&c., consider these forms as allied to-or part of-Lachesis. O. Semper, fide Schmeltz, placed them in the Family Planaxidae. I feel convinced, and I believe Dr. Weinkauff is of the same opinion, that they belong to the Rissoidce and are closely allied to true Rissoina.

\section{Rissoina [Isseliella] mirabilis.}

Rissoina mirabilis, Dunker, Cat. Mus. Godef. III, Samoa Islands.

3 Upolu; ex Mus. Godeffiroy.

\section{Rissoina [Isseliella] pseudoconcinna, n. sp.}

Rissoina concinna, Sow. [as of A.Adams, ms.] in Con. Icon. 1879, pl. 1, fig. 9, Japan [not $R$. concinna, A. Ad. 1851].

1 Japan, ex c. Mr. Damon [coll. A. Adams; labelled Lachesis n. sp.!]

\section{Rissoina [Isseliella] abnormis.}

Rissoina? abnormis, G. Nevill, Jour. Asiat. Soc., Bengal, 1875, p. 100, pl. VIII, fig. 23, Mauritius-long. 3, diam. $1_{3}^{\frac{1}{3}}$ mil.; Lachesis sp. fide Martens. 
14 Mauritius [type], Ceylon, and Seychelles; coil. G. Nevill, Esq.

6 Andamans and Bombay; coll. W. T. Blanford and J. Wood. Masou, Esqrs.

\section{Genus FAIRBANKIA, W. T. Blanford.}

Ann. Mag. 1568 [Dec.], type F. bombayana, Blf.

Belongs to the Family Rissoellida, fide Martens, Zoological Record V.; Stoliczka placed it in the sub-family Rissoinœe.

\section{Fairbankia bombayana}

Fairbankia bombayana, W. T. Blanford, Ann. Mag., 186S-long. 7, diam. 3 [vix], Bombay and small var. 5, 2.

20 Bombay Harbour; coll. Rev. S. B. Fairbank and W. T. Blauford, Esq.

var. depauperata, nov.

2 Bombay $\left[4 \frac{3}{4}, 2\right]$; coll. Rev. S. B. Fairbank.

\section{Fairbankia [?], n. sp.}

Spirally sculptured, thin epidermis-long. $4 \frac{1}{2}$, diam. $1_{\overline{1}}^{9}$ mil.

1 Arakiu [type]; coll. Dr. F. Stoliczka.

\section{Fairbankia [?] feddeniana.}

Fairbankia ? feddeniana, G. Nevill, Jour. As. Soc., Bengal, 1881, Kathiawar.

1 Kathiawar [type]; coll. F. Fedden, Esq.

1 Kura Salt Works, Kathiawar [10 feet bulow the surface]; coll. F. Fedden, Esq.

$$
\text { ? var. [? spec. juv.]. }
$$

4 Kura Salt Works; coll. F. Fedden, Esq. 


\section{Genus FOLINIA, Crosse.}

J. de C., 1867, type Rissoa insignis, Folin, from Panama.

Two species have since been described from Suez in the "Fonds de la Mer," but not figured! I have never seen the Genus.

\section{Genus RISSOA, Fréminville, ms.}

Desmarest, Bull. Sci. Soc. Philom. 1814, type R. violacea, Desm.; type fide Gray, P.Z.S. 1847, R. labiosa [= membranacea]; type fide Herrm. 1848, Turbo cimex; sect.= Mohrensternia, Stol., Palaont. Ind. 186\%, type $R$. angulata, Eichw.; sect. =Zippora, Leach, Syn. Moll. Gt. Brit. 1852, type R. auriscalpium, Lin. ; sect.=Rissostomia, Sars, Moll. Norveg. 1878, type R. membranacea, J. Ad. [as Z. drummondi, Leach.]

\section{Rissoa sulzeriana.}

Alvania sulzeriana, Risso, Hist. Nat. Mer. 1826.

3 Piedmont [fossil]; coll. Herr Schwartz von Mohrenstern.

\section{Rissoa lachesis.}

Turbo lachesis, Basterot, Mém. Geol. Bord., 1825.

5 Steinabrunn [fossil]; coll. Herr Schwartz von Mohrenstern.

\section{Rissoa albella.}

Rissoa albella, Lovén, Index Moll. Scand., 1846, Bohnsläu; var.=R. sarsii Lov., Bergen, fide Jeffreys.

6 "Kattegat;" coll. Herr Schwartz von Mohrenstern.

\section{Rissoa decolorata.}

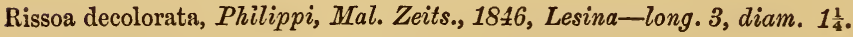

3 Mediterranean; coll. Herr Schwartz von Mohrenstern.

\section{Rissoa interrupta.}

Turbo interrupta, J. Adams, Trans. Lin. Soc. V, 1798; fide Jeffreys $=R$. parva, var. 
30 England, \&c; ex c. As. Soc. Bengal, Dr. F. Stoliczka and G. Nevill, Esq.

\section{Rissoa lilacina.}

Rissoa lilacina, Récluz, Rev. Zool. Cuv. 1843, for Turbo violaceus, Mühlfeld, 1824 [not R. violaceus, Desm. 1814] ; fide Jef. $=R$. violacea, Desm.

18 N. W. France; coll. Miss Beaufort.

\section{Rissoa lineolata.}

Rissoa lineolata, Michaud, Descr. Coq., 1832, Provence.

4. Mediterranean; ex c. Herr Schwartz von Mohrenstern.

\section{Rissoa membranacea.}

Turbo membranaceus, J. Adams, Linn. Trans. V, 1797; fide Jeffreys, vars. minor, venusta, Phil. and elata, Phil.; sect. Rissostomia.

2 Mediterranean; ex c. Herr Schwartz von Mohrenstern.

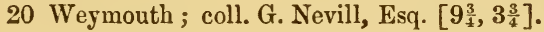

var. labiosa.

Helix labiosa, Montagu, Test. Brit., 1803.

18 N. W. France and Weymouth; coll. Miss Beaufort and G. Nevill, Esq.

\section{Rissoa grossa.}

Rissoa grossa, Michaud, Desc. Coq., 1832, Provence; = R. membranacea, fide Jeffreys; sect. Rissostomia.

9 Algiers; coll. P. Joly, Esq.

\section{Rissoa violacea.}

Rissoa violacea, Desmarest, Bull. Soc. Philom. 1814; vars. ecostata and porifera, fide Jeffreys, also $=R$. guerini and lilacina, Recl. \& c.

12 Algiers; coll. P. Joly, Esq.

6 Mediterranean; ex c. Herr Schwartz von Mohrensteru. 


\section{Rissoa oblonga.}

Rissoa oblonga, Desmarest, Bull. Soc. Philom., 1S14; =R. membranacea, fide Jeffieys; sect. Rissostomia.

8 Algiers; coll. P. Joly, Esq.

4 Mediterranean; ex c. Dr. F. Stoliczka and Herr Schwartz von Molirenstern.

3 Ajaccio; coll. G. Nevill, Esq.

\section{Rissoa monodonta.}

Rissoa monodonta, Bivona, ms., Philippi, Enum. Moll. Sicil., 1836.

15 Algiers; coll. P. Joly and G. Nevill, Esqrs.

30 Mediterranean; ex c. Dr. F. Stoliczka and Herr Schwartz von Molirenstern.

\section{Rissoa ventricosa.}

Rissoa ventricosa, Desmarest, Bull. Soc. Philom., 1S14; = R. memlranacea, fide Jeffreys.

50 Algiers, Ajaccio, \&c.; coll. G. Nevill, Esq., P. Joly, Esq., and Herr Schwartz von Mohrenstern.

\section{Rissoa radiata.}

Rissoa radiata, Philippi, Enum. Moll. Sicil. I. 1836.

30 Algiers; coll. P. Joly, Esq.

var. lævigata.

6 Algiers; coll. P. Joly, Esq.

\section{Rissoa rufilabrum.}

Rissoa rufilabrum, Leach, ms., Alder, Ann. Mag. XIII, 1844, England ; fide Jeffreys $=R$. violacea, Desm.

4 Weymouth; coll. G. Nevill, Esq.

\section{Rissoa variabilis.}

Turbo variabilis, Mïhlfeld, Berlin Verh. IV, 1824; Rissoa costata, Desm., Bull. Philom. 1814, not Turbo costatus, Lin. [Alvania sp.]

30 Mediterranean; ex c. Dr. F. Stoliczka and Herr Schwartz von Mohrenstern. 
I have followed Herr Schwartz in not considering it desirable to change the specific name to costata.

\section{Rissoa subcostulata.}

Rissoa subcostulata, Schwartz von Mohrenstern, Denks K. Ak. Wissens. Wien, XIX, 1860, for $R$. costulata, Alder, 1844, not of Risso $1826[=R$. variabilis, Mhlf.]

29 Weymouth, \&c.; coll. G. Nevill, Fsq.

var. sublævis.

28 Algiers, \&c., Mediterranean; coll. P. Joly, Esq., and Herr Sclıwartz von Mohrenstern.

Some of these were sent me by M. Joly as "var. lavis."

\section{Rissoa pulchella.}

Rissoa pulchella, Philippi, Enum. Moll. Sicil., 1836.

6 Mediterranean : coll, Herr Schwartz von Mohrenstern.

19. Rissoa similis.

Rissoa similis, Scacchi, Catal. p. 14, 1S36, Italy; fide Jeffreys, small var. of R. costulata, Alder.

30 Mediterranean; ex c. Dr. F. Stoliczka and Herr Schwartz von Mohrenstern.

15 Algiers; coll. P. Joly, Esq.

var. lævis.

R. similis var. lavis, Walson, P. Zoul. S. 1873, Madeira; fide Monter. R. geminula, Fischer, Fonds de la Mer, pl. XXIII, Gulf Gascony.

16 Algiers; coll. P. Joly, Esq.

20. Rissoa parva.

Turbo parvus, $D a$ Costa, Brit. Conch. 1779 ; fide Jeffreys, vars. interrupta Ads. and exilis, also = Cingula alba, Fleming, \&c.

100 Mediterranean, N. W. France, England, and Biarritz; coll. Miss Beau. fort, Herr Schwartz von Molirenstern, and G. Nevill, Esq. 


\section{Rissoa algeriana.}

Rissoa Algeriana, Monterosato, J. de C., 187\%, Algiers, pl. III, fig. 5, "coll. Joly."

20 Algiers [typical]; coll. P. Joly, Esq.

var. rufula.

R. Algeriana, var. rufula, Monterosato, $l, c$.

9 Algiers; coll. Mons. Joly.

22. Rissoa pusilla.

Rissoa pusilla, Philippi, Enum. Moll. Sicil. 1836 ; fide Schwartz, R. dolium, Nyst, 1843.

10 Algiers; coll. P. Joly, Esq.

23. Rissoa desmaresti.

Rissoa desmaresti, Audouin, Descr. de l'Egy p'e, pl. III, fig. 21, 1826. mil.

The outline figure of type, natural size, measures about 3

var. minima.

1 Annesley Bay; coll. W. T. Blanford, Esq.

Long. 1 mil.

\section{Rissoa auriscalpium.}

Turbo auriscalpium, Linnaus, Syst. Nat. Ed. XII, 1757 ; sect. Zippora.

30 Algiers, \&c., Mediterranean; coll. Herr Schwartz von Mohrenstern, and P. Joly, F. Stoliczka, and G. Nevill, Esqrs.

25. Rissoa (?) rudis.

Rissoa rudis, Phillippi, Enum. Moll. Sicil. I, 1836.

50 Algiers; coll. P. Joly, Esq.

Not included in Rissoa [restricted] by Schwartz, but considered an Alvania? 
var. minor.

6 Algiers; coll. P. Joly, Esq.

\section{Rissoa inconspicua.}

Rissoa inconspicua, Alder, Ann. Mag. XIII, 1844, Northumberland.

4. Algiers ; coll. P. Joly, Esq.

\section{Rissoa angulata.}

Rissoa angulata, Eichwald, Nat. Skiz. Lithauen. 1S30; sect. Mohren. sternia.

1 Gayà [fossil]; ex c. Herr Schwartz von Mohrenstern.

\section{Rissoa splendida.}

Rissoa splendida, Eichwald, Nat. Hist. I, 1S30, p. 219, Black Sea.

4 Theodosia, Black Sea; ex c. C. F. Ancey, Esq.

\section{Rissoa (?) globulus.}

Rissoa globulus, Möller, Ind. Moll. Groenl. 1842.

1 Greenland; ex "Linmæa."

Looks to me more like a form of brackish-water Hydrobiince.

Genus ALVANIA, Risso.

Hist. Eur. Mérid. IV, 1826, p. 140, type A. cimex, Lin. [as Alv. europæea, Risso], type restr. Gray, P. Z. S. 1847 [as A. calathiscus]; sect.= Man. zonia, Brusina, Chieregh. Conch. 1870, type Turbo zetlandicus, Mtg. [= Turboella, Leach, ms., Gray, P. Z. S. 1847]; sect. = Plagiostyla, P. Fischer, Fonds de la Mer, et Act. Soc. Lin. Bord. XXIX, 1869, type Rissoa lactea, Mich.

\section{Alvania adelæ.}

Rissoa adelæ, d'Orbigny.

2 Steinabrunn [fossil]; coll. Herr Schwartz von Mohrenstern. 


\section{Alvania moulinsi.}

Rissoa moulinsi, d'Oröigny.

4 Steinabrunn [fossil]; coll. Herr Schwartz von Mohrenstern.

\section{Alvania zetlandica.}

Turbo zetlandicus, Montagu, Trans. Lin. Soc., XI, 1803, Gt. Britain; sect. Manzonia.

5 Steiuabrunn [fossil]; coll. Herr Schwartz von Mohrenstern.

\section{Alvania brevis.}

Rissoa brevis, Allioni.

5 Sienna [fossil]; coll. Herr Schwartz von Mohrenstern.

\section{Alvania mariæ.}

Rissoa mariæ, d'Orbigny.

2 Steinabrunn [fossil]; coll. Herr Schwartz von Mohrenstern.

M. Joly considers this species to be found living at Algiors and not separable specifically from $A$. reticulata.

\section{Alvania scalaris.}

Rissoa scalaris, Dubois.

3 Steinabrunn [fossil]; coll. Herr Schwartz von Mohrenstern.

\section{Alvania venus.}

Rissoa venus, d'Crbigny.

4. Steinabrunn [fossil]; coll. Herr Schwartz von Mohrenstern.

\section{Alvania cimex.}

Turbo cimex, Linneus, Syst. Nat. X, 1758; fide Jeffreys $=$ Turbo calathiscus, Mtg. = Alv. europaa, mammillata, and freminvillea, Risso, $=R$. cancellata, Desm. $=$ R. granulata, Phil.

30 Algiers and Ajaccio; coll. G. Nevill, Esq.

30 Loc. ?; ex c. Dr. F. Stoliczka and G. Nevill, Esq. 
var. depauperata.

Rissoa cimex, var. depauperata, Monterosato, J. de C., 15\%\%, Algiers.

8 Algiers; coll. P. Joly, Esq.

\section{Alvania cancellata.}

Turbo cancellatus, DaCosta, Brit. Conch. 17\%8; = R. crenulata, Mich. 1832.

6 Ajaccio and Algiers; "coll. G. Nevill and P. Joly, Esqr's.

14 England; coll. G. Nevill, Esq.

\section{Alvania cimicoides.}

Risson cimicoides, Forbes, Report Brit. Ass. for 1S43, Fgean Sen; fide Jeff. var. minima, Plymouth.

2 England; ex c. G. Nevill, Esq.

\section{Alvania costata.}

Turbo costatus, J. Adams, Trans. Lin. Soc., III, 1S00, England [not R. costata, Desm.] ; fide Weinkauff $=R$. exigua, Mich. $=R$. carinata, Phil.; sect. Manzonia.

16 Mediterranean ; ex c. G. Nevill, Esq. $\left[3,1 \frac{1}{8}\right]$.

20 Algiers; coll. P. Joly, Esq.

$$
\text { ? var. [? dist. sp.] }
$$

6 Loc.?; ex. c. Dr. F. Stoliczka $\left[2, \frac{3}{4}\right]$.

\section{Alvania lactea.}

Rissoa lactea, Michaud, Descr. esp. Riss. 1832 ; = Turbo cancellatus, Lam. [not DaC.]; sect. Plagiostyla, fide Fischer.

12 Mediterranean; coll. Herr Schwartz von Mohrenstern.

3 N. France; coll. Miss Beaufort.

\section{Alvania montagui.}

Rissoa montagui, Payrandeau, Cat. Moll. Corse, 1826.

50 Algiers, \&c., Mediterranean : coll. P. Joly and G. Nevill, Esqrs., aud Herr Schwartz von Mohrenstern.

20 Lnc. ?; ex c. Dr. F. Stoliczka. 
var. lineata.

Alvania lineata, Risso, Hist. Moll. Europ. Mérid, 1826.

16 Algiers; coll. P. Joly, Esq. [as " R. lineata, Risso"].

\section{Alvania scabra.}

Rissoa scabra, Phillippi, Enum. Moll. Sicil. 1836.

2 Palermo; coll. P. Joly, Esq.

15. Alvania reticulata.

Turbo reticulatus, Montagu, Test. Brit. 1803 [not of Cpr. 1864]; fide Jef. $=R$. beani, Hanley.

8 Algiers and Marseilles; coll. P. Joly, Esq. [as " $R$. reticulata-var. calathus"'].

2 England; ex c. G. Nevill, Esq. [labelled " $R$. beani, Hanl "].

3 Algiers; coll. P. Joly, Esq. [as " $A$. reticulata, var. maria, Orb.”]

16. Alvania striatula.

Turbo striatulus, Montagu, Test. Brit., 1803 [not T. striatulus, Lin.].

3 N. France and England; coll. Miss Beaufort, and G. Nevill, Esqr.

17. Alvania sp.?

4 Mediterranean; coll. Herr Schwartz von Mohrenstern.

Can this be Alv. schwartziana of Brusina? It was sent to the museum as "A. subcrenulata, Schw." [ubi ?].

\section{Alvania [??] mariella.}

Alvania mariella, A. Adams, Ann. Mag. VIII, 1861, Tsu.sima, 26 fths. and Korea Strait, 46 fths.

1 Japan [typical]; ex Mr. Damon [coll. A. Adams].

Long. 2, diam. 1 mil. A beautiful form, agreeing exactly with the original description; the correct generic classification is very doubtful.

\section{Alvania cælata.}

Alvania cælata, A. Adams, Ann. MIag., TIII, 1861, Tsu-sima, 25 fths. 
1 Japan [typical]; ex Mr. R. Damon [coll. A. Adams].

Seems to be an immature specimen; long. $2 \frac{1}{4}$, diam. $1 \frac{1}{3} \mathrm{mil}$.

20. Alvania badia [?].

Alvania badia, A. Adams, Ann. Mag. VIII, 1S61, Kalahai and Shan-tung.

1 Japan; ex Mr. Damon [coll. A. Adams].

\section{Alvania ferruginea.}

Alvania ferruginea, A. Adams, Ann. Mag. VIII, 1861, Yesso, 7 fths.

2 Japan [typical]; ex Mr. Damon [coll. A. Adams].

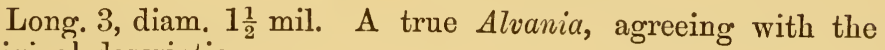
original description.

\section{Alvania fenestrata.}

Rissoa fenestrata, Krauss, Sud-Afrik. Moll. 1S4S, "on the byssus of Pinna squamifera," Emb. River Kysna-1.5, 0.8 lin. [not of Gould].

2 Port Natal; coll. G. Nevill, Esq.

23. Alvania n. sp.

10 Mauritius; coll. G. Nevill, Esq.

Long. 2, diam. 1 mil. Near the preceding.

\section{Alvania n. sp.}

3 Mauritius; coll. G. Nevill, Esq.

Closely allied to the next species.

\section{Alvania mauritiana.}

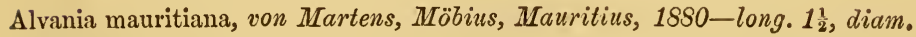
$\frac{3}{4}$ mil.

2 Mauritius; coll. G. Nevill, Esq. 
26. Alvania n. sp.

Ciet. Gast. S. India, I. 1867, p. 274, as "sp. which extremely resembles the fossil species of Mohrensternia, but possibly can be a form of Alaba."

20 Arakan; coll. Dr. F. Stoliczka.

6 Bombay; coll. Rev. S. B. Fairbank.

27. Alvania n. sp.

2 Balapiti, Ceylon; coll. G. Nevill, Esq.

28. Alvania sp.

1 Ammesley Bay; coll. W. T. Blanford, Esq.

Possibly A. desmaresti, Audouin-var.?

29. Alvania n. sp.

1 Mauritius; coll. G. Nevill, Esq.

30. Alvania n. sp.

10 Galle; coll. J. B. Baxter, Esq.

Very prettily maculated with brown.

31. Alvania n. sp.

2 Gwadar; coll. W. T. Blanford, Esq.

\section{Alvania carinata.}

? Turbo carinatus, DaCosta, Brit. Conch. 17\%8; fide Monterosato, J. de C. $1877=$ R. labiata, Phil.

2 Algiers; coll. P. Joly, Esq. [as “Rissox carinata, DaCosta-very rare"].

Can this be the Turbo striatulus, Mtg. = Turbo carinatus, DaCosta = Turbo monilis, Fleming? I doubt it.

\section{Alvania mutabilis.}

Alvania mutabilis, Schwartz von Mohrenstern, ms., Weinkauff, Conch. Medit. 1S68, Algeria and Dalmatia. 
12 Algiers; coll. P. Joly, Esq.

Fide Monterosato, distinct from A. canariensis, Orb.

\section{Alvania caribæa.}

Rissoa caribæa, d'Orbigny .

8 Algiers; coll. P. Joly, Esq.

\section{Alvania sculptilis.}

Rissoa sculptilis, Monterosato, J. de C., 18\%8, Algiers, "coll. Joly."

1 Algiers [typical]; coll. P. Joly, Esq. [“very rare"].

\section{Alvania [?] circinata [?]}

Alvania circinata, A. Adams, Ann. IIag., VIII, 1861, Isl. Sado, 30 fths.

1 Japan; ex Mr. Damon [coll. A. Adams-labelled "A. circinata"].

Long. $1 \frac{1}{2}$, apparently not adult, the lip is not coloured, and, altogether, it does not agree with the original description.

\section{Alvania scitula.}

Alvania scitula, A. Adams, Ann. Mag., 1861, Lo-shan-kow and Shan-tang.

2 Japan [typical]; ex Mr. Damon [coll. A. Adams].

Long. $2 \frac{1}{4}$, diam. $1 \frac{1}{3}$ mil. Agrees well with the description. I shall give, when I have the opportunity, the measurements of A. Adams' species, which he omitted in his original description.

\section{Alvania interfossa.}

4. Arakan; coll. W. T. Blanford, Esq.

2 Ceylon; coll. G. Nevill, Esq.

39. Alvania n. sp.

A pretty little form, here and there maculated with brown.

2 Galle; coll. J. B. Baxter, Esq. 
40. Alvania n. sp.

20 Singapore; coll. Dr. F. Stoliczka.

Long. $1 \frac{1}{2}$, diam. 1 mil. Unusually broad, in proportion.

var robusta.

30 Bombay; ex c. Dr. F. Stoliczka.

41. Alvania n. sp.

The whorls are markedly less convex than in the Nicobarese $A$. stigmata, Frauenfeld.

8 Bombay $\left[1 \frac{3}{4}, 1\right]$; coll. Rev. S. B. Fairbank, and W. T. Blauford, Esq.

4 Andamans, Ceylon, and Mauritius $[2,1]$; coll. G. Nevill and J. B. Baxter, Esqr's.

4. Arakan; coll. Dr. F. Stoliczka.

42. Alvania n. sp.

4 Arakan and Penang; coll. Dr. F. Stoliczka.

1 Singapore $\left[2,1 \frac{1}{8}\right]$; coll. Dr. F. Stoliczka.

Longitudinal ribs quite straight, rather distant and thick.

43. Alvania stigmata.

Alvania stigmata, von Frauenfeld, Moll. Novara Reise, $1 S 66$ [Kamorta, Nicobars]-long. 2, diam. mil., on expl. of plate as A. stigmatica!

var. tumida [? dist. sp.]

6 Andamans; coll. J. Wood-Mason and G. Nevill, Esqrs.

2 Ceylon; coll. G. Nevill and J. B. Baxter, Esqrs.

20 Arakan; coll. Dr. F. Stoliczka.

\section{Alvania weinkauffii.}

Alvania weinkauffi, Schwartz, ms., Weinkauff, Conch. Medit., 186S, Algeria. 20 Algiers; coll. P. Joly, Esq.

Fide Monterosato, distinct from Alv. dictyophora, Philippi? 


\section{Alvania lanciæ.}

Rissoa lanciæ, Calcara, Moll. Sicil. $1845 ;=R$. philippiana, Jeffreys, Ann. Mag. 1856, Spezzia.

30 Algiers; coll. P. Joly and G. Nevill, Esqrs.

1 Mediterranean; coll. Herr G. Schwartz von Mohrenstern [as "Alv. philippiana"].

var. tessellata.

Alvania tessellata, Schwartz, from Algiers.

1 Algiers; coll. P. Joly, Esq.

46. Alvania n. sp.

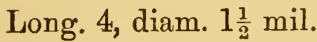

2 Hongkong; coll. R. Hungerford, Esq.

Gould's description of Alvania fusca, from Hongkong, would answer perfectly to this form, but is said to have eight whorls and measure long. 5, diam. $1 \frac{1}{2}$ mil. Dr. Hungerford's shell has only five and a half whorls.

\section{Aivania [?] asturiana.}

Plagiostyla asturiana, P. Fischer, Act. Soc. Lin. Bord., XXIX, 1869, [?] and Fonds de la Mer, pl. II, fig. 5, 1872, Gulf of Gascony; sect. Plagiostyla.

1 Gijon, Bay of Biscay; coll. Marquis de Folin.

If M. Fischer had made this remarkable species the type of his Genus Plagiostyla, instead of $R$. lactea, Michaud, I should have considered the form as worthy of at least subgeneric distinction.

\section{Genus FENELLA; $A$. Adams.}

Ann. Mag., 1860, type F. pupoides, A. Ad., as "Finella," emend. 1864.

The Rissoa semicostata, Pease, A. J. C., 1868, Caroline Irl., $3,1 \frac{1}{2}$, seems to belong here? A. Adams has also described $F$. candida, $1861 ; F$. asperulata, $1860 ; F$. pulchella, $1860 ; F$. ferruginea, 1860, all from Japan. 


\section{Fenella rufocincta.}

Dunkeria rufocincta, A. Adams, Ann. Mag. VIII, 1861, Mososeki, Tabane and Takano-Sima; Suez, fide M'Andrew.

2 Japan; ex Mr. R, Damon ["coll. A. Adams"].

Long. $3 \frac{3}{4}$, diam. $1 \frac{1}{6}$ mil.

\section{Fenella rissoiformis.}

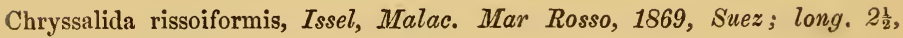
diam. 1 mil.

2 Massaua; coll. Prof. A. Issel.

2 Andamans; coll. G. Nevill, Esq.

1 Roweiah, Red Sea; coll. J. B. Baxter, Esq.

This last is a rather narrower form.

\section{Fenella cerithina.}

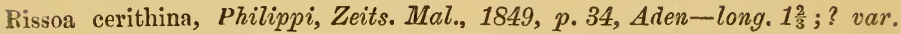
$=F$. scabra, A. Ad.

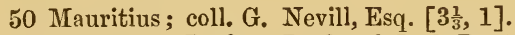

5 Suez; coll. Prof. A. Issel and J. B. Baxter, Esq.

8 Aden; coll. Dr. F. Stoliczka and G. Nevill, Esq.

2 Natal; coll. G. Nevill, Esq.

Herr Schwartz identified these Natal specimens as $F$. excurvata [ubi?]; Gould speaks of an Alvania excurvata, Cpr.

10 Oman and Tawila [Persian Gulf]; coll. W. T. Blanford, Esq.

var. [? dist. sp.].

26 Singapore; coll. Dr. F. Stoliczka.

\section{var. scabra.}

Dunkeria scabra, A. Adams, Ann. Mag. VI, 1860, p. 421, Tsu-sima, Anfr. $8 \frac{1}{2}$, ["liris elevatis tribus"]; Suez, fide McAndrew.

2 Japan; ex Mr. Damon [" coll. A. Adams"].

Long. $2 \frac{1}{2}$, diam. 1 mil., the other rather larger. 
40 Andamans, Bombay, Galle and Mauritius; coll. W. T. Blanford, J. B. Baxter, and G. Nevill, Esqrs.

I am not clear whether this is, or is not, a distinct species from the preceding $[F$. cerithina]? They seem to me to run one into the other! Certain it is, however, that Mr. A. Adams identified as $F$. scabra, indubitable specimens of $F$. cerithina, which had been dredged by Mr. McAndrew at Suez! The sculpture of the Japan forms appears somewhat coarser, and with one transverse stria less.

10 Hongkong; coll. R. Hungerford, Esq.

6 Kathiawar; coll. F. Fedden, Esq.

\section{Fenella sp.}

2 Hongkong; coll. R. Hungerford, Esq.

Something like $F$. reticulata, A. Adams.

\section{Fenella sp.}

3 Hongkong; coll. R. Hungerford, Esq.

\section{Fenella [?] n. sp.}

1 Andamans; coll. G. Nevill, Esq.

Probably one of the most minute marine Mollusca in existence.

\section{Fenella virgata.}

Rissoa virgata, Philippi, Zeits. Mal. 1849, Suez, Aden, \&c., and Descr. de l'Egypte, pl. III, fig. 29, long. 2.

This is almost certainly one of A. Adams' species, perhaps his $F$. pupoides?

\section{var. [?]}

2 Mauritius; coll. G. Nevill, Esq.

$$
\text { var. [?] }
$$

1 Mauritius; coll. G. Nevill, Esq. 


\section{Fenella sp.}

Pupoides? vinacea var.

50 Seychelles, Mauritius, and Natal; coll. G. Nevill, Esq.

12 Bombay; coll. W. T. Blanford, Esq.

\section{var. latefasciata.}

16 Mauritius ; coll. G. Nevill, Esq.

subvar. minor.

5 Bombay; coll. W. T. Blanford, Esq.

9. Fenella [?] sp.

1 Hongkong; coll. R. Hungerford, Esq.

10. Fenella n. sp. ?

3 Bombay; coll. W. T. Blanford, Esq.

\section{Fenella fulgida [?].}

Rissoa fulgida, C. B. Adams [ubi ?].

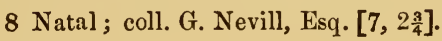

Identified for me as $F$. fulgida, C. B. Adams, by Herr Schwartz von Mohrenstern.

var. [? dist. sp.].

3 Natal; coll. G. Nevill, Esq.

var. [? dist. sp.].

8 Mauritius; coll. G. Nevill, Esq.

12. Fenella sp.?

2 Seycheiles ; coll. G. Nevill, Esq. 
var. [? dist. sp.].

2 Singapore; coll. Dr. F. Stoliczka.

\section{Fenella reticulata.}

Dunkeria reticulata, A. Adams, Ann. Mag. VI, 1860. p. 422, Awa-Sima,

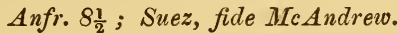

3 Japan; ex Mr. Damon [“coll. A. Adams"].

Long. $3 \frac{1}{2}$, diam. $1 \frac{1}{6}$ mil.

var. ?

5 Henjam [Persian Gulf] and Annesley Bay; coll. W. T. Blanford, Esq.

4. Nicobars ; coll. I)r. F. Stoliczka.

All in very bad condition.

\section{Fenella vinacea.}

Rissoa vinacea, Recluz [ubi ?].

20 Mauritius; coll. G. Nevill, Esq.

Identified for me, as above, by Herr G. Schwartz von Mohrenstern.

\section{Fenella pupoides.}

Finella pupoides, A. Adams, Ann. Mag, VI, 1860, Tsu-sima and Korea Strait; J. de C., 1S68, pl. IV, fig. 5; Samoa, fide Schmeltz, and Suez, fide McAndrew; $?=R$. virgata, Phil.

4 Japan; ex Mr. Damon [c. A. Adams] and Dr. F. Stoliczka.

Long. $3 \frac{3}{4}$, diam. $1 \frac{1}{4}$ mil.

3 Hongkong; coll. R. Hungerford, Esq.

1 Natal ; coll. G. Nevill, Esq. [identified by Herr Schwartz]

4 Aden and Nicobars; coll. Dr. F. Stoliczka.

6 New Caledonia; ex c. P. Bouvier, Esq.

2 Mauritius; coll. G. Nevill, Esq.

\section{var. fusco-apicata [? dist. sp.]}

20 Bombay ; coll. W. T. Blinford, Esq. 
16. Fenella infratincta [?].

Fenella infratincta, Garrett, P. Acad. Philad., 1873, Viti and Samoa 1sl. long. $2 \frac{1}{2}$ mil.

2 Samoa ; ex Mus. Godeffroy [long. $3 \frac{7}{2}$ mil.; labelled " $F$. pupoides"].

17. Fenella u. sp.

12 Mauritius; coll. J. B. Baxter and G. Nevill, Esqrs.

18. Fenella n. sp.

2 Tawila [Persian Gulf]; coll. W. T. Blanford, Esq.

19. Fenella n. sp.

2 Tawila [Persian Gulf]; coll. W. T. Blanford, Esq.

Something like $F$. rufocincta, A. Adams.

20. Fenella sp.

2 Tawila [Persian Gulf]; coll. W. T. Blanford, Esq.

\section{Fenella fusca.}

Dunkeria fusca, A. Adams, Ann. Mag. VI, 1860, Tabu-Sima, 25 fths. Anfr. $8 \frac{1}{2}$ [emend. 1864].

2 Japan; ex Mr. Damon [" coll. A. Adams"].

Long. $5 \frac{1}{2}$, diam. $1 \frac{9}{10} \mathrm{mil}$.

22. Fenella craticulata.

unkeria craticulata, A. Adams, Ann. Mag. 1860, Gotto I. and Mino-Sima.

1 Japan; ex Mr. Damon ["coll. A. Adams"]

23. Fenella n. sp.

20 Tawila, Persian Gulf; coll. W. T. Blanford, Esq. 
Something like $F$. rufocincta, A. Adams, but smaller, with different sculpture, \&c.

\section{Fenella sp.}

6 Hongkong; coll. R. Hungerford, Esq.

25. Fenella n. sp.

1 Ceylon; coll. G. Nevill, Esq.

26. Fenella [?] sp.

1 Hongkong; coll. R. Hungerford, Esq.

Presents some of the characters of Turbonilla.

Genus ONOBA, H. \&. Adams.

Gen. Moll. I, 185̃, type Turbo striatus, J. Adams.; sect. ?=Bacula, H. and A. Adams, Ann. Mag. XI, $1863 ;=$ Pelycidium, Fischer, MIS.

Other sp. of the Genus from Eastern Seas are Rissoa hyalina [6 mil.] and R. truncata [2 mil.], Garrett, P. Ac. Phil., 1873, Fiji Isl.

Three sp. have been described by A. Adams from Japan, Ann. Mag. 1861 and 1863; one is found also at Suez, Ann. Mag. 1870. The R. striatula, Pease, A. J. C., 1868, Paumotus, 9, $3 \frac{1}{2}$-perhaps belongs here, or to Bacula, Ad.?

\section{Onoba striata.}

Turbo striatus, J. Adams, Tr. Linn. Soc. III, 1800; fide Jeffreys, vars. $=$ Rissoa arctica, Loven, and =R. saxatilis, Möller.

10 England; coll. G. Nevill, Esq.

var. saxatilis.

Rissoa saxatilis, Möller, Ind. Möll. Grönland, 1842.

1 Hammerfest; ex "Linnæa," 
2. Onoba n. sp.

20 Arakan and Singapore; coll. Dr. F. Stoliczlsa.

var. [? dist. sp.].

I Arakan; coll. Dr. F. Stoliczka.

\section{Onoba n. sp.}

2 Singapore; coll. Dr. F. Stoliczka.

\section{Onoba n. sp.}

1 Andamans; coll. J. Wood-Mason, Esq.

\section{Onoba delicata.}

Rissoa delicata, Philippi, Zeits. Mal., 1849, p. 34, Aden, long. $1 \frac{1}{3}$.

2 Bombay; coll. Rev. S. B. Fairbank [5, 1\$?],

1 Roweiah, Red Sea; coll. J. B. Baxter, Esq. $\left[6 \frac{3}{4}, 2 \frac{3}{4}\right]$.

4 Ceylon; coll. G. Nevill, Esq.

These Herr Schwartz identified as O.pulchella, C. B. Adams.

1 Aden; coll. G. Nevill, Esq. $\left[4 \frac{5}{2}, 1 \frac{3}{4}\right]$.

var. microstoma, nov.

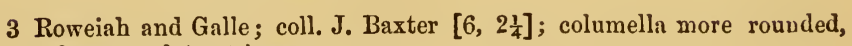
less angulate at base.

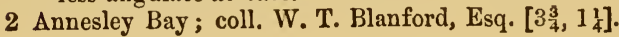

6. Onoba n. sp.

Anfr. $4 \frac{1}{2}$; long. 3, diam. 1 mil.

3 Tumb Island, and Bombay; coll. W. T. Blanford, Esq.

7. Onoba [?] n. sp.

20 Singapore and Malacca; coll. Dr. F. Stoliczka.

8. Onoba n. sp.

12 Calicut; coll. Dr. F. Stoliczka. 


\section{Onoba n. sp.}

Long. 1 mil. [vixj; anfr. $3 \frac{1}{2}$.

2 Andamans; coll. G. Nevill, Esq.

var. [? dist. sp.].

2 Andamans; coll. G. Nevill, Esq.

10. Onoba sp.

1 Hongkong; coll. R. Hungerford, Esq.

There is another sp. of Onoba, said to be from Hongkong and Senegal [!] figured by Folin, Fonds de la Mer, Pl. VIII, fig. 5, as Pelycidium venustulum.

Sub-Genus CERATIA, H. \& A. Adams.

Gen. Moll. I, 1854, type Rissoa proxima, Alder.

There is a C. pyrgula, A. Adams, from Suez, Ann. Mag. 1870, also a species from New Guinea described by Tapparone-Canefri.

\section{Onoba (Ceratia) proxima.}

Rissoa proxima, Alder, Forbes and Hanley, Brit. Con. III, $1853 ;=\boldsymbol{R}$. striatula, Jeffr., Ann. MIag., 1858, not Turbo (= Rissoa) striatulus, Lin.

1 England; ex c. G. Nevill, Esq.

1 Algiers; coll. P. Joly, Esq. ("very rare").

Sub-Genus HYALA, H. \& A. Adams.

Gen. Moll. I, 1854, type Turbo vitreus, Mtg.

Three species are described by A. Adams, Ann. Mag., 1870, from Suez, as belonging to this Genus. Rissopsis, Garrett, appears to be closely allied.

\section{Onoba (Hyala) vitrea.}

Turbo vitreus, Montagu, Test. Brit., 1803.

2 England; ex c. G. Nevill, Esq.

6 Algiers; coll. P. Joly, Esq. 
13. Onoba (Hyala) nitida.

Hyala nitidu, A. Adams, Ann. Mag., 1870, Suez; anfr. 6.

2 Roweiah, Red Sea; coll. J. B. Baxter, Esq.

var. minor.

1 Roweiah; coll. J. B. Baxter, Esq.

14. Onoba (Hyala) n. sp.

2 Mauritius and Seychelles; coll. G. Nevill, Lisq.

15. Onoba (Hyala) sp.

2 Hongkong; coll. R. Hungerford, Ess.

var.

1 Mauritius; coll. J. B. Baxter, Esq.

16. Onoba (Hyala) n. sp.

16 Mauritius and Seychelles; coll. G. Nevill, Esq.

var. ( ? )

4. Persian Gulf; ex c. Coloncl G. B. Mainwaring.

17. Onoba (Hyala) sp.

2 Mauritius; coll. J. B. Baxter, Esq.

18. Onoba (Hyala) sp.

1 Hongkong; coll. R. Hungerford, Esq.

19. Onoba (Hyala) n. sp.

3 Mauritius; ex c. Dr. F. Stoliczka.

var. ?

1 Mauritius; coll. J. B. Baxter, Esq- 
20. Onoba (Hyala) pumilio.

Hyala pumilio, A. Adams (ubi?).

1 Jap̧an; ex Mr. Damon (" coll. $\Lambda$. Adauns").

21. Onoba (Hyala) glabrata.

Hyali glabrata, Philippi, fide A. Adlams ms.

1 Jupan; ex Mr. Danon (" coll. A. Adauns"),

22. Onoba (Hyala ?) sp.

1 Arakan; coll. Mr. F. Stoliczka.

23. Onoba (Hyala) n. sp.

1 Munitius; ex c. Dr. F. Stoliczka.

A form with two transverse bands.

24. Onoba (Hyala) sp.

2 Hongkong; coll. k. Hungerfork, Hsq.

var. minor.

2 IIongkong; coll. R. Hungerford, Esq.

Genus AMPHithalmus, Carpenter.

Ann. Mag., XT, 1S65, type A. inclusus, Carp.

\section{Amphithalmus inclusus.}

Amphithalmus inclusus, Carpenter, Ann. Mrag., $\boldsymbol{X} \nu, 1865$, California.

1 California; ex Mr. Damon.

\section{Amphithalmus pupoides.}

Amphithahmus pupoides, H. Adams, P. Zool. S., 1865, Lord IIood's Island1순, $\frac{1}{2}$ mil.

1 Lord Hood's I. (?); ex Mr. Damon. 
H. Adams remarks on its resemblance to Stenothyra, \&c.; Barleeia? microthyra, Martens, from Mauritius, also belongs here; possibly, however, they may both, as well as $A$. obesus and $A$. pellyce prove generically separable from the typical Cali. fornian species?

\section{Amphithalmus obesus.}

Amphithalmus obesus, H. Adams, P. Zool. S., 1865, Lord Hood's Islandlong. $1 \frac{3}{4}$, diam. $\frac{1}{2}$ mil.

1 Lord Hood's I. (?); ex Mr. Damon.

\section{Amphithalmus pellyæ.}

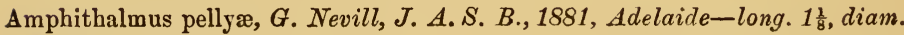
$\frac{1}{2}$ mil.

1 Adelaide (type); coll. Miss Pelly.

\section{Genus CINGULA, Fleming.}

Hist. Brit. Animals, 1828, type Turbo cingillus, Mtg.; sect. = Peringilla, Monterosato.

\section{Cingula cingillus.}

Turbo cingillus, Montagu, Test. Brit., 1803 ; var. rupestris, Forb., Ann. Mag. V-“bandless."

2 Weymouth; coll. G. Nevill, Esq.

\section{Cingula glabrata.}

Turbo glabratus, Mühlfeld, Terh. Ges. Nat. Fr. Berlin, 1818 and 1829.

20 Algiers; coll. P. Joly, Esq.

3. Cingula (?) nigra.

Assiminea nigra, Krauss, Sïd-Afrik. Moll. 184S, Alyoa and Table Bayslong. 0.7, diam. $0 \cdot 4$ lin.

var. (?)

1 Port Natal; coll. G. Nevill, Esq. 
4. Cingula (?) sp.

1 Port Natal; coll. G. Nevill, Esq.

This may perhaps be a species of the Hydrobiina.

\section{Cingula (?) sp.}

1 Mauritius; coll. G. Nevill, Esq.

\section{Cingula n. sp.}

\& Mauritius ; coll. G. Nevill, Esq.

7. Cingula n. sp.

50 Annesley Bay, Red Sea; coll. W. T. Blanford, Esq.

A pretty little species, only 1 mil. in length!

8. Cingula (?) n. sp.

5 Annesley Bay; coll. W. T. Blanford, Esq.

\section{Cingula tiberiana.}

Cingula tiberiana, Issel, Malac. Mar Rosso, 1869, Suez-long. 1 $\frac{1}{2}$, diam. 1 mil., and Descr. de l'Egypte, pl. III, fig 16.

5 Annesley Bay; coll. W. T. Blanfurd, Esq.

1 Mauritius; coll. G. Nevill, Esq.

\section{Cingula villæ (?)}

Cingula villæ, Issel, Malac. Mar Rosso, 1869, Suez-long. $2 \frac{1}{2}$, diam. $1 \frac{1}{3}$ mil. and Desc. de l' Egypte, pl. III, fig. 17.

20 Singapore? (or Arakan ?); coll. Dr. F. Stoliczka.

Long. $2 \frac{3}{4}$, diam. $1 \frac{1}{8}$ mil., scarcely rimate.

var.?

Singapore; coll. Dr. F. Stoliczka.

\section{Cingula semistriata.}

Turbo semistriatus, Montagu, Test. Brit. Sup. 1808 ; not Rissoa semistriata, Philippi (Alaba sp.); = R. subsulcata, Philippi, Sicily.

20 Algiers; coll. P. Joly, Esq. 
12. Cingula [?] madreporica.

Cingula madreporica, Issel, Malac. Mar Rosso, 1869, Suez.

Long. $1 \frac{1}{3}$, diam. $\frac{1}{3}$ mil.

2 Annesley Bay; coll. W. T. Blanford, Esq.

13. Cingula [?] n. sp.

1 Aden; coll. Dr. F. Stoliezka.

Perhaps rather a species of Setia?

14. Cingula seminulum.

Rissoa seminulum, Monterosato, J. de C., 1877, Sidi Ferruch, pl. III, fig. 8.

8 Algiers; coll. P. Joly, Esq.

15. Cingula (?) sp.

3 Andamans and Mauritius; coll. G. Nevill, Esq.

\section{Cingula intorta.}

Rissoa intorta, Monterosato, Enum. Conch. Medit., 1872, for Ris.soa contorta, Jeffreys, var. intorta, Monterosato, Ann. Mus. Civ. Genova, 1876.77, Civita-vecchia.

4 Algiers; coll. P. Joly, Esq.

17. Cingula lævis.

Rissoa lævis, Monterosato, J. de C., 1877, Algiers, coll. Joly, (not C. lavis, Dekay); sect. Peringiella.

20 Algiers (typical); coll. P. Joly, Esq.

[?] Sub-Genus PUTILLA, A. Adams.

P. Zool. S., 1867, type P. lucida, A. Adams.

Putilla has been referred, possibly correctly, to the family Eulimidoe.

The three species described by A. Adams, Ann. Mag. VI, 1870, from Suez, as "Rissoella sp." seem to me to belong to this group? 
18. Cingula ? (Putilla) n. sp.

1 Henjam, Persian Gulf ; coll. W. T. Blanford, Esq.

19. Cingula ? (Putilla) n. sp.

10 Oman, Persian Gulf ; coll. W. T. Blanford, Esq.

20. Cingula ? (Putilla) lucida.

Putilla lucida, A. Adams, P. Zool, S. 186\%, Gotto.

1 Japan ; ex Mr. Damon ["coll. A. Adams"]

Gends [?] SETIA, H. \&. A. Adams.

Gen. Moll. I, 1854, type S. puulcherrina, Jeffr.

From the characters of the shell alone, I am unable to detect distinctive differences to separate Setia from Cingula [or Barleeia?].

\section{Setia candida.}

Setia candida, A. Adams, Ann. Mag. VIII, 1861, Sado Isl.

1 Japan ; ex Mr. Damon ["coll. A. Adams"].

\section{Setia pulcherrima.}

Rissoa pulcherrima, Jeffreys, Ann. Mag., 184S, Ser. 2, Tol. III, Channel Islands.

8 England: ex Mr. Damon.

\section{Setia fulgida.}

Helix fulgida, J. Adams, Trans. Lin. Soc., III, 1800, England $=R$. pygmaa, Michaud, fide Weinkauff.

1 Loc. ? ex Mr. R. Damon ["coll. A. Adams"].

\section{Setia soluta.}

Rissoa soluta, Philippi, Moll. Sicil. 1836.

1 Exmouth; ex "Linnæa." 
5. Setia n. sp.

1 Andamans; coll. G. Nevill, Esq.

6. Setia n. sp.

4 Mauritius; coll. G. Nevill, Esq.

7. Setia [? ?] n. sp.

1 Natal ; coll. G. Nevill, Esq.

8. Setia [?] bizonata, nov.

Long. $1 \frac{1}{2}$, diam. $\frac{1}{2}$ mil.; anfr. 4 .

2 Mauritius [type]; coll. G. Nevill, Esq.

May possibly prove the S. bifasciata of A. Adams-Ann. Mag., VIII, 1861, Japan-with the very meagre description of which it agrees.

var. major [? dist. sp.].

1 Mauritius; coll. G. Nevill, Esq.

9. Setia bifasciata [?].

Setia bifasciata, A. Adams, Ann. Mag. VIII,1861, Awa-Sima.

1 Japan (?); ex Mr. Damon [“ coll. A. Adams"].

This is certainly not S. pulcherrima, Jeffreys, although sent me as "Setia pulcherrima, A. Adams, from Japan"; it is a larger form than the preceding, to which it is, however, closely allied.

10. Setia n. sp.

3 Adelaide; coll. Miss Pelly.

With two brown bands on the base.

11. Setia n. sp.

3 Adelaide; coll. Miss Pelly.

A small, pure white species. 
RISSOID赵.

12. Setia [?] n. sp.

1 Adelaide; coll. Miss Pelly.

\section{Setia fusca.}

Rissoa fusca, Philippi, Enum. Moll. Sicil. 1836.

3 Palermo; ex c. P. Joly, Esq.

12 Algiers; coll. P. Joly, Esq.

14. Setia [?] n. sp.

1 Adelaide; coll. Miss Pelly. An umbilicated form.

Genus NEVILLIA, $H$. Adams.

P. Zool. S. 1868, p. 289, type N. picta, H. Adams [not Nevillia of Mar. tens].

\section{Nevillia picta.}

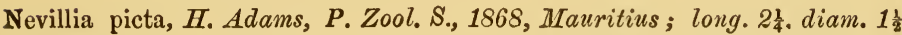
mil.

12 Mauritius [type]; coll. G. Nevill, Esq.

Very variable and beautiful in its coloration; some are of a uniform dark brown, some uniform crimson, bright pink variegated with white, or white variegated with pink, \&c.

\section{Nevillia lucida.}

Nevillia lucida, H. Adams, P. Zool. S. 1868, Bourbon; long. 2, diam. $1 \frac{1}{2}$ mil.

1 Bourbon [type]; coll. G. Nevill, Esq.

8 Mauritius; coll. G. Nevill, Esq.

\section{Gends EATONIELLA, Dall.}

Bull. U. S. Mus.1876, for Eatonia, Smith, Ann. Mag., XVI, 1870, type B. kerguelensis, Smith, not Eatonia, Hall, 1859, [Gen. Brachiopoda.]

Not in the Museum Coll.

Genus CROSsEA, A. Adams.

Ann. Mag. 1865, type C. miranda, A. Adains; Crosse, J. de C., 1868, pl. IV, figs. 9-10. 
1. Crossea [?] n. sp.

1 Andamans; coll. J. Wood-Mason, Esq.

2. Crossea [?] n. sp.

1 Annesley Bay, Red Sea; coll. W. T. Blanford, Esq.

A very lovely, small form. Can it possibly be the Corena tuberculifera, A. Adams, from Suez?

\section{Crossea concinna.}

Crossea concinna, Angas, P. Z. S. Pt. Jackson.

1 Loc.?; ex Mr. Damon ["coll. A. Adams"]

Alt. $2 \frac{3}{4}$, diam. $3 \frac{3}{4}$ mil.

10 Pt. Jackson; coll. G. Nevill, Esq.

\section{Crossea bellula.}

Crossea bellula, A. Adams, Ann. Mag. 1865, Gotto Isl.; Crosse, J. de C., 1863, pl. IV, fig. 10.

1 Japan; ex Mr. Damon ["coll. A. Adams"].

Alt. $1 \frac{3}{4}$, diam. $1 \frac{3}{4}$ mil.

\section{Genus AnABATHRon, Frauenfeld.}

Moll. Novara Exp., 1866, type A. contabulata, Fr. from Botany Bay.

\section{Anabathron contabulata.}

Anabathron contabulata, von Frauenfeld, Novara Moll. 1866, Botany Bay.

30 Botany Bay; coll. G. Nevill, Esq. [several vars.].

\section{Genus SCALIOLA, A. Adams.}

Ann. Mag. VI, 1860, type S. bella, A. Adams, as Sub-Gen. of Scala; emend., l.c., $X, 1862$, as Genus of Rissoida.

\section{Scaliola bella.}

Scaliola bella, A. Adams, Ann. Mag. VI, 1860, and X, 1862, Tabu-Sima, 25 fths.

Long. $3 \frac{1}{4}$, diam. $1 \frac{3}{4}$ mil.

1 Japan [typical]; ex Mr. Damon ["coll. A. Adams"]. 
2. Scaliola, n. sp.

16 Mauritius ; coll. G. Nevill, Esq.

3. Scaliola, n. sp.

6 Andamans; coll. G. Nevill, Esq.

1 Seychelles; coll. G. Nevill, Esq.

var. [?]

3 Mauritius; coll. G. Nevill, Esq.

\section{Scaliola gracilis.}

Scaliola gracilis, A. Adams, Ann. Mag. X, 1862, Gotto, 71 fths.

Long. 2, diam. $\frac{9}{10}$ mil.

1 Japan [typical]; ex Mr. Damon [“coll. A. Adams"].

var. [?]

2 Andamans; coll. G. Nevill, Esq.

\section{Scaliola arenosa.}

Scaliola arenosa, A. Adams, Ann. Mag. X, 1862, Osima, Tanabe and Simoda.

Long. 3, diam. 1 mil.

2 Japan [typical]; ex Mr. Damon ["coll. A. Adams"].

50 Seychelles and Mauritius; coll. G. Nevill, Esq.

They agree well with the typical specimen. Herr Schwartz also identified them as $S$. arenosa.

1 Japan [long. 3, diam. 1 mil.]; ex Mr. Damon [labelled "S. lacrenosu, A. Adams "].

1 Singapore; coll. Dr, F. Stoliczka.

10 Bombay; coll. W. T. Blanford, Esq.

\section{Scaliola glareosa.}

Scaliola glareosa, A. Adams, Ann. Mag. 1862, Gotto Isl., $71 \mathrm{fths}$.

Long. $2 \frac{3}{4}$, diam. $\frac{9}{10}$ mil. 
2 Japan [typical]; ex Mr. Damon [“coll. A. Adams "].

40 Singapore; coll. Dr. F. Stoliczka.

10 Mauritius; coll. G. Nevill, Esq.

18 Andamans and Nicobars; coll. J. Wood-Mason, Esq., and Dr. F. Stoliczka.

3 Hongkong; coll. R. Hungerford, Esq.

\section{Scaliola elata.}

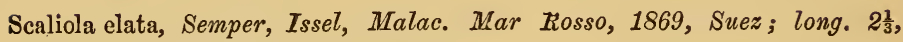
diam. 1 mil., and Zanzibar [Semp.].

Seems to me doubtfully distinct from $S$. arenosa?

5 Suez [typical]; coll. Prof. A. Issel.

50 Annesley Bay; coll. W. T. Blanford, Esq.

var. [?]

2 Aden; coll. Dr. F. Stoliczka.

\section{Scaliola, n. sp.}

1 Nicobars; coll. Dr. F. Stoliczka.

\section{[?] Genos MiCROSTELma, A. Adams.}

Ann. Mag. XI, 1863, type M. daedala, A. Ad., Gotto Isl.; M. concinna, A. Adams, l. c., VI, 1870, from Suez, fide McAndrews, a broken Rissoina!

This and the two next Genera are not represented in the Museum.

[?] Genus CORENA, A. Adams.

Ann. Mag. VI, p. 122, 1870, type C. tuberculifera, A. Ad.; from Suez.

[?] Genus RISSOPSIS, Garrett.

P. Acall. Philad. 1873, type R. typica, Garrett, from Viti and Samoa. 


\section{Family HETEROPHROSYNID王, Clarke.}

\section{Genus JeFFREYSIA, Alder.}

Forbes and Hanley, Brit. Conch. 1849, and Alder, Ann. Mag. TII, 1851, type R. [?] diaphana, Ald.; fide Jeffreys $=$ Rissoella, Auct. [not of Gray, type of which is Odostomia glabra, Brown].

There are several other species described by A. Adams, Ann. Mag. 1860 and 1861, from Japan and China.

\section{Jeffreysia diaphana.}

Rissoa? diaphana, Alder, Ann. Mag. XIII, 1844, England; fide Wein. kauff, should be J. glabra, Brown.

10 England; ex G. Nevill, Esq.

\section{Jeffreysia [?] minima.}

Jeffreysia minima, A. Adams, Ann. Mag. VI, 1860, Tsu-Sima, at 26 fths.

Long. [vix] 1 mil.

1 Japan; ex Mr. Damon ["coll. A. Adams "].

\section{Jeffreysia [?] mundula.}

Jeffreysia mundula, A. Adams, Ann. Mag. VI, 1860, Tsu.Sima at 26 flhs.

Even smaller than the preceding!

1 Japan; ex Mr. Damon ["coll. A. Adams"].

\section{Jeffreysia [?] sp. ?}

1 Japan; ex Mr. Damon ["coll. A. Adams"].

There are no bands on this specimen. It was sent me labelled "Rissoella bifasciata," Carpenter.

\section{Jeffreysia [?] sp. ?}

3 Japan ex Mr. Damon ["coll. A. Adams"]. 
There are traces of two bands on these specimens. Received as "Rissoella fulgida, A. Ad." !

\section{Jeffreysia [?] turgidula [?]}

Jeffreysia (?) turgidula, A. Adams, Ann. Mag. VI, 1860, Korea Strait, at 46 fths.

1 Japan; ex Mr. Damon ["coll. A. Adams"].

Received as "Rissoella miridula, A. Ad."

\section{Jeffreysia [?] sp.}

1 Japan; ex Mr. Damon ["coll. A. Adams"].

Received as "Rissoella glabra, A. Ad."

\section{Gendos BARLEEIA, Clark.}

Brit. Marine Test. Moll. 1855, type Turbo ruber, Mtg.

\section{Barleeia rubra.}

Turbo ruber, Montagu, Test. Brit. 1803; not Turbo ruber, Adams [ $R$. parva, var. fide Jeffr.].

20 England, \&c.; ex c. Dr. F. Stoliczka, and G. Nevill, Esq.

\section{var. major.}

Barleeia rubra, var. major, Monterosato, J. de C., 187\%, Algiers.

30 Algiers; coll. P. Joly and G. Nevill, Esqrs.

\section{var. unifasciata.}

Turbo unifasciatus, Montagu, Test. Brit. 1803.

1 Falmouth; ex "Linnæa."

var. pallida.

Barleeia rubra, var. pallida, Jeffreys, Brit. Con. IlI.

1 Algeria; ex “Linnæa." 


\section{Family LITTORINIDÆ, Gray.}

\section{Genus LITTORINA, Férussac.}

Tabl. Syst. 1821, type L. litorea, Lin.; sect.=Neritoides, Brown, type L. obtusata, Lin.; sect.=Melaraphe, Mühlfeld, type L. neritoides, Lin. ; sect. = Littorinopsis, Mörch, Mal. Blät. 1876, type L. ungulifera, Lamarck.

\section{Littorina littorea.}

Turbo littoreus, Linnaus, Syst. Nat. X, 1758, Norway; Jeffreys, 1865, vars. paupercula, brevicula, turrita and sinistrorsa.

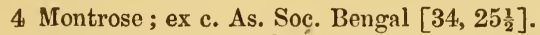

10 England, Dunkirk, \&c.; ex c. Dr. F. Stoliczka.

2 N. France; coll. P. Bouvier, Esq.

\section{Littorina rudis.}

Turbo rudis, Maton, Linn. Soc. Trans. VIII, 1807; fide Jeffreys, vars. =L. saxatilis, Johnston =Turbo sulcatus, Leach =Turbo jugosus, Mtg. and vars. globosa, similis, lavis and compressa, also $=L$. sitchensis, Phil. $=$ L. groenlandica, Menke.

20 Dunkerque, England, \&c.; ex c. Dr. F. Stoliczka, \&c.

1 Port Said; coll. J. B. Baxter, Esq.

var. saxatilis.

Littorina saxatilis, Johnston, Trans. Berwick Soc. 1838.

1 Tilbury; ex "Linnæa."

var. patula.

Littorina patula, Jeffreys, Thorpe, Brit. Mar. Conch., 1844.

50 United States; ex c. Prof. W. Newcomb, \&c.

var. tenebrosa.

Turbo tenebrosus, Montagu, Test. Brit. II, 1803 ; var. of L. rudis, fide Jeffreys.

50 United States; coll. Prof. E. C. Stearns, \&c.

4 Prince Edward's Island; coll. G. Nevill, Esq. 


\section{Littorina obtusata.}

Turbo obtusatus, Linnceus, Syst. Nat. X, 1758, North Sea; fide Jeffreys, vars. Turbo fabalis, Turton, ornata and compacta; sect. Neritoides.

12 Dunkerque, England, \&c. ; coll. Dr. F. Stoliczka, \&c.

6 Isle of Wight; coll. G. Nevill, Esq.

var. neritiformis.

Littorina neritiforma, Brown, Ill. Conch. Gt. Brituin, 1845.

18 England; ex c. G. Nevill, Esq., and As. Soc., Bengal.

var. limata.

Littorina obtusata, var. limata, Löven.

1 Hammerfest; ex "Linnæa."

\section{Littorina sitchensis.}

Littorina sitkana, Philippi, P. Zool. S. 1845, emend. Abb., pl. TI, figs. 13 and 18, Sitcha; fide Jeffreys $=$ L. rudis, var.

10 Vancouver Isl., Kadiak, \&c.; coll. Profs. W. Newcomb and E. C. Stearns.

subvar. sublævis.

4 Sitka, \&c.; coll. Profs. W. Newcomb and E. C. Stearns.

subvar. fasciata.

1 Kadiak; coll. Prof. E. C. Stearns.

\section{Littorina scutulata.}

Littorina scutulata, Gould, P. Bost. S., 1849, Puget Sound.

12 Monterey and Purissima, California; coll. Prof. E. C. Stearns.

\section{Littorina neritoides.}

Turbo neritoides, Linnceus, Syst. Nat. X, 1758, Mediterranean; fide Jeffrey $s=L$. basterotii, Payr., \&c.; sect. Melaraphe.

10 Algiers; coll. P. Joly and G. Nevill, Esqrs.

1 Menton [? sub-fossil] ; coll. G. Nevill, Esq.

10 Dalmatia ; ex c. Dr. T. Oldham [labelled "Meluphre glabrata"].

10 Italy and Trieste; ex c. Dr. F. Stoliczka, \&c. 


\section{Littorina punctata.}

Turbo punctatus, Gmelin, Syst. Nat. 1790, Senegal; sect. Melaraphe.

10 Algiers; coll. P. Joly, Esq.

var. syriaca.

Littorina syriaca, Philippi, Abb. II, 184\%, Syria [bene].

1 Beirut; coll. Dr. J. Anderson.

A very distinct form not recorded by Reeve, but scarcely specifically distinguishable?

\section{Littorina palliata.}

Turbo palliatus, Say, J. Acad. Philad. II, 1818, Uuited States; fide Jeffreys =Nerita littoralis, Linn.; sect. Neritoides.

20 Long Island, \&c. ; coll. Rev. E. R. Beadle, \&c.

8 Prince Edward's Island; coll. A. Nevill, Esq.

\section{Littorina grœnlandica.}

Littorina grœnlandica, Menke, Syn. Moll., 1830, Greenland; fide Jeffreys= L. rudis, var.

5 Greenland; ex c. G. Nevill, Esq.

10. Littorina africana.

Littorina africana, Krauss, Phillippi, Abb. II, 1847, Algoa Bay; sect. Melaraphe.

7 Port Natal ; coll. G. Nevill, Esq., \&c.

10 Table Bay [Cape Town]; ex c. Dr. F. Stoliczka.

\section{Littorina brevicula.}

Turbo breviculus, Philippi, Zeits. Mal., 1844, Emb. Yangtsekiang var. $=L$. souverbiena, Crosse.

5 Kulangsu (Amoy); coll. R. Hungerford, Esq.

2 Nagasaki ; coll. R. Hungerford, Esq. 


\section{Littorina porcata.}

Littorina porcata, Philippi, Proc. Zool. Soc., 1845, Galapagos.

5 Panama; ex c. Prof. W. Newcomb.

\section{Littorina planaxis.}

Littorina planaxis, Nuttall MS., Phitippi, Abb. II, 184\%, pl. IV, fig. 16, Upper California; sect. Melaraphe.

12 Bodega, and Monterey [California]; coll. Prof. E. C. Stearns.

\section{Littorina aspera.}

Littorina aspera, Philippi, P. Zool. S. 1845 and Abb. II, 1847, pl. IV, fig. 13, "Sitcha, Mexico, and S. Salvador"; ? sect. Littorinopsis.

12 Panama; coll. Prof. E. C. Stearns.

\section{Littorina conspersa.}

Littorina conspersa, Philippi, Abb. II, 1847, pl.IV, fig. 14, Real Llejos: $?$ sect. Littorinopsis.

10 Nicaragua ; coll. Prof. E. C. Stearns.

var. fortisulcata.

10 Nicaragua; coll. Prof. E. C. Stearns.

\section{Littorina minima.}

Turbo minimus, Gray, Wood's Sup. Index Test., 1828, Hab. ?; sect. Mela. raphe.

10 W. Indies; ex c. Prof. W. Newcomb.

\section{Littorina lineata.}

Littorina lineata, Lamarck, Hist. VII, 1822, Hab.?; fide Mörch = L. tessellata, Phil. 1847; sect. Littorinopsis.

5 West Indies; ex c. Prof. W. Newcomb.

1 Guadaloupe; ex c. P. Joly, Esq. 
18. Iittorina mespilum.

Helix mespilum, v. Mühlfeld, Verh. Berlin Ges., I, 1829, Antilles; $=L$. fusca, Pfr. 1840, Cuba; =L. naticoides, Orb.; sect. Melaraphe.

2 Jamaica; ex c. Prof. W. Newcomb.

\section{Littorina plena.}

Littorina plena, Gould, P. Bost. S. 1849, S. Francisco, long. $\frac{1}{4}$, lat $\frac{1}{5}$ poll. and Expl. Exp., pl. 14, fig. 236; ? sect. Melaraphe.

var. vitiensis.

Littorina vitiensis, Dunker.

3 Tahiti; ex Mus. Godeffroy.

Long. $8 \frac{1}{2}$, diam. $6 \frac{1}{4}$ mil. I am by no means sure that this form is Gould's L. plena. The spire is very much shorter, \&c.

\section{Littorina picta.}

Littorina picta, Philippi, Abb. II, 184\%, pl. III, fig. 26, Sandwich Islands and Luzon, with var. marmorata.

12 Sandwich Islands; coll. H. Pease, Esq., and Prof. W. Newcomb.

\section{Littorina ziczak.}

Trochus ziczac, Chemnitz, Conch. Cab. I, Vol. $\nabla$, fig. 1599; fide Mörch $=L$. orbignyana, Philippi, Abb. III; sect. Melaraphe.

7 Guadaloupe; ex c. Prof. W. Newcomb and P. Joly, Esq.

10 Aspinwall; ex c. Prof. E. C. Stearns.

\section{Littorina philippii.}

Littorina philippii, Carpenter, Cat. Mazatlan Shells, $185 \%$.

30 Gulf of California; ex c. Profs. W. Newcomb and F. C, Stearns.

Has been stated to be the Lit. dubiosa of C. B. Adams. 


\section{Littorina angustior.}

Littorina angustior, Mörch, Malak. Blät. XXIII, 18\%6, Havanna, \&c., for, Orbigny's Moll. Cuba, pl. 14, figs. 24 and 26, as "L. lineata"; =L carinata, Reeve [as of Sow.], fig. 50, Cuba, \&c.; var. fasciata, Mörch l. c., Orbigny's Cuba, pl. 14, fig. 25, ; sect. Melaraphe.

10 Cuba; ex c. Prof. W. Newcomb.

3 Guadaloupe; $\theta x$ c. P. Joly, Esq.

\section{Littorina zebra.}

Turbo zebra, Gray, Wood's Sup. Index Test. 1828, S. America; sect. Melaraphe.

8 Valparaiso; coll. Prof. E. C. Stearns.

var. nana.

Long. 10, diam. $6 \frac{1}{2}$ mil.

2 Valparaiso; coll. Prof. E. C. Stearns.

\section{Littorina pintado.}

Turbo pintado, Gray, Wood's Sup. Index Test. 1828, Sandwich Islands; fide Pease, Am. J. Con. IV,=L. ambigua, Phil.=L. serialis, Soul. $=L$. tenebrata, Nuttall; ? sect. Melaraphe.

9 Sandwich Islands; ex c. Prof. W. Newcomb.

Fide v. Martens from Mauritius and $=L$. serialis. I cannot but think, however, this must be merely a form of $L$. glabrata [my L. obesa, var. glabrata]. I have never seen Mascarene specimens that agree with those of the Sandwich Isl.; though indeed the two species are perhaps scarcely more than geographical races? Three of the above agree with Reeve's fig. of $L$. tenebrata, probably the female form.

\section{var. serialis.}

Littorina serialis, Souleyet, Voy. Bonite, 1852, pl. XXXI, figs. 34-36, Sandwich Islands.

3 Sandwich Islands; ex c. Prof. W. Newcomb [as L. serialis].

A rather distinct form, does not agree well with Souleyet's figure. 


\section{Littorina diemenensis.}

Littorina diemenensis, Quoy, Voy. Astrolabe, II, 1839, Tasmania and New Zealand; Philippi, II, pl. IV, fig. 1 [bene]; Reeve, fig. 94 [male]; sect. Melaraphe.

8 Sydney; ex c. Dr. F. Stoliczka.

\section{var. pseudolævis.}

Reeve, Con. Ic. fig. 95, as L. lavis [not L. lavis, Phil.]

6 New South Wales; ex c. Asiat. Soc., Bengal, and ex c. British Museum [sent as "L. mauritiana" !]

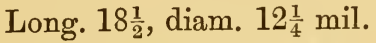

1 Port Jackson; coll. J. Caldwell, Esq.

\section{Littorina pulchra.}

Littorina pulchra, Swainson, Sowerby's Gen. Shells, 1820-4, fig. 2, sect. Littorinopsis.

3 Panama; coll. Prof. E. C. Stearns.

\section{Littorina nebulosa.}

Phasianella nebulosa, Lamarck, Hist. Vol. VII, 1822, S. Domingo; fide Mörch, Phil. Abb. V, figs. 19 and 20 as L. columellaris, also L. tigrina, Orb., Cuba, pl.15, figs. 9-10; sect. Littorinopsis.

4. Jamaica; coll. Prof. W. Newcomb [as "L. columellaris"].

5 Aspinwall; coll. Prof. E. C. Stearns [as "L. columellaris"].

\section{Littorina columellaris.}

Littorina columellaris, d'Orbigny, Voy. Amer. Mérid. 1840, Pernambuco; fide Mörch, long. 13, diam.9 mil., also from Martinique and? var. of L. flava, Brod. 1832 ; sect. Littorinopsis.

2 Jamaica; ex c. Prof. W. Newcomb.

Long. 13, diam. 9 mil.; agrees exactly with Mörch's descrip. tion, Malak. Blät. XXIII, page 135, and seems to me distinct from preceding. It resembles Reeve's fig. 96, L. sayi, Phil., from Florida. 


\section{Littorina penitaria.}

Littorina penitaria, Wood [ubi ?] ; ? sect. Melaraphe.

12 San Juan Fernandez; coll. Prof. E. C. Stearus [as "L. penitaria, Wood"].

Neither in Reeve, Philippi, nor in the Index Test.!

\section{Littorina irrorata.}

Turbo irroratus, Say, Journ. Philad. D, 182.2, June, p. 239 ;=Phasianella sulcata, Lamarck, 1822, August, p. 54; sect. Littorinopsis.

16 Florida and Hog Island [Virginia]; coll. Rev. E. R. Beadle, Prof. E. C. Stearns, and J. Lea, Esq. [As. Soc. Beng.].

\section{Littorina mauritiana.}

Phasianella mauritiana, Lamarck, Hist., 1822, Mauritius $=L$. lavis, Phil. [not of Reeve], P. Zool. S. 1845, Hab.? ; fide v. Martens, from Hongkong and Pt. Jackson; sect. Melaraphe.

6 Mauritius; coll. G. Nevill and J. Caldwell, Esqrs.

Very closely allied to $L$. obesa, but smoother and of different coloration. They may be only varietal forms? These specimens are most admirably and exactly represented by Philippi, III, Pl. VI, fig. 6, as $L$. loevis, Locality? The $L$. loevis of Reeve, I need scarcely say, is a totally different Australian form. Prof. v. Martens would rather consider $L$. lovis, Phil., as a form of L. glabrata.

\section{Littorina obesa.}

Littorina obesa, Sowerby, Gen. Shells, II, 1820-4, fig. 6, South Sea Islands; vars. $=L$. glabrata and $L$. debilis, Philippi; sect. Melaraphe.

5 South Sea Islands; ex c. Dr. J. C. Cox.

5 Maré, Loyalty Islands; ex c. F. Layard, Esq.

\section{var. glabrata.}

Littorina glabrata, Philippi, P. Zool. S. 1845, and Abb. pl. PII, fig. 5, Peru [!] ; subvar.=L. debilis, Philippi, l. c., without Loc.; fide Mart. as "L. glabrata," from Seychelles, Mascarene Isl., Natal and Singapore.

Scarcely specifically separable from $L$. muritiana, as already pointed out by Philippi [under L. lcevis]. Krauss records it 
as L. glabrata from Natal, and says "scarcely separable from $L$. obesa." It is also close to $L$. undulata, Gray, but differs in shape, \&c.

40 Seychelles, Mauritius, Ceylon, and Madras; coll. G. Nevill, Esq.

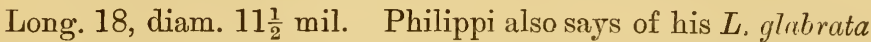
that it only differs by the coloration from $L$. obesa and $L$. loevis.

\section{Iittorina cincta.}

Littorina cincta, Quoy, Voy. Astrol. 1832, N. Zealand [not of Gould] ; = L. luctuosu, F. W. Hutton, J. de C., 1877 [not of Reeve].

10 Chatham Islands; ex c. Dr. F. Stoliczka.

\section{Littorina antipodum.}

Littorina antipodum, Philippi, Abb. II, 1847, N. Zealand [bene] $:=\boldsymbol{L}$. luctuosa, Reeve, 1865, N. Zealand $;=L$. diemenensis, Hutton, l. c. [not of Quoy].

15 New Zealand; ex c. Asiat. Soc. Bengal and Dr. F. Stoliczka $\left[13,7 \frac{1}{2}\right]$.

Profs. v. Martens and Hutton do not appear to have noticed this form, so admirably represented by Philippi. In coloration it much resembles $L$. diemenensis and has been confounded with it by some authors? The shape is quite different, the spire being more elongately produced, \&c. In some respects it also resembles Reeve's frgure of $L$. lavis. It is smoother and more regularly pyramidical, \&c., than the preceding.

\section{Littorina undulata.}

Littorina undulata, Gray, Zool. Beechey's Doy. 1839, Hab.?, "smooth"; from New Caledonia, fide Fischer, and from Aru, fide T.-Canefri.

100 Ceylon, Nicobars, Andamans, Penang, Arakan, Laccadives, and Madras; coll. R. C. Temple, F. Stoliczka, and G. Nevill, Esqrs.

1 Annesley Bay; coll. W. T. Blanford, Esq.

10 N. Caledonia ; ex c. P. Bouvier, Esq.

subvar. contracta.

20 Andamans; coll. G. Nevill, Esq.

The subangulation of two or three of these specimens is almost obsolete and may connect the form with $L$. columna, Philippi, Abb., Pl. VI, fig. 15, Loc.? 
var. sulcatula [? L. sulcatula, n. sp.].

Reeve, fig. $67 \mathrm{~A}$, as L. undulata, var.

5 Ascension Island, Pacific; coll. H. Pease, Esq.

\section{Littorina newcombi.}

Littorina newcombi, Reeve, Iconica, fig. 28, 1857, Sandwich Islands; var. =L. ambigua, Reeve, fig. 64 [not of Philippi=L. intermedia].

6 Sandwich Islands; coll. H. Pease, Esq.

Fischer records the species from New Caledonia, but I should imagine this form would rather be a variety of $L$. intermedia, if indeed the two species can be kept apart? Von Martens in the Donum Bism. considered $L$. ambigua as a synonym of $L$. pintado; this can hardly be the case.

var.

Recve, fig. 64, as L. ambigua, Philippi, Sandwich Islands.

10 Sandwich Isl. ; coll. Prof. W. Newcomb.

\section{Littorina varia.}

Littorina varia, Sowerby, Genera of Shells, 1823, fig. 4, Panama.

10 Panama ; coll. Profs. W. Newcomb and E. C. Stearns.

\section{Iittorina fasciata.}

Littorina fasciata, Gray, Zool., Beechey's Voy., 1839, Peru.

10 Panama; coll. Profs. W. Newcomb and E. C. Stearus.

\section{Littorina angulifera.}

Phasianella angulifera, Lamarck, Hist. VII, 1922, Antilles;-L. scabra, Orb. [not of Lin.]; sect. Littorinopsis.

10 Florida ; coll. Prof. E. C. Stearns. 4 Panama; coll. Prof. W. Newcomb.

1 Guadaloupe; ex c. Prof. W. Newcomb. 
There is no figure, in either Philippi or Reeve, that at all well represents any of these specimens.

\section{Littorina scabra.}

Helix scabra, Linnceus, Syst. Nat. X, 1756, Hab.?; from Madagascar, file IIts.; from Aru, fide T.-Canefri.

2 Mauritius ; coll. G. Nevill, Esq. $\left[26 \frac{1}{2}, 15 \frac{1}{4}\right]$.

A beautiful form with uninterrupted "flames," resembling in this respect Philippi's figs. 14 and 15 [L. angulifera, var].

1 Bombay; coll. Rev. S. B. Fairbank.

A most remarkable, turriculate specimen, perhaps abnormal! Long. 29, diam. $16 \frac{3}{4}$ mil.; anfr. 7 .

6 New Caledonia; ex c. P. Bouvier, Esq.

subvar. fiammulata.

Philippi, Abb. II, 1847, pl. V, fig.3, Philippines and Singapore; Reeve, figs. $21 \mathrm{~B}$. and $C$.

50 Seychelles $\left[36 \frac{1}{2}, 22 \frac{1}{2}\right]$, Andamans $[24,18]$, Mauritius, Maldives, Singapore, Ceylon, Nicobars ; coll. F. Stoliczka, J. Wood-Mason, and G. Nevill, Esqrs.

A small form, with distended, slightly patulous aperture, I take to be the male?

6 Kingsmill Island [Pacific 0.]; coll. H. Pease, Esq.

subvar. punctata.

Philippi, l.c. fig. 5, Philippines and Singapore; ?=var. articulata, Philippi, l. c. fig. 4, Phulippines, \&c.

12 Bombay, Sejchelles, Ceylon, Andamans, and Mauritius; coll. G. Nevill, Esq.

1 China; ex c. Prof. W. Newcomb.

3 Manila; coll. R. Hungerford, Esq. 
subvar. lutea.

Philippi, l.c., fig.11 [not 6], Plilippines, and Canton;=var. suturalis, Phi. lippi, l. c.fig. 10 [not 7]; =var. rubra, Philippi, l. c. for Chemnitz, fig. 2075, Canton and Philippines.

3 Mauritius; coll. G. Nevill, Esq.

1 Katchall, Nicobars ; coll. F. A. de Roepstorff, Esq.

All young, indifferent specimens; they may not be $L$. scabra at all.

subvar. tenuis, nov.

12 Arakan; coll. H. F. Blanford, Esq.

Remarkably thin, spire rather short, last whorl ventricose, with keel not much developed, aperture unusually large; occasionally has some varices behind the peristome.

Long. 31, diam. 22 mil.

\section{Iittorina intermedia.}

Littorina intermedia, Philippi, Proc. Zool. Soc., 1845, and Abb., pl. $T$, figs. 6-9 [not 8 to 11]; Red Sea, Natal, Australia, Philippines, Tahiti, Elizabeth Island, and Mergui; Reeve, fig. 101 ["type"]; Madagascar, fide Martens; Suez, fide McAndrew.

6 Navigators' Islands and Kingsmill Group; coll. H. Pease, Esq., and Dr. C. E. Tonnerre.

100 Andamans, Nicobars and Maldives $\left[26 \frac{3}{4}, 17 \frac{1}{2} !\right]$, Ceylon, Kathiawar, Singapore, Arakan, and Penang; coll. F. Stoliczka, J. Wood-Mason, G. Nevill, and F. Fedden, Esqr's., and ex c. Asiatic Soc. Bengal.

2 Cape; ex c. G. Nevill, Esq. [in bad condition].

4 Annesley Bay; coll. W. T. Blanford, Esq.

\section{var. strigata.}

Littorina intermedia, var. strigata, Philippi, Abb. pl. $V$, figs. 7-9, Mergui [not L. strigata, Lischke].

12 Nagasaki ; coll. R. Hungerford, Esq. [13 $\left.\frac{1}{2}, 8 \frac{3}{4}\right]$.

Only differs from the Indian Ocean form by the columella being throughout stained of a dull purple colour, instead of being white with a brown edging. 
100 Port Canning, Penang, Singapore, Rangoon, Amherst and Akyab; coll. H. F. Blanford, J. Dodgson, F. Stoliczka, and G. Nevill, kisqrs.

Some marked male and some female by Dr. Stoliczka. I can see no difference!

15 Bombay ; coll. Rev. Fairbank, and W. T. Blanford, Esq.

6 Mauritius; coll. G. Nevill, Esq.

These last a slightly different form.

var. sinensis.

Littorina sinensis, Philippi, Abb. II, 1847, pl. VI, fig. 23, China; subvar.=

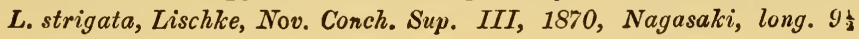
diam. 6 mil.

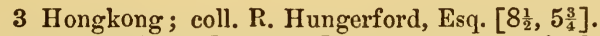

10 Anmungkang [Formosa]; coll. R. Hungerford, Esq.

The L. [Melaraphe] blanfordi, Dunker, Malac. Blät., 1871, Rockhampton, long. 12, diam. 8 mil., is apparently a close ally?

\section{Iittorina arboricola.}

Littorina arboricola, Reeve, Icon., fig. 27, 1857, Singapore.

20 Andamans; coll. Colonel E. S. Berkeley, and F. A. de Roepstorff, Esq.

A smaller form than the type, somewhat approaching some varieties of Littorina conica, Phil., and equally variably and prettily coloured; the spiral sculpture rather less developed.

5 Singapore; coll. Dr. F. Stoliczka.

I am very loath to adopt this species as distinct; the differences from $L$. scabra are evident at a glance; $L$. pallescens, Phil. and L. philippiana, Reeve, are the nearest I know; the more convex whorls, thinner substance, absence of keel at the periphery, and the rather peculiarly "excavately appressed" columella seem, however, characteristic! In size and coloration all five of the above resemble the type.

Long. $18 \frac{1}{4}$, diam. $11 \frac{3}{4}$ mil. 
var. philippiana.

Littorina philippiana, Reeve, Iconica, fig. 22, Philippines; also fig. 21 A ("L. scabra.")

4 Manila ; coll. R. Hungerford, Esq.

Long. 19, diam. $11 \mathrm{mil}$.

Of uniform yellow coloration, resembling Reeve, fig. $21 \mathrm{~A}$, but sculpture more distinct.

2 Port Curtis; coll. Dr. J. C. Cox.

A rather larger form than the preceding, long. 24, diam. 15, of equally thin texture. Fischer records L. philippiana from New Caledonia.

\section{Littorina pallescens.}

Littorina pallescens, Philippi, P. Zool. S., 1845, Philippines; ? var. $=L$. sieboldii, Philippi, 1845, from Japan; var. $=$ L. scabra, Reeve (pars) and L. intermedia, Phil. (pars).

5 Kingsmill Group; coll. H. Pease, Esq.

One only of these five has the four raised keels, the others closely resemble the Singapore specimens, also Reeve's fig. $21 \mathrm{~A}$, and Philippi, pl. V, figs. 10-11.

5 Navigators' Islands [Pacific]; ex c. Asiatic Soc., Bengal.

P var. erronea.

Philippi, pl. D, figs. 10-11, as L. intermedia, var.; Reeve, fig. $21 \mathrm{~A}$ as $L$. scabra, var.

4. Singapore; coll. Dr. F. Stoliczka $\left[28 \frac{1}{2}, 16\right]$.

Mr. H. F. Blanford also possesses a fine series of this form; the sculpture is variable, sometimes as in the figures quoted, but oftener more developed, much as in Reeve's fig. 22.

\section{Littorina filosa.}

Littorina filosa, Sowerby, Genera of Shells, 1820-4, "S. America"; var.= I. cingulata, Philippi, P. Zool. S., 1845, N. Australia; subv.?-L. sulculosa, Philippi,l.c., N. Australia.

1 Tahiti; ex c. Mus. Godeffroy.

The exact image of Sowerby's type figure! 
var. subcingulata, nov.

Distinguished from L. scabra and all its allies by the rounded thin columella which is not in the least thickened or appressed; the coloration is much as in L. scabra var. punctata, Phil.; the spiral ribbing more numerous and less raised than in typical $L$. filosa.

Long. 17, diam. 10 mil.

5 Port Jacbson; ex c. Dr. J. C. Cox and British Museum.

Sent by both as L. scabra.

\section{Littorina conica.}

Littorina conica, Philippi, P. Zool. S., 1845, Mergui (Burma) and Java, and Abb. $\nu$ l.VI, fig. 1-2; fide Reeve from Japan [? erron.].

2 Port Canning; coll. J. Wood-Mason, Esq. $\left(17 \frac{3}{4}, 11\right)$.

Agree exactly with original figures; Mr. H. F. Blanford also possesses four, probably typical (?) specimens from Tenasserim [Mergui ?].

var. delicatula, nov.

30 Port Canning and False Point (Bengal) ; coll. Colonel G. B. Nain . waring, G. Nevill, Esq., and Dr. F. Stoliczka.

Perhaps the most beautiful marine shell, when living, that I have ever seen. The colours fade terribly after death; it is most difficult to get perfect specimens and it appears to be remarkably local. The variety can be distinguished by its extreme fragility, by the much less developed peripheral keel, less obsolete sculpture, and especially by the more rounded columellar margin at base; it varies wonderfully in colour, from a beautiful rose to the typical straw colour, oftentimes marbled or striped. I had separated the above from the following specimens as a distinct sub-variety, when I found two tubes in Dr. Stoliczka's collection, marked " $f$ and $q$ "; that marked $q$ contained six of the preceding and one of the following specimens [the last probably inserted erroneously ?]; the other tube marked ơ contained eight, all of the following form. 
Here perhaps is an explanation of some of the differences in allied forms? Can, for instance, my Singapore specimens catalogued as L. pallescens var. erronea, and those as L. arboricola var. philippiana, be the different sexes of one species?

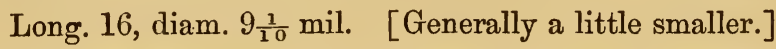

The differences from the typical form are probably due to my var. delicatula living in slightly brackish water? Reeve's L. tenuis from the Philippines seems to be an allied form?

20 Port Canning and False Point; coll. Colonel G. B. Mainwaring, Dr. F. Stoliczka, J. Wood-Mason and G. Nevill, Esqrs.

Of equally fragile texture, but easily distinguished from the female form by the less produced spire, even less developed keel at periphery, and especially by the conspicuously distended aperture and rounded columellar margin at base; several specimens have one or two varices behind the peristome, which is occasionally "subduplex." The coloration is, if possible,

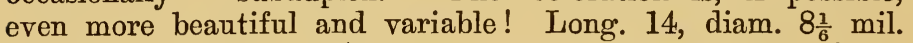
Gibbons, Q. J. Con. 1878, p. 339, states that specimens of Lit. intermedia and angulifera become less solid as the water in which they live is less salt; I am not sure that this applies to the form under consideration as immediately associated with my var. delicatula, may be found normally solid Lit. intermedia and the very thick-shelled Lit. melanostoma.

\section{var. subintermedia, nov.}

10 Port Canning and False Point; coll. Colonel G. B. Mainwaring.

Long. $15 \frac{1}{2}$, diam. 9 mil.

Most remarkable and interesting specimens, combining many of the characters of $L$. intermedia, Phil., with those of $L$. conica. Can they be a hybrid race?

The above are what I take to be the $\rho$ form.

8 Port Canning; coll. Colonel G. B. Mainwaring.

These are doubtless males.

\section{Littorina albicans.}

Littoriua albicans, Metcalfe, P. Zool. S., 1852, Borneo.

3 Loc. ?; ex c. Dr. F. Stoliczka. 
Agree well with Reeve's fig. 44, only the characteristic subacute spiral striation is much more distinct.

\section{Littorina melanostoma.}

Littorina melanostoma, Gray, Beechey's Voy. 1839, p. 140, Indian Ocean; Seychelles, fide Mts.; Java, Mergui, and Ceylon, fide Phil.; Singapore and Borneo, fide Reeve.

10 Port Canning; coll. Dr. F. Stolizka (marked " $f$ ").

5 Port Canning; coll. Dr. F. Stoliczka (matked " $q$ ").

I can trace no difference whatever, except it be that the female form seems a little larger.

20 Port Canning, Akyab, False Point, Masulipatam, and Amherst; coll. J. Wood-Mason and G. Nevill, Esqr's., and Colonel G. B. Mainwaring.

\section{var. articulata.}

5 Hongkong; coll. R. Hungerford, Esq.

Long. 12, diam. $6 \frac{1}{2}$ mil.

A small form, with articulate brown spots, instead of more or less undulating longitudinal stripes, sent me as only found in one spot, and rare there.

\section{Littorina carinifera.}

Phasianella carinifera, Menke, Syn. II, 1830, Hab. ?; fide Philippi, from Java, China, Philippines and $=L$, perdix, King, 1831; fide Reeve, from Borneo, \&c.

24 Andamans and Nicobars; coll. F. Stoliczka and J. Wood-Mason, Esqrs. 12 Bombay and Arakan; coll. Rev. S. B. Fairbank and H. F. Blanford, Esq.

subvar. pyramidalis.

4. Bombay and Java (?); coll. Rev. S. B. Fairbank and ex c. Madras Museum.

var. lævior.

Long. 16, diam. $10 \frac{1}{8} \mathrm{mil}$.

12 Penang and Singapore; coll. Dr. F. Stoliczka. 


\section{Littorina ventricosa.}

Littorina ventricosa, Philippi, Abb. III, pl. VI, fig. 19, 1851, Penang; var. $=L$. subgranosa, Frauenfeld, [pars]; var. ?=L. granularis, Gray .

1 Bombay $\left[10 \frac{1}{4}, 7 \frac{3}{4}\right]$; coll. W. T. Blanford, Esq.

4 Hongkong; coll. R. Hungerford, Esq.

50 Arakan, Andamans and Penang; coll. R. Hungerford, F. Stoliczka, R. C. Temple, and G. Nevill, Ésqrs.

Philippi's figure is unmistakeable, whereas Reeve's, fig. 93, is very indifferent. The sculpture is very variable, occasionally unusually developed. Mr. Smith, P. Zool., S. 1878, appears to consider this Andaman form as $L$. ventricosa and compares it with his $L$. melanacme and $L$. novae-zelandice.

\section{var. subgranosa.}

Melaraphe subgranosa, von Frauenfeld, Novara-Reise, pl. I, fig. 10 [not A. B.] from Madras ; ?=L. granularis, Gray, Beechey's Voy., 1839, N. Australia and of Reeve, fig. 68.

6 Andamans; coll. Captain R. C. Temple.

7 Irawady Delta; coll. W. T. Blanford, Esq.

1 Galle Harbour; coll. Dr. J. Anderson.

Long. 9, dian. [vix] 8 mil. This form, exactly represented by Frauenfeld's fig. c., of coarser sculpture than the type, is undoubtedly only a var. of $L$. ventricosa ; if it prove to be Gray's species, as I expect it will, this latter name will have priority. The specimen, from the same province of Ceylon as the next form, $L$. nove-zelandice, has induced me to believe the latter a distinct species? I can perceive, however, no difference except that of sculpture, and the shorter, more ventricose form of $L$. ventricosa and its var. subgranularis, both characters very variable in this species.

\section{Littorina erronea, n. sp.}

Littorina novæ-zelandiæ, Reeve, 1857, N. Zealand.

10 Balapiti, Ceylon; coll. G. Nevill, Esq.

Long. 13, diam. $10 \frac{1}{2}$ mil.

I am convinced this species was never found in New Zealand. Prof. F. W. Hutton in 1878 records that he had never seen specimens. As to the localities of Reeve and von Frauenfeld 
[Auckland], they are utterly without value in my eyes; the latter's specimens probably came from Madras? The above recorded specimens are a constantly much smoother form than L. ventricosa, with even less marked coloration and distinctly more produced spire in all cases, though this last is a variable character in both species. E. A. Smith describes a closely allied, but more compressed form, from the Solomon Islands, as $L$. melanacme, P. Linn. Soc. XII, 1876, pl. XXX, fig. 21 ; this may be the $L$. novoe-zelandice of Fischer from New Caledonia?

\section{Littorina reticulata.}

Littorina reticulata, Anton, Verz. Conch., p. 53, Hab.? and Philippi, Abb. pl. IV, fig. 12; ?=L. granocostata, Reeve, 185\%, fig. 79, Brisbane; Mascarene Islands, fide Martens.

7 Praslin Islands, Seychelles; coll. (x. Nevill, Esq.

I took this distinct little species alive at one spot on Praslin, and never saw it elsewhere from these seas, unless the Andaman specimens, qunted below, should prove to belong to it. My specimens agree exactly with Reeve's figure and description.

Long. 7, diam. 5 mil.

\section{Littorina millegrana.}

Littorina millegrana, Philippi, Abb. III, 1850, pl. VII, fig. 15, Red Sea ; Suez, fide McAndrew.

5 Meshek [Persian Gulf]; coll. W. T. Blanford, Esq.

Not in a satisfactory condition.

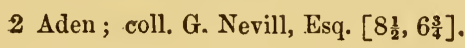

var. cinerea.

Littorina cinerea, Pease, Amer. Journ. Conch. IV, 1870, pl. VIII, fig. 14, Marquesas; alt. 7, diam. 5 !ٕ mil.

10 Balapiti, Ceylon; coll. G. Nevill, Esq.

These agree exactly with Pease's above-quoted figure, the spire is relatively higher than in Philippi's figure; the species is a connecting link with the Sub-Genus Tectarius; $\mathrm{H}$. and A. Adams indeed considered it a species of that Genus. 


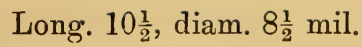

2 Mauritius ; coll. G. Nevill, Esq. $\left[7 \frac{3}{4}, 5\right]$.

3 Tahiti; ex Mus. Godeffroy.

10 Andamans; coll. J. Wood-Mason, Esq.

Unfortunately these last are not quite adult; apparently the sculpture of this species at the Andamans is most variable. Some have the characteristic, longitudinal, undulating furrows of $L$. granocostata.

\section{Littorina miliaris.}

Littorina miliaris, Quoy, 1832, pl. 33, figs. 16-19, "Ile de l'Ascension"

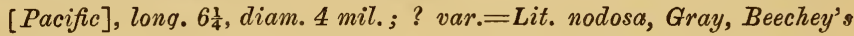
Voy. 1839, N. coast of Australia [not Philippi,pl. III, figs. 6-7?]; ?=Lit. exigua, Dunker, Fauna Japonica, 1861, pl. II, fig. 3 ; ? worn var. $=L$. leucosticta, Philippi, pl. III, fig. 11, Locality? ; ? var.= Lit. subnodosa, Philippi, Red Sea.

2 Madagascar; coll. J. Caldwell, Esq.

3 Nagasaki; coll. R. Hungerford, Esq.

12 Hongkong; coll. R. Hungerford, Esq.

Long. 10, diam. 8 mil. Very variable as regards sculpture, sometimes only spirally striate, occasionally more or less distinctly granulose; it can always be easily distinguished from L. ventricosa, which lives with it, by being always angulate at the base of the aperture, instead of rounded. It is a larger, coarser variety than the Ceylon form, and may be specifically distinct; it can be easily distinguished by the more regular spiral sculpture and its sombre coloration, wanting the characteristic black and white "filleted" bands [as seen also in Philippi's fig. of $L$. leucosticta]; it is as much like Philippi's figure of $L$. subnodosa as that he gives of $L$. nodosa, Gray. Reeve's fig. 13 of $L$. nodosa fairly represents some of the Hongkong specimens.

var. fijiensis.

Littorina fijiensis, Reeve, Icon. 1857 ; emend. Martens in Donum Bism. as $\mathbf{L}$. vitiensis.

2 Tahiti ; coll. H. Pease, Esq. [sent as "L. fijiensis "].

Undoubtedly, I think, not specifically separable from the preceding. It can scarcely be the $L$. vitensis of Dunker, Malac. Blät. 1871, Fiji Isl., long. 10, diam. 7 mil. ? 
var. subgranosa.

Littorina subgranosa, von Frauenfeld [pars], Novara Reise, pl. I, fig. $10 \mathrm{~A}$ and $B[$ not $C]$, Madras.

8 Galle; ex c. Asiatic Soc., Bengal.

Agree with Philippi's Pl. III, fig. 11.

40 Ceylon; coll. G. Nevill, Esq.

The largest specimen measures, long. 11, diam. $7 \frac{1}{2}$ mil.

20 Akyab and Irawady Delta; coll. W. T. Blanford, Esq., and Dr. F. Stoliczka.

These agree well with the above-quoted figs. A and B. of $\nabla$. Frauenfeld.

2 Annesley Bay; coll. W. T. Blanford, Esq.

These last are a small form; they can scarcely be the $L$. subnodosa of Philippi, McAndrew, \&c., said to be from the Red Sea?

Sub-Genus TECTUS, Montfort.

Con. Syst. II, 1810, type Turbo pagodus, Lin.;=Pagodus, Gray, Beechey's Voy. 1839, type T. pagodus, Lin.;=Pagodella, Swainson, 1840, type P. major, Martyn, fig.1541.2 ; fide Mörch, Malac. Blät. 1876=Echinella, Swainson.

There is a Tectus montrouzieri, Fischer, 1878, from N. Caledonia, the generic classification of which, however, seems to me very doubtful; it possesses a distinct tooth on the columellar margin. Issel has figured a Tectaria armata from the Gulf of Suez.

\section{Littorina (Tectus) pagodus.}

Turbo pagodus, Linnceus, Syst. Nat. X, 1758, p. 762, O. asiatico; Zanzibar, fide Cuming [?], N. Ireland, fide Quoy, Amboinu, fide Rumphius.

3 Torres Straits; ex c. G. Nevill, Esq., and Dr. Cox.

\section{Littorina (Tectus) muricata.}

Turbo muricatus, Linnaus, Syst. Nat. X, 1758, Jamaica.

12 Florida Keys and Gulf of Mexico; coll. Prof. E. C. Stearns and J. Lea, Esq. [As. Soc., Beng.]

8 S. Domingo; coll. Prof. W. Newcomb. 


\section{Isittorina (Tectus) pyramidalis.}

Littorina pyramidalis, Quoy, Iroy. Astrol., 1832, pl. XXXIII, figs. 12, 15, N. Zealand.

4 Australia ; ex c. Asiatic Soc., Bengal (coll. Strange).

6 Port Jackson; cull. Dr. J. C. Cox.

var. vilis.

Littorina vilis, Menke, Phil. Abb., pl. II, fig. 21, 1847, Hab. ?; Reeve, fig. 12, from $N$. Zealand.

Unfortunately one or two of Dr. Stoliczka's cabinet-trays had got mixed before they came under my charge.

5 Nukahiva [?] ; ex c. Dr. F. Stoliczka $[13,9]$.

58. Littorina (Tectus) tuberculata.

Littorina tuberculata, Menke, Syn. Ed. I, 1826; =L. trochiformis, "Dillwyn", Phil. Abb., pl. II, fig. 12.

2 Guadaloupe; coll. Prof. W. Newcomb.

\section{Littorina (Tectus) dilatata.}

Littorina dilatata, d'Orbigny, Moll. Cuba, 1841; fide Mörch,? var. of $L$. tuberculata.

2 Cuba; coll. Prof. W. Newcomb.

This seems to me only a variety of the preceding.

\section{Littorina (Tectus) armata.}

Tectaria armata, Issel, Malac. Mar Rosso, 1869, Suez.

4 Gwadar and Henjam (Persian Gulf); coll. W. T. Blanford, Esq.

\section{Littorina (Tectus) trochoides.}

Littorina trochoides, Gray, Beechey's Toy., 1839 (N. Australia ?); L. malaccana, Phil. Abb., pl. VI, fig. 17, 1851, Penang; ?=L. natalensis, Krauss, Philippi, pl. IlI, fig. 4, Natal.

12 Hongkong; coll. R. Hungerford, Esq.

100 Andamans, Aden, Persian Gulf, Annesley Bay, Arakan, Bombay, Madras, Ceylon, and Bombay; coll. W. T. Blanford, F. Stoliczka, and G. Nevill, Esqrs.

4 Marquesas ; coll. H. Pease, Esq. (as "L. trochoides"). 
These last agree well with Indian Ocean specimens; they measure-long. $12 \frac{1}{2}$, diam. 8 mil.; the sculpture oftentimes becomes subobsolete, and the length of the spire varies in specimens from the same locality.

\section{Sub-Genus ECHINELLA, Swainson.}

Treat. Malac., 1840, type E. coronaria, Lamarck.

\section{Littorina (Echinella) cumingi.}

Trochus cumingi, Philippi, P. Zool. S., 1845, Guimaras (erron.?).

3 Roratonga; coll. H. Pease, Esq.

\section{Littorina (Echinella) coronaria [?]}

Monodonta coronaria, Lamarck, Hist., 1822, Hab.?; Lord Hood's Island, fide Cuming; Hervey Island, in Cat. Mus. Godeffroy.

5 Roratonga; coll. H. Pease, Esq., and Asiatic Soc., Bengal.

These all agree best with Philippi's figures 2 and 6 of Pl. II, said to be L. bullata, Martyn, from N. Zealand [=L. papillosa, Lam.]; I preserve as above, however, the name under which they were sent me by Mr. Pease ; from von Marten's remarks in the Donum Bism., the distinctiveness of the two forms appears to be very doubtful. Philippi gives as localities for $L$. bullata Zanzibar and Swan Point [Australia].

Genus LimNotrochUs, E. A. Smith.

Ann. Mag. 1880, type L. kirki, Smith.

E. A. Smith describes the operculum of Limn. thomsoni as" horny, paucispiral and littorinoid."

\section{Limnotrochus kirkii.}

Limnotrochus kirkii, E. A. Smith, Ann. Mag., 1880, Lake Tanganyika.

1 Lake Tanganyika [typical]; ex Mr. Damon.

Genus PLESIOTROCHUS, Fischer.

J. de C., 187S, type P. souverbianus, Fischer [Loyalty I.]. 

\&c.

Very probably belongs to the Litiopina, near Alaba, Diula,

\section{Plesiotrochus, n. sp.}

An interesting form of this very distinct new Genus which I have for several years intended to describe. The whorls are characteristically concave.

10 Mauritius; coll. G. Nevill, Esq.

1 Andamans; coll. Dr. F. Stoliczka.

2. Plesiotrochus [?] n. sp.

2 Persian Gulf ; coll. W. T. Blanford, Esq.

Sub-family FOSSARIN $A$, Stoliczka.

Genus RISELLA, Gray.

Syn. Brit. Mus. 1840, type Trochus melanostoma, Gm. ; = Bembicium, Philippi, Zeits. Malac. III, 1845.

\section{Risella plana.}

Trochus plana, Quoy, Voy. Astrol. 1832, "Port Western," Bass's Strait; =Littorina australis, Gray, Beechey's Voy. 1839 ; var. $=$ R. grisea, Phil., Zeits. Mal. 1851, Hab.?

7 Australia ; ex c. Asiat. Soc., Bengal [coll. Strange].

4 South Australia; ex c. British Museum and Miss Pelly.

Agree with figure, in Conch. Cab. II, of $R$. grisea, Phil.

var.

1 Port Mackay; ex Mus. Godeffroy [as "var. B., Crosse"].

30 Port Jackson; coll. Dr. J. C. Cox and G. Nevill, Esq.

\section{Risella imbricata.}

Littorina imbricata, Gray, Beechey's Voy., 1839 [sine Hab.]; = Risella kielmannseggi, Frauenfeld, 1866, N. Zealund.

1 Australia; ex c. G. Nevill, Esq. 


\section{Risella nana.}

Trochus nana, Lamarck, Hist. 1822, Australia $=$ Bembicium pictum, Phil. Zeits. Mal., 1846, Van Dieman's Lasd, fide Phil. = R. nana Quoy [not Lam.].

4 Tasmania ; ex c. G. Nevill, Esq.

\section{Risella lutea.}

Trochus luteus, Quoy, Voy. Astrol.1832, "Port du Roi-George"; =Trochus cicatrosus, Jonas, Phil. Abb. 1843, Port Western.

The Rev. Tenison-Woods considers this species as a sexual variety of $R$. plana.

30 Pt. Jackson and Moreton Bay; coll. G. Nevill, Esq.

\section{Sub-Genus PEASIELLA, nov.}

Type, Trochus tantillus, Gould.

E. A. Smith in the Journal, Linnæan Soc., XII, 1876, p. 552, describes the operculum of $R$. tantilla as "truly appertaining", to the Family Trochida, being concentric and multispiral, but that for other reasons he considers it should be located with the Genus Risella.

A species from the Loyalty Islands has been described by Montrouzier, J. de C., 1879, as "Echinella gaidei, long. $3 \frac{1}{2}$, diam. $4 \frac{1}{2}$ mil."; apparently this must be a close ally of the Andaman form. I have named this new group in honour of the late Harper Pease, Esq. ; it is easily distirguished by the perforate or umbilicate base, \&c.

\section{Risella [Peasiella] tantillus.}

Trochus tantillus, Gould, P. Bost., S. III, 1849, Sandwich Islards.

20 Sandwich Islands; coll. H. Pease, Esq., Dr. H. Dohrn, and Prof. W. Newcomb.

var. parvula.

Risella parvula, Dunker, Cat. Mus. Godef. IV, 1869, Samoa.

1 Samoa; ex Mus. Godeffroy. 
Judging from this singie specimen, a very distinct small form. Sub-tuberculate above, striate [instead of sulcate] below. Perhaps the form described by Smith, l. c., as found by Brazier at the Marshall Islands.

var. [? dist. sp.].

Long. 3, diam. $2 \frac{3}{4}$ mil. Differs from the Arakan form of $R$. isseli by the still narrower perforation, more convex base, with more regular and more numerous spiral sulcations.

8 Cherboniane Reef; coll. Dr. J, Armstrong.

2 Mauritius; ex c. Dr. F. Stolizcka [in bad condition; identification doubtful].

var. subinfracostata [? dist. sp.].

Probably distinct; the specimens have their opercula, but are perhaps not quite adult? Descr. de l'Egypte, Pl. V, fig. 40, gives a good idea of them. It is perhaps rather a var. of that species, $R$. infracostata, Issel?

3 Nicobar Islands; coll. Dr. F. Stoliczka.

\section{Risella [Peasiella] conoidalis.}

Trochus conoidalis, Pease, Amer. Journ. Conch. III, 1867, Paumotus; alt. 5 , diam. 4 mil.

3 Paumotus [typical]; coll. H. Pease, Esq.

\section{Risella [Peasiella] infracostata.}

Risella infracostata, Issel, Malac. Mar Rosso, 1864, Suez and Desc. de l'Egypte, $p l . V$, fig. 40.

6 Massaua; coll. Prof. A. Issel.

Sent me as "Troch. sismonda, Iss.", probably a lapsus calami? I am inclined to believe it can only be separated from the following as a variety; my Aden specimens seem a fairly intermediate form.

\section{Risella [Peasiella] isseli.}

Risella isseli, Semper, Issel, Malac. Mar Rosso, 1861, Suez and Zunzibar; Descr. de l'Egypte, pl. $V$, fig. 35 . 
3 Muscat; coll. W. T. Blanford, Esq.

7 Red Sea ; ex c. Dr. H. Dohrn.

5 Roweiah and Aden; coll. J. B. Baxter and G. Nevill, Esqrs.

An intermediate form, as to sculpture, between the two figures in Descr. de l'Egypte.

\section{Risella [Peasiella] roepstorffiana, n. sp.}

20 Arakan; ex c. Dr. F. Stoliczka.

Uniform yellow coloration throughout; very small umbilicus; nearly smooth above, never subimbricate at the sutures, only very rarely slightly undulately "waved" at the periphery.

10 Andamans; coll. F. A. de Roepstorff, Esq.

Alt. $2 \frac{3}{4}$, diam. $3 \frac{3}{4}$ mil.

10 Mergui ; coll. Dr. J. Anderson.

5 Hongkong; coll. R. Hungerford, Esq.

"Lives on rocks, about high-water mark!" The narrow umbilicus resembles that of Red Sea specimens. Two of them possess a basal dark zone, as in $R$. templiana var. nigrofasciata. Not subimbricate.

\section{Risella [Peasiella] templiana, $\mathrm{n}$. sp.}

Exceedingly variable in coloration and sculpture; seems, however, to be constantly distinguished from the preceding forms by its considerably more open umbilicus, constantly stained of a rich chesnut; the columella seems to be more distinctly thickened and is of the same colour; the form I take for my type is evenly, spirally striate above, striation more or less obsolete on the apical two or three whorls; aperture of a uniform saffron yellow within; no subimbrication, or peculiar sculpture of any sort, at the suture; three or four deeply excavated spiral sulcations at base. Long. $3 \frac{1}{2}$, diam. 4 mil.

12 Andamans [type]; coll. Capt. R. C. Temple.

\section{var. nigrofasciata.}

The interior of the aperture shows two dark-brown bands, one quite close to the suture, the other quite at its base; a rather broad, dark-coloured band also round the base, just below 
the suture ; undulately "waved" or subimbricate at the suture, as in Descr. de l'Egypte, Pl. V, fig. 35, which indeed gives perhaps a better idea of this Andaman species than of the Red Sea form; intermediate forms will probably occur to connect the two?

4 Andamans; coll. Capt. R. C. Temple.

var. subimbricata.

With the uniform yellowish coloration of the typical form and the peculiar subimbricate sculpture at the sutures and periphery of the preceding variety.

6 Andamans; coll. Capt. R. C. Temple.

11. Risella [Peasiella], n. sp.

1 Muscat; coll. W. T. Blanford, Esq.

A very small form, with peculiar, perfectly flattened spire; below the acute periphery there is a deep and broad sulcation, the rest of the base appearing smooth, though under the lens a minute striation can be detected. The umbilical perforation is very small.

\section{Genus PHANETA, H. Adams.}

P. Zool. S. 18\%0, type P. everetti, H. Adams [Siniwar Rv., Borneo].

The correct classification of this Genus appears to be still very doubtful. Phaneta is not as yet represented in the Museum.

Genus FOSSAR, Gray.

Syn. Brit. Mus. 1840, type Helix ambigua, Lin. $;=$ Fossarus, Philippi, 1841 ; sect.=Clatkrella, Recluz, J. de C., XII, 1864, types Foss. costatus, Brocchi, and Foss. minutus, Michaud.

A. Adams figured a Fossar bicarinatus in the P. Z. S. for 1853 from the Red Sea ; he describes the operculum as " horny, oval and subspiral."

\section{Fossar ambiguus.}

Helix ambigua, Linnaus, Syst. Nat. 1758, Mediterranean ;=" le Fossar," Adanson, 1757, Senegal $;=$ Fossarus adansonii, Recluz [? not of Phil.] 
6 Algiers; coll. P. Joly, Esq.

1 Japan ; ex Mr. Damon ["coll. A. Adams"].

8 Ceylon; coll. G. Nevill, Esq.

4 Andamans [juv.]; coll. G. Nevill, Esq., and Captain R. C. Temple.

This is, I believe, one of the very few mollusks common to the European and Indian Seas; I cannot separate the two forms even as varieties! A. Adams records $F$. costatus, Brocchi, from the Mediterranean and China Seas; but $F$. ambiguus from Senegal only.

\section{Fossar tornatilis.}

Fossarus tornatilis, Gould, P. Bost. S. 1859, Hongkong, at 10 fths.-axis 5, diam. 4 mil. $=F$. stolizkanus, Nevill, J. As. Soc. Bengal, 1871, Ceylon, and Bombay, long. $4 \frac{3}{4}$, diam. $3 \frac{3}{4}$ mil. $;=F$. japonicus, $A$. Adams, Ann. Mag. 1861.

1 Japan ; ex Mr. Damon [" coll. A. Adams" as "F.japonicus"].

5 Bombay [typical F. stoliczkanus]; coll. Rev. S. B. Fairbank.

1 Gwadar [with operculum); coll. W. 'T. Blanford, Esq.

14. Hongkong; coll. R. Hungerford, Esq.

Fide A. Adams, P. Z. S. 1863, should be Nerita costata, Broc., a European species. Fischer, J. de C., 1864, separates the two forms. My brother also possesses the species from Ceylon.

\section{Fossar trochlearis.}

Fossar trochlearis, A. Adams, P. Zool. S. 1853, p. 187, Philippines; from Japan and Pegu,op. cit., 1863 ; fide McArthur, Suez.

1 Japan ; ex Mr. Damon [“coll. A. Adams"].

40 Andamans, Arakan, Muscat, Bombay and Ceylon; coll. F. Stoliczka, W. T. Blanford, J. Wood-Mason, and G. Nevill, Esqrs.

Lives on large oyster shells !

1 Hongkong; coll. R. Hungerford, Esq.

\section{4. [?] Fossar, n. sp.}

1 Arakan; coll. Dr. F. Stoliczka.

Very likely a species of Vanikoro; unfortunately it is in very bad condition. It bears some resemblance to $F$. reticulatus, but is covered with very peculiar tubercles, \&c., \&c. 


\section{Fossar, n. sp.}

1 Annesley Bay ; coll. W. T. Blanford, Esq.

Two prominent raised keels on the last whorl, with minute, very characteristic, spiral striation in the interstices, \&c., narrowly rimate only.

\section{Fossar, sp.}

1 Japan ; ex Mr. Damon (coll. A. Adams).

Labelled "F. bicarinatus, A. Adams."

This specimen, with three keels on the last whorl, seems to me not adult ; it is certainly not F. bicarinatus, A. Ad., P. Zool. S. 1853, Red Sea, which A. Adams does not record in his List of the Fossaridoe, P. Z. S. 1863, probably because the Red Sea species is a Separatista, and not a Fossar at all.

\section{7. [?] Fossar, n. sp.}

3 Mauritius; coll. G. Nevill, Esq.

In very bad condition; perhaps a sp. of Conradia? var. (? dist. sp.)

1 Annesley Bay ; coll. W. T. Blanford, Esq.

Also in very bad condition.

\section{Fossar fenestratus.}

Fossar fenestratus, A. Adams, P. Zool. S., 1863, Osima (Japan).

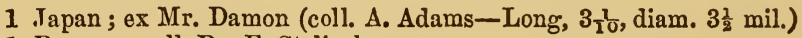

1 Penang; coll. Dr. F. Stoliczka.

Agrees exactly with the preceding specimen.

\section{Fossar garretti [?].}

Fossar garrettii, Pease, Amer. Journ., Conch. IV., 1868, for "Adeorbis costata", Garrctt, P. Calif. Acad. 1857, p. 103 (Polynesia, Pease).

1 Loc.?; ex Mr. Damon. 
Sent as $F$. sandwichensis. Possibly it may be $F$. multicostatus, Pease, P. Zool. S. 1860, from the Sandwich Islands? Long. $3 \frac{5}{8}$, diam. 4 mil.

10. Fossar, sp.

1 Locality? ; ex ¿. Colonel G. B. Mainwaring.

11. Fossar, n. sp.

1 Bombay; coll. Rev. S. B. Fairbank.

Long. $3 \frac{1}{4}$, diam. 2 mil. Like the preceding, but with raised spiral striation, instead of incised lines ; close to $F$. trochlearis, but umbilicus less open and defined, sculpture finer, \&c.

\section{Fossar mirabilis, n. sp.}

2 Arakan (type); coll. Dr. F. Stoliczka.

Long. $1 \frac{1}{2}$, diam. [vix.] $1 \frac{1}{2}$ mil.

A most remarkable form of rather thin substance, of two whorls only ; spire not raised at all, completely hidden in front by the last whorl, which in shape resembles that of $F$. tornatilis, it is girt with five acute and raised spiral keels, there being no transverse sculpture whatever; umbilical region well defined; peristome fimbriate.

\section{3. (?) Fossar imperforatus, n. sp.}

1 Penang (type) ; coll. Dr. F. Stoliczka.

Long. $3 \frac{3}{4}$, diam. $2 \frac{1}{2}$ mil.

Whorls $2 \frac{1}{2}$, spire scarcely raised, apex obtusely flattened; longitudinally, flexuously, distantly ribbed; ribs more or less obsolete, and towards the base, closely and coarsely spirally striated ; imperforate, of rather solid texture. Can scarcely be a sp. of Vanilioro.

\section{Fossar, sp?}

2 Andamans; coll. Captain R. C. Temple.

15. Fossar, sp ?

3 Hongkong; coll. R. Hungerford, Esq. 
A form allied to, but distinct from, $F$. fenestratus, A. Adams.

$$
\text { Sub-genus COUTHOUYIA, A. Adams. }
$$

Ann. Mag., 1860, type C. decussata, A. Adams.

A fossil species of Couthouyia is the Lacuna disjuncta, Rainc. and Munier, 1863.

\section{Fossar [Couthouyia] reticulatus.}

Fossar reticulatus, A. Adams, P. Zool. 1853, p. 186, Singapore, p. XX, fig. 13; from Japan, op. cit. $1863 ;=F$. insignis, G. \& H. Nevill, J. As. Soc.

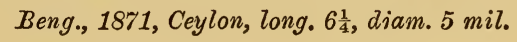

The two nuclear whorls are smooth, and the other four more or less coarsely reticulate in this species.

12 Hongkong; coll. R. Hungerford, Esq. [“at 10 fths."].

var. persoluta.

1 Japan; ex Mr. Damon [coll. A. Adams; labelled "C. reticulata"].

The last whorl is considerably less ventricose and more "solute" than in the original figure of the Singapore type! Long. $5 \frac{1}{2}$, diam. 3 mil.

\section{var. delicatula.}

2 Bombay; coll. Rev. S. B. Fairbank.

Rather smaller and of thinner texture; umbilicus a little less defined; apparently, one or two fewer of the spiral ribs.

17. Fossar [Couthouyia] striatulus.

Couthouyia striatula, A. Adams, P. Zool. S. 1863, Yobuko [Japan], 25 fths.

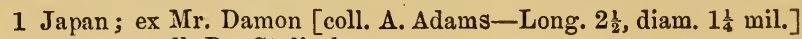

1 Arakan; coll. Dr. Stoliczka.

\section{Fossar [Couthouyia] decussatus.}

Couthouyia decussata, A. Adams, Ann. Mag. 1860, Mino-Sima.

1 Japan; ex Mr. Damon [coll. A. Adams].

Long $2 \frac{1}{2}$, diam. 1 mil. 


\section{Fossar [Couthonyia] pliciferus.}

Couthouyia plicifera, A. Adams, P. Zool. S. 1863, Yobuko (Japan).

1 Japan; ex Mr. Damon [coll. A. Adams].

Long. 2, diam. 1 mil.

20. Fossar [Couthouyia] appressus, n.sp.

2 Larek and Tumb Island (Persian Gulf); coll. W. T. Blanford, Esq.

A most beautiful and distinct form: long. 3, diam. 2 mil. Five whorls, the two nuclear ones smooth, the others minutely reticulate; a very prominent and characteristic raised keel below the suture of the last two whorls; the shell is unusually thick, the outer margin of the aperture patulously distended and reflected; the umbilical region widely but shallowly ex. cavated, bordered with a raised keel.

\section{Fossar [Couthouyia] styliferinus, n. sp.}

Three nucleolar apical whorls, smooth and very remarkable, resembling somewhat those of Stylifer; the other two whorls somewhat distantly spirally striate; last whorl very large and ventricose in proportion, umbilicus large, well defined.

Long. 3, diam. 2 mil.

1 Singapore (type); coll. C. J. Irving, Esq.

I found this specimen on a sp. of Malleus.

1 Bombay ; .coll. Rev. S. B. Fairbank.

var. [? dist. sp].

3 Singapore; coll. Dr. F. Stoliczka (perbaps not quite adult?)

\section{Fossar [Couthouyia] solutus, n. sp.}

Five whorls, apical two smooth, the others somewhat coarsely spirally striate; spire peculiarly attenuately pointed, pyramidical [not convex]; last whorl contracted, thoroughly "solute" below. Long. [vix] 3, diam. [vix] 2 mil.

1 Gwadar (Persian Gulf); coll. W. T. Blanford, Esq. 
23. Fossar [Couthouyia] subreticulatus, n. sp.

4 Tumb Island, Gwadar [type]; coll. W. T. Blanford, Esq.

3 Bombay; coll. Rev. S. B. Fairbank.

Resembling $F$. reticulatus, but with less developed umbilicus, fewer spiral ribs, \&c; three smooth nuclear whorls and three others cylindrically pyramidical, the last one only being ventricose.

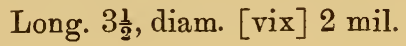

\section{Genus CONRADIA, A. Adams.}

Ann. Mag. V, 1860, p. 409, type C. cingulifera, A. Adams.

A. Adams also described a C. tornata, P. Z. S. 1863, from Gotto. There is a fossil species described as Turbo bayani, Bez. [1870].

\section{Conradia cingulifera.}

Conradia cingulifera, A. Adams, Ann. Mag. V. 1860, Mino. Sima at $63 \mathrm{fths}$.

1 Japan; ex Mr. Damon [coll. A. Adams].

Long. $3 \frac{1}{2}$, diam. 3 mil.

\section{Conradia clathrata.}

Conradia clathrata, A. Adams, Ann. Mag. T. 1860, Korea Straits at 63 fths.; = Fossarus adamsianus, Fischer, J.de C., XII, 1864, p. 259 (as it is not the Fossarus clathratus, Philippi).

1 Japan : ex Mr. Damon [coll. A. Adams].

long. $2 \frac{1}{2}$, diam, $2 \frac{1}{4} \mathrm{mil}$.

\section{Conradia pulchella.}

Conradia pulchella, A. Adams, Ann. Mag. 1861, Tsu-Sima at $16 \mathrm{fths.} \mathrm{and}$ Gotto at $48 \mathrm{fths}$.

1 Japau; ex Mr. Damon [coll. A. Adams].

Long. $1 \frac{3}{4}$, diam. $1 \frac{3}{4}$ mil. 


\section{Conradia stoliczkiana, n. sp.}

30 Singapore; coll. Dr. F. Stoliczka.

Long. $2 \frac{1}{2}$, diam. $2 \frac{1}{2}$ mil.

Of the usual " turbinate" shape, consisting of four whorls; the apical two are smooth, the third has two prominent raised spiral keels, coarsely, nodosely reticulated; the last whorl convexly ventricose, with eight spiral keels, nodosely granulated and with the interstices longitudinally striate; umbilicus more open than in the Japan species, peristome slightly fimbriate. Dr. Hungerford has dredged a closely allied form at Hongkong.

\section{Conradia adamsiana, n. sp.}

1 Oman, Persian Gulf [type]; coll. W. T. Blanford, Esq.

Long. 1, diam, 1 mil.

Turbinate, of $3 \frac{1}{2}$ whorls, the apical ones smooth, the last but one with two raised spiral keels, the last with five. I can detect no longitudinal striation whatever, the interstices consequently appear to be quite smooth; behind the peristome, which does not appear to be fimbriate, there are two conspicuous, thickened labial "varices"; the umbilicus open, much as in the preceding. Altogether a very distinct though so minute a form! The Red Sea specimen, which though also in good condition is probably scarcely adult, agrees exactly with the ,type, except in wanting the characteristic "labial varices" and in that the peristome is distinctly fimbriate; it also shows no signs (under the lens) of any longitudinal or reticulate sculpture. Named in honour of the late Mr. Henry Adams.

1 Annesley Bay; coll. W. T. Blanford, Esq.

\section{Conradia doliaris.}

Conradia doliaris, A. Adams, P. Zool. S. 1863, Mososeki at 7 fths.

2 Japan (typical); ex c. H. Adams, Esq.

Long. 3, diam. $2 \frac{1}{10}$ mil.

1 Hongkong; coll. R. Hungerford, Esq.

var. minor, nov.

2 Bombay ; coll. Rev. S. B. Fairbank.

2 Tumb Isl., Persian G.; coll. W. T. Blanford, Esq. 
Long. $2 \frac{1}{4}$, diam. $1 \frac{1}{2}$ mil.

After a long and careful examination under a lens, I can detect no difference whatever from the typical form, except its smaller size, possibly the last whorl is less ventricose and the aperture a trifle less everted; sculpture precisely similar.

$$
\text { Sub-Genus GOTTOINA, A. Adams. }
$$

P. Zool. S. 1863, types G. sulcifera and pyrgula, A. Adams.

\section{Conradia [Gottoina] pyrgula.}

Gottoina pyrgula, A. Adams, P. Zool. S. 1863, Gotto, at 48 fths.

1 Japan ; ex c. H. Adams, Esq.

Long. $2 \frac{1}{4}$, diam. 1 mil.

Not impossibly a Rissoid, near Alvania?

\section{Conradia [Gottoina], n. sp.}

1 Galle; coll. J. B. Baxter, Esq.

\section{? Genus RAULINIA, Mayer.}

J. de C., 1864, type Tornatella alligata, Desh. (upper tertiary foss.)

Raulinia badia, Tenison-Woods, P. Linn. Soc., N. S. W., Port Jackson (recent), is said to belong to this genus? M. Mayer also believes that Littorina monodonta, Desh. (chalk foss.), belongs here.

Not represented in the Museum.

[?] Genus Cithina, A. Adams.

P. Zool. S. 1863, types C. globosa, and C. spirata, A. Ad., as a Sub-genus of Conradia.

1. Cithna globosa [?].

Cithna globosa, A. Adams, P. Zool. S. 1863, Seto.Uchi, \&c.

1 Japan ; ex c. Dr. F. Stoliczka. 
Labelled " $C$. spiralis, A. Adams"; it cannot possibly be $C$. spirata, but agrees fairly with the description of $C$. globosa.

Long. 1, diam. 1 mil.

\section{2. [?] Cithna, sp.}

1 Japan; ex Mr. Damon (coll. A. Adams).

Labelled "Cithna omphalotropis, A. Ad." A very doubtful little form. Perhaps a Rissoid, or Jeffreysia?

\section{3. [?] Cithna, sp.}

1 Japan; ex Mr. Damon (coll. A. Adams).

Labelled "Cithna sinensis, A. Ad." The same applies to this; it might also be a Styliferina?

\section{4. [?] Cithna, sp.}

1 Japan; ex Mr. Damon (coll. A. Adams).

Labelled "Cithna consobrina, A. Ad." Probably a Rissoid?

Genus ISAPIS, H. and A. Adams.

Gen. Moll. I, 1864, type Narica anomala, C. B. Adams (Jamaica).

A. Adams has also described Isapis lirata [1860] and Isapis conoidea [1863] from Japan, and considers Narica ovoidea, Gould, and Isapis maculosa of Carpenter, both from Mazaltan, as also belonging to this section.

Genus FOSSARINA, Adams and Angas.

P. Zool. S. 1863, type F.patula, Adams and Angas.

\section{Fossarina variegata.}

Fossarina variegata, A. Adams (ubi?).

1 Japan (?) ; ex Mr. Damon (ex c. A. Adams).

\section{Fossarina patula.}

Fossarina patula, A. Adams and Angas, P. Zool. S. 1863, Port Jackson, "under stones."

1 Australia (?) ; ex Mr. Damon (ex c. A. Adams). 


\section{Fossarina picta.}

Fossarina picta, A. Adams, P. Zool. S., 1867, Japan, diam. 3, alt. $2 \frac{1}{2}$ mil.

2 Japan; ex Mr. Damon (ex c. A. Adams).

\section{Fossarina varia.}

Adeorbis varia, F. W. Hutton, Cat. Moll., New Zealand, 1873, Stewart's and Chatham Islands.

6 Chatham Islands; ex c. Dr. F. Stoliczka.

$$
\text { Sub-family LACUNIN } \nexists \text {, Stoliczka. }
$$

Genus CREMNOCONCHUS, W. T. Blanford.

Ann. Mag., 1869, type C. syhadrensis, Blf., for Cremnobates, Blf., op. cit. 1863 [not of Günther, 1861].

\section{Cremnoconchus conicus.}

Cremnoconchus conicus, W. T. Blanford, J. Asiat. Soc., Bengal, 1870, Torna.

12 Torna, near Poona; coll. Rev. S. B. Fairbank, and W. T. Blanford, Esq.

var. canaliculata.

C. syhadrensis, var. canaliculatus, W. T. Blanford, l. c., Torna.

7 Near Poona; coll. Rev. S. B. Fairbank, and W. T. Blanford, Esq.

var. edecollata.

12 Near Poona; coll. Rev. S. B. Fairbank.

I think it quite possible Stoliczka was correct, Proc. Asiat. Soc., Bengal, 1871, in uniting this species to the next as a variety only?

\section{Cremnoconchus carinatus.}

Anculotus carinatus, Layard, P. Zool. S. 1854, Mahabaleshwar; fide Stoliczka [1871] var.=C. conicus, Blf.; Blanford, J. A. S. B., 1870, pl.III,

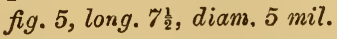

16 Mahabaleshwar; coll. Rev. S. B. Fairbank, and W. T. Blanford, Esq. 
var. gigantea.

3 Bombay Presidency; coll. Rev. S. B. Fairbank.

\section{Cremnoconchus syhadrensis.}

Cremnobates syhadrensis, W. T. Blanford, Ann. Mag., 1863, W. Ghâts,

[Bombay Pres.] at 2,000 ft.

30 Khandala, Poona, \&c. [Western Ghâts]; coll. Rev. S. B. Fairbank, and W. T. Blanford, Esq.

\section{Genus Stenotis, A. Adams.}

Ann. Mag., 1863, type S. laxata, A. Adams.

Issel has figured a Stenotis formosus from the Red Sea [subfossil].

\section{Stenotis laxata.}

Stenotis laxata, A. Adams, Ann. Mag., 1863, Japan, at $14 \mathrm{fths.}$

1 Japan; ex Mr. Damon [coll. A. Adams].

2. Stenotis [?] n. sp.

1 Bombay; coll. Rer. S. B. Fairbank.

3. Stenotis? sp.?

15 Hongkong; coll. R. Hungerford, Esq.

Genus LACUNA, Turton.

Type, Turbo puteolus, Turton, Zool. Journ. III, 1827; sect.=Epheria, Vélain, Faune St. Paul, 1880, type L. héberti, Vélain.

A. Adams has described a Lacuna carinifera from Borneo, P. Zool. S. 1851.

\section{Lacuna puteola.}

Turbo puteolus, Turton, Conch. Dict. 1819, England; fide Jeffreys = Cochlea parva, DaCosta, 1798, Helix fasciata, J. Adams, 1799, Helix lacuna, IItg. and Lacuna montacuti, Turton, Zool. Journ. III, 1827, \&c.; fide Jeffreys var. = Turbo auricularis, Mtg., 1805, and vars. conica, lactea, clausa and expansa [="L. puteola typical"].

4 North France; coll. Miss Beaufort. 


\section{Lacuna pallidula.}

Cochlea pallidula, DaCosta, Brit. Conch. 1778; fide Jeffreys var. albescens and var. patula, Thorpe, Brit. Mar. Conch. 1844.

2 England; ex c. G. Nevill, Esq.

var. neritoidea, Gould.

Lacuna neritoidea, Gould, Invert. Mass. 1841, "Chelsea Beach."

3 Maine [United States]; coll. Rev. E. R. Beadle.

$$
\text { var. patula. }
$$

Lacuna patula, Thorpe, Brit. Mar. Conch. 1844.

1 Falmouth; ex "Linnæa."

\section{Lacuna divaricata.}

Trochus divaricatus, Fabricius, Fauna Groenland, 1780 [not Trochus divaricatus of Lin.]; =Turbo vinctus, Mtg. 1805; fide Jeffreys, var. gracilior, Metcalfe.

4 England; ex c. G. Nevill, Esq.

2 Prince Edward's Island; coll. A. Nevill, Esq.

$$
\text { var. solidula. }
$$

Lacuna divaricata, var. solidula, Löven.

1 Greenland; ex "Linnæa."

var. quadrifasciata.

Turbo quadrifasciatus, Montagu, Test. Brit. Sup. 1805.

1 Long Island, United States; coll. Rev. E. R. Beadle.

var. canalis.

Turbo canalis, Montagu, Test. Brit. 1805.

1 England; ex c. Gr. Nevill, Esq.

\section{Lacuna crassior.}

Turbo crassior, Montagu, Test. Brit. 1805. 
2 Loc. ? ; ex c. G. Nevill, Esq.

1 Devonshire; ex "Linnæa."

\section{Lacuna variegata.}

Lacuna variegata, Carpenter, Ann. Mag. XIV, 1864, p.428, Neeah Bay, Vancouver District.

12 Monterey [California]; coll. Prof. E. C. Stearns.

\section{Lacuna unifasciata.}

Lacuna unifasciata, Carpenter.

20 Monterey; coll. Profs. W. Newcomb and E. C. Stearns.

Genus MODULUS, Gray.

Syn. Brit. Mus. 1849, type Trochus tectum, Gmelin.

\section{Modulus lividus.}

Trochus lividus, Philippi, Zeits. Mal. VI, 1849, p. 168, Hab.?

6 Panama; coll. Prof. E. C. Stearns.

\section{Modulus cerodes.}

Modulus cerodes, A. Adams, P. Zool. S. 1850, Mozanbique.

4. Gulf of California; coll. Prof. E. C. Stearns.

\section{Modulus floridanus.}

Modulus floridanus, Conrad, Amer. J. Conch. V, 1870, Florida.

8 Florida; coll. Profs. E. C. Stearns and W. Newcomb.

Sent as $M$. cancellatus, Lamarck, a species unknown to me and not recorded by Mörch, Malak. Blät. 1876, who seems to confirm Conrad's name.

3 Pine Key, Gulf of Mexico ; coll. Prof. E. C. Stearns.

\section{Modulus modulus.}

Trochus modulus, Linnaus, Syst. Nat. $X, 1758$, Hab.? = Trochus lenti. cularis, Chemnitz, 1781.

5 West Indies; ex c. Prof. W. Newcomb, and J. Wood-Mason, Esq. 
var. filosa.

Trochus filosus, Helbling, Privatg. Böhmen IV, 1779 (fide Mörch, 1876).

Mõrch does not record the following important locality.

2 Florida ; coll. Prof. E. C. Stearns.

\section{Modulus unidens.}

Trochilus unidens, Listeri, Chemn., Conch. Cab.I, Vol. X, p. 290, 1788 ; = Monodonta carchedonicus, Lam. 1822, Hab.?; fide Mörch=Trochus perlatus, Dillwyn, also Wood (not of Gmelin), and var. minor = Monodonta angulata, C. B. Adams, P. Bost. S. 1845, Jamaica.

7 Panama and Gulf of Fonseca; coll. Profs. E. C. Stearns and W. Newcomb.

\section{Modulus disculus.}

Trochus disculus, Philippi, Zeits. Malak. 1846, Mazatlan.

\section{var. [fide Newcomb].}

20 Gulf of California; coll. Profs. E. C. Stearns and W. Newcomb.

\section{Modulus obtusatus.}

Trochus obtusatus, Philippi, Conch. Cab. II, 1846, Los.?, for Chemnitz, fig. $1569-70$.

7 Mauritius and Ceylon; coll. G. Nevill, Esq.,

Despite the highly valued opinion of von Martens, I consider this a distinct species.

1 Rodriguez; coll. J. Caldwell, Esq.

A doubtful specimen; in very bad condition.

\section{Modulus tectum.}

Trochus tectum, Gmelin, Syst. Lin. 1788, West Indies; from Madagascar, Natal, Red Sea, Bourbon, fide v. Martens; fide Petit $=$ Monodonto retusa, Bruguiere = Modulus cidaris, Reeve.

7 Mauritius and Seychelles; coll. G. Nevill, Esq.

3 Natal ; coll. G. Nevill, Esq.

3 Trincomalee (?); ex c. Dr. F. Stoliczka. 


\section{Modulus candidus.}

Modulus candidus, Petit, J. de C., IV, 1853, Pacific Ocean [?]; fide McAndrew, Sandwich I. and Suez.

4. Andamans; coll. Capt. E. C. Temple, and J. Wood-Mason, Esq.

6 Ceylon; coll. G. Nevill, Esq.

Though undoubtedly very close to the preceding, I have seen no intermediate specimens; the former I have not seen from the Mascarene Province nor the latter from the Andamans.

4 N. Caledonia; ex c. P. Bouvier, Esq.

\section{Modulus obliquus [?].}

Modulus obliquus,, A. Adams, Ann. Mag., VIII, 185̃1, Red Sea.

1 Aden; coll. Dr. F. Stolcizka.

In such bad condition as to be scarcely recognizable. It looks like a rather distinct form?

7. Family PLANAXIDÆ, H. \& A. Adams.

Subfamily LITIOPIN AE, Gray.

Genus LITIOPA, Rang.

Ann. Sci. Nat. XVT, 1829, p. 303, type L. melarostoma, Rang; fide A. Adams $=$ Bombycinus, Bélanger and Lesson.

\section{Litiopa bombax.}

? L. maculata, Rang, l. c. 1829, Atlantic Ocean.

5 Atlantic, on sea-weed; ex c. Prof. W. Newcomb.

3 Bahamas; ex c. C. F. Ancey \& P. Bouvier, Esqrs. (as "L. bombyx, Rang"). 


\title{
2. Litiopa n. sp.
}

10 Off the S. E. coast of Arabia ; coll. W. T. Blanford, Esq.

Spine very acuteìy produced.

\section{Litiopa ventrosa [?].}

Litiopa ventrosa, A. Adams, Ann. Mag., VIII, 1861, Indian Ocean.

10 Oman \& Gwadar (Persian Gulf); coll. W. T. Blanford, Esq.

\author{
var. minor (? dist. sp.)
}

4 Gwadar; coll. W. T. Blanford, Esq.

\section{Genus STYLIFERINA, A, Adams.}

Ann. Mag., VI, 1860, types S. orthochila, A. Ad., and S. goniochila; emend.

l. c. 1862, as subgenus of Alaba

\section{Styliferina savignyi.}

Litiopa savignyi, Issel, Malac. Mar. Rosso. 1869, Suez-long. 3, diam. $1 \frac{1}{2}$ mil.; Descr. de l'Egypte, pl. III, fig. 19 [male].

2 Mauritius ; coll. G. Nevill, Esq.

2 Aden \& Muscat; coll. Ir. F. Stoliczka, and W. T. Blanford, Esq.

4 Suez [typical; ; coll. Prof. A. Issel.

\section{Styliferina goniochila.}

Styliferina goniochila, A. Adams, Ann. Mag., I'I, 1860, and VIII, 1862, Jüan.

1 Annesley Bay ; coll. W. T. Blanford, Esq.

30 Mauritius; coll. G. Nevill, Esq.

3 Japan; ex Mr. Damon (coll A. Adams).

2 Seychelles \& Andamans; coll. G. Nevill, Esq.

$$
\text { var. (? dist. sp.) }
$$

1 Hongkong; coll. R. Hungerford, Esq.

Shorter spire, less ovately elongate last whor], which appears to be almost subangulate. 
3. Styliferina [?] sp. [?]

1 Singapore; coll. Dr. F. Stoliczka.

Of very doubtful generic determination.

\section{Styliferina sp. ?}

3 Andamans; coll. G. Nevill, Esq.

Whorls subangulate.

5. Styliferina sp.?

3 Andamans; coll. G. Nevill, Esq.

Whorls convexly rounded.

1 Mauritius; coll. G. Nevill, Esq.

var. (? dist. sp.)

2 Mauritius; coll. G. Nevill, Esq.

The last whorl conspicuously more produced

\section{Styliferina? sp.}

2 Mauritius; coll. G. Nevill, Esq.

Found together in an empty oyster shell on the shore.

\section{Styliferina? sp.}

7 Hongkong; coll. R. Hungerford, Esq.

\section{Genus DIALA, A. Adams.}

A.nn Mıg., VIII, 1861, type D. varia, A. Ad.; Smith. P. Zool. S., 18'5, p. 538 .

There are also $D$. tenuis and simplex, Smith, 1875, from Japan; $D$. succincta, A. Adams, from Suez; and D. suturalis, Philippines and from the Suez.

\section{Diala sulcifera.}

Diala suleifera, A. Adams, Ann. Mug., X, 1862, Japan. 
Long, $2 \frac{1}{T^{1}}$, diam. $\frac{3}{4}$ mil.

2 Japan; ex Mr, Damon (coll. A. Adams).

6 Hongkong; coll. R. Hungerford, Esq.

12 Bombay; coll. Rev. S. B. Fairbank.

20 Persian Gulf; coll. W. T. Blanford, Esq,

\section{Diala lauta.}

Diala lauta, A. Adams, Ann. Mag., X., 1862, Adelaide.

1 Loc.? ; ex Mr. Damon (coll. A. Adams).

1 Loc.? ; ex c. G. Nevill, Esq.

\section{Diala macula.}

Alaba macula, Recluz, fide Schwartz, in litt. and =A. semistriata, Philippi, Zeits. Mal., 1849, p. 34, Aden and Deser. de l'Egypte,,$l . I I I ;=D$. varia, A. Adams, Ann. Mag., VIII, 1861, China and Japan (Suez, fide Mc.tndrew).

3 Japan ; ex Mr. Damon (coll. A. Adams).

50 Mauritius, Natal, Andamans, Seychelles; coll. G. Nevill, Esq.

30 Suez, Aden, Annesley Bay and Persian Gulf; coll. W. T. Blanford, Esq.

50 Bombay, Arakan, Nicobars; coll. Dr. F. Stoliczka.

8 Roweiah, Red Sea, and Galle; coll. J. B. Baxter, Esq.

4. Hongkong; coll. R. Hungerford, Esq.

The figure in the Descr. de l'Egypte agrees better with the Japanese specimens and Adams' description of $D$. leucosticta than with the Mauritian form.

\section{Diala n. sp.}

20 Nicobars ; coll. Dr. F. Stoliczka.

\section{Diala sp.?}

4 Kathiawar; coll. F. Fedden, Esq.

\section{Diala sp.}

2 Arakan; coll. Dr. F. Stoliczka.

\section{Diala (?) sp.}

1 Bombay; coll. Rev. S. B. Fairbank. 


\section{Diala sp.}

3 Mauritius and Singapore; ex c. Dr. F. Stoliczka.

\section{Diala sp.}

2 Annesley Bay; coll. W. T. Blanford, Esq.

Of a brilliant uniform green coloration.

\section{Diala marmorea.}

Diala marmorea, Carpenter.

10 Monterey, California; coll. Prof. E. C. Stearns.

\section{Genus ALABA, $\#$. and A. Adams.}

Gen. Moll. I, 1854, type A. melanura, C. B. Adams; Smith, P. Zool. S. 18\%5, p.538; fide A. Adams = Tuberia, Carpenter.

A. Adams described A. lucida and A. imbricata, both from Japan and Suez; the latter is rather a sp. of Diala, fide Smith.

\section{Alaba felina [?]}

Alaba felina, A. Adams, Ann. Mag. X, 1862, Japan.

1 Japan; ex Mr. Damon [coll. A. Adams; labelled "A. felina"].

I very much doubt the correctness of this identification.

\section{Alaba leucosticta.}

Diala leucosticta, A. Adams, Ann. Mag., VIII, 1861, Shantung and Loshan. kow.

Long. 6, diam. 3 mil.

1 China? ; ex Mr. Damon [coll. A. Adams].

\section{Alaba melanura.}

Rissoa melanura, C. B. Adams, Contr. Conch. 1850, p. 116, Jamaica.

1 Loc. ?; ex Mr. Damon [coll. A. Adams].

12 W. Indies; ex c. J. Wood-Mason, Esq. 
4. Alaba sp.

1 Japan; ex Mr. Damon [coll. A. Adams; labelled "A. subulata, A. Ad."’].

\section{Alaba subangulata.}

Alaba subangulata, A. Adams, Ann. Mag. X, 1862, Tsu-Sima at 16 fths.

Long. $6 \frac{1}{2}$, diam. 3 mil.

1 Japan; ex Mr. Damon [coll. A. Adams].

\section{Alaba woodmasoniana, n. sp.}

5 Andamans; coll. G. Nevill, Esq.

One of the most interesting species yet discovered, something like the preceding.

var. [? dist. $8 \mathrm{p}$.

1 Andamans; coll. J. Wood-Mason, Esq.

3 Andamans; coll. Dr. F. Stoliczka.

\section{Alaba cornea [?]}

Diala cornea, A. Adams, Ann. Mag. 1861, VIII, Hulu-Shan Bay, \&. .

1 China [?]; ex Mr. Damon [coll. A. Adams].

This identification seems to me very doubtful.

\section{Alaba picta.}

Diala picta, A. Adams, Ann. Mag. VIII, 1861, Shantung, \&c.

Long. $5 \frac{3}{4}$, diam. $2 \frac{1}{2} \mathrm{mil}$.

2 China ; ex c. H. Adans, Esq.

2 China [?]; ex Mr. Damon [coll. A. Adams].

The original_description is very bad. 
9. Alaba n. sp.

3 Balapiti, Ceylon; coll. G. Nevill, Esq.

One of the most beautiful species I know.

var. [? dist. sp.]

1 Ceylon; coll. G. Nevill, Esq.

\section{Alaba blanfordi.}

Alaba blanfordi, A. Adams, Ann. Mag. X, 1862, "Siam [?], coll. W. T: B".

20 Cape Negrais, Arakan [typical]; coll. W. T. Blanford, Esq.

1 Loc. ? ; ex Mr. Damon [coll. A. Adams].

40 Bombay, Coromandel, "Yuri, and Andamans; coll. Dr. F. Stoliczka, H. F. Blanford and H. Raban, Esqrs.

var.

9 Hougkong [at 6 Fths.]; coll. R. Hungerford, Esq.

Scarcely separable even as a variety.

11. Alaba n. sp.

10 Akyab; coll. Dr. F. Stoliczka.

In very bad condition.

\section{Alaba doriæ.}

Alaba doriæ, Issel [ubi ?].

5 Suez; coll. Prof. Issel [sent as " $A$. dorice, Issel "].

12 Annesley Bay; coll. W. T. Blanford, Esq.

The latter in bad condition, apparently two species confused.

\section{Alaba sp.}

6 Port Natal ; coll. (ł. Nevill, Esq.

A fine large form, near $A$. blanfordi, unfortunately in bad condition. 


\section{Alaba sp.}

3 Tawila [Persian Gulf]; coll. W. T. Blanford, Esq.

\section{Alaba zebrina [?]}

Diala zebrina, A. Adams, Ann. Mag. VIII, 1861, Japan, also Philippines.

1 Singapore; coll. Dr. F. Stoliczka.

? var. [? dist. sp.]

2 Hongkong; coll. R. Hungerford, Esq.

It is just possible these may prove to be worn specimens of the form previously noted as A. blanfordi, var., though I think they are distinct; unfortunately, they are in bad condition.

\section{Genus PLANAXIS, Lamarck.}

Hist. Anim. s. Vert. VII, 1822, type Pl. sulcatus, Born; sect.=Hinea, Leach ms., Gray, P. Zool. S. 1845, type Pl. mollis, Sow.

A. Adams, P. Z. S., 1851, described a $P l$. zonatus from the Philippines, and a $\mathrm{Pl}$. cingulata from China.

\section{Planaxis sulcatus.}

Buccinum sulcatum, Born, Test. Mus. Vindob, 1780; =Pl. buccinoides, Desh.; = Buc. pyramidale, Gmelin; $=P l$. undulata, Lam. ; fide Martens, from Madagascar ana' Seychelles, \&c., os Pl. pyramidalis ["scarce. ly separable from W. Indian Pl. sulcatus"]; Japan, fide Lischke.

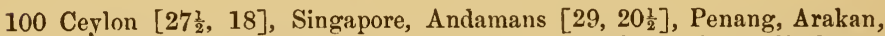
Bombay and Mauritius; coll. W. T. Blanford, F. Stoliczka, J. Wood-Mason, and G. Nevill, Esqrs.

2 N. Caledonia ; ex c. P. Bouvier, Esq.

12 Hongkong; coll. R. Hungerford, Esq.

\section{subvar. contracta.}

2 Ceylon; coll. G. Nevill, Esq.

subvar. nana.

6 Vizagapatam; ex c. Madras Museum.

3 Australia [?]; ex c. Dr. F. Stoliczka. 
subvar. rugosolirata.

3 North Australia; ex c. British Museum.

I cannot accept $P l$. undulatus, Lamarck, even as a variety, because some individuals in the same "hand-full " are of this, while others are of the ordinary coloration. It is interesting to find this species at Bombay, adjoining the babitat of the next form.

\section{Planaxis savignyi.}

Planaxis savignyi, Deshayes, Mag. Zool. 1844, pl. 109, Red Sea and Marla. gascar, long. 22, diam. 8 mil.; fide Issel, Pl. griseum, Brocchi, Cat. Conch. G. Forni, 1822, G. of Suez.

50 Persian Gulf; coll. W. T. Blanford, Esq.

A trifle smoother and more ventricose than in the typical figures; the spots are often undulately confluent, as in the preceding species. Long. 21, diam. 16 mil.

6 Suez; ex c. Asiatic Soc. Bengal.

Agree exactly with Savigny's figure.

20 Massaua and Annesley Bay; coll. Prof. A. Issel, and W. T. Blanford, Esq.

A smaller and shorter-spired form closely resembling the Arakan variety in shape. McAndrew records both $P l$. griseus and $\mathrm{Pl}$. savignyi as distinct species, both as frequent at Suez !

var. subnigra.

12 Hormuz and Muscat, Persian Gulf; coll. W. T. Blanford, Esq.

20 Aden; coll. G. Nevill, Esq. [mostly young].

2 Kathiawar; coll. F. Fedden, Esq.

var. encaustica.

Planaxis encausticus, Smith, Ann. Mag. IX, 1872, Arakan, long. 20, diam. 12 mil.

10 Arakan [typical]; coll. W. Theobald, Esq. 
If Mr. Smith had been able to examine as many specimens as I have, he would have found that none of the characters he relies on are constant; e.g., it is not smoother than many of the Persian Gulf specimens; the sulci are of exactly the same width; and the coloration recorded is not constant. Curiously the typical coloration is also exactly represented by typical Persian $P l$. breviculus. The "basal canal" likewise is often, if not generally, entirely like that of its allies !

The habitat is very interesting, being the only one known East of the Bay of Bengal : it is particularly important to find the species actually living with $P l$. sulcatus. The fig. of $P l$. encausticus in the Con. Iconica is very fair, but fig. 30 , a " $P l$. savignyi," has the last whorl less tumid than is usually the case.

\section{Planaxis breviculus.}

Planaxis breviculus, Deshayes, Mag. Zool. 1814, Hab.?, long. 21, diam. 15 mil.; Suez, "frequent," fide McAndrew.

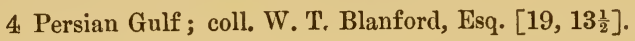

Coloration close to that of the original figure, also to that of the Arakanese Pl. savignyi, var. encaustica. Pl. breviculus is scarcely more than a well-marked variety of $P l$. savignyi? The darker-coloured aperture, however, with obsolete liration, seems to be characteristic.

var. tessellata, nov.

Pl. breviculus, var., Smith, Ann. Mag. IX, 1872, Persian Gulf.

20 Persian Gulf; coll. W. T. Blanford, Esq. [long. 20, diam. 14].

Both these and the preceding have a more acuminate spire than in the figure of Deshayes. The figure in Con. Iconica seems to me different again; possibly it represents $P l$. brevis, Quoy, as Sowerby quotes the latter's localities-"Guam and N. Guinea"-for the figure in question.

\section{Planaxis planicostatus.}

Planaxis planicostatus, Sowerby, App. Tankerv. Cat. 1825, Gallapagos I.; fide Smith=Buc. planaxis, Wood; $=P l$. canaliculata, Duval, Rev. Zool. Cuv. 1840; =Pl. circinata, Lesson, Rev. Zool. Cuv. 1842, "Acapulco 0.020."

5 Panama; ex c. Prof. E. C. Stearns and Asiatic Soc. Bengal $[28,16]$. 
Agree with Con. Iconica, Pl. IV, fig. 26.

subvar. minor.

10 Panama; ex c. Prof. W. Newcomb $\left[16 \frac{1}{2}, 9 \frac{1}{2}\right]$.

subvar. brevispira.

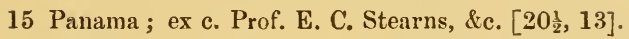

\section{Planaxis nigritellus.}

Planaxis nigritellus, Forbes, P. Zool. S. 1850 , Straits of S. Juan del Fuaco; $=P l$. acutus, Menke, Zeits. Mal. 1850 [not of Krauss].

6 Gulf of California; coll. Prof. E. C. Stearns.

The last whorl and aperture are, proportionately, considerably less elongate than in the figure given by Forbes.

\section{Planaxis lineolatus.}

Planaxis lineolatus, Gould, P. Bost. S. III, 1849, Wilson's Island; var. = Pl. strigatus, Sow., Conch. Icon. 1878 [as of Hanley, ms. Pacific]; Tahiti and Samoa, fide Gräffe; Bourbon, fide Deshayes.

7 Paumotu; coll. H. Pease, Esq.

Two of these are dark-coloured and many-striped; four white, with three narrow bands on last whorl; one uniform white! Long. 9, diam. 5 mil.

3 New Caledonia : ex. c. P. Bouvier, Esq. $\left[8 \frac{1}{4}, 4\right]$.

3 Mauritius; ex c. Dr. F. Stoliczka.

In very bad condition; identification therefore questionable.

\section{var. producta.}

Smooth throughout; many banded; aperture rather large, "subpatulous."

1 Ceylon; coll. G. Nevill, Esq. $\left(4 \frac{1}{2}, 2 \frac{1}{4}\right)$.

1 Aden; coll. Dr. F. Stoliczka $(6,3)$.

15 Cherboniane Reef; coll. 1)r. Armstrong.

20 Andamans; coll. J. Wood-Mason and G. Nevill, Esqrs, 
More attenuately produced and less distinctly angulate than the preceding specimens. Long. $5-4 \frac{3}{4}$, diam. $2 \frac{1}{4}$ mil. It is possibly the Planaxis succinctus of McAndrew from Suez? Through the kindness of Colonel Godwin-Austen, I have had the advantage of Mr. Edgar Smith's opinion of one of the Cherboniane Reef specimens-he considers it as Planaxis lineolatus, Gld.

\section{Planaxis ineptus.}

Planaxis inepta, Gould, P. Bost. S. VII, 1860, Kikaia Bay-" axis 4, diam. 2.5 mil."

12 Cherboniane Reef, Arakan; coll. W. T. Blanford, Esq.

12 Ceylon; coll. G. Nevill, Esq.

Long. 8, diam. $4 \frac{1}{4}$, another $4 \frac{1}{2}, 2 \frac{1}{2}$ mil.; more or less sulcate, last whorl always more or less angulate; aperture contracted and not "subpatulous" as in Pl. lineolatus; very variable in size as will be seen from the above measurements, generally striped as in the figure of the Corich. Iconica of $\mathrm{Pl}$. zonatus, A. Adams, [Philippines; smooth], sometimes only one, sometimes many banded. It may be an extreme var. of Pl. lineolatus, Gld.? My identification has been confirmed by Mr. Edgar Smith, of the British Museum.

$$
\text { var. persulcatula. }
$$

1 Ceylon; coll. G. Nevill, Esq.

Perhaps an abnormal dwarf? Long. 4, diam. 2, mil.

$$
\text { var. virgata. }
$$

Planaxis virgatus, Smith, Ann. Mag. IX, 1872.

50 Andamans and Arakan; coll. Captain R. C. Temple, W. T. Blanford and G. Nevill, Esqrs.

Long. 5 mil. Mr. Smith has identified one of these specimens as his $P l$. virgatus.

\section{Planaxis eboreus.}

Planaxis eboreus, Smith, Ann. Mag. IX, 1872, W. Indies.

2 W. Indies; ex c. J. Wood-Mason, Esq. 


\section{Planaxis lineatus.}

Buccinum lineatum, DaCosta, Brit. Conch., 1776, West Indies;=Buc. pediculare, Lam. and Kiener.

30 West Indies; ex c. J. Wood-Mason and G. Nevill, Esqrs.

var. succincta.

Planaxis succinctus, A. Adams, P.Zuol. S., 1851, Peru and the W. Indies; Suez, fide Mc.Andrew.

16 Guadaloupe; ex c. P. Joly, Esq.

12 West Indies; ex c. Prof. W. Newcomb.

\section{Planaxis labiosus.}

Planaxis labiosus, A. Ad., P. Zool. S. 1S5̃1, Sandwich Islands; Don. Bism. pl. II, fig. $12 ;=P l$. bronni, Dunker, Mal. Bl.1862, Austr. Ocean, long. 12, diam. 6 mil.

15 Sandwich Islands; coll. H. Perse, Esq. [as "Pl. labiosus] and Prof. W. Newcomb $\left[11,6 \frac{1}{2}\right]$.

var. plumbea.

Pl:ınaxis plumbeus, Pease, P. Zool. S. 1861, Sandwich Islands.

4 Sandwich Islands [typical of Pl.plumbeus ; 13, F. $\frac{1}{2}$; ]; coll. H. Pease, Esq.

var. striatula.

Planaxis striatulus, Philippi, Zeits. Mal. 1S51; Don. Bism.pl. II, fig. 11.

5 Loc.? ; ex c. British Museun [as " P'l. striatulus, Phil."]

I presume these have been correctly identified by Mr. Smith as Philippi's species? However that may be, these specimens are certainly only a slight variety of $P l$. labiosus, distinguished by slightly smaller size-long. 10, diam. $5 \frac{1}{4}$-more contracted aperture, \&c. Probably Philippi has priority ?; von Martens seems to consider the two species as scarcely separable, comp. Don. Bism., p. 42.

\section{Planaxis suturalis.}

I lanaxis suturalis, Smith, Ann. Mag, IX, 1872, China Seas.

20 Ceylon and Andamans; coll. G. Nevill, Esq., and Colonel E. I. Berkeley [anfr. 7, long. 5, diam., 21: 


\section{Planaxis ater.}

Planaxis ater, Pease, Amer. Journ. Conch. T, 1868, Marquesas-long. 8, diam $4 \frac{1}{2}$ mil. [not Pl. ater of the Con. Icon.]; = Pl. hanleyi, Smith, Ann. Mag. XII, 1872, Sandwich Islauds, long. 12, diam. $5 \frac{1}{2}$ mil.

1 Viti ; ex Mus. Godeffroy.

Agrees excellently with original figure, \&c.

\section{Planaxis atropurpureus.}

Planaxis atropurpureus, Recluz, Rev. Zool. Cuv. 1843, p. 261, South Seasanfr. 5 to 6-long. ‘, diam. 4 mil.; ?=Pl. albersi, Dkr., Nov. Conch. Sup. II, pl. II, fig, 3̃̄-37, W. Africa.

2 Paumotu; ex Mus. Godeffroy.

Sent as "Pl. brevis, Quoy" [Guam and N. Guinea], which is, however, quite different. It is like a dwarf $P l$. abbreviatus.] Long. 5, diam. 3 mil. ; anfr. 3.

\section{Planazis semisulcatus.}

Planaxis semisulcatus, Sowerby, Genera Shells, I, 1824, Hab. ?;=Purpura nucleus, Lam. VII, 1822, [not Buc. nucleus, Brug. 1789, from Madagascur].

12 W. Indies, ex c. Prof W. Newcomb.

1 St. Thomas; ex c. Colonel Godwin-Austen.

\section{Planaxis acutus.}

Planaxis acutus, Krauss, Sïd-Afrik. Moll. 1848, Natal, long. 6, điam. $3 \frac{1}{3}$ lin. ; var. = Pl. similis, Smith, Ann. Mag. XII, 1872, sandwich Islands, long. $11 \frac{1}{2}$, diam. $5 \frac{1}{2}$ mil.

The figures 11 and 12 of the Con. Iconica, " Pl. simitis and Pl. hanleyi, Smith," both resemble more closeiy the original figure of Krauss than does fig. 9, "Pl. acutus". I consider Smith's $P l$. similis doubtfully separable even as a variety. The species varies much in size, and tendency to subangulation at the neriphery. $P l$. ater, Pease, is scarcely more than a geographical race or variety.

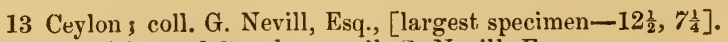

10 Mauritius and Bourbon; coll. G. Nevill, Esq.

1 Andaunans [?]; coll. Dr. F. Stoliczka. 
Possibly may have got mixed; it was amongst a lot of $P l$. nicobaricus?

var. similis.

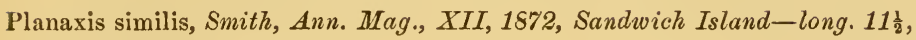
diam. $5 \frac{1}{2}$ mil.

6 Bombay; coll. Rev. S. B. Fairbank.

Very variable; sometimes attains a relatively large size-viz., long. $16 \frac{1}{2}$, diam. 9 mil.

\section{Planaxis nicobaricus.}

Planaxis nicobaricus, von Frauenfeld, Moll. Novara Exp. 1866, Nicobarslong. 12, diam. 8 mil., anfr. 6 ; Con. Iconica, fig. 13, as Pl. niger [not Pl. niger, Quoy].

Prof. von Martens, Zool. Record for 1866, p. 182, says of this species-" Most closely allied to $\mathrm{Pl}$. nucleus [Brug.] and $P$. ovatus [Krauss]."

There seems to be considerable confusion about this form. Sowerby figures it excellently as above, but confused it with a species of Quoy's not half its size, and consisting of four whorls only. Von Frauenfeld's description and figure do not at all well represent the species. I imagine they were taken from a dead and worn specimen, in bad condition.

Whorls either six or seven, the upper ones perfectly smooth and of lighter colour, the last two under the lens obsoletely striate, the last of all so much as to be almost smooth, except for an incised infrasutural girdle, and some five or six broad sulcations quite at the base. A good characteristic is the unusually sharp pointed apex. The shell varies from a rich chocolate colour to black and is covered with a rather thick, compact, lighter coloured epidermis. The aperture is remarkably expanded or "patulous," of a uniform rich brown colour, not margined at peristome, denticulations within so fine as to be often almost imperceptible even with a leas.

Long. 12 to 14 , diam. 7 to $7 \frac{1}{2}$ mil.

3 New Caledonia; ex c. P. Bouvier, Esq.

15 Andamans and Nicobars; coll. F. A. de Roepstorff and Dr. F. Stoliczka. 
These are of a rich brown colour and consist of seven whorls ; spire more acuminate than in the next form.

7 Maré, Loyalty Islands; ex c. F. Layard, Esq.

It was, I suspect, one of the specimens from this locality, out of Mr. Hanley's collection, that was figured by Sowerby in the Con. Iconica? They are almost quite black, of six whorls; two have a peculiarly shortened spire and may belong to a distinct form.

Long. 12, diam. 7 mil.

1 Camorta, Nicobars; coll. F. A. de Roepstorff, Esq.

Long. 10, diam. $5 \frac{1}{2}$ mil.

Quite black, of six whorls; exactly resembles Sowerby's fig. 13.

\section{Planaxis niger.}

Planaxis niger, Quoy, Voy. de l'Astrolabe, 1832, New Ireland, anfr. 4long. 4, diam. 2 lin., "spire courte, obtuse, corrodée, 3-4 stries à la base" ;=P. abbreviatus, Pease, P. Zool. S., 1865, Central Pacificlong. 10, diam. 6 mil.

It would almost seem as if the description and figures of Quoy applied to two distinct species, the former undoubtedly referring to this species and the latter resembling more closely the preceding (except the line denoting natural size, which refers to the former)?

5 Tahiti; coll. H. Pease (typical Pl. abbreviatus) and ex Mus. Godeffroy. 1 Arakan; coll. R. Hungerford, Esq.

Long. $8 \frac{3}{4}$, diam. 5 mil.

Spire short, obtuse, and corroded; four whorls, 3-4 sulcations at base of the last whorl; jet black, with aperture much as in preceding specimens, denticulations being almost obsolete, but a good deal more developed than in Tahiti specimens, which also have the last whorl more tumid; tooth above columella remarkably developed in this specimen, much as in fig. 12 of Con. Iconica. 


\section{Planaxis mollis.}

Planaxis mollis, Sowerby, Genera Shells, I, fig. 2, 1824, Hab.? ; = Bucinum brasilianum, Lam. Hist., VII, 1822 [misnomer];= Buccinum lavigatum, Wood, Ind. Test. Sup. 1828 ; fide Smith var.=Pl. fulva, A. Adams, P. Zool. S. 1851, p. 271, Swan River; var.=P. pigra, Forbes, P. Zool. S. 1850 , Pitcairn Island.

9 Port Jackson, \&c.; coll. Dr. J. C. Cox and ex c. Asiatic Soc. Bengal.

3 Australia; ex c. Dr. F. Stoliczka.

\section{Genus QUOYIA, Deshayes.}

Enc. Méth. II, 1830 [sine descr.] type Planaxis decollata, Quoy, and of Gray, Syn. Brit. Mus., 1840 ; = Fissilabria, Brown, Edinb. Journ. N. H. 1836 ; = Quoya, Deshayes, Enc. Méth., III, 1843 ; =Leucostoma, Swainson, Treat. Malac., 1840.

\section{Quoyia decollata.}

Planaxis decollata, Quoy, Voy. Astrolabe, 1832, New Guinea; Caroline Islands fide v. Martens, New Caledonia, fide Fischer, and Yap, fide Schmeltz.

1 Moluccas; ex c. Dr. H. Dohrn.

1 Caroline I. ; ex c. C. H. Ancey, Esq.

Genus HOLCOSTOMA, H. and A. Adams.

P. Zool. S. 1853 and Gen. Moll. I, p. 324, 1854, type H. piligerum, Philippi, [from Mascarene Islands, fide Deshayes, \&c.].

I have never been able to obtain a specimen of this interesting genus.

\section{Family MELANIIDE, Orbigny.}

Moll. Canar. 1837 ; = Melaniada, Gray, 1840.

Sub-family CERIPḦASIN E, Gill.

P. Acad. Nat. Sci. Philad. 1863 [Febr.];=Family Strepomatida, Halde. man, l. c. [Sept.]

I am unable to agree with Messrs. Brot and Tryon in accepting Haldeman's namie in preference to that of Gill's. 


\section{Genus IO, Lea.}

Philos. Trans, II, 1832, type Io fluviatilis, Say; fide Brot., Sub-Genus of Pleurocera.

\section{Io fluvialis.}

Fusus fluvialis, Say, J. Acad. Nat. Sci., , 1925.

\section{var. inermis.}

Io inermis, Anthony, P. Acad. Nat. Sci., 1860.

2 Tennessee; coll. Prof. W. Neẃcomb.

\section{Io spinosa.}

Io spinosa, Lea, Philos. Trans. V, 1834, Kolston Rv. [Virginia].

2 Tennessee; coll. Prof. W. Newcomb.

\section{Genus PLEUROCERA, Rufinesque.}

J. de Phys. Brux. 1819 [no type recorded];=Cerithasia, Swainson, 1840, type C. sulcata; = Trypanostoma, Lea, 1S62, type Mel. canaliculata, Say $;=$ Strepoma, Auct. [as of Rafin.].

Fide Tryon a sub-genus of Io.

\section{Pleurocera canaliculata.}

Melania canaliculata, Say, J. Acad. Nat. Sci., MI, 1821.

4 Indiana; coll. I. Lea, Esq. [Asiat. Soc. Bengal].

2 Ohio ; coll. Prof. W. Newcomb.

3 Coosa Rv., Alabama ; coll. lievd. E. R. Beadle [labelled "M. ponderosa"].

\section{Pleurocera ponderosa.}

Melania ponderosa, Anthony, P. Acad. Nat. Sii. 1560.

5 Indiana; ex c. Dr. T. Oldham.

\section{Pleurocera filum.}

Melania filum, Lea, Philos. Proc. II, 1S45, Alabama.

8 'Tennessec; coll. Prof. W. Newcomb. 


\section{Pleurocera prasinata.}

Melania prasinata, Conrad, Amer. J. Sci. V, 1834.

4. Consa Rv., Alabama ; coll. Rev. E. R. Beadle.

\section{Pleurocera annulifera.}

Melania annulifera, Conrad, New freshwater Sỉells, 1834.

2 Alabama; coll. Prof. W. Newcomb.

\section{Pleurocera clarkii.}

Melania clarkii, Leu, P. Acud. Nat. Sci., 1862, "Duck Creek, Tennessee." 2 Alabama; coll. Prof. IV. Newcomb.

\section{Pleurocera elevata.}

Melania elevata, Say, J. Acad. Nat. Sci. II, 1821.

$$
\text { var. elongata. }
$$

Melania elongata, Lea, Philos. Trans. IT, 1S32, West Tennessee.

2 Alabama; coll. Prof. W. Newcomb.

\section{Pleurocera troostii.}

Trypanostoma troostii, Lea, P. Acad. Nat. Sci, 1862, Tennessee, Alabama, and Indiana.

4 Alabama; coll. Prof. W. Newcomb.

\section{Pleurocera monilifera.}

Trypanostoma moniliferum, Lea, $P$. Acad. Nat. Sci., 1862, Tennessee, Indiana, \&.c.

2 Alabama; ex c. Prof. W. Newcomb;

\section{Pleurocera lesleyi.}

Trypanostoma Lesleyi, Lea, P. Acad. Nat. Sci. 1861,-East Tennessee and Kentucky.

1 Kentucky; coll. Prof. WV. Newcomb. 


\section{Pleurocera prestoniana.}

Trypanostoma Prestoniana, Lea, P. Acad. Nat. Sci. 1864, p. 3 [foot-note], for M. vanuxemii, l. c. 1861 [not M. vanuxemiana, Lea].

7 Ringgold, Georgia; coll. Rev. E. R. Beadle.

\section{Pleurocera subularis.}

Melania subularis, Lea, Philos. Trans. ID, 1832, "Niagara River."

16 Mohawk Rv., New York and Michigan; coll. Rev. E. R. Beadle, \&c.

\section{Sub-Genus STREPHOBASIS, Lea.}

P. Acad. Nat. Sci. Philad. 1861, type S. cornea, Lea.

Sub-genus of Angitrema, fide Tryon.

\section{Pleurocera [Strephobasis] olivaria.}

Strephobasis olivaria, Lea, Proc. Acad. Nat. Sci., 1862, Knoxville.

2. Alabama; ex c. Dr. T. Oldham.

\section{Genus GONIOBASIS, Lea.}

P. Acad. Nat. Sci. Philad. 1862, type [restr.] M. showalteri, Lea.

\section{Goniobasis alabamensis.}

Melania alabamensis, Lea, P. Acad. Nat. Sci., 1861.

5 Coosa Rv., Alabama; ex c. Rev. E. R. Beadle.

\section{Goniobasis boykiniana.}

Melania boykiniana, Lea, P. Philos. Soc., 1810,-" Columbus, Geo."

4. Georgia; ex c. Prof. W. Newcomb.

3 Alabama; ex c. Prof. W. Newcomb.

\section{Goniobasis pupoidea.}

Melania pupoidea, Anthony, Ann. Lyc., New York, 1854, Alabama. 6 Alabama; ex c. Rev. E. R. Beadle. 


\section{Goniobasis silicula.}

Melania silicula, Gould, P. Bost. S. II, 1847, Nisqually-Oregon.

\section{var. "shastaensis.}

Melania shastaensis, Lea, P. Acad. Nat. Sci. 1856, Shasta River, California, $\& c$.

3 California; coll. Prof. E. C. Stearns.

\section{Goniobasis circumlineata.}

Goniobasis circumlineata, Tryon, Am. J. Con. I, 1865, California.

5 California; coll. Prof. W. Newcomb.

\section{Goniobasis ellipsoides.}

Goniobasis ellinsoides, Lea, Obs. Unio, \&c., Tol. IX, for MLelania gracilior, P. Acad. Nat. Sci., 1861, Coosa River, Alabama [not MIel. gracilior of Authors].

4 Alabama; coll. Rev. E. R. Beadle.

The name of the species should stand as $G$ gracilior, Lea.

\section{Goniobasis coosaensis.}

Melania coosaensis, Lea, P. Acad. Nat. Sci. 1861, Coosa River.

4. Alabama; coll. Rev. E. R. Beadle.

\section{Goniobasis rubicunda.}

Melania rubicunda, Lea, P. Acad. Nat. Sci. 1861-Coosa River. 10 "Coosak" Kv., Alabama; coll. Rev. $\dot{\mathrm{E}}$. R. Beadle.

\section{Goniobasis draytoni.}

Goniobasis draytoni, Lea, P. Acud. Nat. Sci. 1862, Fort George, Oreyon.

4 California; ex c. Prof. W. Newcomb. 
10. Goniobasis nigrina.

Melania nigrina, Lea, P. Acad. Nat. Sci. 1856-Shasta county, California.

7 California; ex c. Prof. W. Newcomb.

\section{Goniobasis troostiana.}

Melania troostiana, Lea, Philos. Proc. II, 1841-Jefferson County, Tennessee.

1 Kentucky; ex c. Dr. F. Stoliczka.

12. Goniobasis aterina.

Goniobasis aterina, Lea, P. Acad. Nat. Sci. 1863-East Tennessee.

y Kentucky; coll. Rev. E. R. Beadle.

13. Goniobasis adusta.

Melania adusta, Anthony, P. Acad. Nat. Sci., 1860.

\section{var. cumberlandensis.}

Goniobasis cumberlandensis, Lea, P. Acad. Nat. Sci. 1860-Cumberland Gap, \&c., Tennessee.

7 Kentncky; ex c. Dr. F. Stoliczka.

\section{Goniobasis showalteri.}

Melania showalterii, Lea, P. Acad. Nat. Sci., 1861, Alabama.

4. Alabama; ex c. Prof. W. Newcomb.

\section{Goniobasis grata.}

Melania grata, Anthony, P. Acad. Nat. Sci., 1860.

5 Alabama; coll. Rev. E. R. Beadle.

\section{Goniobasis virginica.}

Buccinum virginicum, Gmelin, Syst. Nat., 1788.

6 Peunsylvania; coll. Rer. E. R. Beadle. 
They agree with Tryon's fig. 219.

5 Hudson Rv., New York; coll. Prof. W. Newcomb.

Near Tryon's fig. 287.

5 Silver Springs, Pennsylvania; ex c. Dr. F. Stoliczka.

var. multilineata.

Melania multilineata, Say, J. Acad. Nat. Sci., 1822.

3 New York; ex c. Prof. W. Newcomb.

A smaller form than Tryon's fig. 290, otherwise like it. Mr. Lea presented the Asiatic Society with some examples of the type form and also of this var.-see J. A. S. B. 1832, p. 77; as also some of $M$. conica, Say.

$$
\text { ? var. [? dist. sp.] }
$$

2 Pennsylvauia [? ]; ex c. Dr. F. Stoliczka.

\section{Goniobasis vanuxemiana.}

Melania vanuxemi.na, Lea, Trans. Amer. Philos. Soc., 1942-Alabama.

5 Alabama; coll. Rev. E. R. Beadle.

\section{Goniobasis solidula.}

Melania solidul, Lea, P. Acad. Nat. Sci., 1S61-Alabama.

4 Alabama; coll. Rev. E. R. Beadle.

$$
\text { ? var. [? dist. sp.] }
$$

2 Alabama; coll. Rev. E. R. Beadle.

Mixed wath the preceding. Ribbed at base.

\section{Goniobasis olivula.}

Melania olivula, Conrad, Amer. Journ. Sci., 1S34, Alabama

I Alabama; ex c. Prof, W. Newcomb. 
20. Goniobasis livescens.

Melania livescens, Menke, Syn. Méth. 1830.

12 Michigan; coll. Rev. E. R. Beadle.

5 New York; coll. Yrof. W. Newcomb.

var. cuspidata.

Melania cuspidata, Anthony, P. Bost. S., $18 \tilde{0}$.

2 Ohio; ex c. Prof. W. New comb.

\section{Goniobasis proxima.}

Melania proxima, Say, J. Acad. Nat. Sci., 1825.

7 N. Carolina; coll. Rev. E. R. Beadle.

\section{Goniobasis semicarinata.}

Melania semicarinata, Say, New Harm. Diss., 1819.

5 Ohio; ex c. Prof. W. Newcomb.

\section{var. varicosa.}

Melania varicosa (Ward), Haldeman, Mon. Limn. 1854.

2 Indiana; ex c. Prof. W. Newcomb [sent as "G. varicosa"].

var. exilis.

Melania exilis, Haldeman, Sup. Mon. Limn. $1 S 40$.

4. New York; ex c. Prof. W. Neweomb [as "G. exilis"].

$$
\text { ? var. [? dist. sp.] }
$$

6 Kentucky ; ex c. Rev. E. R. Beadle.

Mixed with $G$. aterina.

\section{Goniobasis nodulosa.}

Melania nolulosa, Lea, Philos. Proc. II, $18 \pm 1$-Tennessee.

2 Tennessee ; coll. Prof. W. Newcomb. 
24. Goniobasis decorata.

Melania decorata, Anthony, P. Acad. Nat. Sci., 1860.

6 Alabama; coll. Rev. E. R. Beadle.

\section{Goniobasis hydei.}

Melania hydeii, Conrad, New freshwater Shells, 1824.

3 Georgia; ex c. Prof. Newcomb.

\section{Goniobasis plicifera.}

Melania plicifera, Lea, Philos. Trans. VI, 1836-Wahlamat, near junction with the Columbia River.

1 Oregon [?]; ex c. A. Morelet, Esq.

A magnificent specimen of the rare typical form, as figured in the Amer. J. Conch. I, pl. XXIV, fig. 3.

var. oregonensis.

Melania plicifera, var. oregonensis, Tryon, Amer. J. Conch. I, 1864, pl. $X X I T$, fig. 4, Oregon.

10 Oregon; ex c. Prof. W. Newcomb, \&c.

var. bulimoides.

Melania plicifera, var. bulimoides, Tryon, l.c., fig. 5-6, Oregon.

8 Oregon; ex c. Prof. W. Newcomb.

27. Goniobasis carinifera.

Melania carinifera, Lamarck, Hist., 1822.

12 Georgia; ex c. Prof. Newcomb.

var. bella.

Melania bella, Conrad, New fresh̆water Shells, App., 1834.

4. Alabama; ex c. Prof. W. Newcomb.

\section{Goniobasis bulbosa.}

Melania bulbosa, Gould, P. Bost. S. II, 1847-Columbia River.

12 California; ex c. Profs. E. C. Stearns and W. Newcomb. 
They do not agree well with Tryon's figures.

29. Goniobasis acuta.

Melania acuta, Lea, Philos. Trans. IT, 1832-Tennessee River.

4. New York; ex c. Prof. W. Newcomb.

30. Goniobasis laeta.

Melania laeta, Say.

3 Alabama; ex c. Prof. W. Newcomb.

31. Goniobasis continens.

Goniobasis continens, Lea, P. Acad. Nat. Sci., 1862-North Alabama.

5 Alabama; ex c. Prof. W. Newcomb.

Sub-Genus SCHIZOSTOMA, Lea.

Amer. Phil. Soc. 1842, type M. excisa, and P. Acad. Nat. Sci. Philad. 1860 [not of Bronn, 1835, which=Euryomphalus]; =Schizochilus, Lea, Trans. Amer. Phil. Soc., $1852 ;=$ Gyrotoma, Shut. 1845.

Fide M. Brot, distinct Genus, as Gyrotoma, Shut., including Meseschiza, Lea.

32. Goniobasis [Schizostoma] babylonica.

Melania babylonica, Lea, Philos. Proc. IV, 1845-Ohio.

2 Alabama; ex c. Rev. E. R, Beadle.

Sub-Genus MESESCHIZA. Lea.

P. Acad. Nat. Sci. Philad., 1864, type M. grosvenorii, Lea.

Not represented in the Museum.

Genus ANCULOSA, Say.

J. Acad. Nat. Sci., 1821, type Mel. praerosa, Say; =Leptoxis, Brot and Auct. [as of Rafin. 1819].

\section{Anculosa costata.}

Anculotus costatus, Anthony, J. Bost. S. III, 1840, Cincinnati. 6 Olino; ex c. Dr. F. Stoliczka. 


\section{Anculosa patula [?]}

Anculosa patula, Anthony, P. Acad. Nat. Sci., 1860.

3 United States [juv. ? ]; ex c. Dr. F. Stoliczka [labelled as above].

This identification appears to me very doubtful.

\section{Anculosa squalida.}

Anculosa squalida, Lea, Philos. Proc. IV, 1845, Alabama.

2 Alabama; ex c. Prof. W. Newcomb.

\section{Anculosa picta.}

Anculosa picta, Conrad, Amer. Journ. Sci., 1834.

5 Alabama; coll. Rev. E. R. Beadle.

\section{Anculosa planospira.}

Anculosa planospira, Anthony, Ann. Lyc. New York, I'I, 1854.

1 United States; ex c. Dr. F. Stoliczka.

\section{Anculosa dilatata.}

Anculosa dilatata, Conrad, New freshwater Shells, App., 1854.

var. kirtlandiana.

Anculotus kirtlaudianus, Anthony, J. Bost. S. III, 1840, "Falls of the Kanawha."

6 Virginia; ex c. Prof. W. Newcomb.

\section{Anculosa subglobosa.}

Melania subglobosa, Say, J. Acad. Nat. Sci., $V, 1825$.

var. gibbosa.

Anculosa gibbosa, Lea, Philos. Proc. II, 1841-Tennessee.

10 Pennsylvania; coll. Rev. E. R. Beadle. 
var. tintinnabulata.

Anculosa tintinnabulum, Lea, Philos. Proc. IV, 1845, Tennessee and Alabama.

2 Alabama; ex c. Prof. W. Newcomb.

\section{Anculosa plicata.}

Anculosa plicata, Conrad, New freshwater Shells, 1834.

7 Tennessee; ex c. Prof. W. Newcomb.

1 Alabama; ex c. Prof. W. Newcomb.

\section{Anculosa tæniata.}

Anculosa tæniata, Conrad, New freshwater Shell.s, 1534

1 United States; ex c. Dr. F. Stoliczka.

$$
\text { ? var. [? dist. sp.] }
$$

3 United States; ex c. Dr. F. Stoliczka.

\section{Anculosa praerosa.}

Anculosa praerosa, Say, J. Acad. Nat. Sci., 1821.

5 Ohio; ex c. Prof, W. Newcomb.

\section{Anculosa dissimilis.}

Paludina dissimilis, Say, Nicholson's Enc., 1819.

10 Potomac Rv., \&c., Pennsylvania; coll. Rev. E. R. Beadle and Prof. W. Newcomb.

\section{Anculosa rubiginosa.}

Anculosa rubiginosa, Lea, Philos. Proc. II, 1841, Alabama.

10 Alabama; ex c. Prof. W. Newcomb.

13. Anculosa ampla.

Anculosa ampla, Anthony, Ann. Lyc. New York, VI, 1854.

2 Alabama; ex c. Prof. W. Newcomb. 
Sub-Genus EURYCAELON, Lea

P. Acad. Nat. Sci.1864, type Goniobasis umbonata; sub.genus of Goniobasis, fide Tryon.

\section{Anculosa [Eurycaelon] crassa.}

Anculosa crassa, Haldeman, Mon. Limn., 1841.

3 Tennessee; ex c. Prof. W. Newcomb.

\section{Anculosa [Furycaelon] anthonyi.}

Anculosa Anthonyi, Budd, Redfield, Ann. Lyc. New Forî, ГI, 1554.

1 Tennessee; ex c. Prof. W. Newcomb.

\section{Genus LITHASIA, Haldemun.}

Sup. Mon. Limniades, 1840, type Lith. obovata, Say.

\section{Lithasia obovata.}

Melania obovata, Say, New Harmony Diss. 1829.

4 Kentucky; ex c. Dr. T. OIdhain.

Sub-Genus $A N G I T K E M A$, Haldeman.

Sup. Mon. Limniades, Jan. 1841 [on cover], type Mel, armigera, Say.

\section{Lithasia [Angitrema] salebrosa.}

Melania salebrosa, Say, New freshwater Shells, 1854.

9 Tennessee; coll. Prof. W. Newcomb.

\section{Lithasia [Angitrema] geniculata.}

Litbasia geniculata, Haldeman, Sup. Mon. Limn. 1840; emend. Lex, as I. genicula.

1 Tennessee; coll. Prof. W. Newcomb. 


\section{Lithasia [Angitrema] jayana.}

Melania jayana, Lea, Philos. Proc. II., 1841, Tennessee; =M. robulina, Anthony, P. Bost. S. 1850.

2 Kentucky; ex c. Dr. T. Oldham

2 Tennessee; ex c. Prof. W. Newcomb [as "L. robulina, Anth."]

\section{Lithasia [Angitrema] armigera.}

Melania armigera, Say, J. Acad. Nat. Sci. 1821.

10 Texas and Indiana; coll. Prof. W. Newcomb and J. Le.ı, Esq. [As. Soc. Beng.].

\section{Lithasia [Angitrema] verrucosa.}

Pleurocera verrucosa, Rafinesque, Annals Nature, 19\%0; =Melania nupera, Say, New Harm. Diss., p. 260.

5 Kentucky; ex c. Dr. T. Oldham.

\section{Lithasia [Angitrema] subglobosa.}

Lithasia subglobosa, Lea, P. Acad. Nat. Sci. 1861, Tennessee. 4. Alabama ; ex c. Dr. T. Oldham.

Sub-family MELANIIN $\mathbb{E}$, H. \& A. Adams.

Genus MELANOPSIS, Férussac.

Essai d'une méth. conchil., p. 70, 1807; and Mém. Soc Hist. Nat. Paris, 1823.

\section{Melanopsis prærosa, Linnæus.}

Buccinnm præmorsum, Linnæus, Syst. Nat. $X, 1758$, Seville, emend. Ed.XII, as B. prerosum; = Buccina maroccana, Chemnitz [pars.]

18 Baalbeck and Rv. Litany [Colosyria]; coll. Dr. J. Auderson [27, $\left.11 \frac{3}{4}\right]$. 
Whorls shaped like Brot, pl. 45, fig 13, but spire more produced; a single specimen distinctly banded, like Bourg. Mal. Alg., pl. XVI, fig. 20.

12 Rv. Litany; coll. Dr. J. Anderson $\left[17 \frac{1}{2}, 8 \frac{3}{4}\right]$.

A shorter, more convex form, resembling Brot, pl. 45, fig. 1 ["M. buccinoidea"], but rather smaller; a single specimen is distinctly banded, exactly like Rossmássler, fig. 1889 ["M. wagneri, Parr."].

2 Rhodes; ex c. R. Hungerford, Esq.

50 Damascus; coll. Dr. J. A ndersou.

Close to Rossmássler, fig. 1885, and Brot, pl. 45, fig. 13.

4. Damascus; coll. Dr. J. Anderson [long. 24, diam. 10 mil.; sub. decollate].

Abnormal specimens with the spire cylindrically produced, much as in M. Brot's, pl. 45, fig. 7 ["Rhodes"].

30 Damascus; coll. Dr. J. Anderson $\left[16 \frac{1}{2}, 10\right]$.

Resembles Brot, pl. 45, figs. 3, 5 and 14; has a shorter spire than the preceding.

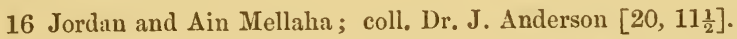

Resemble M. Brot's pl. 45, fig. 2, his M. bucrinoidea, Fér., a form distinguished by the more evenly rounded whorls.

20 Ain-es-Sultan [Elisha's Spring], Jericho ; coll. Dr. J. Anderson.

Resembles Rossmássler, fig. 1858, and Brot, pl. 45, fig. 9, \&c.

10 Ain Mellaha, Galillee; coll. Dr. J. Auderson.

7 Jericho; ex c. H. F. Blanford, Esq.

Typical specimens of the so-called $M$. buccinoidea.

30 Sahestan, W. of Shiraz; coll. W. T. Blauford, Esq.

Undistinguishable from the preceding.

1 Dead Sea; ex c. H. F. Blanford, Esq. [labelled "M. eremita, Trist."]

Does not seem to me to agree with Tristram's description. 
var. brevis.

Melanopsis prærosa, var. brevis (Parreyss), Mousson, Coq. Bellardi, 1854; fide Brot $=M$. ruinarum, Tarner, $m s$. from Baalbeck.

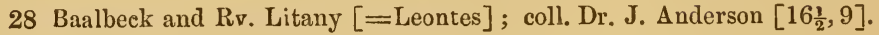

Even more convexly rounded than Brot's pl. 45, fig. 4, with the last whorl relatively unusually short; and lighter substance than usual, with callosity less developed.

var. variabilis.

Melanopsis variabilis (von dem Busch), Philippi, Abb.II, 1847, Schiraz and Persepolis.

Judging from M. Brot's figures, this variety is not $M$. mingrelica, Bayer, as I suspected from Issel's description; the latter's var. carinata must bear close affinity to my subvar. acuticarinata.

30 Persia; coll. W. T. Blanford, Esq. [24, 10].

From neighbourhood of Shiraz, I believe ; the original figures of Philippi are excellent, those of Brot very bad. These are what I consider typical specimens, resembling Philippi's fig. 8, "anfr. planiusculis."

12 Bandomir [Persia]; coll. W. T. Blanford, Esq.

20 Persia; coll. W. T. Blanford, Esq. $\left[21 \frac{1}{2}, 10\right]$.

Exactly resembling the preceding, but whorls more rounded, bearing much the same relationship to it as does $M$. buccinoidea to $M$. prerosa.

subvar. zonulata.

Philippi, l. c., fig. 10.

9 Persia; coll. W. T. Blanford, Esq.

subvar. nana.

15 Persia ; coll. W. T. Blanford, Esq. [16, 8].

2 Shiraz; ex c. A. Morelet, Esq. [as "M. fisciolata, Parr."] 
Show no trace of longitudinal ribbing. Long. 10, diam. $5 \frac{1}{2}$ mil.

\author{
subvar. bicolorata.
}

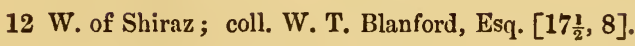

subvar. acuticarinata.

2 Persia; coll. W. T. Blanford, Esq. $\left[13 \frac{1}{2}, 7 \frac{1}{2}\right]$.

M. Bourguignat's pl. XV, fig. 25, Malac. Alger, gives a fair idea of this interesting form, which, however, is smaller, not decollate, of paler colour, obsoletely banded, with the keel even more developed.

subvar. decollata.

Philippi, l. c., fig. 7; ? M. fasciolata, Parreyss.

20 Persia ; coll. W. T. Blanford, Esq. [19, 9].

A very distinct, thin, eroded, slightly decollate variety, whorls usually less convex than in the quoted figure; under the lens minutely, flexuously striate; two or three show distinct, longitudinal, distant ribbing as well, much as in Rossm., fig. 1883 [M. tingitana, Morl.]. It is perhaps as closely allied to Issell's $M$. dorioe, - seems, indeed, to be a connecting link.

var. doriæ.

Melanopsis Doriæ, Issel, Moll. Persia, 1865, long. 24, diam.8 mil.-Kerman.

30 Kerman [S. Persia]; coll. W. T. Blanford, Esq.

From the same spot as Prof. Issel's type, with which they agree exactly.

subvar. carinifera.

10 Kerman; coll. W. T. Blanford, Esq.

A very interesting form, with a raised keel running along the suture. 


\section{Melanopsis maroccana.}

Buccina Maroccana, Chemnitz, Coneh.-Cab. I, 1795, Morocco, pl. 210, fig. 2080-1 $;=M$. Dufourii, Férussac, Mém. Soc. Paris, 1823, Valence. Almeria and Morocco $;=M$. hammanensis, Gassies, Act. Soc. Lin. Bord. 3 Ser., Vol. I [also of Mor. 1880].

1 Murcia; ex "Linnæa."

3 St. Denis du Sig [Oran]; coll. P. Joly, Esq.

Intermediate between M. Bourguignat's pl. XV, fig. 21 and fig. 23 [the latter "var. hammanensis, Graells."]

2 Morocco ; coll. W. T. Blanford, Esq.

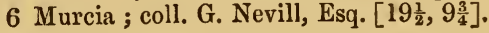

A solid, pale-coloured form, something like Bourguignat's pl. XV, figs. 17-18, but considerably smaller.

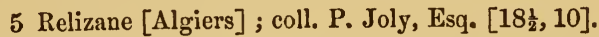

Intermediate between M. Bourguignat's figs. 20 and 23.

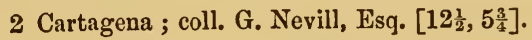

Somewhat resembling M. Brot's pl. 47, fig. 3, var. nana= M. etrusca, Villa, ms. ; it is exceedingly close to Rossmässler's original figure of $M$. lorcana, but is not excavate at the sutures.

18 Maison Carrée [Algiers] and Miliana; coll. P. Joly, Esq.

var. graellsi.

Melanopsis Graellsi, Villa [Disp. 1841 ?] ; Rossmässler, III, Icon. 1854, fig. 841.

1 Valencia; ex "Linnæa."

var. lorcana.

Melanopsis Lorcana, Guirao, Malak. Blät. 1854, Lorca.

2 Lorca [near Mnrcia]; ex "Linnæa."

var. saharica.

Melinopsis maroccana, var saharica, Bourguignat, Mal. Alg. 1864-long. 6 to 12, diam. 4 to 6 mil., Biskra, Ouargla, \&c.; fide Brot, var. of 11. prarosa, Linnaus.

6 Biskra; ex c. Colonel R. H. Beddome $\left[12,6 \frac{1}{2}\right]$. 
Seems to me intermediate between $M$. prcerosa and $M$. maroccana. The columella resembles that of the latter; but the shape of the last whorl and less expanded aperture (at base) show a closer affinity to the former species.

\section{Melanopsis cariosa.}

Murex cariosus, Linneus, Syst. Nat. 1758, Seville; fide Morelet $=M$. costellata, Fér. 1823 [pars], Seville and Morocco.

1 Guadalquivir ; ex "Linnæa."

\section{var. maresi.}

Melanopsis maresi, Bourguignat, Palcontol. Alg. 1862, S. of Prov. Oran [subfossil], long. 20 to '24, diam. 9 to 12 mil.; Bourg. in Malac. Alg. living specimens from Keriz, S. Prov. of Tunis; fide Paladilhe from Morocco.

2 Near Tlemeen [? subfossil] ; ex c. P. Joly, Esq.

Aperture less effuse at base, otherwise they agree well with M. Bourguignat's figures. M. Morelet, J. de C., 1880, p. 98, cousiders the form as distinct, and that $M$. maresi, Bourg. [1862], and his own $M$. tingitana [1864] ought to be united.

\section{Melanopsis costata.}

Melania costata, Otivier, Voy. Emp. Ottom. 1803, and M. costellata, Fér. 1823.

20 Ain Mellaha [Galilee]; coll. Dr. J. Anderson.

Near M. Brot's figure of the type form, pl. 46, fig. 4; also resembles Rossmässler, fig. 1904, but the ribbing is still more flexuous.

var.

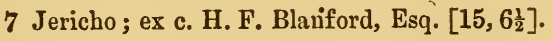

15 Ain-es-Sultan and Jordan; coll. Dr. J. Anderson.

Something like Rossm., fig. 1900-1, but smaller; I should have identified them as M. saulcyi, Bourg., but for their turreted spire. 
var. jordanica.

Melanopsis jordanica, Roth, Moll. Orient. 1839.

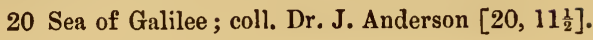

Agree with M. Brot's pl. 46, fig. 6.

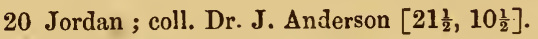

Agree with M. Brot's pl. 46, fig. 5, and Rossm., fig. 1905.

subvar. forticostata.

12 Lake Huleh [Palestine]; coll. Dr. J. Anderson.

A very remarkable, thick-ribbed form, with wonderfully produced spire; I can find no figure that represents it,long. 29 , diam. $11 \frac{1}{2}$ mil.

subvar. nana.

15 Sea of Galilee; coll. Dr. J. Anderson $\left[11 \frac{1}{2}, 7\right]$.

Like Rossm., fig. 1906, but smaller.

var.

3 Busrah, Beluchistan; coll. W. T. Blanford, Esq.

Unfortunately in very bad condition; it may prove to be an altogether new form. M. Brot's pl. 48, figs. 1 to 3 ["M. tingitana" $]$ are the nearest figures I can find; there is no basal canaliculation as in $M$. nodosa, Férussac.

Long. 15, diam. $7 \frac{1}{8} \mathrm{mil}$.

\section{Melanopsis kotschyi.}

Meianopsis kotschyi (v. d. Busch), Philippi, Abb.II, pl. 4, fig. 11, Persepo. lis.

30 Lake at Shiraz; coll. W. T. Blanford, Esq.

Evidently very variable; closely allied on the one hand to $M$. costata and on the other to $M$. prcerosa, especially to var. variabilis, subvar. fasciolata, Parreyss [or decollata, Nevill]. 


\section{Melanopsis trifasciata.}

Melanopsis trifasciata, Gray, Dieffenbach, Nov. Zel.II, $1843 ;=$ M. zelandica, Gould, P. Bost. S. 1847, New Zealand; =M. ovata, Dunker, Malak. Blät. 1861, Lake Rotoiti.

5 New Zealaud; ex c. Dr. F. Stoliczka.

\section{Melanopsis brevis.}

Melanopsis brevis, Morelet, Test. Nov. Austr. 1857, alt. 10-12, diam. 7 mil.

5 New Caledonia ; ex c. P. Bouvier, Esq.

\section{Melanopsis fragilis.}

Melanopsis fragilis, Gassies, J. de C. 1874, "Ouagap," long. 8.10, diam. 4-5 mil.

3 N. Caledonia; ex c. P. Bouvier, Esq.

\section{Melanopsis parreyssi.}

Melanopsis parreyssi (v. Mühlfeld), Philippi, Abb. II, pl. 4, fig. 15, Пun. gary.

20 Locality ?; ex c. Dr. F. St sliczka.

Agree with M. Brot's pl. 46, fig. 14, his "var. scalaris. Parreyss."

\section{Melanopsis brotiana.}

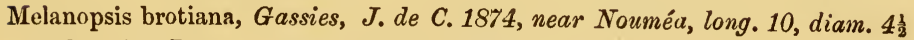
mil.; fide Brot? = M. carinata, Gassies, juv.

2 New Caledonia; coll. J. Caldwell, Esq.

\section{Melanopsis frustulum.}

Melanopsis frustulum, Morelet, Test. nov. Austral. 1857; fide Brot, =M. curta, Gassies, 1870, and vars. $=M$. variegata, Morelet, M. lentiginosa, Reeve, $M$. lirata, M. fasciata, M. fulgurans, and $M$. sordida, Gassies; vars. $=M$. fusca and souverbieana, Gassies.

\section{var. lineolata.}

Melanopsis lineolata, Gassies, J. de C., TI, 1857, Rv. Balade [not of Gray], emend. Gassies, Nouv. Caled. I, 1861, as M. livida.

5 New Caledunia; coll. J. Caldwell, Esq. 


\section{Melanopsis gassiesiana.}

Melanopsis gassiesiana, Crosse, J. de C., $X V, 1866, p .435, N$. Caledonia .

1 New Caledonia; ex c. P. Bouvier, Esq.

M. Brot questions if this species were not better united to Melanopsis brevis, Mor., as a variety. I am inclined to believe the same myself.

\section{Melanopsis fusiformis.}

Melanopsis fusiformis, Gassies, J. de C., 18\%0, N. Caledonia.

12 New Caledonia; coll. J. Caldwell, Esq.

Sub-Genus PSEUDHEMISINUS, nov.

Type Melanopsis esperi, Fér.

\section{Melanopsis [Pseudhemisinus] thermalis.}

Hemisinus thermalis, Brot, Mat. II, 1868 [as of Titius?], Hungary.

2 "East Europe"; ex c. Asiatic Soc. Bengal.

1 Taplocza, Hungary ; ex "Linnæa."

\section{Melanopsis [Pseudhemisinus] esperi.}

Melanopsis esperi, Férussac, Mém. Soc. Hist. Nat. Paris, 1823, River Laybach; from Plattensee, fide Brot.

2 Transylvania; ex c. Dr. T. Oldham.

3 Sava \& Agram, Croatia ; ex c. P. Joly, Esq., and ex "Linnæa."

\section{Melanopsis [Pseudhemisinus] acicularis.}

Melanopsis acicularis, Férussac, Mém. Soc. Hist. Nat. Paris, 1823, River Laybach, with var. cornea from the Danube.

2 Europe; ex c. Asiatic Soc. Bengal.

var. audebarti.

Melanopsis acicularis, var. Audebartii (Prévost), Férussac, l.c., from Töslau. 2 Agram, Croatia; ex c. P. Bouvier, Esq. 


\section{Genus HEMISINUS, Swainson.}

Treat. Malac. 1840, p. 341, type H. lineolatus, Gray.

\section{Hemisinus lineolatus.}

Strombus lineolatus, Gray, Wood's Ind. Test. Sup. 1828, Hab.?

2 Cuba; ex c. Prof. W. Newcomb.

M. Brot records as localities Jamaica, Venezuela, and Pernambuco, but not Cuba. The above resemble his pl. 38, fig, $6 \mathrm{~d}$.

\section{var. punctata.}

Hemisinus punctatus, Reeve, Icon. 1860, Pernambuco.

2 Brazil; ex c. Prof. W. Newcomb.

Like M. Brot's fig. 6 b, only smaller.

\section{Hemisinus ornatus.}

Melania ornatus, Poey, Mém. Cuba, 1852.

9 Cuba; ex c. Prof. W. Newcomb, \&c.

\section{Hemisinus brasiliensis.}

Melanopsis brasiliensis, Moricand, Mém. Soc. Phys. Geneva, IIII, 1839, Villa de Barra; fide Brot, var.=Mel. scalaris, Wagner.

2 Brazil; coll. A. Morelet, Esq.

$$
\text { var. elata, nov. }
$$

Conch.-Cab., II, pl. 40, fig. 12 A. as "var. B. elate turrita."

1 Brazil; ex c. Dr. F. Stoliczka.

\section{Hemisinus guayaquilensis.}

Melania guayaquilensis, Petit, Journ. de Conch., 1853, Guayaquil.

1 Guayaquil; ex c. A. Morelet, Esq.

\section{Hemisinus behni.}

Hemisinus behnii, Reeve, Icon. 1860, Pernambuco.

var.

2 Brazil ; ex c. A. Morelet, Esq.

1 Brazil; ex c. Prof. W. Newcomb. 
vàr.

1 Brazil ; ex c. Prof. W. Newcomb.

Resembles M. Brot's pl. 39, fig. 12 c.

Genus DORYSSA, H.\&A. Adams.

Gen. Moll. I, p. 304, 1856, type M. atra, Richard.

\section{Doryssa transversa [?].}

Melania transversa, Lea, Zool. 1850, Guiana.

var. subdevians, nov́. [? D. subdevians, n. sp.].

3 Amazons; ex c. Prof. W. Newcomb [labelled “Mel. transversa, Rv."].

Long. 26, diam. $12 \frac{1}{3}$ mil.

Strongly decollate, three or four whorls only remaining. Dark-brown colour, not light as M. Brot says Reeve's species always is. Longitudinal sculpture obsolete on the last whorl. But for its much larger size, M. Brot's pl. 35, fig. 10 A. [D. devians] well represents the form.

\section{Doryssa consolidata.}

Bulimus consolidatus, Bruguière, Enc. Méth., 1790 ; fide Brot $=H$. decollata,

Gmelin $=M e l$. circumsulcata, Busch, 1858=Mel. scarabus, Reeve.

3 “To cantines River," Brazil ; ex c. Prof. W. Newcomb [labelled “Mel. scarabus, Rv."].

Last whorl less convex than in M. Brot's figure; it is also less decollate, four whorls remaining.

\section{Doryssa aquatilis.}

Melania aquatilis, Reeve, Icon., 1859, Rio Branca, Guiana; fide Reeve [in "errata"] and Brot $=M e l$. branca, Rv., 1860, from Rio Branca.

1 South America; ex c. G. Nevill, Esq.

\section{Doryssa subimbricata.}

M elania subimbricata, Philippi, Abb. III, 1851, Hab.? [not Faunus subim. brcatus of Brot].

5 Brazil ; ex c. Prof. W. Newcomb [labelled "M. subimbricata, Ph.”] 
Agree exactly with Philippi's original figure, only a little smaller; they are, I believe, quite adult.

\section{Genus FAUNUS, de Montfort.}

Conch. Syst., 1810, type Strombus ater, Lin.; =Pirena, Lam., 1822, type

P. terebralis, Lam. $[=F$. ater $] ;=$ Melanamona, Bowditch.

Operculum resembling that of Tanalia; approximately onethird of the internal surface is covered with a black polished glaze; externally there is a somewhat indistinct striation.

\section{Faunus ater.}

Strombus ater, Linnœus, Syst. Nat., 1758, "in Asiœ paludibus" ;=Pirena terebralis, Lam., Moluccas; juv. =P. picta, Reeve, Ceylon; fide Brot= P. acus, Lesson, $N$. Ireland $=$ Melanopsis princeps, Lea, Cape of Good Hope=P. pagoda, Reeve, Habit.?-[spec. monstrosa]; var.?= P. cantori, Reeve, from Penang; fide Mousson from Java; fide Cum. ing, Philippines-fide Brot, Timor, Buru, Amboyna; var.?=P. nitida, v. d. Busch, from the Philippines.

2 Celebes [?]; ex c. Dr. F. Stoliczka [ex Landauer].

More slender and produced than my Ceylon specimens, with the last whorl not so broad; colour jet-black.

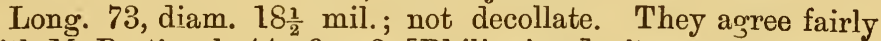
with M. Brot's pl, 44, fig. 3 [Philippines]; it seems to me to agree with Reeve's $F$. cantori, from Penang; size is of little importance.

1 Philippines [?]; coll. Baron F. von Richthofen.

A young specimen, in bad condition.

5 Ceylon; ex c. Dr. F. Stoliczka.

This I take to be the typical form; they agree with M. Brot's fig. 3 B. [var. picta, Rv.]. Long. 80, diam. 22 mil.

1 Nicobars [?]; ex c. Capt. R. C. Temple.

Closely resembling the preceding, only rather smaller. The locality is an important one, but requires confirmation.

2 Ceylon; coll. E. L. Layard, Esq. [ex c. Asiat. Soc. B.].

1 Habitat? ; ex c. Dr. F. Stoliczka. 
var. perdecollata, nov.

Brot, pl. 44, fig. 3, Habit.?

'10 Southern Province, Ceylon; coll. G. Nevill, Esq.

Long. $76 \frac{1}{2}$, diam. 21 mil. Apparently a form constantly characterized by the strong decollation, erosion of the surface, and iron-rust, reddish colour of the peristome, \&c.; mostly with specimens of an oyster attached, half as large as themselves. Very variable in size.

Sub-Genus MELANATRIA, Bowditch.

Elem. Conch., 1822, type Buc. fumineum, Gmelin.

\section{Faunus [Melanatria] flumineus.}

Buccinum flumineum, Gmelin, Syst. Nat.1788, Hab.? ; fide Brot, var. = Pirena aspera, Brot, var. = ? P. cecillei, Phillippi, 1849, Madcgascar, var. $=P$. plicata, Reeve = lamarcki, Val. $=$ fraterna, Lea $=M$ Mel. subimbricata, Phil. [spec.Juv.], var. = P. maura, Reeve, West Africa.

\section{var. plicata.}

Pirena plicata, Reeve, Icon., 1859, Madagascar.

1 Madagascar; coll. J. Caldwell, Esq.

Agrees exactly with M. Brot's pl. 43, fig. 2.

\section{Faunus [Melanatria] spinosus.}

Pirena spinosus, Lamarck, Anim. sans Vert., 1822, Madagascar.

5 Ranomafane [Madagascar]; coll. J. Caldwell, Esq.

I am informed that "Ranomafane" means "warm water," and that the above specimens were taken from some hot springs in the bed of the river; they agree with M. Brot's pl. 42, fig. $1 \mathrm{~A}$ and $\mathrm{B}$.

\section{? Genus PIRENOPSIS, Brot.}

Conch.-Cab. II, 1879, p. 408, type Melania costata, Quoy [Vanikoro].

Not represented in the Museum. 


\section{Genus CLAVIGER, Haldeman.}

Silliman's Journal, 1842, type Nerita aurita, Müller;=Vibex, Gray, P. Zool. S. 1847, type "Nerita aurita," not Vibex, Gray, 1840, or Oken, Zool. 1817 [fide Brot].

\section{Claviger auritus.}

Nerita aurita, Müller, Hist. Verm., 1774, Hab.? ; fide Brot, = Strombus tympanorum, Chemnitz, $=$ Io rota, Reeve, var. $=$ Mel. subaurita, Brot $=$ Mel. soriculata, Morelet.

1 Senegal; ex c. A. Morelet, Esq.

Agrees with M. Brot's pl. 36, fig. 7A.

var. subrota.

1 W. Africa; ex c. Asiatic Soc. Bengal.

Long. $34 \frac{1}{2}$, diam. 17 mil. A remarkable form, intermediate between M. Brot's pl. 36, fig. 7B, and pl. 37, fig. 2. Spire very short; nodules fewer than usual, more distant and more oblique, somewhat larger; white, with three brown bands on the last whorl.

\section{Genus LARTETIA, Bourguignat.}

Moll. Quartern. Paris, 1869, type L. belgarandi, Bourg. ; L. bourguignati, Pal. [only living sp.], from Peronne [Dep. Herault] ; fide Cles., Nachtr., $1878, p .126$, closely allied to Baicalia; fide Sdb., Conch. Vorwelt, p. $690=$ Goniochilus, Sdb. 1870, type Pleuroceras costulatum, Fuchs, fossil of the Lower Pleiocene from Radmanest = Micromelania, Bru. $\sin a, 1874$.

This and the following form are not in the Museum.

\section{Genus ONCOMELANIA, Gredler.}

Jahrb. 1881, p. 120, type O. hupensis, Gredl. [Prov. Hunan].

Genus TIPHOBIA, E. A. Smith.

P. Zool. S. 1880, type T. Horei, Smith [Lake Tanganyika]. 
Mr. Smith states in the P. Zool. S. for 1881, pages 277 and 293 , that the operculum is "melanoid," but that the absence of an epidermis in the species may necessitate the separation of the genus from the Melaniidoe.

Genus PYRGULA, Cristofori \& Jan.

Consp. meth. moll. Mantissa, 1832, type P. annulata, Jan; sect. Diana, Clessin, Malak. Blät. XXV, 1878, type P. thiesseana, Godet [not of Risso, 1826, gen. Piscium].

\section{Pyrgula annulata.}

Pyrgula annulata, Cristofori and Jan, Mantissa, 1832, Lombardy; var. =P. helvetica, Michelin, Guerin, Mag. Conch. 1831, Lake of Genevalong. 3, diam. 1 mil.

12 Lake Idro; ex c. P. Bouvier and C. F. Ancey, Esqrs.

8 Lake Garda, N. Italy; coll. Dr. H. Dohrn.

\section{Pyrgula thiesseana.}

Pyrgula thiesseana, Godet, ms., Kobelt, Jahrb. $T$, 1878, Missolunghi-long. 9 mil., and Rossm. Icon., fig. 1910 ; sect. Diana.

2 Lepanto, Greece; ex c. P. Bouvier, Esq.

Genus MOITESSIERIA, Bourguignat.

Monogr. Moitessieria, 1863, type Paludina simoniana, Charp., ms. [Toulouse].

This genus and the three next are still unrepresented in the Museum collection.

Genus LHOTELLIERIA, Bourguignat.

Bull. Soc. Toulouse, 187\%, type L. letourneuxi, Bourg. ; =Locardia, de Folin, J. de C., 1880, type L. apocrypha, de Folin [Rhône].

Genus PALADIIHIA, Bourguignat.

Monogr. Paladilhia, 1865, type P. pleurotoma, Bourg. [Montpellier]. 
Genus BUGESIA, Paladilhe.

Rev. Mag. Zool. 1866, type B. bourguignati, Pal. [Montpellier].

Von Martens, Stimpson, \&c., consider this genus, as also the four preceding groups, as belonging to the Rissoida, subfam. Hydrobiino.

\section{Genus MELANIA, Lamarck.}

Prod. 1799 and Syst. anim. sans Vert. 1801, type? Helix amarula, Lin. restr. H. and A. Adams, 1855 and Brot, 1874, type M. hastula, Lea.

I have followed Messrs. Brot and Adams in adopting the group of $M$. hastula as the typical section, although I consider that of $M$. amarula, Bolten's Genus Tiara, would have been more correctly adopted.

\section{Melania fuscata.}

Helix fuscata, Born, Test. Vindob. 1780, "Virginia" [not of the Con. Indica]; var. $=M$. macrospira, Morelet, Test. nov. Austral. 185\%, N. Caledonia.

I am unable after a long and careful comparison of numerous specimens of both, in all stages of growth, to separate the Fijian and Nicobarese forms. I can see no constant difference whatever, except the more conspicuously marginate suture, nor can I find any distinctive characters for the New Caledonian form; individuals vary in the slightly more or less convex shape of the whorls. It is very likely that Born's types of both $M$. fuscata and M. maculata came from the Nicobars.

4 Nicobars; coll. F. A. de Roepstorff, Esq.

From Nancowry, I believe; M. Brot's pl. 17, figs. 4a and 5, give a fair idea of them.

3 Nicobars; coll. Dr. F. Stoliczka.

Agree well with Born's original figure [=Brot's pl. 18, fig. 2]; also Brot's pl. 17, fig. 4 [M. aspirans], and one resembles his pl. 17, fig. 4. A. This I consider the young of the form.

1 Nicobars ; ex c. Capt. R. C. Temple.

Well represented by Brot's pl. 17, fig. 5 [M. macrospira]; long. 67 , diam. 16 mil. 
subvar. submaculata.

3 Great Nicobar ; coll. F. A. de Roepstorff, Esq.

Probably not adult; closely resemble Brot's pl. 17, fig. $4 \mathrm{D}$. [M. aspirans, var. picta].

5 Katchall ; coll. F. A. de Roepstorff, Esq.

Probably a more adult form of the preceding, well represented by Brot's pl. 16, fig. 7 A. [M. maculata, var.], which I expect is also a Nicobar specimen. There is a great tendency to deformity in this race; one of them is twisted like Eulima arcuata. Reeve's fig. 57 ["M. acutissima"-pars] also probably represents this form.

\section{var. aspirans.}

Melauia aspirans, Hinds, Ann. Mag. XIV, 1844, Fiji Islands, axis 23 lin.

5 Ovalau Fiji ; ex c. F. Layard, Esq., \&c.

Mr. Layard's two specimens were labelled " $M$. figurata, Hinds," a species from New Ireland, which also proves to be only a variety of this widely distributed species. Brot's pl. 17, fig. 4, though somewhat larger, well represents the above specimens. The variety, so far as I can see, is only really characterized by the more marked margination of the suture.

subvar. compacta.

4. Fiji ; coll. Dr. C. F. Tonnerre [A. S. B.].

A more attenuate, solid and contracted form, represented by

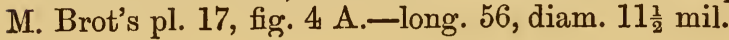

subvar, picta.

Melania picta, Hinds, Ann. Mag. XIV, 1844, New Ireland, axis 19 lin.

1 Fiji ; coll. Dr. C. F. Tonnerre [A. S. B.].

\section{Melania pantherina.}

Melania pantherina, von dem Busch, Malak. Blät. 1858, Philippines, and Reeve, Icon.fig. 38.

5 Guimaras and Cebu ; coll. R. Hnngerford, Esq. 


\section{Melania punctata.}

Melania punctata, Lamarck, Anim. sans Vert. 1822, Hab.? $=M$. albescens, Lea, P. Zool. S. 1850, Philippines, and Reeve, Icon., fig. 42.

2 Guimaras; coll. R. Hungerford, Esq.

5 Philippines [?]; coll. Baron F. vou Richthofen.

Agree well with M. Brot's pl. XX, fig. 4.

\section{var. monilis [?]}

? Melania monile, Mousson, J. de C. 185\%, Java, and Brot, Conch.-Cab. II, pl. 20, fig. \%, Moluccas.

6 Philippines [?]; coll. Baron F. von Richthofen.

\section{var. perdecollata.}

3 Philippines [?] ; coll. Baron F. von Richthofen. var. [? spec. juven. of var. monilis].

2 Philippines [?] ; coll. Baron F. von Richthofen. var. [? dist. sp.]

1 Philippines [?]; coll, Baron F. von Richtbofen.

\section{Melania clavus.}

Melania clavus, Lamarck, Hist. $V, 1822, H a b$. ? ; fide Brot $=M$. acuminata, Dkr., Phil. Alb., Habit. ? = M. acus, Lea, P. Zool. S. 1850, and Reeve, Icon. fig. 92, Guimaras = M. gaudiosa, Hinds, Ann. Mag. XIV, 1844, New Ireland ; var. $=M$. cristobalensis. Brot, Mat. II, 1868, S. Cris. tobal.

A very variable and widely distributed species; M. Brot's pl. 21, fig. 17, from an original and typical specimen of Lamarck's collection, may well be a young specimen of var. sobrius, Lea, from the Nicobars.

\section{var. sobrius.}

Melania sobrius, Lea, P. Zool. S. 1850, and Reeve, figs. 32 and 80, Siquijor.

20 Nancowry and Katchall; coll. Dr, F. Stoliczka, and F. A. de Roep. storff, Esq. 
One or two exactly resemble M. Brot's pl. 21, fig. 5-long. $31 \frac{1}{2}$, diam. 11 mil.; the others have the last whorl more contracted, evenly rounded, instead of slightly subangulate-long. $31_{2}^{1}$,

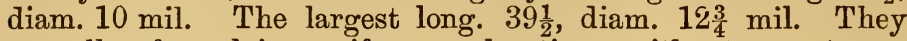
are all of a plain, uniform coloration, without any traces either of spots or bands.

subvar. cochlidium.

Melania cochlidium, Lea, P. Zool. S. 1850, Siquijor and Guimaras; Reeve, fig. $2 \%$.

3 Katchall ; coll. F. A. de Roepstorff, Esq.

The infra-sutural ledge is less marked than in the abovequoted figures, but still quite distinct, especially near the aperture.

\section{var. pirenoidea, nov.}

2 [Camorta? Nicobars]; coll.F. A. de Roepstorff, Esq.

A remarkable, "Pirena-like," strongly decollate and massive form, perhaps the origin of the observation in the voyage of the "Novara" of the finding of a species of Pirena at Katchall?

One specimen of 4 whorls measures long. 30, diam. 12 mil., and is well represented by M. Brot's pl. 21, fig. 16a, the apical whorls of course being left out of consideration; the other of 3 whorls, and the internal portion of a fourth has the last whorl more convex, with a slight infra-sutural shelf ; long. 30, diam. 13 mil. M. Brot's pl. 21, fig. 16, though smaller and with the last whorl more cylindrical, gives a very fair idea of it; both are of uniform coloration.

2 Camorta?; coll. F. A. de Roepstorff, Esq.

One is a quite young specimen of 7 whorls, slightly decollate, as in Brot's pl. 21, fig. 17 ; the other of medium growth, has 5 whorls remaining, none of which show the apical sculpture as does the preceding. I have little hesitation in referring both of them to this variety as non-adult forms.

subvar. perdecollata.

2 Nicobars; coll. F. A. de Roepstorff, Esq. 
A smaller form, still more decollate, only 2 to 3 whorls remaining. They are exactly represented by M. Brot's abovequoted pl. 21, fig. 16.

\section{var. plana.}

Melania plana, v. d. Busch, ms., Brot, Conch.-Cab. II, pl. 21, fig. 17a, as $?=M$. gaudiosa, Hinds.

4 Great Nicobar ; coll. F. A. de Roepstorff, Esq.

The only difference from the following two specimens seems to be that the apical sculpture is rather less developed.

2 Katchall [?] ; coll. F. A. de Roepstorff, Esq.

A very interesting form, fairly represented by the above figure, though the last whorl is relatively rather shorter still. The apical 8 whorls are very prominently and regularly longitudinally ribbed and spirally striated; last whorl, of course, smooth at base; of uniform dark olive-green coloration. I cannot help thinking they are from Great Nicobar like the preceding.

Anfr. 12 ; long. $28 \frac{1}{2}$, diam. $7 \frac{3}{4}$ mil.

\section{Melania crenulata.}

Melania crenulata, Deshayes, Lamarck, Hist. anim. s. vert. ed. 2, 1838, Hab. ?, for "Helix turrita crenulata," Chemnitz, Conch.-Cab. I, fig. 1230 .

2 Samar [Philippines]; coll. Prof. C. Semper.

One of these resembles Reeve's fig. 26, but is only about half the size; the other exactly resembles Ceylon specimens of var. confusa, and I cannot help thinking may have got mixed by some accident.

var. confusa.

Melania confusa, Dohrn, P. Zool. S. 1858, Ceylon, and Con. Indica, pl. 72, fig. 4, Ceylon.

I have never seen a specimen like M. Brot's pl. 14, fig. 9b.

6 Ceylon; coll. E. L. Layard [A. S. B.] and G. Nevill, Esqrs.

3 Ceylon; coll. E. L. Layard, Esq. [A. S. B.]. 
Perhaps the young form of the preceding. M. Brot's pl. 14, fig. 9c, gives a good idea of it, only the latter is a much larger form. Mousson's pl. XI, fig. 4, "M. porcata, Jonas," from Java, is also very close.

var. tirouri.

Melania tirouri, Férussac, Quoy and Gaim., pl.56, figs. 38-39, Deshayes, l. c., 1838, Hab. ?; fide Brot = M. lavis, Gray, Griff. Cuv. 1834 [not of Reeve].

4 Puniar River, Cuddalore; coll. Colonel R. H. Beddome.

1 Vizagapatam; ex c. Dr. F. Stoliczka.

Not represented in the Con. Indica; is near M. Brot's pl. 14, fig. 9a, but the spiral sulcations are more deeply incised. Long. 61, diam. 16 mil.

1 Cebu; ex c. R. Hungerford, Esq. [coll. Dr. Eastlake].

Resembles the preceding astonishingly.

1 Andamans; coll. F. A. de Roepstorff, Esq.

\section{Melania semicancellata.}

Melania semicancellata, v. d. Busch, Philippi, Abb. I, 1845, Java?

1 Sourabaya, Java; ex c. G. Nevill, Esq. len.

Agrees with type figure, only a little less ventricosely swolvar. [? distinct species].

1 Java?; coll. Baron von Richthofen.

Perhaps rather a variety of $M$. crenulata.

\section{Melania acutissima.}

Melania acutissima, v. d. Busch, Mal. Blät., 1858, Guadaloupe?; Reeve, Icon. figs. 57 and 58 ; Brot, Conch.-Cab., pl. 16, figs. 2 and $2 a$.

1 S. Cruz, Luzon; coll. R. Hungerford, Esq.

Long. $30 \frac{1}{2}$, diam. 9 mil, ; anfr. 9. 
Well represented by M. Brot's fig. 2 and Reeve's figs. 57 and 58 , but smaller and evidently not quite adult. It is important to be able to establish the true habitat of this species, as I am now able to do. The species resembles in many respects the form I have catalogued as $\boldsymbol{M}$. cerea? Reeve, in coloration, size, and the characteristic, forcibly reflected, thickened columella [exactly similar in both]; the apical spiral striation is, however, less distinct, but, above all, the whorls, instead of being unusually convex, are almost cylindrical, as in Reeve, fig. 57. The last whorl is distinctly subangulate, as mentioned by M. Brot in his invaluable work. The brown maculations resemble those of Reeve, fig. 57, though rather less distinct.

\section{var. perstriatula, nov.}

2 Guam; ex c. Prof. W. Neweomb [labelled "Mr. hastula"].

Wonderfully close to the preceding, but with more developed and regular spiral striation, only obsolete in the middle of the last two whorls; coloration uniform, last whorl subangulate, upper whorls more attenuately produced. Long. 30, diam. 8 mil.; anfr. 12.

\section{Melania plutonis.}

Melania plutonis, Hinds, Ann. Mag. XIV, 1844, Fiji ; Reeve, Icon. fig. 36 ; $?=M$. plicatilis, Mousson, J. de C., 1870 [juv.], and Brot, Conch.Cab., pl. 18, figs. 9 and $9 a$.

8 Navigators' Islands; coll. Dr. C. E. Tonnerre [A. S. B.].

\section{Melania ærea [?].}

Melania ærea, Reeve, Conch. Icon. 1859, fig. 61, Philippines; Brot, l. c., pl. 19, fig. 4a. [certe].

12 Luzon [?]; coll. Baron F. von Richthofen.

Long. 35, diam. $10 \frac{1}{2}$ mil. ; anfr. 10.

This can scarcely be the $M$. tristis of Reeve, as the upper whorls are spirally striate, and the columella is strongly callously incurved, even more so than in Mousson's pl. XI, fig. 5 [" $M$. aspirans"]; it differs from the latter by the more ventricose last whorl, the shorter and more convex spire, \&c.; the last whorl is smooth, scarcely striate even at base; slightly maculated with brown streaks in young specimens only. Brot's pl. 19, 
fig. 4a $[M$. cerea], gives a good idea of the shape of the form, only the columella is not sufficiently bent back. Reeve's original figure, however, seems to me scarcely the same species. Reeve's fig. 58 [M. acutissima] also closely resembles it.

\section{Melania subula.}

Melania subula, Lea, P. Zool. S. 1850, Panay; Reeve, Icon., fig. 62.

$$
\text { var. contracta, nov. }
$$

$3 \mathrm{Cebu}$; coll. R. Hungerford, Esq.

Anfr. 13-14; long. $36 \frac{1}{4}$, diam. 9 mil. Differ from the abovequoted figure in the more contracted whorls, the last one especially being less ventricose. Two are of a uniform darkviolet colour throughout; the third agrees exactly with the original description, and has the paler band next the suture, with short flames, \&c.

\section{Melania hastula.}

Melania hastula, Lea, P. Zool. S. 1850, Philippines and Conch.-Cab. II, pl. 16, figs. 3 and $3 b$; fide Brot $=$ Helix plicaria, Born $=$ Limax scutulatus, Martyn $=M$. costata, Auct. [not of Quoy] $=M$. flammulata, Busch $[$ not of Reeve] $=M$. picta, Rv. [not of Hinds] $=M$. acuta, $R v$. [not of Lea] $=M$. arroensis, $R v$.

M. Brot records as localities-Philippines, Fiji, Halmaheira, and Arrow Isl. Herr Brauer, S. B. Ak. Wien, LXXVII, 1878 , p. 184, states that Born's type of Mel. maculata is $M$. flammulata, Busch; and that Born's Mel. plicaria is M. costata, Quoy.

var. subacutissima, nov.

3 Katchall; coll. F. A. de Roepstorff, Esq.

I can find no figure to represent this well-characterized form amongst $M$. Brot's illustrations of $M$. hastula; it is nearest fig. 3 , but much smaller, with the apical ribbing more distinct.

Perhaps his pl.16, fig. 2a [M. acutissima] best represents it; indeed, I have felt tempted to refer it to that species, as it resembles remarkably closely the Luzon specimen I have already recorded, only it is a more solid shell, with distinct, though not prominent, longitudinal ribbing on the apical five whorls, instead 
of the spiral striation of $M$. acutissima. Spiral striation on base of last whorl distinct; upper whorls obscurely dotted and flamed with brown and peculiarly alternate, as in Brot's fig. 2 a. Anfr. 14; long. 36, diam. $9 \frac{3}{4}$ mil.

20 Andamans; coll. F. A. de Roepstorff, Esq.

Anfr. 7 ; long. 55, diam. 16 mil. Three adult, the others young.

subvar. subcrenulata, nov.

1 Andamans; coll. F. A. de Roepstorff, Esq.

Anfr. 4 ; long. 51, diam. $16 \frac{3}{4}$ mil.

var. subflammulata, nov.

1 Guimaras; coll. R. Hungerford, Esq.

Like Philippi's M. flammulata [Java], but with very prominent longitudinal ribs and distinct regular spiral striation, the latter a character which at once separates it from the preceding variety; coloration bright and distinct.

var. subcostata, nov. [? dist. sp.].

Reeve, fig. 29b, as M. costata, Quoy, Philippines.

6 Samar; coll. Prof. C. Semper [sent as "M. costata"].

I am by no means sure that these specimens really belong to the same species as the two preceding varieties, though evidently all these forms are united by M. Brot. The throughout prominent, slightly nodulose spiral keels are highly characteristic of this form, which is smaller than Reeve's above-quoted

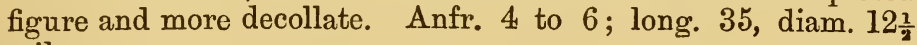
mil.

var. subpicta, nov.

Reeve, fig. 43, as M. picta [not of Hinds] ; Conch.-Cab. II, pl. 16, fig. 3 d., Halmaheira, "ex Landauer."

3 Ternate; ex Herr Landauer. 
var. pseudaculeus, nor.

1 Iles Marianes ; ex c. A. Morelet, Fsq. [labelled "M. aculeus ]."

This is certainly not the $M$. aculeus of Lea, in Brot or Hanley, Con. Misc., being much nearer the latter's fig. 32, 1. c., "M. fimbriata, Thorpe, ms.," of which, indeed, it might be a variety. The sutures are excavate, slightly swollen beneath, as in var. aspirans; the spire is rather less lanceolately produced than in any of the preceding forms ; though adult, it is scarcely decollate; coloration uniform brown; spiral striation very irregular on the lower whorls.

Anfr. 11 to 12 ; long. $51 \frac{1}{2}$, diam. $12 \frac{3}{4} \mathrm{mil}$.

\section{Melania zelebori.}

Melania zelebori, Brot, Mat. Mélaniens, III, 1872, Nicobars, long. 3\%, diam. $8 \mathrm{mil}$.

The young sometimes show obscure, short brown dashes [or streaks] below the suture and more or less throughout the last whorl.

10 Katchall, Great Nicobar, and Camorta ; coll. F. A. de Roepstorff, Esq.

Long. 53, diam. 16 mil.; anfr. 4.

var. nana, nov.

2 Great Nicobar; coll. F. A. de Roepstorff, Esq.

Strongly decollate, only 3 to 4 whorls remaining-long. $29 \frac{1}{2}$, diam. $10 \frac{1}{2} \mathrm{mil}$.

var. solidiuscula, nov.

6 Andamans; coll. F. A. de Roepstorff, Esq.

A very distinct variety, slightly decollate, 6 whorls-long. 37, diam. $11 \frac{1}{2}$ mil.; constantly distinguished from all the preceding by its greater solidity and by the less convex whorls, especially noticeable in the last one.

\section{Melania blatta.}

Melania blatta, Lea, P. Zool. S. 1850, Philippines.

1 Philippines?; coll. Baron F. von Richthofen. 


\section{Melania canalis.}

Melania canalis, Lea, P. Zool. S. 1850, Guimaras.

var. lanceolata, nov.

Brot, Conch.-Cab. II,"1878, pl. 19, fig. 11a, as "M. canalis, var."

7 Cebu ; coll. R. Hungerford, Esa.

No signs of any longitudinal ribbing even on the upper whorls, except on one specimen, so conspicuous a feature in Reeve's fig. 63.

var. [? distinct species].

4 Cebu ; coll. R. Hungerford, Esq.

Possibly the young of the preceding; if not, of some closely allied species. There is no trace of the broad band at the base of the aperture so characteristic of $M$. canalis.

Sub-Genus STRIATELLA, Brot.

Amer. Journ. Conch. VI, 1871; fide Brot, Conch.-Cab. II, type $M$. corporosa, Gould, and M. tuberculata, Müller.

15. Melania (Striatella) montrouzieri.

Melania montrouzieri, Gassies, Act. Soc. Lin. Bord., 1861, pl. V, fig. 10, N. Caledonia; fide Brot, var. M. mariei, Gassies, also perhaps= $M$. crepidinata, $R v$. [Java], and M. gracilina, Gld. [Tahiti].

It seems to me the New Caledonian form is scarcely separable from the Tahitian $M$. gracilina.

var. mageni.

Melania mageni, Gassies, l.c., pl. VI, fig. 10, N. Caledonia.

5 New Caledonia ; coll. J. Caldwell, Esq.

1 New Caledonia ; ex c. P. Bouvier, Esq. [as "M. sp. ? "].

Anfr. 3 ; long. 15, diam. $7 \frac{1}{2}$ mil. 
var. [? distinct $\mathrm{sp}$.$] .$

1 New Caledonia ; ex c. P. Bouvier, Esq. [as “ M. n. sp.?"].

Long. 25, diam. 9 mil. Possibly a large form of var. mariei, Gassies. M. Brot's pl. 24, fig. 7a, or fig. 14, gives an idea of the variety.

\section{Melania [Striatella] cylindracea.}

Melania cylindracea, Mousson, Moll., Java, 1849, "Pardana," Java.

2 Java ; ex c. A. Morelet, Esq.

\section{Melania [Striatella] arthuri.}

Melania arthurii,ן Brot, Amer. J. Conch. VI, 1871, for M. speciosa, Morl. 1857 [not of $\boldsymbol{A}$. Adams, 1853] = M. moreleti, Rv. 1860 [not of Deshayes ]; = M. exusta, Rv., fig. 74, Solomon Islands $=M$. gouldiana, Rv. fig. 115, N. Caledonia $=$ M. maurula, Gassies [not of Reeve, fide Brot $]=M$. jouani, Gassies.

I think there can be no question of the correctness of the synonymy of this species, as established by M. Brot.

3 New Caledonia ; ex c. P. Bouvier, Esq. [as " M. matheroni, Gass.]

4. New Caledonia; ex c. A. Morelet, Ksq.

var. pseudomaurula, nov.

1 New Caledonia; coll. J. Caldwell, Esq.

\section{Melania [Striatella] subexusta.}

Melania subexusta, Mousson, J. de C., 1869, Ovalau, vars. persulcata and albizonata; fide Brot $=M$. albizonata, Brot, 1871, and var. $=M$. ovalana, var. Kanathiana, Mousson, J. de C., 1870.

2 Ovalau [Fiji]; ex c. F. Lajard, Esq.

\section{Melania [Striatella] gracilina.}

Melania gracilina, Gould, P. Bost. S. 1859, Tahiti; fide Brot=M. incisa, Rv., fig. 118, Tahiti. 
var. ? [? dist. sp.].

2 Tahiti [?]; ex c. Dr. F. Stoliczka [labelled "M. incisa "]

A very doubtful form; I question the correctness of the recorded locality.

\section{Melania [Striatella] corporosa.}

Melania corporosa, Gould, P. Bost.S. 1847, Tahiti; fide Brot-long.40, diam. 16 mil.; and $=M$. bicolor, Brot, Rev.zool. 1860, Tahiti=M. ferrea, $\boldsymbol{R} v$. fig. 9, Borneo; and Grand Bassan=M. tahitensis, Dkr. 1866, Tahiti; var. $=M$. Iuteola, $D \mathrm{kr}$.

2 Tahiti; ex c. Mons. Morelet.

Well represented by M. Brot's pl. 24, fig. 7 .

$$
\text { var. Iuteola [?]. }
$$

Melania luteola, Dunker, Novara Reise, 1866,pl. 1, fig. 9, Tahiti-long. 21, diam. 7 mil.; fide Brot, M. luteola, Dkr. = M. unicolor, Tryon, 1865, and distinct species.

5 Tahiti; ex c. Dr. Stoliczka.

These do not agree with any figures in M. Brot's work, but are well represented by the above-quoted figure in the "Novara Reise;" they are only a little larger and with the columella more forcibly and regularly twisted. I cannot-separate this form as $M$. unicolor, as M. Brot's chief characteristic for the latter, the longitudinal ribbing on the upper whorls, is altogether wanting; near the apex, however, the spiral striation is minutely decussated, under the lens. Long. $30 \frac{1}{2}$, diam. 10 mil.; anfr. 8.

\section{Melania [Striatella] laxa.}

Melania laxa, Mousson, $J$. de C., 1869, Upolu, long. 16, diam. 8 mil., anfr. 2 to 3 ; fide Brot $=M$. cylindroides, Baird, Doy. Curacoa, Nieue or Savage Island; long. 9, lat. $4 \frac{1}{2}$ lignès.

6 Navigators' Islands; coll. Dr. C. E. Tonnerre [A. S. B.].

Anfr. $2 \frac{1}{2}$; long. $13 \frac{1}{2}$, diam. 7 mil. A very pretty and apparently constant form. 
22. Melania [Striatelia] scitula.

Melania scitula, Gould, D. Bost. S. 1847, Upolu, anfr. 5, "valde convexis."

var. subcylindrica, nov.

4. Navigators' Islands; ex c. Dr. F. Stoliczka.

In shape fairly resemble M. Brot's pl. 24, fig. 8 [M. unicolor, Pease], which is possibly scarcely specially separable; all four show traces on the upper whorls of indistinct longitudinal ribs; one of 4 whorls-long. $16 \frac{1}{2}$, diam. $6 \frac{1}{2}$, mil. -has the spiral striation of last two whorls altogether obsolete; two others have the last whorl smooth, and the fourth only is spirally striate, throughout. All of them are of uniform light-green colour, not banded.

23. Melania [Striatella] sublutosa, n. sp.

5 Gt. Nicobar ; coll. F. A. de Roepstorff, Esq.

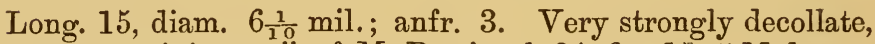
an exact "miniature" of M. Brot's pl. 24, fig. 15, "M. lutosa, Gould," from Upolu. Unfortunately I have not come across a young specimen, so as to be able to examine the sculpture of the upper whorls, if there be any.

\section{Melania [Striatella] turriculus.}

Melania turriculus, Lea, P. Zool. S. 1850, Luzon; = M. perpinguis, Reeve [not of Hinds].

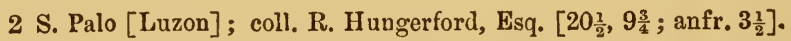

Well represented by M. Brot's fig. 7A and Reeve's fig. 113B.

1 Locality? ; ex c. G. Nevill, Esq.

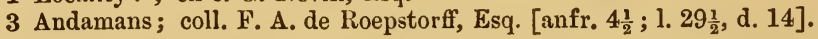

One is a magnificent adult specimen, well represented by Reeve's fig. 113A; the other two are young, with the spire nearly perfect and distinct ribbed longitudinally near the apex; all of them have the characteristic brown "flecks" below the suture.

var. nov. [? dist. sp.].

5 Magajay and S. Palo [Luzon]; coll. R. Hungerford, Esq. 
Dr. Hungerford sent these specimens mixed with the preceding. I think, however, they must be distinguished, as they are of a uniform sombre coloration, without any sculpture whatever; they are of all stages of growth, but still are in all cases decollated and eroded; M. Brot's pl. 24, fig. 3A. [" $M$. kanaiensis"],'fig. 7 ["M. corporosa"], and fig. 15 [M. lutosa"], well represent the form. Long. 37, diam. 15 mil.; anfr. 3.

\section{Melania [Striatella] graeffei."}

Melauia graeffei [Mousson], Brot, Conch.-Cab. II, 1877, Fiji, and of Cat. Mus. Godeffroy; $=$ M. lutosa, Gld., var., Mousson, J. de C., 1869.

1 Ovalau ; ex c. F. Layard, Esq. [labelled "M. costata"].

\section{Melania [Striatella] newcombi.}

Melania newcombi, Lea, P. Acad. Philad., 1S56, Oahu; = M. oahuensis, Brot $[$ as of Pease]; var. = M. contigua, Pease, Am. J. Con.1870, Kanai,-long. 28, diam. 9 mil.

6 Sandwich Islands; coll. H. Pease, Esq., and Prof. W. Newcomb.

\section{Melania [Striatella] unifasciata.}

Melania unifasciata, Mousson, Moll. Java, 1849.

20 Java; coll. Baron F. von Richthofen.

Very variable, as regards sculpture; some have distinct longitudinal sculpture near the apex, which in others is almost or altogether obsolete; there are 1 to 3 spiral, incised striæ on the lower portion of each of the last few whorls. The basal band is less distinctly marked than in Mousson's figure,-indeed, generally not visible at all within the aperture. A single specimen is of a uniform dark-violet colour; several are slightly maculated with brown; these resemble very closely $M$. tigrina, Hutton.

var. [? dist. sp.].

2 Badulla, Ceylon; coll. F. Layard, Esq.

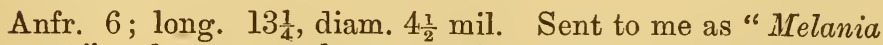
n. sp. ?" ; almost smooth, traces of sculpture only being here and there discernible; basal band round the columella very broad and distinct, otherwise of a purplish-brown uniform coloration. 
28. Melania [Striatella] recta.

Melania recta, Lea, P. Zool. S. 1850, Siquijor: and Negros-long. 1. 7, diam. $0 \cdot 5$ poll.

3 Cebu; coll, R. Hungerford, Esq. [36, 12].

\section{Melania [Striatella] nevilli.}

Melania nevillei, Brot, Conch.-Cab. II, 1874, pl. 22, fig. 13-long. 38, diam. 11 mil, anfr. 6.7; for M. andamanica, G. Nevill, ms. "in coll." Andamans.

6 Camorta [?]; coll. F. A. de Roepștorff, Esq.

A close ally of $M$. nicobarica, constantly, however, distinguished by the almost cylindrical instead of more or less convex whorls, the upper ones not abruptly attenuate, and the last one not tumidly swollen; the sculpture is very similar, but the markedly more strongly incised spiral striation at the base whorls appears to be characteristic.

var. andamanica, nov. [vel M. andamanica, n. sp. ?].

Possibly a distinct species, though at present, I believe, it is correctly classed as a local variety of $M$. nevilli. It is at once distinguished by the upper three or four whorls being invariably longitudinally ribbed, the ribs being not at all prominent and slightly but distinctly arcuate; quite young specimens show this ribbing throughout, the ribs only becoming obsolete on the lower portion of the last whorl. Generally but slightly decollate, eight whorls remaining; covered with a rust-brown coating, beneath which the shell is light green, irregularly and very slightly spotted with brown below the suture; throughout spirally striated; a single specimen only has this spiral sculpture obsolete on the central portion of the last two to three whorls. The majority of specimens are smaller than type figure of $M$. nevilli, and have the last two whorls increasing much more rapidly in breadth, in this more resembling M. Brot's pl. 22, figure 8B; the last whorl is, however, never convexly swollen as in $M$. nicobarica.

20 Andamans; coll. Colonel Ford, Dr. J. Anderson, \&c.

subvar. semilævigata, nov.

1 Andamans; coll. Colonel Ford. 
subvar. appressa, nov.

8 Andamans; coll. Dr. J. Anderson, \&c.

The last two whorls are contracted much as in typical $M$. nevilli.

\section{Melania [Striatella] nicobarica.}

Melania nicobarica [Mörch], Reeve, Con. Icon. 1859, Nicobars.

20 Katchall, \&c., Nicobars; coll. F. A. de Roepstorff, Esq., and Dr. F. Stoliczka.

22 S. Audamaus; coll. F. A. de Roepstorff, Esq., and Dr. F. Stoliczka.

subvar. canaliculata, nov.

The infra-sutural shelf is sometimes as sharply defined as in Reeve's figure of $M$. cochlidium; one specimen, indeed, has it developed on the three last whorls.

10 Nicobars; coll. F. A. de Roepstorff, Esq.

13 S. Andaman; coll. F. A. de Roepstorff, Esq.

subvar. gigantea.

Anfr. 4; long. 43, diam. 17 mil.

1 Camorta; coll. F. A. de Roepstorff, Esq.

10 S. Andaman; coll. F. A. de Roepstorff, Esq.

var. fusiformis, nov.

2 S. Andaman; coll. F. A. de Roepstorff, Esq.

Well distinguished by its produced and fusiform "shape; the whorls increase regularly and are more convex than those of the type form, in which the upper whorls are abruptly and contractedly attenuate, the lower ones increasing rapidly in size, especially the last, which is always very tumid. In var. fusiformis, on the contrary, it is only a trifle more swollen than the preceding one. More solid and of brighter coloration; sculpture rather less distinct.

Anfr. 9 ; long. $32 \frac{1}{2}$, diam. 9 mil.

var. [? dist. species].

6 "Nicobars" [?]; coll, Dr, F. Stoliczka, 
This locality requires confirmation. I believe it to be altogether distinct from Mel. nicobarica, but place it here temporarily until the exact locality is authenticated. It is of a light green colour, obscurely spotted with brown below the suture; whorls much more convexly rounded, columella being correspondingly twisted back; the apical whorls are less numerous and less attenuate, spiral striation less regularly and deeply incised, altogether or almost obsolete on the upper portions of the last few whorls; under a lens the first few are minutely decussated in the interstices. It appears also to be closely allied to a form found by Baron v. Richthofen, which I have recorded as $M$. cerea, Rv. var.

var. perstriatula, nov.

10 Andamans [?]; ex c. Dr. F. Stoliczka.

The last two whorls are throughout regularly and evenly, finely spirally striated.

\section{Melania [Striatella] crebra.}

Melania crebra, Reeve, Con. Icon., 1860, fig. 162, Guimaras; var. from Nicobars, Brot, pl. 33, fig. 2, as of Sect. Tarebia.

12 Preparis Island [Bay of Bengal] ; coll. Dr. F. Stoliczka, and J. WoodMason, Esq.

I think it possible M. Brot's pl. 27, fig. 1A may represent one of these specimens. Dr. Stoliczka sent some specimens to the dealer Landauer. Long. 35, diam. $11 \frac{3}{4}$ mil.

1 S. Andaman; coll. F. A. de Roepstorfe, Esq.

30 Nancowri and Katchall [Nicobars]; coll. F. A. de Roepstorff, Esq.

A smaller form, the largest-long. $31 \frac{1}{4}$, diam. $10 \frac{1}{2}$, in shape well represented by pl. 27 , figs. 2 and $2 \mathrm{~A}$, 1. c., the $M$. zengana, Mor., from Zanzibar. Specimens are often curiously contorted, like some species of Eulima and as well shown in the above fig. 2A. The sculpture is much more prominently developed, more uniform, otherwise not unlike that of fig. 1A, l. c. Brot's pl. 33, fig. 2, evidently represents one of this form, although the sculpture of the last whorl is more conspicuous than in my specimens. I consider this species as very closely allied to M. tuberculata.

1 South Andaman [?] ; coll. F. A. de Roepstorff, Esq.

Long. $29 \frac{1}{2}$, diam. $9 \frac{3}{4} \mathrm{mil}$. 
subvar. emaciata.

12 Katchall and Nancowri; coll. F. A. de Roepstorff, Esq.

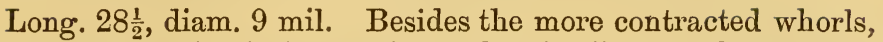
the coloration is darker and the longitudinal sculpture less developed.

\section{Melania [Striatella] tuberculata.}

Nerita tuberculata, Müller, Hist. Verm. 1774, Coromandel; var. $=M$. rodericensis, Smith, Ann. Mag. XVII, 1876, Rodriguez, long. 16, äiam. 5, and var. major, $l .23, d .7$ mil.

M. Brot seems to imply, l. c., page 250, that the Nile form is the typical one; this is erroneous. The exceedingly common Madras form is clearly pointed out by Müller. It does not appear to be figured by M. Brot; the Con. Indica, pl. 75, figs. 1 and 4, give a good idea of it. M. Brot is altogether mistaken in his views as to $M$. tigrina, Hutton, and $M$. pyramis, Benson, which are perfectly correctly identified and excellently figured by Mr. Hanley on plate 110 of the Con. Indica.

6 Pulla River, near Cuddapa; ex c. Madras Museum.

12 Cuddapa [Madras Pres.]; coll. Dr. F. Stoliczka.

2 Poona; coll. Dr, F. Stoliczka.

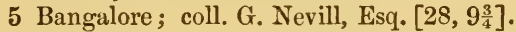

50 Madras; coll. Dr. F. Stoliczka, H. F. Blanford and G. Nevill, Esqrs.

Sometimes with, but oftener without, a broad brown band, quite at the base of the last whorl; occasionally without any brown markings whatever.

5 "Laggalle" [ubi ?]; ex c. Dr. F. Stoliczka.

12 "Borneo" [??]; ex c. Madras Museum.

50 Calcutta; coll. G. Nevill and J. B. Baxter, Esqrs.

1 Bombay; ex c. Dr. F. Stoliczka.

20 Rajputana; coll. C. A. Hacket, Esq.

A lanceolate, compressed, almost colourless form, with much developed spiral sculpture.

11 Derbund and Kutch; coll. Dr. F. Stoliczka.

Something like the preceding, but more richly coloured.

12 Mahé, Seychelles; coll. G. Nevill, Esq.

A rather small, not decollated, form which it is impossible to separate from Indian specimens even as a sub-variety. 
Long. 22, diam. 7 mil.

7 Mauritius; coll. G. Nevill [24, 9] and J. Caldwell, Esqrs.

2 Rodriguez; coll. J. Caldwell, Esq.

Doubtless the insufficiently described $M$. rodericensis of Smith.

3 Poona; coll. Rev. J. B. Fairbank [36, 111 1 .

6 "Calcutta" [?]; ex c. Asiatic Soc., Bengal [anfr. 5 ; 34, 12].

A giant, strongly decollated form; more or less subgranulose, especially discernible in the young.

20 Chandernagore; coll. Colonel G. B. Mainwaring.

This is the form I take to be the $M$. pyramis of Benson, as also are the two following.

4. Kathiawar; coll. F. Fedden, Esq.

8 Locality?; coll. Dr. F. Stoliczka.

3 Colombo; coll. G. Nevill, Esq.

A form with an acutely produced spire and contracted, short, convex whorls, very different from var. layardi. It is of course not the young form of the latter, which I know perfectly well.

8 Chandernagore and Rajmahal; coll. Colonel G. B. Mainwaring.

20 Raniganj; coll. Dr. F. Stoliczka.

14. Barak River, Cachar and Roorkee; coll. J. Wood-Mason, Esq.

Undoubtedly true typical Melania pyramis of Benson, as correctly figured in the Con. Indica, pl. 110, fig. 3; the Mel. pyramis of Brot is quite different.

4 Bimlipatam [?]; coll. J. Wood-Mason, Esq.

The typical form of Müller.

6 Locality ?; ex c. Asiatic Soc., Bengal

Very possibly, original typical specimens of $M$. pyrarnis from Mr. Benson?

Long. 34, diam. $12 \frac{3}{4}$ mil. ; anfr. 5 .

4 Locality ?; ex c. Asiatic Soc., Bengal.

Exactly represented by Con. Indica, pl. 120, fig. 3, probably typical specimens from either Hutton or Benson. 
Long. $23 \frac{3}{4}$, diam. 8 mil.

3 Ceylon; coll. E. L. Layard, Esq. [A. S. B.].

var. subcanaliculata, nov. [? dist. sp.].

3 Silhouette, Seychelles; coll. G. Nevill, Esq.

The nearest figure I know in M. Brot's work is pl. 27, fig. 1 ["M. pyramis "]. It also agrees fairly with his description, but cannot, I think, be Benson's Indian form, which is undoubtedly the one figured in the Con. Indica.

The last whorl is slightly subangulate, instead of convex, the apical longitudinal sculpture distinct, coloration sombre, suture slightly canaliculate.

Long. 29, diam. 9 mil.; anfr. 7.

var. canaliculata, nov. [? dist. sp.].

7 Locality ?; ex c. Dr. F. Stoliczka.

Unfortunately, there was no label with these most interesting specimens. Resembling the preceding, but the spire more produced, the whorls more planulate, the last whorl more compressed, longitudinal ribbing more developed, coloration brighter.

Long. $28 \frac{1}{2}$, diam. $8 \frac{1}{4}$ mil.; anfr. 8 .

var.

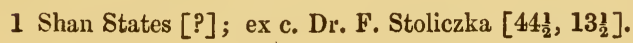

var. plicifera.

Mousson, Moll.Java, 1849, p. 73, pl, XI, fig. 7.

4 Java; coll. Baron F. von Richthofen.

A smaller form than the type figure, with less produced, shorter, and more convex whorls, bearing some resemblance to var. pyramis.

var. subcrebra, nov.

2 Bandarewella, Ceylon; coll. F. Layard, Esq. 
Sent to me as " $M$. tuberculata, var." It is a very interesting subgranulose variety, approaching $M$. crebra, Lea; of a uniform straw coluur; spire not much produced; last whorl convex.

Long. 22, diam. $7 \frac{3}{4}$ mil. ; anfr. 6-7.

\section{var. layardi.}

Melania layardi, Dohrn, P. Zool. S. 1858, Ceylon.

5 Ceylon; coll. E. L. Layard, Esq. [A. S. B.].

16 Colombo and Balapiti; coll. G. Nevill, Esq.

16 Kandy; coll. G. Nevill, Esq.

Fine specimens of the typical form of M. layardi.

12 Kandy; coll. G. Nevill, Esq.

Living with the preceding, separable by the more or less well developed, longitudinal sculpture; the whorls seem as a rule more convexly swollen. Can this be a sexual difference?

1 Kandy; coll. G. Nevill, Esq.

A remarkable, attenuate and contracted, "depauperate" specimen. Longitudinal ribbing distinct.

5 Kandy; coll. H. F. Blanford, Esq.

5 S. Canara; coll. Colonel R. H. Beddome.

The first specimens, I believe, of this very characteristic variety described from Continental India. They are a very fine form-long. 33, diam. 10 $\frac{3}{4}$ mil. -7 whorls.

var. tamsii.

Melania tamsii, Dohrn, Zeits. Mal., 1845, S. Antao.

6 S. Antaô and S. Nicolau [Cape de Verde Islands]; coll. Dr. H. Dohrn.

var. tigrina [? dist. sp.].

Melania tigrina, Hutton, J. A. S. B., 1849, Afghanistan, and Pinjore below Simla, \&c., Con. Indica, pl. 110, figs. 1-2 [bene].

Quetta [Beluchistan] ; coll. Captain F. W. Hutton [A. S. B.]. 
Typical, original specimens of $M$. tigrina. Remarkable for the canaliculate suture and convexly tumid last whorl; very solid, strongly truncated, \&c.

15 Mandi; coll. A. G. Young, Esq.

12 Mandi; ex c. Dr. F. Stoliczka.

Well represented by the Con. Indica, pl. 110, fig. 2; several specimens show a tendency to canaliculation of the suture.

subvar. [?].

6 Salt Range, Rajputana; coll. C. A. Hacket, Esq.

Long. $36 \frac{1}{2}$, diam. $11 \frac{1}{2}$ mil. ; anfr. 9 . A fine, solid form, with peculiarly impressed suture ; regularly, though somewhat superficially, spirally striated, apical whorls only subobsoletely, longitudinally striated; light green, mottled with brown maculations.

var. [? dist. sp.].

?Brot. Conch.-Cab. II, pl. 26, fig. $4 A$ [not fig. 4], as M. adspersa, Troschel.

1 Moulmein ; coll. Dr. F. Stoliczka.

A very remarkable form and brightly coloured, the last whorl shortly subangulate, aperture everted; spirally striate, without longitudinal sculpture, columella perpendicular, abruptly angled at base; anfr. $6 \frac{1}{2}$; long. $26 \frac{1}{2}$, diam. $10 \frac{1}{10}$ mil.

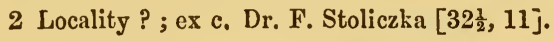

Probably from Burma. None of these agree with the Shan States form figured in the Con. Indica, pl. 110, fig. 4.

\section{var. orissaënsis, nov.}

1 Cuttack, Orissa ; coll. J. Caldwell, Esq̀.

A remarkable form; spire short, with ventricose whorls, substance thick, coloration uniform green (or almost so), longitudinal ribbing remarkably developed, even on the last whorl.

Long. 25, diam. 9 [vix] mil. 
var. cochinchinensis, nov.

1 Cochin China ; ex c. P. Bouvier, Esq.

Long. 34, diam. 11 mil.; very like the preceding, spire more produced, and longitudinal sculpture obsolete on the last whorl. It is not the M. pyramis, Phil., or $M$. gemmulata, Rv. Possibly, it may be the species recorded under that name by Morelet, Ser. Conch. IV.

var. chinensis, nov.

3 Swatow ; coll. R. Hungerford, Esq.

Long. $2 \dot{5}$, diam. 10 mil. ; anfr.' 5.

Some resemblance to M. Brot's pl. 14, fig. 1, his M. hanleyi from "India"; it differs from the following subvariety by its more everted aperture and by the columella not being bent back.

4 Yomati, opposite Hongkong; coll. R. Hungerford, Esq.

Anfr. 5 ; long. 28, diam. $9 \frac{3}{4}$. Fairly represented by M. Brot's pl. 26, fig. $11 \mathrm{~F}$, from "Mauritius and Madagascar." The only difference I can see is the spirally twisted columella and somewhat brighter coloration.

8 Amoy; coll. R. Hungerford, Esq.

With the longitudinal sculpture more or less obsolete.

$$
\text { var. dembeana. }
$$

Melania dembea, Reeve, Con. Icon., 1860, fig.161, Lake Dembea [Abyssinia].

14 "Aimlat, Samhar," Abyssinia ; coll. W. T. Blanford, Esq.

\section{subvar. luteomarginata.}

30 Beluchistan; coll. W. T. Blanford, Esq.

Long. $37 \frac{1}{2}$, diam. 13 mil.

2 Kalagan; coll. Museum Collector.

subvar. flavida, nov.

60 Pishin, \&c., Beluchistan; coll. W. T. Blanford, Esq.

16 Kalagan and Karman, S. E. Persia ; coll. Museum Collector. 
var. myadoungensis, nov.

20 Myadoung, Upper Burma ; coll. Dr. J. Anderson.

A very distinct form, remarkable for its " terebra-like " produced spire, its contracted or appressed whorls (especially the last); the spiral, undulating sculpture is more acutely prominent than in any form I know; all the whorls, except the last, have a light longitudinal ribbing as well; of a light yellowish-green colour, prettily marbled with brown. Anfr. 8 ; long. $27 \frac{1}{2}$, diam. $7 \frac{9}{10}$ mil.

ITo figure in M. Brot's work gives an idea of it-pl. 26, fig. $11 \mathrm{~A}$, being the nearest; pl. 74, fig. 1 of the Con. Indica resembles it, only the last whorl is too ventricose, \&c.

subvar. subplicifera.

7 Myadoung; coll. Dr. J. Anderson.

A form nearer Reeve's fig. 109B.; distinguished from the preceding by the more developed longitudinal sculpture throughout.

var. fasciolata.

Melania fasciolata, Olivier, Voy. Emp. Oth., 1807, pl. 31, fig. 7, Alexandrie.

20 "Highest point of the Desert, at Helouan, 15 miles south of Cairo;" coll. Dr. J. Anderson.

Bleached, with a subfossil appearance.

Long. 24, diam. 7 mil.

3 Constantine ; ex c. Colonel Beddome.

3 Biskra ; coll. P. Joly, Esq.

subvar. gigantea, nov.

4. Ouargla [Desert south of Algiers]; coll. P. Joly, Esq.

Decollate-anfr. 8 ; long. 41, diam. 14 mil.

Bleached, with a subfossil appearance.

var. judaica.

Melania judaica, Roth, Malac. Blät., 1855, Dead Sea.

20 Sea of Galilee; coll. Dr. J. Auderson. 
var. pseudo-truncatula, nov.

Brot, Conch..Cab., II, 1874, pl. 26, fig. 11F.

4 Mauritius ; coll. J. Caldwell, Esq.

12 Bourbon; coll. G. Nevill, Esq.

Resemble fairly M. Brot's above-quoted figure; rather smaller.

1 Locality ?; ex c. Dr. F. Stoliczka.

A remarkable form, perhaps distinct.

var. appressa, nov.

2 Mauritius; coll. J. Caldwell, Esq.

Much contracted, spire elongately drawn out, columella characteristically twisted backwards, very slightly decollate; long. $29 \frac{1}{2}$, diam. 9 mil.

No longitudinal sculpture, the spiral keels acute.

\section{Melania [Striatella] gemmulata.}

Melania gemmulata, Reeve, Icon., 1859, fig. 86, Hab.?

2 West Indies?; ex c. G. Nevill, Esq.

\section{Melania [Striatell.a] fontinalis.|}

Melania fontinalis, Philippi, Abb. III, 1851, "Pulo Pinang, in aquaducti. bus" [not of Brot].

6 Penang; coll. Dr. F. Stoliczka.

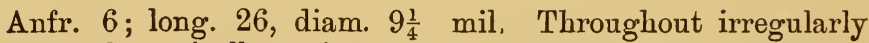
and acutely, spirally striate, striæ markedly more prominent towards the apex, without any longitudinal sculpture either in the adult or young forms. Of very sombre, uniform coloration; rather strongly decollate. Spire more produced; whorls less convex than in Philippi's figure: M. Brot figures as $M$. fontinalis some quite different species, longitudinally prominently ribbed-a character that exists neither in Philippi's description nor figure. I fancy it will prove not separable from the Javanese $M$. rivularis, which Philippi, indeed, himself suggested; my specimens, indeed, in shape more nearly resemble his figure of the latter. $M$. rivularis is united by M. Brot to $M$. tuber- 
culata; though very closely allied, I consider them perhaps separable. It is also very near $M$. nevilli, Brot.

subspecies malayana.

M. tuberculata, var. malayana, Issel, Ann. Mus. Civ. Genova, VI, 1874long. 13 ?, lat. 6 mil., Sarawak; fide Brot, distinct species.

6 Sarawak [typical]; coll. Prof. A. Issel.

Not at all like M. Brot's fig. 5, fairly represented by his $5 \mathrm{~A}$., perhaps, the real $M$. gemmulata of Reeve. The thick substance, planulate whorls, and developed longitudinal sculpture seem the best characteristics of the form.

\section{Melania [Striatella] rivularis.}

Melania rivularis, Philippi, Abb. II, pl. IV, fig. 6, 1847, Java.

20 Andamans; coll. F. A. de Roepstorff, Esq.

Long. $19 \frac{3}{4}$, diam. $6 \frac{3}{4}$; anfr. 7 .

Perhaps scarcely separable from subspecies fontinalis, Philippi. Some of the above exactly resemble the type figure.

1 Java; coll. Baron F. von Richthofen.

var. subunifascialis, noy. [? dist. sp.].

2 Andamans [type var.]; coll. F. A. de Roepstorff, Esq.

A very remarkable form; will perhaps prove specifically separable. I should have classed it as a var. of $M$. unifascialis, Mousson, from Java, but for the entire absence of longitudinal sculpture and the rather more convex upper whorls; the spiral striation is more or less obsolete on the upper portion of the whorls, imparting a very characteristic appearance to the shell; basal band round the columella not visible within the aperture.

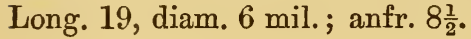

\section{Melania [Striatella] commersoni.}

Melania commersoni, Morelet, Ser. Conch. II, Nov. 1860, Madagascarlong. 35, diam. 11 mil.; fide Crosse from Rodriguez.

4. Mauritius ; coll. J. Caldwell, Esq.

14. Bourbon; coll. G. Nevill, Esq. 
Long. 28, diam. $10 \frac{1}{2}$ mil.; anfr. $6 \frac{1}{2}$.

Not unlike Troschel's Mel. adspersa.

var. minor [? M. tuberculata, var.].

10 Mahé, Seychelles; coll. G. Nevill, Esq.

A small, strongly decollate form, without longitudinal sculpture; appears to me fairly intermediate between the small, truncated Mauritian variety of $M$. tuberculata and typical $M$. commersoni.

Sub-Genus MELANOIDES, H.\& A. Adams.

Gen. Moll. I, page 296, 1854, type [as figured] Mel. asperata, Lam., as Genus Melanioides, Olivier.

I am unable to accept this section as of Olivier, in which case the type could evidently only be Mel. tuberculata, Müll. [=fasciolata, Oliv.]; I cannot find the word "Melanioides" in Olivier; and Férussac in his Tabl. syst. merely mentions a "Mélanö̈de, Olivier." Messrs. Adams included Mel. tuberculata in this subgenus, but M. Brot rightly; as I consider, separates it.

\section{Melania [Melanoides] reevei.}

Melania reevei, Brot, Mater. I, 1862,; $=M$. balteata, Rv. [not Philippi] $=$ M. goliath, Hanley, Con. Indica, pl. 72, fig. 3 ; var. $=M$. herculaa, var., Brot, Mat. II, pl. 3, fig. 3 ; fide Hanley [not Brot] juv. ? =M,

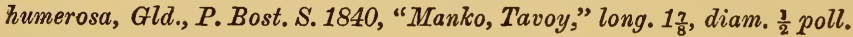

5 Pegu; ex c. Dr. F. Stoliczka.

Agree exactly with Con. Indica, pl. 72, fig. 3. Anfr. 4; long. $66 \frac{1}{2}$, diam. $32 \frac{3}{4}$ mil.

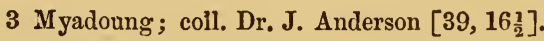

Apparently not quite adult; perhaps will prove a distinct, new variety.

var. lanceolata, nov.

Con. Indica, pl. 153, fig. 1, Burma.

M. Brot considers this the young state; I am inclined to think it an adult and very constant form. 
Long. 63, diam. $22 \frac{3}{4}$ mil.

20 Mandalay; coll. Dr. J. Anderson.

1 Henzada, Pegu; ex c. Dr. F. Stoliczka.

1 Thyet Myo; coll. $k$. Hungerford, Esq. [?].

This specimen should perhaps rather be classed in the typical

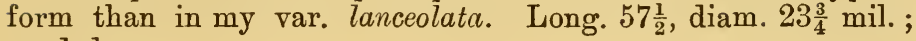
eroded.

A somewhat similar form frum Henzada exists also in $\mathrm{Mr}$. H. F. Blanford's collection.

\section{var. imbricata.}

Hanley, Con. Indica, pl. 153, fig.4 [Hab. ?].

1 Henzada; ex c. Dr. F. Stoliczka.

13 Yaylaymaw; coll. Dr. J. Anderson.

In quite young specimens, the "imbricated" sculpture can scarcely be detected. Long. 65 , diam. 25 mil. There are very fine specimens of this var. from Henzada in Mr. H. F. Blanford's collection, exactly resembling Brot's pl. 11, fig. 4A.

21 Thongyeen [Tenasserim Prov.]; coll. R. Hungerford, Esq.

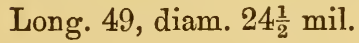

Strongly decollate, only three whorls remaining; excavated below the suture; longitudiual ribs obsolete on last whorl, strongly developed on the other two; in shape it closely resembles the typical form.

\section{var. soliduscula, nov.}

Brot, Conch..Cab. II, pl. 13, fig. 6, as "Mr. reveei [juv.]"; M. theobaldi, B. ms. in coll. H. F. Blanford.

10 Pegu [spec. juv.]; ex c. Dr. F. Stoliczka.

4. Noung-ben-Ziek; ex c. Asiatic Soc., Bengal.

M. Brot's above-quoted figure is not, as he supposes, a representation of the young of the typical form, which is altogether different; it is a nearly adult specimen of this very distinct variety, which is solid and light-yellow coloured; a quite young specimen having a single broad brown belt on the middle of the last whorl.

Long. 48, diam. 18 mil. 
var. [? M. humerosa, Gould].

1 Ataran [Tenasserim Prov.]; ex c. Dr. F. Stoliczka.

The whorls are markedly more convex in this specimen, and the spiral sculpture subobsoletely, but less unequally developed.

Long. $44 \frac{1}{2}$, diam. $17 \frac{3}{4}$; not decollate. Gould compares his species with $M$. intermedia, Busch.

\section{Melania [Melanoides] tourannensis.}

Melania tourannensis, Souleyet, Voy. Bonite, 1852, Touranne, long. 51, diam. 21 mil., decollate, 4 whorls.

Unlike M. Brot, I consider the deflection of the columellar margin as an important character of this species; his pl. 11, fig. $3 \mathrm{~b}$, and pl. 14, fig. 2 , may be compared.

\section{var. gloriosa.}

Melania gloriosa, Anthony, Amer. J. Conch., I, 1865, Pegu.

6 Saudoway District, Arakan; ex c. Dr. F. Stoliczka.

I have never seen as fine a specimen as that represented in the Con. Indica, pl. 72, fig. 2 ["Bassein"]; these Sandoway ones are intermediate between the above-quoted figure and fig. 6. Long. 66, diam. $30 \frac{1}{2}$ mil.

\section{var. peguensis [? dist. sp.].}

Melania peguensis, [Anthony], Hanley, Con. Indica, pl. 72, fig. 6.

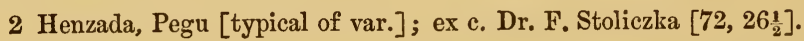

Almost seems to me specifically separate. Besides the different shape of the whorls and aperture, the entire absence of the spiral sculpture of the typical form is very characteristic.

var. compacta, nov.

2 Henzada, Pegu; coll. Dr. F. Stoliczka.

Long. $63 \frac{1}{2}$, diam. $23 \frac{1}{2}$ mil.; decollate, of 5 whorls.

This is the form nearest typical $M$. tourannensis, from which it principally differs by not possessing the excavation below the suture. It is a still smaller and more compact variety than var. peguensis, with the spiral striæ at base of last whorl very dis- 
tinct, much as in typical $M$. gloriosa, but more crowded; like the typical form, it is throughout regularly, though indistinctly, spirally striate. There are three specimens also in the collection of Mr. H. F. Blanford, and on the same tablet, presumedly from the same locality, "Henzada," a single specimen of $M$. reevei. It is very interesting to find the two species living together; this occurrence is confirmed by the specimens from Dr. Stoliczka's collection, wherein $I$ found the two above-recorded specimens and a young $M$. reevei mixed together.

var. beddomeana, nov. [? dist. sp.].

5 Near Moulmein; coll. Colonel R. H. Beddome.

An exceedingly interesting form. Long. 44, diam. $17 \frac{3}{4}$ mil.; slightly decollate, of five whorls. Closely resembles the preceding in shape of the whorls and spiral striation at base of last whorl; relatively even more solid; appears to be perfectly smooth throughout, with the exception of the above-mentioned basal striation.

\section{Melania [Melanoides] herculæa.}

Melania herculæa, Gould, P. Bost. S.II, 1846, Tavoy River; Con. Indica, pl. 72, fig. 5, and var. sowerbyi, Hanley, l. c., pl. 109, fig. 7 ; var. $=M$. julieni, Brot.

50 Near Moulmein [Tenasserim Prov.]; coll. Baron F. von Richthofen and Herr O. Limborg.

The largest-long. $81 \frac{1}{2}$, diam. 33 mil.

Prof. Brot apparently does not know this species, as he confuses it with a mere local race of Mel. variabilis ; this he figures, pl. X, fig. 1, as "typical" Mel. herculoea. On the same plate, fig. b, he figures a young specimen of the true Mel. herculoca, and remarks quite correctly that it is the $M$. hercucla, var. sowerbyi, of the Con. Indica. Moreover, he redescribes, most excellently, a local form of it as a new species-M. julieni, Deshayes, MS., from Tonkin.

\section{Melania [Melanoides] variabilis.}

Melania variabilis, Benson, J. Asiat. S., V., 1836, "Rv. Gumti and Tolly's nullah near Calcutta," and figured Gleanings Science, II, pl. 7, fig. 1; =M. varicosa, Troschel, 1837, Ganges; var.=Mel. sumatrensis, Brot, 1874 Sumatra and Java, and M. brookei, Reeve,

Benson, l. c., "Testa elongato-turrita solidâ olivaceâ vel piceâ, sub epidermide albidâ; anfractibus convexis transverse liratis, 
longitudinaliter striatis et costatis; costulis anfractûs ultimi, superne nodulosis; apice plerumque truncato; suturis excavatis ; aperturâ intus violaceâ, columellæ quasi sinuatâ."

Also subvar., Benson, l. c., as var. A. from Calcutta. "Anfractuum inferiorum liris elevatis, nodulis elevatioribus."

The common typical Lower Bengal form is also the $M$. varicosa of Troschel ; it is well represented by the Con. Indica, pl. 109, fig. 2 ["Calcutta"] and M. Brot's pl. 10, fig. 1 d. I do not believe the specimen figured by Eydoux and Souleyet really came from the Ganges.

100 Calcutta, Pt. Canning, \&c. ; ex c. Asiatic Soc., Bengal, J. B. Baxter, and G. Nevill, Esqrs.

1 Locality ?; ex c. Karachi Museum.

1 Kissengunj: ex c. Dr. F. Stoliczka.

Benson's original figure was, I suspect, taken from a specimen from this locality.

subvar. aspera.

Hanley, Con. Indica, pl. 109, fig, 6, and Benson, l. c., var. A. Calcutta.

Exceedingly close to Mousson's Mel. infracostata from Java.

16 Calcutta; coll. G. Nevill, Esa.

1 Calcutta ; ex c. Dr. F. Stoliczka.

subvar. cincta.

Hanley, Con. Indica, pl. 109, fig. 5, Assam.

3 "India "; ex c. Madras Museum.

2 Raniganj; coll. Dr. F. Stoliczka.

Interesting specimens, the ribs showing a strong tendency to become "spinose," as in M. episcopalis, Lea.

30 Calcutta ; ex c. Asiatic Soc., Bengal, and G. Nevill, Esq.

subvar. subtuberculata.

4 Calcutta ; ex c. Dr. F. Stoliczka.

Aperture relatively very small, almost round; last two whorls with scarcely any sculpture.

Long. 36, diam. $12 \frac{1}{2}$ mil. 
subvar.

6 Assam ; ex c. Dr. F. Stoliczka.

7 Bhootan; ex c. Dr. Stoliczka.

5 Teria Ghat; ex c. Dr. F. Stoliczka.

\section{subvar. subspinosa.}

5 Tank opposite Bengal Club, Calcutta ; coll. G. Nevill, Esq.

\section{var. infracostata.}

Melania infracostata, Mousson, Moll., Java, 1849, pl. 10, fig. 3.

3 Java; coll. Baron F. von Richthofen.

Unfortunately not adult. Two are the young of a form resembling that represented in Mousson's Moll., Java, pl. 10, fig. 4 [as " $M$. varicosa, Troschel "],-the whorls having longitudinal, distant ribs, on one specimen; these are quite obsolete on the last whorl, which, however, has a few spiral striæ below the suture; the third specimen shows similar longitudinal ribbing on the upper whorls, but the last whorl is spirally decussately striated, exactly as in the above-quoted figure of Mousson.

\section{var. subvaricosa, nov.}

14. Arakan and Pegu [?] ; ex c. Dr. F. Stoliczka.

Long. $65 \frac{1}{2}$, diam. 24 mil.

Reeve's fig. 10 A, "M. corrugata, Lamarck, India, \&c.," may, perhaps, represent this form, though it has the semi-nodulose sculpture a little more developed. M. Brot's pl. 10, fig. la, is close, but doubtfully identical; unfortunately, as in many other instances, he does not record the exact locality. The present form is more decollate, the ribs are straighter, a trifle further apart and more developed. The only figure that well represents it is that of the Voy. Bonite, pl.31, figs. 11-12, la "Melania indienne" from the Ganges, = Melania varicosa, Troschel, in text, 1. c., not $M$. indica, Soul., as stated by Brot, a name I cannot find in the Voy. Bonite. There seems to be a gradual transition to the $M$. episcopalis, Lea, which lives in the same neighbourhood.

10 Near Sibsagar; coll. S. E. Peal, Esq.

With sculpture not unlike that of $M$. infracostata, Mousson. It is, I think, the form figured by Brot, pl. 10, fig. 1c.

10 Near Sibsagar, Namtsik River, and Samaguting ; coll. S. E. Peal, Esq. 
Longitudinally, somewhat indistinctly ribbed, ribs flexuous, not, so far as I can see, decussated by any transverse lyræ.

10 Kusiyara Rv., \&c., Sylhet ; coll. J. IVood.Mason, Esq.

3 Sudiya, near Darjiling; coll. Colonel G. B. Mainwaring.

Unfortunately not adult, so that I am unable to feel sure of having correctly classified the form.

var. semilævigata, nov.

Benson, J. A. S. B., 1836, as M. variabilis, var. D. Syllet [ex c. A. S. B.] ; Brot, Conch.-Cab.II, 1874, pl. 13, fig. 1, as "MI. sumatrensis, Brot, var."

Long. 56, diam. $24 \frac{1}{3}$ mil. ; four whorls remaining.

Var. D. Benson, 1. c., " Anfractuum superiorum costulis obsoletis, ultimi et penultimi liris transversis costulis longitudinalibusque superne serie duplici nodulosis."

A very interesting form, admirably described by Benson, moderately decollated, upper whorls perfectly smooth, last two girt with a double row of prominent regular nodules, more or less subobsoletely spirally striate at base of last whorl, one rib about the centre [starting from top of the aperture] always prominent, the two rows of nodules are joined by longitudinal ribs, more or less obsolete; the above described sculpture of the two last whorls is sometimes nearly or partially obsolete; in not a single specimen do I find traces of sculpture on the upper whorls. Brot's above-quoted figure is a fair representation of the form ; in shape it is exactly the same. Cachar specimens are more decollate, have the sculpture terminating even more abruptly, nodules a little more distinct; it is very interesting to find this characteristic variety repeating itself, if I may use the expression, in Sumatra. I need scarcely say M. Brot's other figures of his $M$. sumatrensis (except his pl. 13, fig. $1 \mathrm{~b}$, which may be the young) represent widely different forms.

15 Cachar and Sylhet [type var.] ; ex c. Asiatic Soc., Bengal.

The $M$. infracostata, Mousson, from Java and $M$. brookei from Borneo may possibly all prove to belong to this variety, as mere local races; slight differences in sculpture of the last two whorls being their only distinguishing features, apparently.

30 Doarbund, Silcuri, Cachar ; coll. J. Wood.Mason, Esq. 


\section{var. hainesiana.}

Melania hainesiana, Lea, Proc. Acad. Nat. Sci., Philad., 1856, and Observ., pl. 22, fig. 18, India; Benson, J. A.S. B., 1836, pl.746, as M. variabilis, var. C.from Sylhet.

Benson, 1. c. "Laevis liris costulisque obsoletis, anfractûs ultimi medio subcarinato, adulti nodulis humeralibus frugaliter sparsis." This form is not figured in the Con. Indica, or in Reeve; Brot merely gives a copy of Lea's figure, and that a bad one, as he makes the basal sulcations far too developed. Lea, in his description, notes that "one of his younger specimens has a disposition to indistinct folds towards the apex."

8 Khasi Hills, N. Cachar ; coll. Colonel H. H. Godwin-Austen.

Agree exactly with original figure, which is, however, that of a not quite adult specimen.

1 Dihiri Hill [Brahmaputra watershed]; coll. Colonel H. H. GodwinAusten.

Less characteristic and nearer typical M. variabilis; subobsolete longitudinal ribbing being distinctly traceable, the last whorl less distinctly angulate, and the spire more produced.

16 Tezpore and Gowhatty [Assam]; coll. Dr. F. Stoliczka, \&c.

Spire produced, longitudinal ribbing almost or altogether obsolete, basal sulcation subobsolete; a single specimen is very interesting, showing the transition to my var. semilcevigata, there being five small, but distinct, nodules behind the outer lip, midway between the peripheral keel and the suture.

1 Sibsagar ; coll. S. E. Peal, Esq.

Like the above Tezpore specimens, but the last two or three whorls are biangulate, or rather bi-carinate.

4 Sylhet; ex c. Asiatic Soc., Bengal [? typical M. variabilis-var. C. of Bs.].

One of these is also bicarinate on the last whorl.

2 Gowhatty; coll. Museum Collector.

3 Naga Hills; coll. A. W. Chenne!l, Esq.

16 Gowhatty; coll. Museum Collector.

This is the most distinct and interesting form of the variety that I have seen; it is of unusually thick substance, not decollate; spire peculiarly short, of 10 whorls; uniform bright 
green, the extreme young only showing two basal brown bands; distinctly angulate at the periphery, basal sulcations rather more distinct than usual; nearly smooth, but subobsolete sculpture can be traced here and there; outer lip above strongly deflected, at base acuminately pointed, much as in $M$. reevei; both this and subvar. fasciata from Bhootan are probably transition forms to $M$. reevei, Brot.

Long. 44, diam. 18 mil.

subvar. fasciata.

4 Bhootan ; ex c. Dr. F. Stoliczka.

This may prove an altogether distinct variety. Decollate, of four whorls only, long. 33, diam. 17 mil. Quite adult, with a somewhat indistinct brown band in the middle of the whorls, two at base; substance rather thin, outer lip not acutely angled at base, although angled at the periphery; no tendency to possess a raised keel there, as is often the case.

subspecies episcopalis.

Melania episcopalis, Lea, P. Zool. S. 1850, Malacca; Hanley, Conch. Misc., pl. III, fig. 27 ["typical specimen"] ; Brot, Conch.-Cab. II, pl.12, fig. 1, as "M. episcopalis" and l. c. fig. 4 [also 4A. and B.], as "M. brookei," and l. c. pl. 13, fig.1b, as "M. sumatrensis, var.; subvar. = Hanley, Con. Indica, pl. 75, fig. 6, as "M. variabilis, var. spinosa [not M. spinosa, Hanley, as of Benson, Conch. Misc.]; Reeve, fig. 207, as "M. brookei" and fig. 208, as "M. sparsinodosa, Busch" [both from Borneo]; = M. pontificalis, Busch, Zeits. Mal. 1853, Borneo.

This widely spread and variable form is not the Mel. episcopalis of the Con. Indica, which I have named var. binodulifera. Why M. Brot would not accept Hanley's typical figure in his Conch. Misc. of $M$. episcopalis, which nevertheless agrees fairly not only with the original description, but also with Brot's Malacca specimen [pl. 12, fig. 1] and my Perak specimens from the same district, I am quite unable to understand; possibly, the subsequent series of errors in the Con. Indica induced the Professor not to do so. These errors are that Hanley figures the episcopalis of Lea for his own Mel. spinosa, and the true spinosa as "M. menkiana, Lea" [and subvar. as $M$. hanleyi, G.-A.].

8 Qualla Kangsa, Perak; coll. Surgeon-Major E. Townsend. 
Though in bad condition, some of these agree exactly with above-quoted fig. 27, Conch. Misc., and others with Brot's pl.12, figs. 1 and 4. Two of these specimens are banded, as in the sub. var. cincta of typical $M$. variabilis.

Long. 5l, diam. 24; 5 whorls.

1 Qualla Kangsa ; coll. Surgeon-Major E. Townsend.

A not adult specimen, apparently of Brot's pl. 12, fig. 1A, ribs slightly binoduloso above, two distinct brown bands at base.

3 Perak; coll. Surgeon-Major E. Townsend.

A smaller form, unfortunately in very bad condition; it will probably have eventually to be separated, and may rather belong to var. menlieana.

4. Tongoop ? [Arakan]; ex c. As. Soc., Bengal.

Exceedingly close to the Samaguting specimens. One of the Perak specimens also has very similar, distant, large ribs.

1 Henzada? [Arakan]; ex c. Dr. F. Stoliczka.

1 Naga Hills; coll. A. W. Chennell, Esq.

5 Teria Ghat; coll. Colonel H. H. Godwin-Austen.

Apparently a very variable small form; one or two of them closely resemble Perak typical episcopalis. The absence of any basal striation connects the form with $M$. menlieana, Lea.

8 Teria Ghat ? ; ex c. Dr. F. Stoliczka.

Locality I consider doubtful. It is very close to $\boldsymbol{M}$. variabilis, var. subspinosa [Calcutta].

5 Sibsagar [?], \&c.; ex c. Dr. F. Stoliczka.

A small form near the preceding, no basal striation.

5 Khasi Hills; coll. Colonel H. H. Godwin-Austen.

This fine form resembles the one I have separated (perhaps injudiciously) as subvar. binodulifera; pl. 75, fig. 7 of the Con. Indica, would fairly represent it, but that the spiral ribbing is almost obsolete in all my specimens.

2 Munipur; coll. Colonel H. II. Godwin-Austen.

A less conspicuous form than the preceding, which it otherwise resembles. 
subvar. intermedia.

Brot, Conch.-Cab. II, pl. 13, fig. 11, as M. spinosa, var. B.

3 Tezpore [Assam]; coll. Dr. F. Stoliczka.

Excellently represented by the above quoted figure. As M. Brot justly remarks [1. c. page 93], it is an interesting and important transitional form between typical $M$. variabilis and $M$. episcopalis.

\section{var. pseudospinosa.}

Nevill, J. A. S. B. 1881, pl. VI, fig.'17, Assaim; Con. Indica, pl. 75, fig. 6.

I can detect no good character to distinguish this form from Perak and Malacca specimens; the spire is usually more produced and less decollate, the ribs more spinose at the angle, which, again, is more central.

15 Cachar; coll: Museum Collector.

Fairly intermediate between M. Brot's pl. X, fig, $2 \mathrm{~B}[$ [ $M$. sumatrensis"] and his pl. 12, fig. 2 ["M. spinosa"]; upper whorls longitudinally ribbed.

Long. 68, diam. 24 mil.; 9 whorls.

6 Tezpore; coll. Dr. F. Stoliczka.

One or two of these are scarcely separable from my Perak typical episcopalis. It is possible they had better been ciassed in subvar. pontificalis ; the sculpture of the upper whorls in all of them is very superficial.

2 Assam; ex c. Dr. F. Stoliczka.

Not quite adult; may be the young of M. Brot's pl. 10, fig. IA ["M. variabilis"], perhaps better classed as var. subtypica, subvar. subinfracostata.

17 Namtsik River, Sibsagar and Samaguting [Assam]; coll. S. E. Peal, Esq., and Capt. Butler.

Agree fairly with Con. Indica, pl. 75, fig. 6 ["M. spinosa "], one specimen (undoubtedly of the same stock) is very interesting, as the last whorl is smooth.

5 Sundoway River [Arakan]; ex c. Dr. F. Stoliczka. 
A very fine form, with the last whorl and aperture unusually tumidly swollen. Long. 81, diam. 34 mil.; 7 whorls.

10 "Hlowa Choung", Arakan Hills; ex c. Dr. F. Stoliczka.

These agree exactly with Con. Indica, pl. 75, fig. 6, and are therefore typical of subvar. pseudospinosa. Long. 86, diam. 29 mil.

The locality given is somewhat untrustworthy.

var. binodulifera, nov.

Con. Indica, pl. 72, fig. 7, and pl. 75, figs. 5 and 7, N. Cachar, as M. epis. copalis, Lea; fide Brot, M. spinata, Godwin-Austen, var.

5 Khasi Hills; coll. Colonel H. H. Godwin-Austen.

Four agree exactly with the above-quoted fig. 5 ; the fifth is of interest, as it connects the form with var. sublcevigata [Sylhet]; the last $2 \frac{1}{2}$ whorls only possessing the characteristic sculpture. Long. 75, diam. 34 mil.

5 N. Cachar; coll. Colonel H. H. Godwin-Austen, and ex c. Asiat. Soc., Bengal. mil.

A short form allied to the preceding. Long. 46, diam. 24

1 N. Cachar; coll. Colonel H. H. Godwin-Austen.

A beautiful little form, like a miniature of Con. Indica, pl. 75, fig. 5 , but with compressed, narrow, last whorl and aperture; scarcely decollate. Long. 42, diam. $16 \frac{1}{4}$ mil. ; 6 whorls with a double row of very distinct nodules, of almost equal size.

30 Barak River, Silchar; coll. J. Wood-Mason, Esq.

Like the preceding, but a rather smoother form.

6 Diyung River, Assam; coll. Colonel H. H. Godwin-Austen.

The form well represented in the Con. Indica, pl. 72, fig. 7.

var. pontificalis.

? Melauia pontificalis, $v . d$. Busch.

4 Near Sibsagar; coll. S. E. Peal, Esq, 
A remarkably solid, heavy form; upper whorls smooth, the last (and sometimes the last two) with a row of ill-defined longitudinal ribs from the centre of the whorl to its base, developed into spinose tubercles above; closely resembles $M$. Brot's pl. 12, fig. 4A ["M. brookei, var. pontificalis"], but the tubercles are more regular and numerous and placed a little more centrally. Long. 72, diam. $24 \frac{1}{2}$ mil.

Reeve gives $M$ Mel. pontificalis as a synonym of Mel. infracostata from Borneo.

var.

3 Ceylon [?] ; coll. E. L. Layard, Esq. [A. S. B.].

A very interesting and distinct form, although I cannot help doubting the correctness of the label- "Ceylon."

I have never seen the species elsewhere, either from that island or from South India.

subspecies menkeana [emend.].

Melania Menkiana, Lea, Trans. Amer. Philos. Soc. 1842, for Mel. plicata, Lea, Trans. Am. Philos. Soc. VI [1835, fide Lea], not of Menke, 1829, "India ?"; = M. spinosa, (Benson), Hanley, Conch. Misc. 1856, pl. 1, fig. 7 [not Mel. spinosa, "Benson," of Brot. Conch..Cab. II, or Mel. variabilis, var. spinosa, Hanley, Con. Indica, pl. 75, fig. 6] ; = Con. Indica, pl. 110, fig. 6, M. menkiana Lea, "Khersasip, N. Cachar"; $=M$. variabilis, Benson, var. B., J.A. S. B. V, 1836, Sylhet; var.= M. godwini, Brot, Conch.-Cab. II, 1874; for Mel. hanleyi, G.-Aust. 1872, N. Cachar, Diyung river [not Mel. hanleyi, Brot].

Benson's description of $M$. variabilis, var. B. is-" Liris, medianâ exceptâ, obsoletis ; nodulis subspinosis carinam humeralem coronantibus."

When Mr. Hanley issued plate 75 of the Con. Indica (see page $32,1$. c.), he evidently had not discriminated between the two forms represented by his two above-quoted figures ; on page 45 , however, he correctly separates the form which Lea described as $M$. menkiana $[$ not $M$. menkeana as stated by $M$. Brot, who incorrectly charges Mr. Hanley with the error] and states(which is also undoubtedly the case) that it is the same form as that originally figured by him in the Conch. Misc. as $M$. spinosa, Benson; Lea's figure is nearer Con. Ind. pl. 110, fig. 5 [ $\boldsymbol{M}$. godwini, Brot] than fig. 6 [M. menkeana]; unfortunately, M. Brot adopts the name of $M$. spinosa, Bs., for pl. 75, fig. 6 
of the Con. Indica; but the type of M. spinosa, Benson, must remain the shell figured in the Conch. Misc. [though not described] which we all unite in considering $=M$. menkeana.

7 Sylhet [typical var. B. of Beuson $=M$. spinosa, Hanley 1856]; ex c. Asiat. Soc., Bengal.

Long. $22 \frac{1}{2}$, diam. $13 \frac{1}{2}$ mil.

30 Teria Ghat, \&c. [Khasi Hills]; coll. Colonel H. H. Godwin-Austen, \&c.

Long. $34 \frac{1}{2}$, diam. 17 mil.

subvar. microstoma.

A small and remarkable form, apparently from the same locality as typical var. B. of Benson, from which it can be distinguished by the small and almostrounded aperture, the more produced and perfect spire, and whorls less convexly swollen; two brown bands at the base, as in typical var. B., but the upper whorls also. possess a distinct central band; two specimens have subnodular ribs on the last two whorls, the upper ones being perfectly smooth, the third specimen has nodulose ribs on the three last whorls, the fourth has them on the last whorl only; these nodulose ribs are variable in number and relatively distant.

Long. 25, diam. $11 \frac{3}{4}$ mil.

10 Sylhet; ex c. Asiat. Soc., Bengal.

\section{Melania [Melanoides] spinata.}

Melania spinata, Godwin-Austen, P. Zool. S. 1872, Kopili River.

This remarkable form, excellently figured by the author [pace M. Brot], preserves its characteristics in both young and adult. M. Brot makes the strange error of supposing it might be the young of the form figured [correctly] as M. episcopalis, Lea, in the Con. Indica; I have both forms in all stages, and need scarcely say that they are widely different. Possibly, indeed, it might also be classed as an extreme variety of $M$. variabilis ; the peculiar, "foliaceous" tubercles are very characteristic. It bears also some resemblance to a form of Mel. baccata, Gld.

8 Kopili River, N. Cachar ; coll. Colonel H. H. Godwin-Austen. 


\section{Melania [Melanoides] baccata.}

Melania baccata, Gould, P. Bost. S. 1847, River Thoungyin.

Reeve's fig. 1, M. henriettce, Gray, from China, seems to me different and specifically separable.

3 Upper Salwin [Shan States]; coll. F. Fedden, Esq.

Resemble Con. Indica, pl. 75, fig. 3 [var. pyramidalis, Th.]; one of them has a single row of spinose nodules in the middle of each whorl instead of three; this agrees exactly with Theobald's type.

subvar. recta.

Brot, pl. 9, fig. 6, as M. baccata, Gld.

1 Upper Salwin; coll. F. Fedden, Esq.

Well represented by the above figure, but more decollate, only $2 \frac{1}{2}$ whorls remaining.

var. iravadica.

Melania iravadica, W. T. Blanford, P. Zool. S. 1869, "Malé and Bhamo."

There can be, I think, no question as to the correctness of uniting this form to $M$. baccata, despite its smaller size and less conspicuous sculpture, which is, however, of an exactly similar nature. Long. 30, diam. 17 mil.

30 Manwyne [type var.] and Yaylaymaw [Upper Burma]; coll. Dr. J. Anderson.

\section{Melania [Melanoides] subasperata, nov.}

Theobald, J. Asiat. S. Beng. 1865, pl. 9, fig. 5, as M. variabilis, var. baccifera, Shan States, Nammah Stream.

1 Shan States ; coll. F. Fedden, Esq. [47, 17].

This is a typical var. baccifera, Th. ; it is very close to $\mathrm{M}$. Brot's pl. 9, fig. 4A ["M. perfecta, Mss., Celebes"] and also to his pl. 8, figs. 1 and $1 \mathrm{~B},[M$. asperata, Lea, Philippines], only smaller; the shape of the spire more nearly resembles that of $M$. variabilis than $M$. baccata; the small and contracted, almost rounded aperture seems to be characteristic. 
var. sublævigata, nov.

2 "Burma" ; ex c. Dr. F. Stoliczka.

1 Shau States; coll. F. Fedden, Esq.

Well represented by M. Brot's, pl. 3, fig. 5 [M. subnodosa, Phil., South America], only a little smaller.

var. vittata.

Theobald, J. As. S. Beng., 1865, fig.4, as M. variabilis-var. vittata.

1 Shan States; coll. F. Fedden, Esq.

\section{Melania [Melanoides] cancellata.}

Melania cancellata, Benson, Ann. Mag. 1842, Chusan; = M. ningpoensis, Lea, P. Ac. Nat. Sci. Philad.1856, Ningpo;=M. fortunei, Reeve, 1859, Shanghai; fide Brot=M. amurensis, Gerstfeld=M. heukelomiana, Reeve var. $=$ M. calculus, $R v$. [N. China $]$.

3 Chusan [typical ?] ; ex Mr. Damon [c. Cantor].

2 (bina ; ex c. A. Morelet, Esq.

2 Khasing District [N. China]; coll. R. Hungerford, Esq.

3 Ningpo; ex c. Dr. H. Dohrn.

$M e l$. admiralis, Smith, from Lake Tanganyika, seems to show some affinity to this species.

\section{Melania [Melanoides] amurensis.}

Melania amurensis, Gerstfeld, Mém. Ac. Petersb. IX, 1859, Rv. Amur, long. 45 diam. 16 mil. and var. lavigata, long. 20 , diam. $8 ;=\mathbb{M}$. heukelomiana, Rv., 1859, Hab. ? [39, 15] ; ? var.=M. calculus, Rv., 1859, N. China.

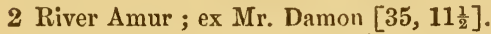

1 River Siangkiang [N. China]; ex c. Dr. H. Dohru.

subvar. sublævigata.

1. River Siankiang; ex c. Dr. H. Dohrn $[23,9]$.

46. Melania [Melanoides] crebricostis.

Melania crebricostis, Benson, Ann. Mag. 1842, Chusan, long. 1.05 poll.

6 Kowloon [opposite Hongikong] ; coll. R. Hungerford, Esq.

1 Amoy? ; coll. R. Hungerford, Ésq. 
47. Melania [Melanoides] niponica.

Melania niponica, Smith, Quart. Journ. Conch. I. Lake Biwa.

5 Biwa Lake, Japan; coll. R. Hungerford, Esq.

48. Melania [Melanoides] schomburgki.

Melania schomburgki (Hanley), Reeve, Icon. fig. 93, 1859, Hab. ?

8 Cambodia ; cx c. A. Morelet, Esq.

\section{Melania [Melanoides] torquata.}

Melania torquata, v. d. Busch, Abb. I, 1845, Java;=M. terebra, Benson, 1836, Sylhet [not M. terebra, Lesson, 1830, or M. terebra, Busch, 1845].

10 Sylhet [types of $M$. terebra, Bs.] ; ex c. Asiatic Soc., Bengal, and Colonel G. B. Mainwaring.

\section{Melania [Melanoides] asperata.}

Melania asperata, Lamarck, Hist. Anim. s. Vert. V, 1822, South America?

I consider Reeve's fig. 18, "M. circumstriata from Borneo," as quite distinct and probably a form of $M$. herculoea, Gld.

8 Near Manila; coll. Baron F. von Richthofen.

This apparently constant dwarf form more nearly resembles M. Brot's pl. 9, fig. 4, $M$. perfecta, Mous., from Celebes, than his pl. 8, fig. 1A, typical $M$. asperata,-indeed, it may prove to be the former species. Long. 35, diam. 14; another $22,10 \frac{1}{2}$ mil.

Living with it was the following subvariety distinguished by its smooth appearance, having spiral, subobsolete sulcations on the last whorl only.

subvar. sublævigata.

4. Near Manila; coll. Baron F. von Richthofen.

Three or four irregular ridges on the last two whorls. The upper ones smooth [or nearly so].

Long. 56, diam. 22 mil.; whorls $5 \frac{1}{2}$.

2 Pasanhan [Luzou]; coll. R. Hungerford, Esq. 
var. inquinata.

Melania inquinata, Deshayes, Mag. Conch.pl. 13, 1830, Philippines.

1 Pasanhan; coll. R. Hungerford, Esq.

Whorls more convex than in any of the other specimens.

var.

Conf. Reeve, fig. 2 c, "M. asperata."

6 Luzon [?] ; coll. Baron F. von Richthofen.

2 Hasanlan; coll. R. Hungerford, Esq.

Whorls less convex than in any others I have seen; the peripheral keel is more developed than the others. It may possibly be a form of $M I$. dactylus.

rar.

Something like Reeve's fig. 2 d., but on a much smaller scale.

2 Pasanhan ; coll. R. Hungerford, Esq.

var.

Like M. Brot's pl. 8, fig. lf., but smaller.

5 Pasanhan, \&c. [Luzou] ; coll. Baron F. von Richthofen, and R. Hungerford, Esq.

\section{Melania [Melanoides] lacustris.}

Melania lacustris, Morelet, Test. nov. I, 1849, Lake Yzabal [Guatemala].

1 "Lac d" Yzabal" [typical]; coll. A. Morelet, Esq.

Agrees with M. Brot's pl.3, fig. 2c, and Hanley's Conch. Misc., fig. 26.

52. Melania [Melanoides] dactylus.

Melania dactylus, Lea, P. Zool. S. 1950, Philippines.

5 Philippines; coll. Prof. C. Semper.

Sent as " $M$. asperata, var. dactylus"; they are a rather smaller form, otherwise agreeing well with M. Brot's pl. 9, fig, 2.

2 Cebu ; ex c. R. Hungerford, Esq. [coll. Dr. Eastlake]. 
Resembling Reeve's fig. 7a, but smaller.

1 Cebu; ex c. R. Hungerford, Esq. [coll. Dr. Eastlake].

A form intermediate between this species and Mel. asperata; the last whorl [only] binoduliferous, as in Reeve, fig. 7b.

\section{Melania [Melanoides] filocarinata.}

Melania filocarinata, (Mousson), Brot, Conch.-Cab. II, 1874, Polillo; long. 62, diam. 21 mil.

5 San Palo, Luzon; coll. R. Hungerford, Esq.

Agree exactly with the type figure.

var. subimbricata, nov.

1 San Palo; coll. R. Hungerford, Esq.

Long. 49, diam. 16 mil. A variety bearing much the same relationship to the type form, as does M. Brot's pl. 9, fig. 2A to its type [M. dactylus]; apical six whorls smooth.

\section{Sub-Genus SULCOSPIRA, Troschel.}

Brot, Conch.-Cab. I, 1874, type M. sulcospira.

\section{Melania [Sulcospira] libertina.}

Melania libertina, Gould, P. Bost. Soc. VII, 1859, Simoda and Ousima = M. tenuisulcata, Dkr. Malac. Blät. 1859, =M. reiniana, Brot, Jahrb. 1876, Japan; var. decussata, Mts., l. c. Japan; var. irrigua, Mts., Sitz. Fr. Berl. = Yokohama; fide Brot, = Mr. doria, T.-Canef.

4. Hakone, Japan; coll. R. Hungerford, Esq.

10 Tamsui, Formosa; coll. R. Hungerford, Esq.

\section{var. sublævigata.}

12 Tamsui, \&c.; coll. R. Hungerford, Esq.

Perhaps the var. ambidextra of Martens [Japan]. Anfr. 4, long. 25, diam. 12 mil. 
var. plicosa.

Mel. libertina, var. plicosa, v. Martens, Malac. Blät. 1860, Japan; Kobelt, Fauna Japan. pl. 18, figs. 2-5.

10 Mai Tionlek, Formosa ; coll. R. Hungerford, Esq. [28, 13].

2 Hakone, Japan; coll. R. Hungerford, Esq.

var. microstoma, nov.

10 Formosa; coll. R. Hungerford, Esq.

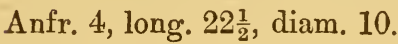

var. subplicosa.

18 Formosa ; coll. R. Hungerford, Esq.

The $M$. plicifera, Tryon, from Japan appears to be very close, also Kobelt's pl. XVIII, figs. 6-8 ; and the latter's pl. XIX, fig. 8, again, exactly represents some of the specimens. Anfr. 4 ; long. $24 \frac{1}{2}$, diam. $10 \frac{1}{2}$ mil.

\section{var. japonica.}

Melania japonica, Reeve, Icon. fig. 125, 1859, Japan; dist. sp. fide Brot and $=$ Mel. ambidextra, Mts., Mal. Bl. 1860, Japan.

1 Japan; ex c. Colonel G. B. Mainwaring.

6 Nikko, Japan; coll. R. Hungerford, Esq.

Resemble Kobelt's pl. XVIII, fig. 6, stated to be the Mel. libertina, var. decussata of Martens.

\section{var. irrigua [?].}

Melania libertina, var. irrigua, Martens, Sitz. Ges. Naturh. Fr.; Yokohama.

7 Meanoshita, Japan; coll. R. Hungerford, Esq.

Well represented by Kobelt's pl. XIX, fig. 4 .

var. doriæ [?].

Melania doriæ, Tapparone-Canefri, Voy. "Magenta," Moll, pl. I, fig. 4, Singapore [Hab. erron.].

3 Nagasaki, Japan; coll. R. Hungerford, Esq. 
Unfortunately not quite adult. A large, tumid form, apparently even more aberrant than the above-quoted figure, or than any of the varieties figured by Kobelt.

Sub-Genus NIGRITELLA, Brot.

Conch.-Cab. II, 1874, type M. nigritina, Morelet.

55. Melania [Nigritella] guineensis.

Melania guineensis, Reeve, Icon. fig. 142, 1860, " Coast of Guinea."

var. minor.

4. Liberia ; coll. Dr. H. Dohrn [23, 9르.

56. Melania [Nigritella] decollata.

Melania decollata, Lamarck, Hist. 1822, Riv. de la Guyane; fide Brot= M. erosa, Philippi $[$ not Less $]=$ M. sculptilis, Reeve.

3 La Guayra; coll. A. Morelet, Esq.

Sub-Genus PACHYCHILUS, Lea.

P. Zool. S. 1850, p. 179, type P. cumingii, Lea [as Genus nov.].

57. Melania [Pachychilus] largillierti.

Melania largillierti, Philippi, Abb. I. 1845, Centr. America; fide Brot. .M. rusticula, Busch and M. rubicunda, Reeve.

var. intermedia.

Melania intermedia, von dem Busch, Philippi, Abb. I, 1845, "Lacus Nicarigua."

2 Guatemala; coll. A. Morelet, Esq.

\section{Melania [Pachychilus] conica.}

Melania conica, d'Orbigny, Hist. Nat. Cuba, 1841; fide Brot=M. nigrata Poey, Mém. Cuba II, 1852=M. scarabus, Rv., Icon., Hab. ?=M. gemella, Rv.; Icon., Cuba=M. attenuata, [Anthony], Reeve, Icon., Cuba.

5 Cuba ; ex c. Dr. F. Stoliczka [ex Landauer].

2 Cuba; ex c. Dr. F. Stoliczka [libelled " M. nigrata, Poej."] 


\section{Melania [Pachychilus] corvina.}

Melania corvina, Morelet, Test. nov. Cuba I, 1849.

1 Guatemala [typical] ; coll. A. Morelet, Esq.

A dwarf specimen, long. 22, diam. $12 \frac{1}{2}$ mil.

60. Melania [Pachychilus] lævissima.

Melania lævissima, Sou'erby, Zool.Journ. I, 1825, La Guayra; file Brol= M. indiorum, Morelet, "circa Minas Palengueanas =M. sallei, Reeve, T'enezuela.

var. clava.

Melania lævissima, var. clava (Menke), Brot, Conch.-Cab. II, 1874,pl. 4, fig. 5 d.

2 Venezuela ; coll. A. Morelet, Esq.

61. Melania [Pachychilus] brevis.

Melania brevis, d'Orbigny, Hist. Nat. Cuba, 1841.

5 Cuba ; ex c. F. Layard, Esq. and Dr. F. Stoliczka.

\section{Melania [Pachychilus] testudinaria.}

Melania testudinaria, von dem Busch, Philippi, Abb. I, 1S45, Java; vars. lutea, scalaroidea and striatula, Mousson, Moll., Java, 1849.

3 Java; ex c. A. Morelet, Esq.

$$
\text { var. olongata, nov. }
$$

30 Java; coll. Baron F. von Richtlıofen.

Long. $42 \frac{1}{2}$, diam. $15 \mathrm{mil}$.

$$
\text { var. subangulata, nov. }
$$

2 Java; coll. Baron F. von Richthơfen.

\section{Melania [Pachychilus] limborgi.}

Melania limborgi, Hanley, J. Linnean, Soc., XIV,18\%9. p. 5SO, Tenasserim.

3 Near Moulmein [Tenasserim Prov.] ; coll. Mr. Oscar Limborg. 
64. Melania [Pachychilus], n. sp.

3 Pegu ; ex c. Dr. F. Stoliczka.

65. Melania [Pachychilus] hungerfordiana, n. sp.

Long. $39 \frac{1}{2}$, diam. 16 mil.

Closely resembles M. Brot's Mel. citrina, pl. 13, fig. 5, also Philippi's M. largillierti. Slightly truncate, seven whorls remaining, which in one specimen are distinctly more convex than in the other ; thick and solid, of a yellowish brown colour, girt with a single well-marked band in the middle of the upper whorls ; three bands on the last whorl; the basal margin subacutely angled, not rounded as in the above-quoted figure; no spiral sulcation at base of last whorl.

2 Upper Burma [type] ; coll. R. Hungerford, Esq.

5 Pegu [spec. juv.]; ex c. Asiatic Soc., Bengal.

Sub-Genus ACROSTOMA, Brot.

Conch.-Cab. II, 1874, type Mel. hügeli, Philippi.

66. Melania [Acrostoma] hügeli.

Melania hügeli, Philippi, Abb. I, 1845, Nova Hollandia ?;=M. siphonata, Rv., Hab. ?

3 Upper Cauvery River; coll. H. F. Blanford, Esq.

These agree well with figures in Phillippi, Reeve, and Con. Indica.

var. compacta, nov.

Long. 25 $\frac{1}{2}$, diam. $13 \frac{3}{4}$ mil.

Easily distinguished by its short, stout form, relatively contracted aperture, less tumidly swollen, but more subangulate last whorl, and closer spiral sculpture ; the spire is more trun. cate ; the coloration darker.

1 Wynaad [type var.]; coll. Colonel R. H. Beddome.

2 Cochin Hills ; coll. Colonel R. H. Beddome.

4 Cauvery River; coll. Surgeon.Major Jerdon [A. S. B.]. 
These latter were labelled "Mel. carnatica, Bens. MS.;" they present the distinctive characters of the variety even more strongly. Long. 25, diam. 14 mil.

\section{Melania [Acrostoma] assamensis, n. sp.}

Hanley, Conch. Misc. pl. 5, fig. 43, as "M. hïgelii, var. of Phil. from Khasya."

Long. 35, diam. $18 \frac{1}{2}$ mil.

Remarkably close to Mel. hïgeli, from which it can be distinguished by its less solid substance, its much more convexly rounded whorls, the last not being [more or less] subangulate as in its ally; of a plain, uniform, dark coloration; of similar minute striation, but wanting the spiral sulcations at base of last whorl ; characters of the columellar margin and aperture generally much as in typical $M$. hïgeli. Strongly decollate, three whorls only remaining.

4 North Cachar [type] ; coll. Colonel H. H. Godwin-Austen.

1 "Delaima " [?]; ex c. G. Nevill, Esq.

\section{Sub-Genus MELANELLA, Swainson.}

Treat. Malac. 1840, p.341 [no type recorded] ; type M. holandri, Fér.

68. Melania [Melanella] holandri.

Melania holandri, Férussac, Villa, Catal. 1841 [fide Reeve].

var. costulata.

Melania holandri, var. costulata, Schmidt, Syst. Verz. Krain, $1847 ;=\mathbb{M}$. semiplicata, Brusina, Contr. Mal. Croat. 1870.

5 Croatia; ex c. Colonel G. B. Mainwaring and P. Joly, Esq.

69. Melania [Melanella] brevicula.

Melaniella brevicula, H. Adams, P. Zool. S. 1S70, Amoy; I. Hainan [Geale] fide Brot.

3 Amoy [typical]; ex c. H. Adams, Esq.

\section{Melania [Melanella] glans.}

Melania glans, von dem Busch, Philippi, Abb. I, 1845, Java; juv., fide Brot $=M$. siccata, Busch, Java; from Philippines, Cuming [!].

10 Java [?] ; coll. Baron F. von Richthofen. 


\section{Melania [Melanella] zonata.}

Melania zonata, Benson, J. Asiat. Soc. Bengal, 1836, Sylhet; fide Brot?=M. retusa, Gray, in Griffith's Cuvier [not of Raffin].

7 Sylhet [typical]; ex c. Asiatic Soc. Bengal.

Probably better classed in the next subgenus; I retain, however, M. Brot's classification for the present.

\section{Sub-Genus PARAIIELANIA, Smith.}

P. Zool. S. 1881, type M. nassa, Woodward.

Mr. C. A. White (in 'Nature', 1881, Vol. XXV, p. 101) considers the group identical with that of Pyrgulifera humerosa of Meek (U. S. Geol. Survey, Vol. IV, by Clarence King), a Mesozoic fossil.

\section{Melania [Paramelania] nassa.}

Melania nassa, Woodward, Ann. and Mag.

1 Lake Tanganyika, Central Africa; ex Mr. Damou.

\section{Sub-Genus TAREBIA, H. \& A. Adams.}

Gen. Moll. I, p. 304, as subgenus of Vibex, Oken! Sect. = Sermyla, $\Pi$. and A. Adams, l.c., as subgenus of Melanella, Sw.; type fide Lrot, Conch. Cab., 1874, Mel. granitera.

\section{Melania [Tarebia] lateritia.}

Melania lateritia, Lea, P. Zool. S. 1850, Philippines.

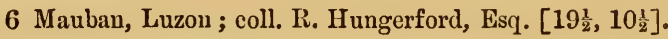

A dark-coloured, short-spired, much-eroded form, without bands.

6 Mindanao; coll. Prof. C. Semper.

Resembling the preceding, but of a yellowish-brown colour and not eroded.

7 Philippines; coll. Baron F. von Richthofen. 
Broadly banded with brown; spire of some specimens short, as in M. Brot's pl. 33, fig. 1B., of others produced as in pl. 33, fig. 15 .

2 Cebu; coll. R. Hungerford, Esq.

74. Melania [Tarebia] procera.

Melania procera, Brot, Conch..Cab. II, 1874, Habit.?, long.36, diam. 14 mil.

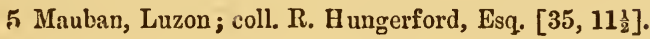

75. Molania [Tarebia] celebensis.

Melania celebensis, Quoy, Toy. Astrol. 1832, Celebes.

1 Moluccas [?] ; cx c. Dr. F. Stoliczka [? ex Landauer].

Labelled "168-Mel. kapii, D. from Moll." [? Moluccas].

76. Melania [Tarebia] riqueti.

Melania riqueti, Grateloup, Act. Soc. Lin. Bord. 1840, p. 433, Bonobay, long. 13, diam.7 mil.; = M. harpula, Philippi, Abb. I, 1845, pl. III, fig. 6, Java ?; Con. Indica, pl. LXYI, fig. 10, Quilon, Cuchin, and Travancore; Sect. Sermyla.

3 Locality ?; ex c. Dr. F. Stoliczka.

Labelled "Bought with Maldive shells."

2 Batavia; ex c. A. Morelet, Esq.

All the above are admirably represented by the above-quoted figure of Philippi ; M. Brot's pl. 34. fig. 6, is mueh less characteristic; the Professor questions the Javanese locality, which, however, would appear to be correct.

\section{Melania [Tarobia] obliquigranosa.}

Melania obliquigranosa, Smith, P. Zool. S. 1878, Formosa-long. 25, diam. 8 mil.

20 Keelung and Tiukang [Formosa]; coll. R. Hungerford, Esq. 


\section{Melania [Tarebia] mauiensis.}

Melania mauiensis, Lea, P. Acad. Philad., 1856, Sandwich Islands.

12 Sandwich Islands; coll. H. Pease and W. Newcomb, Esqrs.

var. subgranifera.

1 "Iles Marianes"; ex c. A. Morelet [as "M, granifera, Lam."]

Rather more distinctly granulose. Long. 23, diam. $8 \frac{3}{4}$ mil.

subvar. ?

2 Halmaheira ; ex Mr. Landauer [as "M. lirata, Bens."].

Can this locality be correct? I can see no difference whatever from the preceding specimen.

\section{Melania [Tarebia] batana.}

Melania batana, Gould, P. Bost. S. I, 1843, Tavoy; Con. Indica, pl. 74, figs. 8-9; fide Brot, belongs to Sect. Melanoides!

8 Tenasserim River; ex c. Asiatic Soc., Bengal.

var. sublineata, nov.

Morelet, Ser. Conch. IV, 1875, p.320 [as n. sp. near M. celebensis and M. granifera], Cochin-China.

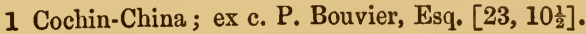

An interesting form with the granular sculpture more or less indistinct, its remarkable nodulose appearance being altogether wanting; this is, however, also in the type form not a permanent character, one or two of my specimens, found with the other Tenasserim ones, approaching the Cochin-China variety very closely in this respect.

10 Penang; ex c. Jritish Museum [as "M. lirata, coll. Cantor"].

\section{Melania [Tarebia] tahitensis.}

Melania tahitensis (Pease), Brot, Conch.-Cab.II. pl.33, fig. 7, p.472, as a

"var. of M. mauiensis," p. 323, l. c.

2 "Navigators' Islands"; coll. Dr. C. E. Tounerre [A. S. B.]. 
Certainly distinct, I think, from $M$. mauiensis; even more closely resembling $M$. lateritia. These specimens agree fairly with the above-quoted figure, only the last whorl is more convexly tumid and the aperture more dilated. Long. 22, diam. $11 \frac{1}{4}$ mil.; anfr. 4.

\section{Melania [Tarebia] rudis.}

Melania rudis, Lea, P. Zool. S. 1850, Amboina; fide Brot, $=$ M. microstoma, Lea, Isl. Negros.

2 Halmaheira ; ex Mr. Landauer.

This is the var. B. of M. Brot [pl. 32, fig. 1A.], which, however, judging from the locality, I consider to be the typical form.

var. ceylonica, nov.

Con. Indica, pl. LXXIV, figs. 7 and 10, and Reeve, fig. 172 [bene].

8 Kalutara, Ceylon; coll. H. Nevill, Esq.

6 Tenasserim Kiver; coll. W. 'Theobald, Esq. [A. S. B.].

These latter agree exactly with the Ceylon specimens. They are the No. 3 ["M. tulerculata"], var. D. of Theobald's Catalogue, page 40 . I should much like to see the locality confirmed. I fear there may have been some confusion.

7 Tenasserim River; ex c, Asiat. Soc. Bengal.

These are doubtless part of the preceding donation, which it was not considered necessary to "mount." Two are of a small, dwarf race, and three are not decollate, in this state resembling very closely the Halmaheira specimens.

\section{Melania [Tarebia] scopulus.}

Melania scopulus, Reeve, Icon., fig. 165, 1860, Locality?; Philippines fide Brot [Cuming].

5 Luzon; coll. R. Hungerford, Esq.

20 Philippines [?]; coll. Baron F. von Richthofen.

These include two or three varieties; one especially with very ventricose last whorl and finer, more close-set sculpture; another is without any longitudinal, decussating sculpture, and is of a uniform dull violet colour. 
var. [? dist. sp.].

2 Cebu ; coll. R. Hungerford, Esq.

A small form, perhaps not adult, near $M$. celebensis, Quoy.

\section{Melania [Tarebia] broti.}

Melania broti (Dohrn), Reeve, Icon. fig. 160, 1860, Ceylon; $=$ M. chocola tum, Brot, Rev. Zool. 1860 ; sp. of Tiaropsis fide Brot.

5 Ceylon; coll. E. L. Layard [A. S. B.] and H. Nevill, Esqrs.

suovar. subrudis.

3 Ceylon; coll. E. L. Layard, Esq. [A. S. B.].

A form not represented by M. Brot ; it may fairly be considered a connecting link between this species and $M$. rudis. Long. 26 , diam. $11 \frac{1}{4}$ mil. ; decollate, $3 \frac{1}{2}$ whorls only remaining. Compare also Mr. Hanley's remarks, Con. Indica, page 32.

\section{Melania [Tarebia] lineata.}

Helix lineata, Gray, Sup. Wood's Index Test. 1828, fig. 68, "East Indies"; = Melania sp., Benson, Gleanings of Science, I, fig. D. and II, page 22 [sine nom.]; = Melania lirata, Benson, J. A. S. B., V, 1836, page 782 [sine descr.].

The synonomy of this species is correctly given by $\mathrm{Mr}$. Hanley in the Con. Indica; M. Brot, on the other hand, has evidently not had the "Gleanings of Science" to refer to, or else he would have found the name of Melania lirata is not mentioned therein.

50 Calcutta ; coll. G. Nevill, J. B. Baxter, and Colonel G. B. Mainwaring.

With one or two undulating, subgranulose keels below the suture only; well represented by the Con. Indica, pl. LXXI, fig. 7.

15 Calcutta; coll. Dr. F. Stoliczka and ex c. Asiatic Soc., Bengal.

Granulose sculpture distinct and well developed; fairly represented by M. Brot's pl. 33, fig. 6, excellently by Reeve's fig. 170 [Sikkim].

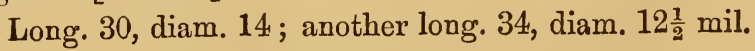

20 Calcutta [in Tanks]; coll. G. Nevill, Esq. 
Long. 19, diam. 8 mil. Granulose sculpture almost obsolete.

6 Teria Ghit; ex c. Dr. F. Stoliczka.

4. Ceylon; ex c. Asiatic Soe., Bengal.

20 Tezpore, Goalundo, Mandalay, Gowhatty, Bhootan; coll. Dr. F. Stoliczka.

var. semigranosa.

Mel. semigranosa, v. d. Busch, Phil. Abb. I, 1844, Java.

Differs conspicuously by the shorter spire, and the more tumidly ventricose last whorl, which is also more produced, \&c. Long. 31, diam. 15 mil.; apex eroded.

15 Java [?] ; coll. Baron F. von Richthofen.

20 Near Moulmein; coll. Baron F. von Richthofen and Dr. F. Stoliczka.

Long. 18, diam. $8 \frac{1}{2}$ mil. May be considered as a connecting link with $M$. batana, Gld.

7 Pegu; coll. Dr. F. Stoliczka.

A fine form with turriculately planulate whorls. Long. 30, diam. 13 mil.

12 Meetan, Tenasserim Province; coll. O. Limborg.

1 Teria Ghat [?]; ex c. Dr. F. Stoliczka.

subvar. pergranosa.

Long. $15 \frac{1}{2}$, diam. $7 \frac{1}{4}$ mil. Granuilose seulpture very prominent.

20 Port Canning; coll. J. B. Baxter, Esq.

var. flavida.

Mel. flavida (Dunker), Fhil. Abb. I, 1844, Hab. ?

6 Java; ex c. A. Morelet, Esq.

9 Calcutta; coll. Dr. F. Stoliczka.

3 Pooree; coll. H. Raban, Esq.

Indian specimens of this variety have the spire more subcylindrically produced than the Javan ones.

2 Teria Ghat; cx c. Dr. F. Stoliczka. 


\section{Sub-Genus TIARA, Bolteu.}

H. and A. Adams, Gen. Moll. I, 1855, as distinct Genus; type restr. Brot, Mel. amarula.

\section{Melania [Tiara] amarula.}

Helix amarula, Linnaus, Syst. Nat. XII, p. 1249, 1\%6\%, "Hab. in Asice fluviis" ; Bruguière, Enc. Méth., pl. 458, fig. 6 ; fide March should be “Mel. melanus (Melas), Mtf.," from Mauritius, \&c. ; juv. $=$ M. moreleti, Desh. [not Reeve], Traité Elém. Conch., Hab.? and of Brot, pl.30, fig.2.2A.; juv. ?=Brot, l.c.,pl. 28, fig.5, B. and C. [as M. pagoda, var.].

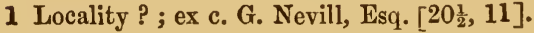

Unfortunately, I have no record concerning this specimen, well represented by M. Brot's pl. 30, fig. 2 [M. moreleti]; the body whorl is smoother and less ventricose, spire more exserted than in young Mauritian specimens.

5 “Trou d'Eau Douce," Mauritius ; coll. G. Nevill, Esq.

A very fine form, near M. Brot's pl. 29, fig. 1A.

5 Mauritius ; coll. G. Nevill, Esq.

The typical form well represented by M. Brot's, pl. 29, fig. 1. Despite M. Brot's valued opinion [1. c., p. 291], I think I may state positively that Deshayes' $M$. moreleti is the young of the typical form, notwithstanding the remarkable " quasi" adult appearance that it invariably presents. These young specimens agree well with pl. 30, fig. 2 A, 1. c.. Dr. Moerch considered a form from the Moluccas as the true Mel. amarula.

subvar. depauperata.

Conch.-Cab. II, pl. 29, fig.1F, Madagascar.

1 Nossi-bé, Madagascar ; coll. J. Caldwell, Esq.

Long. 33, diam. $17 \frac{3}{4}$ mil. Apparently a locally constant race.

subvar. cornuta.

Mel. cornuta, Lea, P. Zool. S. Madagascar.

1 Madagascar; coll. J. Caldwell, Esq. [conf. Conch.-Cab. II, pl. 29, fig. 1A.]. 
var. thiarella.

Melania thiarella, Lamarck, Hist. 1822, "Les Grandes Indes."

1 Mauritius ; coll. Mons. Liénard [A. S. B.].

Agrees with M. Brot's pl. 29, fig. 3.

7 Mauritius; coll. Messrs. J. Caldwell and Liénard [A. S. B.].

Larger; some agree with fig. 1d., some with fig. 3B., and others again are intermediate. This variety Dr. Moerch considered as a distinct species and identified it as the "Strombus coactus" of Meuschen, 1787 [Hab. ?].

\section{Melania [Tiara] cybele.}

Melania cybele, Gould, P. Bost. S. 1847, Fiji, Navigators' Islands, \&c. ; fide Brot, $=$ M. crenularis, Deshayes, Mag. Zool. 1844, Philippines.

I doubt if Mel. diadema, Lea, from the Philippines and Amboyna, should be specifically separated. Dr. Morch considered Mel. crenularis as distinct; he does not mention either Lea's or Gould's species.

3 Fiji ; ex c. G. Nevill, Esq.

Well represented by M. Brot's pl. 29, fig. 2B.

var. amara.

Melania amara, Moerch, J. de C., 1872, Little Nicobar, for Mel. mitra, Reeve [not of Meuschen], Sumatra.

1 Great Nicobar; coll. F. A. de Roepstorff, Esq.

Apparently very local, as I have only seen two specimens from the interior of the island. It is a beautiful form with pink-tinged collumella, and is exactly represented by M. Brot's pl. 29, fig. 2 C. ["M. diadema," Lea]. The writer classes Mel. amara as a synonym of Mel. cybele; doubtless he had not seen an authentic specimen. Reeve's figures 175 b. c. from Sumatra, though larger, are also very close.

\section{Melania [Tiara] winteri.}

Melania winteri (v. d. Busch), Philippi, Abb. I, 1845, Java; fide Brot type of his section Tiaropsis.

8 Mauban; Luzon; coll. R. Hungerford, Esq. 


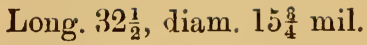

Prof. Brot has omitted to record the Philippine "habitat" of this species, though it is duly mentioned in Reeve. The latter's fig. 157 A well represents the form.

88. Melania [Tiara] setosa.

Melania setosa, Swainson, Quart. Journ. Sci. 1824; apud Brot, from Waigiou, N. Guinea, Sumatra [Point Palmas], Philippines [Samar'] and vars. from Amboyna, Bali and Celebes.

2 Locality ? ; ex c., G. Nevill, Esq.

\section{Melania [Tiara] villosa.}

Melania villosa, Philippi, Zeits. Mal. 1848, Loc. ?;=M. speciosa, A. Adams, P. Z.S. 1853, N. Caledonia.

5 New Caledonia ; ex c. P. Bouvier and J. Caldwell, Esqrs.

Sub-Genus PLOTIA, Bolten.

II. and A. Adams, Gen. Moll. I, 1855, as sub.genus of Tiara; type restr. Brot, Mel. spinulosa.

\section{Melania [Plotia] oualanensis.}

Melania oualanensis (Pease), Tryon, J. Amer. Conch. II, 1866, Ovalan [Caroline Isl.], not M. ovalanensis, Mousson.

4 Ovalan [typical]; coll. H. Pease, Esq.

\section{Melania [Plotia ?] jugicostis.}

Melania jugicostis [Benson], Hanley, Con. Indica, 18\% , pl. 28, fig. 12, Tenasserim River [sine descr.]; Nevill, J. A. S. B., 1S\%7, as of sect. Melanoides [cum descr.].

2 Myadoung [Frontier of Yunan]; coll. Dr. J. Anderson.

3 Qualla Kangsa [Perak]; coll. Ed. 'Towusend, Esq.

\section{Melania [Plotia ?] lamberti}

Melania Lamberti, Crosse, J. de C., 1869, N. Caledonia.

2 N. Caledonia ; ex c. P. Bouvier, Esq. 


\section{Melania [Plotia] datura.}

Melania datura, Dohrn, P. Zool. S. 1858, Ceylon, long. 21, diam. 11 mil.; Con. Indica, pl. 73, fig. 10.

M. Brot unites, as a variety, the forms from Ceylon figured in the Con. Indica, pl. 110 , fig. 7 as $M$. scabra, var. spinulosa and fig. 10, as $M$. acanthica, Dohrn; this is, I think, more than doubtful, as also, I consider, is his statement that the three forms are found together; this is quite opposed to all I know on the subject, and it would be desirable if the Professor would give his authority for the statement.

2 Ceylon; coll. H. Nevill, Esq.

\section{Melania [Plotia] acanthica.}

Melania acanthica, Lea, P. Zool. S. 1850, Manilla and Negros, long. 0.8 diam. 0.4.

var. roepstorffiana, nov.

3 Andamans [type var.] ; coll. F. A. de Roepstorff, Esq.

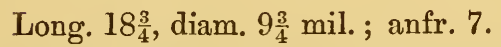

Very near M. Brot's pl. 28, fig. 10A. [Philippines].

subvar. brevispira, nov.

4 Andamans ; coll. F. A. de Roepstorff, Esq. $\left[12 \frac{3}{4}, 6 \frac{3}{4}\right]$.

subvar. subscabra, nov.

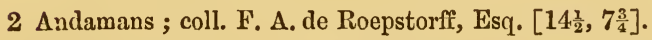

A connecting link with $M e l$. scabra, the spines being rather less developed.

\section{Melania [Plotia] scabra.}

Buccinum scabrum, Mïller, Hist. Verm. 1774, Coromandel $=M$ M. spinulosa, Lamarck, Hist. 1822, Timor, long. 10 lignes; fide Brot, $=M$. doreyiana, Lesson, 1830, Nouv. Guinée, long. 8, diam. 3 lignes; fide Brot= IM. denticulata, Lea, P. Z. S. 1850, I. Negros [not of Reeve ]; var. $=$ M. cochlea, Brot, Conch.-Cab. II, 1874, pl. 27, fig. 10, "India," [as of Lea, P.Z.S. 1850, Hab. ?] ; var.=M. myurus, Brot, Rev. Zool. 1S60, type Juva, also Halmaheira [Landauer] and Borneo [Issel] 
M. Brot, on page 269, correctly quotes "I. Negros " [Philippines] as the original locality of Lea's type of Mel. denticulata; on page 268, however, he says that.M denticulata, Lea, from "Afghanistan," is not separable; but the species figured for $M$. denticulata by Reeve is from the "Philippines," and probably belongs to Mel. pagoda, Lea.

15 Kandy ; coll. E. L. Layard (A. S. B.) and H. Nevill, Esqrs.

Well represented by the Con. Indica, pl. 73, figs. 1-3, and

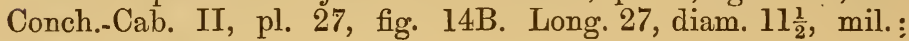
slightly decollate.

10 Paniar River, Cuddalore; coll. H. F. Blanford, Esq. [A. S. B.], and Colonel Beddome.

20 Madras ; ex c. Madras Museum [as "M. collistricta"].

A few of these have the spines prominently developed, as in the Mascarene form, exactly resembling Conch.-Cab. pl. 28, fig. $10 \mathrm{~A}$; most of the specimens, however, approach nearer Con. Indica, fig. 1. Long. 21, diam. 9 mil. [spec. max.].

30 Sibb, Biluchistan; coll. W. T. Blanford, Esq.

A small elegant form, apparently very constant in its characters, fairly intermediate between $M$. scabra and $M$. elegans. Long. $18 \frac{1}{2}$, diam. 8 mil.

28 Madras ; coll. Dr. F. Stoliczka and ex As. Soc., Bengal.

Mostly the ordinary, typical form, but one of Dr. Stoliczka's exactly resembles Con. Indica, pl. 73, fig. 5 [M. elegans].

8 Mauritius ; coll. G. Nevill, Esq., and Dr. F. Stoliczka.

A fine form, with the last whorl abnormally swollen, analogous to $M$. commersoni, Mor. in relationship to $M$. tuberculata; Conch.-Cab, II, pl. 27, fig. 15A [Moluccas], well represents the variety. Long. 29, diam. $13 \frac{1}{2}$ mil.; decollate, five whorls only.

9 Locality ?; ex c. Dr. F. Stoliczka.

There was unfortunately no label with these remarkable specimens; they somewhat resemble the preceding, and are, I suspect, from Mauritius.

50 Calcutta ; coll. Dr. F. Stoliczka and G. Nevill, Hsq. 
Well represented by Con. Indica, pl. 73, fig. 1. Some have the spines moderately developed, as in the Madras specimens already noticed. A single abnormal specimen has the spines remarkably duplicated, in a " frondulose" manner.

13 Balapiti, Ceylon; coll. G. Nevill, Esq.

A pretty, little form with the spines well developed, closely resembling a Mauritian form ; Conch.-Cab. II, pl. 27, fig. 14C, ["Trichinopoly"], pl. 28, fig. 5A. ["M. pagoda"] and l. c., fig, 10A. ["M. acanthica "], long. 12, diam. 9 mil.; strongly decollate, 3 to $3 \frac{1}{2}$ whorls only.

13 Mauritius ; coll. J. Caldwell and G. Nevill, Esqrs.

Like the preceding, but larger ; Reeve's fig. 183 well represents it ["M. scabra"].

4. Cuddalore [Madras Pres.] ; coll. Colonel R. H. Beddome.

6 Karachi and Kathiawar; coll. F. Fedden and J. A. Murray, Esqr's.

In bad condition. I fancy a large series would show they belong to the variety called "Mel. sobria" by M. Brot [pl. 27, fig. 10 l. c.].

5 Umarkote, Sind; coll. Dr. F. Stoliczka.

Resembling the preceding apparently; also in a wretched state.

3 Cochin-China ; ex c. A. Morelet, Esq.

Exactly like the Biluchistan specimens.

1 Barak River, Cachar; coll. J. Wood-Mason, Esq.

20 Matelle, Ceylon; coll. F. Layard, Esq.

Resembling Con. Indica, pl. 73, fig. 1.

3 Myadong, Upper Burma ; coll. Dr. J. Anderson.

Well represented by Reeve's fig. 186 [M. spinulosa from Solomon I.].

8 Teria Ghat, Mandalay, Bombay, Cuddalore, Taptee River; ex c. Dr. F. Stoliczka.

3 Timor; ex c. A. Morelet, Esq.

I suppose these may be considered as the typical form of $M$. spinulosa. They agree closely with the Biluchistan and Cochin-China specimens.

16 Java ; coll. Baron F, rou Richthofen. 
These are the var. nodose-costata of Mousson, Moll. Java, pl. 11, fig. 11.

6 Mahé, Seychelles; coll. G. Nevill, Esq.

Well represented by M. Brot's pl. 7, fig. 14A. [Ceylon], only rather smaller.

4. Mauritius ; coll. G. Nevill, Esq.

Fairly intermediate between var. granum and the form represented by M. Brot's pl: 27, fig. 14. Long. 19, diam. $8 \frac{3}{4}$ mil.

\section{var. elegans.}

Melania elegans, Benson, J. A. S. B. V., 1836, p. 782, for Melania sp. ? Gleanings of Science, II, 1830, p. 22, Rivers Gumti, Belwa and Cen; Hanley, Con. Iudica, pl. $\%$, figs. 5-7, N. Oude and South India, as Melania scabra, var. elegans, Bs.; Brot, Conch.-Cab. II, pl. 28, fig. 9, as "Mel. elegans, Bs."

I consider Mr. Hanley is undoubtedly right in uniting this form to Mel. scabra, the transition from the one to the other being clearly traceable.

10 Ferozepore [Punjab]; coll. Captain R. C. Temple.

Found in a well. This remarkable " habitat" confirms Captain Hutton's record of a similar observation, J. A. S. B., 1834. These specimens are the only ones 1 have seen that agree with M. Brot's above-quoted flgures.

8 "India"; ex c. Asiatic Soc., Rengal.

These are perhaps typical M. elegans, from Benson.

6 Bolan Pass; coll. Captain F. W. Hutton [A. S. B.].

Long. 24, diam. 10 mil. A very solid form, presenting some of the characters of M. Brot's pl. 27, fig. 10 [M. cochlea], but with much shorter spine. This may be Reeve's fig. 178, " $M$. elegans," Benson, MS., from Afghanistan, in which, however, the ribs on the last whorl are less developed.

10 Sunderbunds; ex c. Dr. F. Stoliczka [22, 10].

Closely resembling the preceding; I should like to see the locality confirmed, as I have never found anything like it myself in these parts.

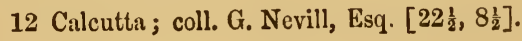


Well represented by the above-quoted figures in the Con. Indica ; it is doubtless Benson's original "elegans."

var. myurus.

Melania myurus, Brot, Rev. Zool. 1860,pl. 16, fig. 3, Java ; Conch.-Cab. II, pl. 28, fiy. 1, Java, Halmaheira [Landauer] and " ?Borneo [Issel]."

I consider this form scarcely separable even as a variety. I have seen equally cylindrical forms from India, \&c.

3 Halmaheira; ex Mr. Landauer [16 $\left.\frac{1}{2}, 7 \frac{1}{2}\right]$.

Intermediate between the two larger figures in the Conch.Cab., with more of the ordinary "scabra" type.

2 Halmaheira; ex Mr. Landauer.

Resembling pl. 28, fig. 1B., l. c.

var. ?

1 Java, Philippines or Siam?; coll. Baron F. von Richthofen.

Long. $26 \frac{1}{2}$, diam. 11 mil. A very striking form, with very tumid and ovately produced last whorl, unlike any figure I can find.

var. granum.

Melania granum (v. d. Busch), Philippi, Abb. I, 1S44, Java; fide Brot, distinct sp. and = Mel. scabrella, Mousson [not of Phil.]; var. bucci. noidea, Mousson, Moll. Java, pl. 12, fig. 4, Lake Tirir.

5 Java; ex c. A. Morelet, Esq.

Four of these are the spineless "var. buccinoides," of Mousson.

12 Mauritius; coll. J. Caldwell and G. Nevill, Esqrs.

Anfr. 3; long. 12, diam. 7 mil. An abundant small form, almost exactly resembling Javanese specimens. The spiral striation is quite as developed. Always strongly decollate. 
96. Melania [Plotia], sp. ?

4. Telagu Patengan [Java]; coll. Baron F. von Richthofen.

Long. $17 \frac{1}{2}$, diam. 7 mil. I am quite unable to identify this species. It somewhat resembles $\mathbf{M e l}$. jugicostis, also Mel. unifasciata.

\section{Melania australis.}

Melania Australis, Lea, P. Zool. S. 1850, Victoria River; Smith, Voy. Erebus, pl. 4, fig. 3.

\section{? var. [? distinct sp.].}

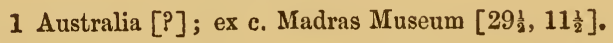

A very doubtful form, with doubtfully correct habitat. Labelled "Mel. australis, Reeve, Australia." I may take this opportunity of warning European conchologists against the labels, identifications, \&c., of the Madras Museum: I have found both equally untrustworthy.

\section{Sub-Genus MAINWARINGIA, nov.}

Type M. paludomoidea, n. sp.

A new generic form, apparently connecting Melania and Paludomus, distinguished by the presence of rows of hairs or bristles, especially noticeable on the body whorl, which is ornamented with broad revolving brown bands; not umbilicate; apex remarkably acute, "quasi styliform" ; peristome acute, columella thickened and excavately inflected. Operculum transparent, of one substance throughout,- that is, without any thickened process for attachment, - of very light horn-colour, without sculpture, ovate, acuminate at one end, at the other rounded, and distinctly terminally subspiral, with $2 \frac{1}{2}$ whorls, closely resembling Adam's pl. XXXV, fig. 10A-B., operculum of Hydrobia ulvo.

\section{Melania [Mainwaringia] paludomoidea, n. sp.}

Long. $9 \frac{1}{2}$, diam. $4 \frac{1}{12}$; apert. long. $3 \frac{3}{4}$ mil.

Inperforate, conically produced, spire turretedly acuminate, apex very acute, suture distinct; of a rather yellowish, bright 
" horn"-colour, girt on the last whorl with three distinct, broad, dark-brown bands, showing clearly within the aperture ; spirally regularly striated, below the slight subangulation of each whorl, the striæ prominent, with a minutely "quasi punctate" appearance, the above becoming somewhat obsolete; covered more or less with rather long and well-developed hairs; whorls nine, the apical three exceedingly minute, white and sculptureless, the next five slightly subangulate a little above the middle, cylindrically turreted, last whorl rather tumidly convex, about the same length as the spire; aperture rather small, vertically ovate, peristome acute, columella thickened, dark violet coloured, inflested obliquely, at base sharply angulate. Operculum as above described; it seems to me slightly convex.

6 Mutlah River at Port Canning; coll. Colonel G. B. Mainwaring, J. Wood-Mason, Esq., and Dr. F. Stoliczka.

Found by Colonel Mainwaring in brackish water on trunks of trees submerged at high tide.

\section{Sub-family PALUDOMIN $\mathcal{E}$, Stoliczka.}

\section{Genus PALUDOMUS, Swainson.}

Tieat. Malac. 1840, $p .340$, types Melania globulosa, conica and retusa, Gray; Layard, Ann. Mag. 185̃5, type restricted Paludomus conica, Gray.

\section{Paludomus conica.}

Melania conica, Gray, Griffith's Cuvier, 1834, and Hanley, Con. Misc. fig. 34 [type]; fide Hanley, Con. Indica, p. $50=$ P. rudis, Reeve, P. Z. S., 1852, Loc.?; = Melania crassa, Busch, Phil. Abb. 1847, Bengal [not Pal. crassa of Issel] ; vars. ? = P. maurus, Rv., P, Z. S., 1852, Ganges, and $P$. paludinoides, $R v$., Sikkim.

7 Gowhatty; coll. Museum Collector.

Resembles M. Brot's pl. II, fig. 14 .

20 Teria Ghat, Mangken Rv., Digong Rv. and Jaintia Hills; coll. Colonel H. H. Godwin-Austen.

Well represented by M. Brot's pl. II, fig. 13.

6 Sylhet; ex c. As. Soc. Bengal. 
Very finc specimens, long. 28, diam. 22 mil., conf. Brot, pl. II, fig. 12.

10 Samagooting; coll. S. E. Peal, Esq.

7 Cachar; coll. Dr. Jerdon [A. S. B.].

12 Assam; ex c. Dr. F. Stoliczka.

30 Silcuri [Cachar]; coll. J. Wood-Mason, Esq.

subvar. cherraensis, nov.

Long. 16, diam. $13 \frac{1}{2}$, mil. A short spired form, closely re sembling $P$. globulosa in shape.

6 Teria Ghat [type subvar.]; coll. Colonel H. H. Godwin-Austen.

2 Mangken River, Assam; coll. Colonel H. H. Godwin-Austen.

subvar. dihiriensis, nov.

Long. $17 \frac{1}{2}$, diam. $12 \mathrm{mil}$.

6 Stream on the Dihiri Hill [Brahmaputra watershed]; coll. Colonel H. H. Godwin-Austen.

subvar. chittagongensis, nov.

Long. 21, diam. 14 mil.

Resembling M. Brot's pl. II, fig. 13, but with the spire even more produced and the last whorl less tumid.

10 Clittagong [type var.]; coll. H. Raban, Esq.

var. sibsaugorensis, nov.

Long. 20, diam. 14 $\frac{3}{4}$ mil. Agrees well with M. Brot's pl. 8, figs. 8-9, "P. paludinoides, Reeve, Himalaya," which is probably this variety and not Reeve's Sikkim form.

8 Sibsagar; coll. S. E. Peal, Esq.

var. pealiana, nov.

Long. 19⿺辶, diam. 15 mil.

5 Assam ; ex c. Asiat. Soc. Bengal [coll, Robiuson, Esq.].

1 Sibsagar; coll. S. E. Peal, Esq. 
var. jaintiaca, nov.

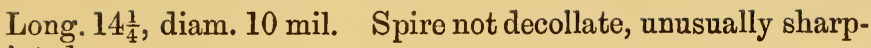
pointed.

4. S. Jaintia Hills; coll. Colonel H. H. Godwin-Austen.

var. kopiliensis, nov.

Long. $24 \frac{1}{2}$, diam. 21 mil. Whorls $1 \frac{1}{2}$ only, the apical ones being decollate, last whorl remarkably inflated, subangulate.

10 Kopili River, North Assam; coll. Colonel H. H. Godwin-Austen.

10 North Assam; ex c. Dr. T. Oldham.

subvar. nana, nov.

Long. 16, diam. $14 \frac{1}{2}$ mil. Only $1 \frac{1}{2}$ whorls.

4 W. Khasi Hills ; coll. Colonel H. H. Godwin-Austen.

var. paludinoides.

Paludomus paludinoides, Reeve, P. Zool. Soc., 1852, Ganges.

This is perhaps a distinct species, but my series is not large enough for me to judge.

2 Sikkim; coll. Captain Sherwill [A. S. B.].

These agree with Con. Indica, pl. 123, fig. 9.

subvar.

2 Sikkim; coll. Captain Sherwill [A. S. B.].

Like the preceding, but spirally sulcate.

subvar.

3 Sikkim; coll. Captain Sherwill [A. S. B.].

2. Paludomus ornata.

Paludomus ornata, Benson, Ann. Mag. 'XVII, 1856, "Regno Burmanico," long. 18, diam. 13 mil.; Con. Indica.pl. 108, fig. 8, and Conch.-Cab. II, pl. \%, figs. 18-20 [operculum bene].

6 Pegu; ex c. Dr. F. Stoliczka.

20 Ava aud Mandalay; coll. Dr. J. Anderson. 


\section{Paludomus andersoniana.}

Paludomus andersoniana, G. Nevill, Journ. Asiat. Soc., Bengal, 187\%, Bharró l. c. $1881, p l . V$, fig. 2 .

30 Bhamô [type], Mandalay, Ava, and Kabyaet; coll. Dr. J. Anderson.

\section{var. myadoungensis.}

P. andersoniana, var. myadoungensis, G. Nevill, J. A. S. B., 1881, Mya. doung.

A small, decollate, and prettily marked variety, with the whorls more rounded.

Long. 15, diam. 12 mil.

2 Myadoung, Upper Burma [type var.] ; coll. Dr. J. Anderson.

subspecies peguensis [an potius sp. dist. ?].

Paludomus andersoniana, var. peguensis, G. Nevill [an sp. n. ?], Journ. Asiat. Soc., Bengal, 187\%, Pegu; Con. Indica, pl. 108. fig. 6, as "P. regulata, var."; Brot, Conch.-Cab. II, pl. \%, figs. 2.3, as " $P$. under. sonianus, var."

20 Pegu Yoma [type var.] ; coll. Dr. F. Stoliczka, \&c.

3 Ava [?]; ex c. Dr. F. Stoliczka.

subvar. nana.

G. Nevill, J. A. S. B., 1ฐ81, Pegu.

Long. 15, diam. 11 mil. Spiral sculpture a trifle less distinct.

12 Pegu; ex c. Dr. F. Stoliczka.

\section{Paludomus regulata.}

Paludomus regulata, Benson, Ann. Mag. XVII, 1856, Thyet-Myo, long. 19 to 24, diam. 12 to 14 mil.; Con. Indica, pl. 108, fig. 5; Brot, Conch. Cab, II, pl. \%, figs. 14 and 17 [not 15].

5 Thyet Myo; ex c. Asiat. Soc., Bengal [coll. W. Theobald, Esq.]. 20 Prome; coll. W. T. Blanford. Esq.

The largest I have seen measures long. 24, diam. 17 mil. 
subvar. minor.

Brot, Conch.-Cab. II, pl. 7, fig. 16.

8 Burma; ex c. Dr. F. Stoliczka.

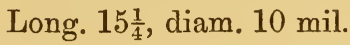

\section{Paludomus blanfordiana.}

Paludomus blanfordiana, G. Nevill, J. As. Soc., Bengal, 1877, Ava, \&c., and l.c. 1881, pl. V, fig. 3 ; Con. Indica. pl.108, fig. 9, as "P. labiaia, from Tongoop" [not P. labiata, Benson, from Tenasserim].

12 Ava [type] and Bassein District [Arakan]; coll. Dr. J. Anderson, \&c. 25 Gowhatty [Assam]; coll. Museum Collector.

\section{Paludomus petrosa.}

Paludin petrosa, Gould, P. Bost. S. 1843, Tavoy; = Paludomus labiosa, Benson, Aun. Mag. 1856, Tenasserim Talley, long. 13, diam. 11 mil.; [not P. labiosa, Con. Indica, pl. 108, fig. 9, from Tongoop]; Nevill, J. A. S. B., 1881, pl. V, fig. 5, Tavoy.

7 Tavoy [typical]; coll. W. Theobald, Esq. [A. S. B.].

Gould's original description I consider excellent, though unfortunately he gives no measurements; at any rate it is quite unmistakeable; Benson probably overlooked it, owing to the species having been described as a Paludina.

\section{Paludomus burmanica.}

Paludomus burmanica, G. Nevill, J. Asiat. Soc. Beagal, 1877, Yaylaymav,

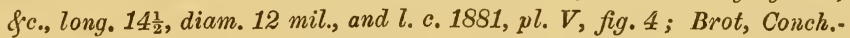
Cab. II, pl. 8, fig. 12 [sp. juv.].

8 Mandalay [not quite adult]; coll. Dr. J. Anderson.

7 Yaylaymaw [type]; coll. Dr. J. Anderson.

\section{Paludomus species?}

3 Java? [not quite adult]; coll. Baron von Richthofen.

\section{Paludomus reticulata.}

Paludomus reticulata, W.T. Blanford, J. As. Soc., Bengal, 1870, N. Cachar, alt. 19, diam. 17 mil.; Con. Indica, pl. 108, fig. 4.

6 Mangken River, N. Jaintia [typical]; coll. Coloncl H. H. GodwinAusten. 


\section{Paludomus isseli.}

Paludomus isseli, Brot, Conch.-Cab. II, 1880, for P. crassus, Issel, Ann。 Mus. Civ. Genova, 1874, p. 45s, Sarawak [not P. crassus, Busch].

3 Sarawak [typical]; ex c. Prof. A. Issel.

Can be easily distinguished from $P$. conica $[=$ crass $\alpha$, Busch $]$ by its smaller size, absence of any trace of bands within the aperture, and, especially, by there being no trace of the impressed spiral sulcations near the suture so characteristic of $P$. conica.

Long. 18, diam. $13 \frac{1}{2}$ mil.

\section{Paludomus lutea.}

Paludomus lutea, H. Adams, P. Zool. S., 1874, Borneo;=P. moreleti, Issel, Ann. Mus. Civ. Genova, 1874, Borneo, long. 15, diam. 9 mil.

1 Sarawak [typical]; ex c. Prof. A. Issel.

\section{Paludomus borneensis, n. sp.}

Issel, Ann. Mus. Civ. Genova, 1874, Sadong, as Paludina hamiltoni, Metcalfe, var persolida, nov.; Martens, Zool. Rec. XII, 18\%5, p. 164.

2 Sadong, Sarawak [type]; ex c. Prof. A. Issel.

\section{Paludomus obesa.}

Melania obesa, Philippi, Abb. 1847, pl. 4, fig. 3 [bene] Hab.?;=P. [Rivulina] maculatus, Lea, Proc. Philad. Ac. 1856, Ahmednuggur and Obs. XI,,$l .22$, fig. 10; ?= P. parva, Layard, Ann. Mag. 1855, Bombay [not of Brot, or Con. Indica].

20 Bombay Pres.; coll Rev. S. B. Fairhank, \&c.

18 Khandala; coll. Rev. S. B. Fairbank.

20 Rajghur Hill, Decean; coll. W. T. Blanford, Esq.

\section{Paludomus chilinoides.}

Paludomus chilinoides, Reeve, Icon.1847, fig. \%, Ceylon; Conch-Cab. II, pl. 6 , figs. $8-9$.

6 Ballepane, Ceylon; coll, H. F. Blanford, Esq. [A. S. B.].

5 Ceylon ; coll. E. L. Layard and Dr. Kelaart [A. S. B.].

20 Ceylon; coll. H. F. Blanford and H. Nevill, Esqrs.

4 Balcadua Pass, Ceylon; ex c. As, Soca, Bengal. 
var. [? dist. sp.].

1 Hangwelle Ella, Badulla; coll. F. Layard, Esq.

Probably quite a distinct species.

var. lævis.

Paludomus lævis, Layard, Ann. Mag. 1855, long. 11, diam. 7 lines, "same Locality as P. chilinoides"; Conch.-Cab. II, pl.7, fig. 1 [bene], not Con. Indica, pl. 108, fig. 3.

7 Ceylon ; coll. H. Nevill, Esq. [sent as " P. lavis, Lay.' ].

A very distinct and well-marked form, perhaps specifically separable. Much more divergent from typical specimens than any of the forms so weli represented by Mr. H. F. Blanford, l. c. It is a quite distinct form from that figured in the Con. Indica, as recorded further on.

\section{Paludomus fulgurata.}

Paludomus fulguratus, Dohrn, P. Zool, S. 1857, Ceylon; Con. Indica, pl. 123, fig. 1; P. chilinoides, var., Brot, Conch.-Cab. II, pl. 6, fig. 11 ; ?=P. phasianinus, Reeve, P. Z. S. 1852, "Seychelles."

1 Ceylon; coll. H. Nevill, Esq. [labelled "very rare "].

Quite distinct from the preceding species, as well shown by the figures iu Con. Indica. Hanley's fig. 62, Conch. Misc., as $P$. phasianinus, $R_{v}$., is almost certainly the same sp. There can, in my opinion, ke no doubt of Reeve's error as to the locality ; it most certainly never came from the Seychelles.

\section{I6. Paludomus constricta.}

Paludomus constrictus, Reeve, Proc. Zool. Soc. 1852, Ceylon, "coll. Layard"; Con. Indica, pl.126, fig. 1 ; P. chilinvides var., Brot, Conch.-Cab. II, pl. 6, fig. 15 .

4 Ceylon; coll. H. Nevill, Esq. [sent as "P. tumida, nov."].

6 Ceylon; ex c. Dr. F. Stoliczka [coll. H. Nevill, Esq.].

The above-quoted fig. in the Conch.-Cab. is, as noted by M. Brot, not characteristic. The last whorl is as often ornamented with rows of small dots as it is without them; the painting is, however, of a different character from that of $P$. chilinoides. 
Mr. Layard, in the Ann. Mag. for 1855, describes the operculum as " typical, but apex much inclined to the left."

\section{Paludomus tanjoriensis [emend.].}

Helix tanschaurica, Gmelin, Syst.1790`; Chemnitz, Conch.-Cab. I, Vol. IX, 1786, as Helix tanschauriensis of Gmelin; emend. H. F. Blanford, Trans. Lin. Soc. 1863.

5 Poonamalee, Madras ; ex c. Asiatic Soc., Bengal.

30 Madras ; coll. H. F. Blanford, Esq.

20 Trichinopoly ; coll. H. F. Blanford, Esq.

30 Courtalier Rv.; ex c. Madras Museum.

var. palustris.

Paludomus palustris, Layard, Ann. Mag. 1855, Ceylon.

1 Ceylon; ex c. Dr. F. Stoliczka.

7 Ceylon; coll. E. L. Layard, Esq. [A. S. B.].

13 Ceylon; coll. H. Nevill, Esq.

I unhesitatingly agree with Mr. Blanford, in opposition to the decided opinion of M. Brot, in uniting the Madras and Ceylon forms ; the greater relative shortness of the last whorl is the most marked character, the peculiar sculpture, as shown in the Con. Indica, pl. 126, figs. $2-3$, is seen in young specimens of both. The keels of the apical whorls are difficult to detect in the Ceylon form, specimens being always more or less decollate; nevertheless they can be traced every now and then.

var. kadapaensis, nov. [dist. sp. ?].

3 Kadapa District, Madras; coll. W. King, Esq.

Apparently without sculpture; apex not acute ; spire pointed, of four to five whorls, longitudinally, regularly, handsomely flamed, somewhat as in $P$. parva, Layard, but in a less "zigzag"

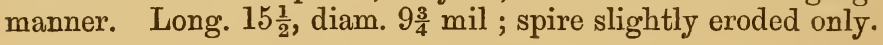

\section{var. malabarica, nov.}

9 Travancore ; coll. Colonel R. H. Beddome.

5 Puluey Hills; coll. Rev. S. B. Fairbank.

Decollate and in shape exactly resembling the Ceylon var. palustris ; decussating striæ, however, obsolete in the adult, as in the typical Madras form, spiral striation very variable 
in amount of development. Long, $16 \frac{1}{2}$, diam. $11 \frac{1}{2}$ mil.; decollate, three whorls only remaining.

\section{Paludomus monile.}

Paludomus monile [Thorpe], Hanley, Con. Indica, pl. 108, fig. 10, S. India; fide Brot $=$ P. obesa, Phil., var.

3 Udamanchola, Travancore ; coll. Colonel R. H. Beddome $\left[14 \frac{2}{2}, 11\right]$.

3 Travancore ; coll. Colonel R. H. Beddome.

1 Trevandrum ; ex c. W. Theobald, Esq. [coll. Bourdillon, Esq.].

An interesting form intermediate between my preceding var. kadapaensis and the figure (1. c.) of $P$. monile. This species is evidently quite unknown to M. Brot, except, of course, from the original figure, which is not good, being far too brightly coloured, \&c.

1 Balarangam [spec. juv.] ; coll. Colonel R. H. Beddome.

Unfortunately only a single, not adult, specimen, apparently of typical P. monile.

6 South Travancore; coll. Colonel R. H. Beddome.

\section{Paludomus rotunda.}

Paludomus rotunda, W. T. Blanford, J. As. S. Beng. 1870, Truvancore, alt15, diam. 14 mil. ; Con. Indica, pl. 108, fig. 2.

10 Balarangam, Anamallays [typical] ; coll. Colonel R. H. Beddome.

var. microstoma, nov.

Long. 11, diam. 10 mil. Much eroded, $1 \frac{1}{2}$ whorls only remaining.

6 Anamallay Rivers; coll. Colonel R. H. Beddome.

7 Madura Hills ; coll. Colonel R. H. Beddome.

\section{Paludomus inflata.}

Paludomus inflatus, Brot, Conch.-Cab. II, 1879, pl. 8, figs. 25-26, Travanscore and Amerghat, long. 18, diam. 14 mil.

3 Travancore; coll. Colonel R. H. Beddome.

3 Tinnevelly; coll. Colonel R. H. Beddome.

Much finer and more characteristic than the type specimens. Long. 23, diam. 19 mil. 
21. Paludomus, sp.

Con. Indica, pl. 109, fig. 3, as Pal. lavis [not of Layard].

2 Ceylon; ex c. G. Nevill, Esq.

Quite distinct from $P$. chilinoides, may prove to be a var. of the preceding species, or even possibly of $P$.tanjoriensis. There can be no doubt as to the error of identification in the Con. Indica; the last whorl is more or less ornamented with flames, and the sculpture is distinct and peculiar; all of these characters the above recorded two specimens possess; finally, it is not in the least like $P$. chilinoides. Layard distinctly states his $P$. loevis is smooth and without ornamentation on the last whorl; he further considers it donbtfully distinctly from $P$. chilinoides; in all of these respects the shell sent to me by my brother as $P$. lovvis, Lay., agrees perfectly; this latter form is figured neither by H. F. Blanford, nor in the Con. Indica.

\section{Paludomus ajanensis.}

Paludomus ajanensis, Morelet, Ser. Conch. II, 1860, Hafoun near Cape

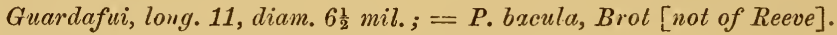

15 Mahé I., Seychelles; coll. G. Nevill, Esq.

4 Loc.? ; ex c. Dr. F. Stoliczka.

The measurements agree exactly with those of M. Morelet; the spire is rather more acuminate, and the whorls less convex, than in original figure.

\section{var. silhouettensis, nov.}

Paludomus, n. sp. ?, Nevill, P. Zool. S. 1869, Silhouette.

Under the lens, beautifully and regularly, very closely, spirally striate, striæ not so coarse as in the preceding, nor so flexuous, but closer together ; spire shorter, decollate, in the adult specimen of two whorls only, in the one not quite adult of three; texture less solid, epidermis of a brighter green ; whorls more convex, the last one more globosely tumid; aperture larger in proportion and more everted. Adult type of variety-long. 9, diam. $6 \frac{1}{2}$ mil. ; operculum resembles that of typical specimens.

2 Silbouette I., Seychelles [type var.]; coll. G. Nevill, Esq.

\section{Paludomus striatula.}

Paludomus striatula, H. Nevill, MS.

2 Ceylon [type] ; coll. H. Nevill, Esq. [sent as " P. striata, nov.]. 
Long. 18, diam. $13 \frac{1}{2}$ mil.; strongly decollate, two whorls only remaining.

M. Brot's pl. 7, figs. 7.8 in the Conch.-Cab. P. isseli, from Borneo, gives an exact representation of this new and rare Ceylon form ; the well-developed, crowded, almost granulose spiral striation will at once distinguish it.

\section{Paludomus africana.}

Paludomus africanus, von MIartens, Monats. Berlin, 18\%8, p. 29\%, Finboni, coast of Zanzibar.

2 Somali Land [typical]; ex c. Dr. H. Dohrn.

Prof. von Martens has also described a Paludomus ewarata, 1. c., from the same highly interesting locality. Mr. Smith in P. Z. S., 1881, p. 294, seems to consider the form as not separable from Melania ferruginea, Lea, P. Z. S., 1850, Zanzibar $=M e l$. zanquebarica, Petit, J. de C., 1852.

\section{Paludomus stephanus.}

Melania stephanus, Benson, J. A. S. B., 1S36, N. E. Bengal ; Con. Indica, pl. 122, fig. 10; = Melania coronata [v.d. Busch], Phil. Abb. 1S12, Bengal.

11 Sylhet [types]; ex c. As. Soc. Bengal.

20 Khasi Hills; coll. Colonel H. H. Godwin-Austen.

Grows to a large size-long. $28 \frac{1}{2}$, diam. $22 \mathrm{mil}$.

\section{Paludomus travancorica.}

Paludomus travancorica, Beddome ms., Blanford, Journ. Asiat. Soc. Bergal, 1881, Travaneore.

4 Travancore [typical]; coll. Colonel R. H. Beddome.

\section{Paludomus microsculpta, n. sp.}

2 Ceylon; coll. H. Nevill, Esq. [sent as " P. striata, nov., very rare"].

Sub-Genus PHILOPOTAMIS, Layard.

Ann. Mag. 185̃5, type Pal. sulcata, Reeve.

Mr. Layard states, l. c., that the section inhabits mountain torrents, and describes the operculum as possessing a terminal nucleus. 


\section{Paludomus [Philopotamis] globulosa.}

Melania globulosa, Gray, Grifith's Cuvier, 1834, var.=P. bicinctus, Rv. fide Auct.

20 Ambegammoa, \&c., Ceylon; coll. H. F. Blanford and H. Nevill, Esqrs.

Specimens, even in youth, very rarely show such bands within the aperture as are depicted in Con. Iconica, pl. 123, fig. 5.

\section{Paludomus [Philopotamis] bicincta.}

Paludomus bicinctus, Reeve, Proc. Zool. Soc. 1852, "Mountain streams in Ceyion"; Hanley, Conch. Misc. 1854, fig. 42 [type]; a true Paludomus, fide Layard, Ann. Mag. 1855 ; fide Brot=P. abbreviatus, Reeve, Proc. Zool. Soc. 1852, Ceylon.

5 Ceylon ; ex c. Asiatic Soc. Bengal [coll. E. L. Layard, Esq.].

These agree with Mr. Hanley's figure, 1. c., and M. Brot's pl. 5, fig. 11. They measure-long. 20, diam. $15 \frac{1}{2} \mathrm{mil}$.

15 Balcadua Pass, \&ce., Ceylon; coll. H. Nevill and H. F. Blanford, Esqrs.

A smaller form than the preceding, agreeing with Con. Indica, pl. 123, fig. 10, and with M. Brot, pl. 5, figs. 6-7.

4. Ceylon; coll. E. L. Layard, Esq. [A. S. B.].

A form with contracted aperture.

30. Paludomus [Philopotamis] rapæformis.

Paludomus rapæformis, Brot, Conch.-Cab. II, 1880, pl. 5, fig. 10, Locality?, "ex coll. Angas."

2 Matelle, Ceylon; coll. F. Layard, Esq. [as "P. bicinctus" var.].

A very distinct species, admirably described by $\mathrm{M}$. Brot, and which I had long intended describing myself. I have little doubt M. Brot's specimens were from the same source as mine, Mr. Angas having been in correspondence with the late Mr. F. Layard.

\section{Paludomus [Philopotamis] sulcata.}

Paludomus sulcatus, Reeve, Icon. 1847, Ratnapoora, Ceylon.

12 Avisavella, \&c., Ceylon; coll. H. F. Blanford and H. Nevill, Esqrs. 
subvar. minor.

Conf. Brot, pl.5, fig. 18, Ceylon.

6 Ceylon; ex c. Dr. F. Stoliczka.

var. coniracta, nov.

Brot, Conch..Cab. II, pl. 5, figs. 19-20, as "P. sulcatus, var.," from Peradenia.

6 Ambegammoa, Ceylon; coll. H. F. Blanford, Esq.

var. compacta.

"Philopotamis compacta, n. sp.", H. Nevill, in litt. as " almost extinct."

1 Ceylon; coll. H. Nevill, Esq.

Remarkable for its small aperture. Long. $16 \frac{1}{2}$, diam. $11 \frac{3}{4}$ mil.

32. Paludomus [Philopotamis] regalis.

Paludomus regalis, Layard, Ann. and Mag.1855, Ceylon.

10 Ceylon; coll. H. F. Blanford and H. Nevill, Esqrs.

\section{Paludomus [Philopotamis] nigricans.}

Paludomus nigricans, Reeve, Icon. 1847, Ceylon, at 6,000 ft.-" anfr. lavibus subangulatis."

14. Newera Ellia, Ceylon; coll. H. Nevill and F. Layard, Esqrs.

My brother sent to Dr. Stoliczka this, the typical form, as " Phil.misconceptus," nov., evidently considering it distinct from the following, in which view he may prove to be correct.

var. subgranulosa, nov. [? dist. sp.].

Brot, Conch.-Cab. II, 18s0, pl. 6, fig. 6, Newerta Ellia, as "var. B."

12 Ceylon; coll. H. F. Blanford, Esq.

\section{Paludomus [Philopotamis] ventricosula.}

Paludomus ventricosula, H. Nevill, Esq., Ms., Brot, Conch.-Cab. II, 1880, pl.5, fig. 3, as Philopotamis violacea, Layard.

Both shell and operculum admirably figured as above by M. Brot; it is, as there stated, a true Philopotamis, whereas 
P. violacea is a true Tanalia. The latter is not in Mr. H. F. Blanford's collection, and is apparently unknown to Messrs. Brot and Hanley.

2 Ceylon [with opercula]; coll. H. Nevill, Esq.

2 "Kandapanue Ella, 4 Corles," Ceylon; coll. F. Layard, Esq.

Sent to me as Paludomus, n. sp.

2 Hackgalle, Ceylon [with opercula]; coll. H.F. Blanford, Esq. [labelled "T. violacea"].

35. Paludomus [Philopotamis] erronea, nov.

Con. Indica, pl. 108, fig. 7, as P. parva [not of Layard]; ? P. phasianinus, Layard, Ann. Mag. 1S5̃̃, Ceylon [not of Reeve].

6 Hackgalle, Ceylon [at 5,500 ft.]; ex c. As. Soc. Bengal [coll. E. L. Layard, Esq.].

20 Ceylon; coll. H. F. Blanford, Esq.

This is most certainly not the P. parva, of Layard-long. 6 diam. 4 lines-from Bombay "with fine, spiral, brown lines," which is most likely a var. of $P$. obesa, Philippi.

The only ally, I know, of Paludomus erronea is P. nigricans, of which Mr. H. F. Blanford considers it a smooth variety; the above-quoted figure, however, shows the differences at a glance. The operculum is that of Philopotamis. Long. $10 \frac{1}{2}$, diam. $6 \frac{1}{2}$ mil.

Apparently it is not figured by M. Brot; the nearest seems to be his pl. 6, fig. 14, "P. chilinoides, var. phasianinus."

\section{Paludomus [Philopotamis] subdentata.}

Paludomus subdentata, H. Nevill, MS.

Resembles Brot's pl. II, fig. 2 [P. nigricans].

Almost smooth, prettily longitudinally striped, "flames" only slightly flexuous; columella very broadly excavated, alabaster white, subdentate at base; spire truncate, two whorls only remaining, the last one convexly swollen; flames are discernible within the aperture.

Long. $13 \frac{3}{4}$, diam. $9 \frac{3}{4}$ mil.

2 Ceylon [type]; coll. H. Nevill, Esq. 


\section{Paludomus [Philopotamis] decussata.}

Paludomus decussatus, Reeve, P. Zool. S. 1852, Ceylon, "coll. Layard"; Philopotamis sp., H. F. Blanford [not Brot].

1 Ceyion [with opere.]; coll. H. Nevill, Esq., [as "very rare"].

3 John's Hill, Ceylon; coll. H. F. Blanford, Esq.

The characteristic sculpture is very distinct in my brother's fine specimen.

Sub-Genus TANALIA, Gray.

Proc. Zool. Soc., 1854,type Nerita aculeata, Chemnitz; sect. = Stomatodon, Benson, Ann. Mag. 1862, type Pal. stomatodon [dist. Genus, fide Brot, 1950]. rents.

Mr. Layard states that the section inhabits mountain tor-

Dr. Stoliczka described and figured a tertiary species from the N. E. Alps as T. pichleri, Hörnes, in the Sitz. K. Ak. Wien XXXVIII, 1859; I think this form is correctly classified, but probably his figs. 7-9 represent a distinct species from fig. 6 .

\section{Paludomus [Tanalia] aculeata.}

Nerita aculeata, Chemnitz, Conch.-Cab. fig. 1642, 1786 [not Nerita aculeata, Gmelin = Neritina sp.] ; fide Brot, 1880, should be Tanalia loricata, Reeve, Icon. 1847, Rapids of Adam's Peak, Ceylon; fide Brot, var. = Tanalia similis, Layard.

30 Ceylon; coll. H. F. Blanford and H. Nevill, Esqrs.

Long. 40, diam. $35 \mathrm{mil}$.

subvar. erinacea.

Paludomus erinaceus, Reeve, Proc. Zool. Soc. 1852, Ceylon; $=P$. skinneri, Dohrn, P. Zool. Soc. 1857, Ceylon.

7 Ceylon; coll. H. Nevill, Esq.

I cannot separate $P$. slimneri, Dohrn, even as a sub-variety. var. undata.

Paludomus undatus, Reeve, Icon. 1847, "Adam's Peak, Ceylon" [ fide Brot, not adult].

15 Ceylon; coll. H. Nevill and H. F. Blanford, Fsqrs. 


\section{var. nodulosa.}

Paludomus nodulosus, Dohrn, P. Zool. \$. 185\%, Ceylon.

3 Ratnapoora; coll. H. Nevill, Esq. [as "P.funiculata, Rv."].

subvar. aerea.

Paludomus aereus, Reeve, P. Zool. S. 1852, Ceylon.

The following agree fairly with M. Brot's figs., but not with that of the Con. Indica.

6 Ceylon; ex c. Dr. F. Stoliczka.

var. reevei.

Tanalia reevei, Layard, Ann. Mag. 1855, Ceylon, Ratnapoora.

1 Ceylon; ex c. Dr. F. Stoliczka.

2 Ceylon; coll. H. Nevill, Esq.

Resemble Con. Indica, pl. 124, fig. 5.

subvar. minor.

5 Ceylon; coll. H. F. Blanford, Esq.

Resemble Conch.-Cab. II, pl. 3, fig. 10.

var. layardi.

Paludomus layardi, Reeve, P. Zool. S. 1852, Ceylon.

1 Ceylon ; coll. H. Nevill, Esq. [as " $P$. nodulosa, Dohrn"].

Long. $27 \frac{1}{2}$, diam. $24 \frac{1}{2}$ mil.

The interior markings are "arrow-headed" and very beautiful. It is unlike both Brot's and Hanley's figures,-nearer the latter.

var. funiculata.

Paludomus funiculatus, Reeve, Icon. 1847, Ratnapoora in Ceylon; $?=P$. picta, of Brot, pl.4, figs. 2 and 3 [not of Layard].

14 Ceylon; coll. H. F. Blanford and H. Nevill, Esqrs. 
var. picta.

Paludomus pictus, Reeve, Icon. 185\%, Ceylon, Ratnapoora.

4 Ceylon; ex c. Asiat. Soc., Bengal [coll. E. L. Layard,'Esq.].

4 Yatteantotte, Ceylon; coll. H. F. Blanford, Esq.

subvar. distinguenda.

Paludomus distinguendus, Dohrn, P. Zool. Soc. 1857, Ceylon.

8 Kitoolgalle, \&c., Ceylon; coll. H. F. Blanford and H. Nevill, Esqrs.

These last resemble M. Brot's pl. 4, fig. 4, " $P$. distinguendu, Dohrn," not the $P$. distinguenila of Con. Indica.

subvar. [?]

1 Ceylon; ex c. G. Nevill, Esq.

subvar. torrenticola.

Paludomus torrenticola, Dohrn, P, Zool. S. 1S5s, Ceylon.

3 Ceylon; coll. H. Nevill, Esq. [as " $P$. distinguendus, rare"].

Agree only fairly with Messrs. Brot's and Hanley's figures,a rather smaller form.

\section{Paludomus [Tanalia] neritoides.}

Paludomus neritoides, Reeve, Icon. 1847, Ambegamoa, Ceylon.

12 Pusilawe, Ballepane, \&c., Ceylon; coll. H. F. Blanford and H. Nevill, Esqrs.

4 Ceylon; coll. H. Nevill, Esq. [as " $T$. picta"].

subvar. dilatata [?]

Paludomus dilatatus, Reeve, P. Zool. S. 1852, Ceylon.

1 Ceylon; coll. H. Nevill, Esq.

var. gardneri.

Paludomus gardneri, Reeve, Icon. 1847, "Foot of Adam's Peak."

2 Ceylon; ex c. G. Nevill, Esq. 
var. thwaitesi [?]

Palndomus thwaitesi, Layard, P. Zool. S. 1854, Ceylon.

1 Ceylon; ex c. Dr. F. Stoliczka.

var. tennenti.

Palndomus tennentii, Reeve, Icon. 1847, "Adam's Pealc."

13 Ballepane, \&c., Ceylon; coll. H. F. Blanford and H. Nevill, Escrss

var. globosa.

P. neritoides, var. globosa, Brot, Conch.-Cab. II, 1880, pl. S, fig. 1.

2 Ceylon; ex c. G. Nevill, Esq.

var. dromedarius.

Paludomus dromedarius, Dohrn, Proc. Zool. Soc. 1857, Ceyton.

2 Ceylon; ex c. Madras Museum.

subvar.

P. picta, var. pl. 4, fig. 3 , is perhaps this form; in any case it is a very distinct and curious variety.

2 Ceylon; coll. H. Nevill, Esq. [28, 23].

\section{Paludomus [Tanalia] solida.}

Paludomus solidus, Dohrn, P. Zool. Soc. 185\%, Ceylan.

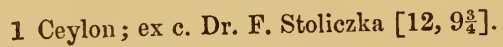

Agrees exactly with M. Brot's pl. 4, fig. 7b. It is probably one of Mr. H. F. Blanford's specimens from Hodlangkanda, Kirime.

\section{Paludomus [Tanalia] hanleyi.}

Paludomus hanleyi, Dohrn, P. Zool. S. 1858, Ceylon, long. 18, diam. 13 mil., Con. Indica, 125, fig. 10.

7 Elletotta Ella and Kaloopaliana, Uluawah, Ceylon; coll. F. Layard, Esq. 
Agree exactly with the figure in Con. Indica, and with original description and measurements.

var. subpicta.

2 Yatteantotte, Ceylon; coll. H F. Blanford, Esq., ex c. As. Soc., Bengal.

var. major [? dist. sp.].

Long. 23, diam. 20 mil.

4. Ceylon; coll. H. Nevill, Esq. [as " $P$. ? Hanleyi"].

The preceding two forms do not agree well together, nor with any figure in the Conch.-Cab. or Con. Indica. The very broad and excavate columella, faintly stained with light brown, is a good characteristic of all the group.

\section{Paludomus [Tanalia] stomatodon.}

Paludomus stomatodon, Benson, Ann. Mag. 1862, Travancore; sect. Stomatodon.

20 Travancore, at 1,000 to 5,000 ft. ; coll. Rev. S. B. Fairbank.

43. Paludomus [Tanalia ?], n. sp.

1 Khasi Hills; coll. Colonel H. H. Godwin-Austen.

Long. 18, diam. 15 mil.

Unfortunately, does not possess its operculum. There is nothing like it in Mr. H. F. Blanford's collection, nor can I find any figure at all approaching it.

\section{Paludomus [Tanalia] violacea.}

Paludomus violaceus, Layard, Ann. Mag. 1855, long. 6, diam. 5 lines; "between Gillymalle and Pallabaddoola, near Adam's Peak;" Con. Inilica, pl. 122, fig. 1; not Philopotamis violacea, Brot $[=$ Phil. ventricosula Nev.].

3 Ceylon; coll. H. Nevill, Esq. [as " $P$. violacea"].

The opercula are those of a true Tanalia; I do not think there can be any doubt whatever that this is the true $T$. violacea. The specimens were collected by my brother in the original locality. 
MELANIID庄.

var. similis.

Paludomus similis, Layard, Ann. Mag. 1855, p. 138, near Ratnapoora.

A rather larger, smoother, and lighter-coloured variety, agrecing in all essential characters, those of the columella, \&c.

1 Ceylon; coll. H. Nevill, Esq. [as " $P$. similis, Lay."].

From the original locality, I believe. 



